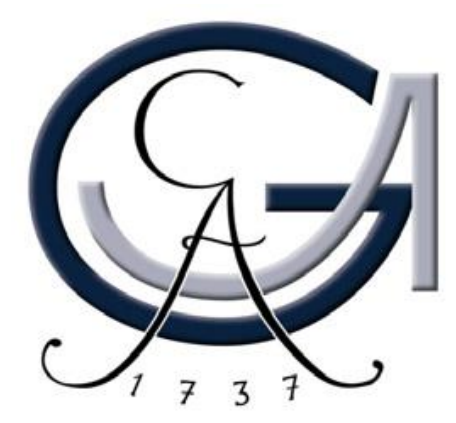

\title{
Organoferrate als Intermediate in Eisen-katalysierten Kreuzkupplungsreaktionen
}

\author{
Dissertation \\ zur Erlangung des mathematisch-naturwissenschaftlichen Doktorgrades \\ "Doctor rerum naturalium" \\ der Georg-August-Universität Göttingen
}

im Promotionsprogramm Chemie der Georg-August University School of Science (GAUSS)

\author{
vorgelegt von \\ Tobias Parchomyk \\ aus Kassel
}

Göttingen, 2018 


\section{Betreuungsausschuss}

(Name, Abteilung/Arbeitsgruppe, Institution)

(Name, Abteilung/Arbeitsgruppe, Institution)

(Name, Abteilung/Arbeitsgruppe, Institution)

\section{Mitglieder der Prüfungskommission}

Referent/in:

(Namen, Abteilung/Arbeitsgruppe, Institution)

Korreferent/in:

(Name, Abteilung/Arbeitsgruppe, Institution)

Weitere Mitglieder der Prüfungskommission:

(Name, Abteilung/Arbeitsgruppe, Institution)

(Name, Abteilung/Arbeitsgruppe, Institution)

(Name, Abteilung/Arbeitsgruppe, Institution)

(Name, Abteilung/Arbeitsgruppe, Institution)

Tag der mündlichen Prüfung: 




\section{Eidesstattliche Erklärung}

Hiermit erkläre ich, Tobias Parchomyk, dass ich die hier vorliegende Doktorarbeit

„Organoferrate als Intermediate in Eisen-katalysierten Kreuzkupplungsreaktionen“

am Institut für Organische und Biomolekulare Chemie der Georg-August-Universität Göttingen unter der Leitung von Prof. Dr. K. Koszinowski im Zeitraum vom 01. Februar 2015 bis zum 26. September 2018 selbstständig angefertigt und keine anderen als die hier angegebenen Quellen verwendet habe.

Göttingen, 26. September 2018

Referent: Prof. Dr. K. Koszinowski

Korreferent: Prof. Dr. F. Meyer 



\section{Danksagung}

Mein Dank gilt Herrn Prof. Dr. K. Koszinowski für die Möglichkeit, diese Arbeit in seiner Forschungsgruppe anzufertigen. Seine offene und freundliche Art der Betreuung sowie seine ständige Bereitschaft, bei Fragen und Problemen beratend zur Seite zu stehen, habe ich in den vergangenen Jahren stets zu schätzen gewusst.

Herrn Prof. F. Meyer danke ich für sein Engagement und seine Bereitschaft, dass Zweitgutachten für diese Arbeit zu übernehmen.

Bei Herrn Dr. S. Demeshko bedanke ich mich für die Durchführung sämtlicher MößbauerExperimente und seine für fachliche Fragen stets offene Bürotür. Den Werkstätten um Dipl.-Ing. V. Meyer danke ich für die Beratung und den Aufbau der im Rahmen dieser Arbeit entwickelten Gasmisch-Apparatur.

Ich danke Andreas, Christoph, Finn, Friederike, Friedrich, Marlene, Rene, Sebastian und Thomas für die fortwährende Hilfsbereitschaft, für fachliche und nichtfachliche Diskussionen und die stets angenehme Arbeitsatmosphäre. Zusätzlich möchte ich Jonathan und allen anderen ehemaligen Bachelor- und Masterstudenten danken, die für eine willkommene Abwechslung im Laboralltag gesorgt haben.

Außerdem bedanke ich mich bei Marlene Kolter und Friedrich Kreyenschmidt für das Korrekturlesen dieser Arbeit.

Mein besonderer Dank gilt meiner Familie, vor allem meinen Eltern und meiner Schwester, die mir während meiner Zeit an der Georg-August-Universität Göttingen eine große, moralische Stütze waren. Ich danke euch für die uneingeschränkte Unterstützung, ohne die diese Arbeit nicht möglich gewesen wäre.

Katja, dir danke ich für all die unvergesslichen Momente und Erlebnisse, die wir miteinander verbracht haben. Ich danke dir vor allem für dein großes Verständnis, dein Mitgefühl und deine unendliche Geduld in Zeiten, in denen der Alltag sehr belastend war. Deine unverwechselbare Art sowie dein offenes und freundliches Wesen konnten immer wieder meine Stimmung heben und bei mir für gute Laune sorgen. Ich freue mich auf unsere gemeinsame Zukunft. 

Teile der im Rahmen dieser Arbeit präsentierten Ergebnisse wurden bereits veröffentlicht in:

Ate-Complexes in Iron-Catalyzed Cross-Coupling Reactions

T. Parchomyk, K. Koszinowski, Chem. Eur. J. 2016, 22, 15609-15613.

Solution and Gas-Phase Reactivity of $\mathrm{Me}_{12} \mathrm{Fe}_{8}{ }^{-}$and Related Cluster lons

T. Parchomyk, K. Koszinowski, Chem. Eur. J. 2017, 23, 3213-3219.

Electronic and Steric Effects on the Reductive Elimination of Anionic Arylferrate(III) Complexes

T. Parchomyk, K. Koszinowski, Chem. Eur. J. 2018, 24, 16342-16347.

Oxidation States, Stability, and Reactivity of Organoferrate Complexes

T. Parchomyk, S. Demeshko, F. Meyer, K. Koszinowski, J. Am. Chem. Soc. 2018, 140, 9709-9720.

Substitution Reactions of Gaseous lons in a Three-Dimensional Quadrupole lon Trap

T. Parchomyk, K. Koszinowski, J. Mass. Spectrom. 2019, 54, 81-87. 



\section{Inhaltsverzeichnis}

1 Eisen-katalysierte Kreuzkupplungsreaktionen .............................................. 1

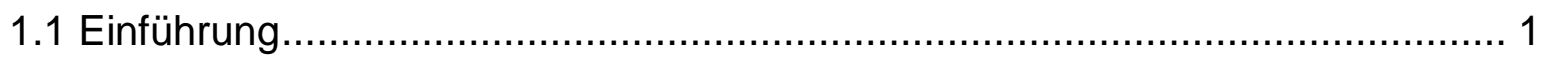

1.2 Synthese: Potential und ungelöste Probleme ……...................................... 3

1.3 Mechanismen: Erkenntnisse und offene Fragen ...................................... 5

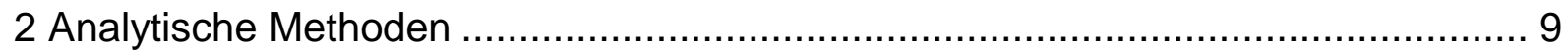

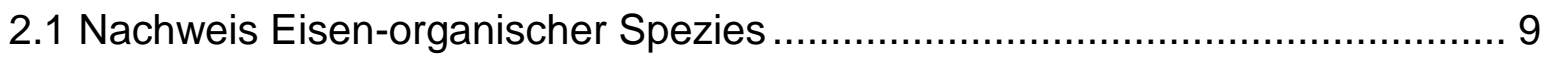

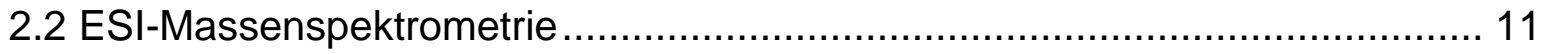

2.2.1 Aufbau eines Massenspektrometers .................................................. 11

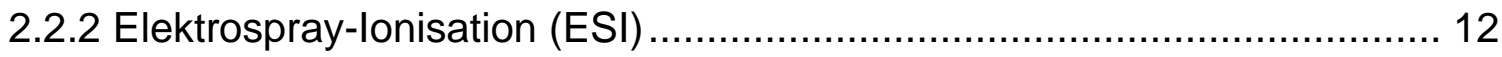

2.2.3 Quadrupol-Ionenfalle als Massenanalysator ........................................ 15

2.2.4 Quadrupol-Ionenfalle als chemischer Reaktor...................................... 17

2.3 Elektrische Konduktometrie ................................................................. 18

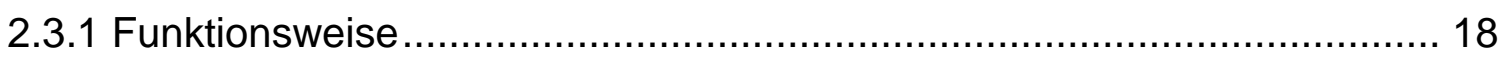

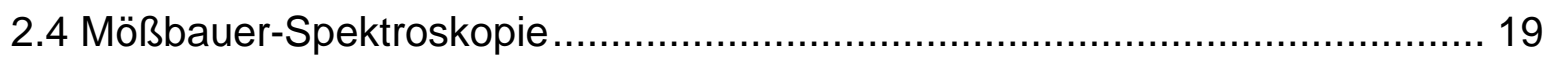

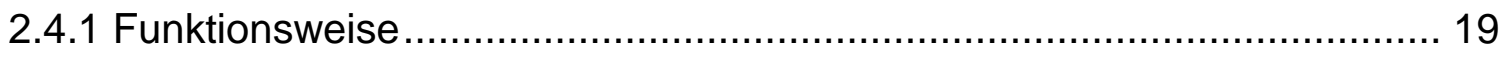

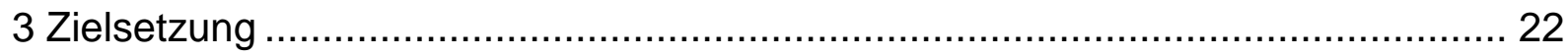

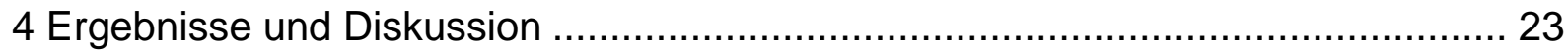

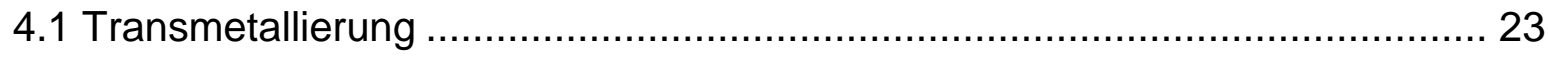

4.1.1 Transmetallierung in Abwesenheit von Additiven und Liganden .............. 23

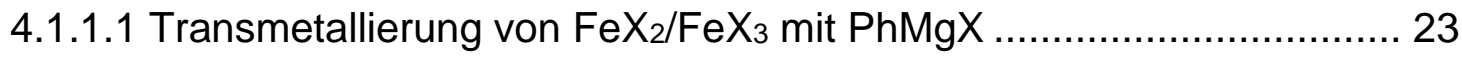

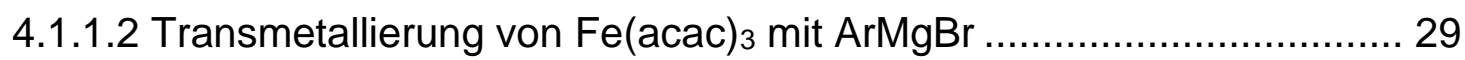

4.1.1.3 Transmetallierung von $\mathrm{Fe}(\mathrm{acac})_{3}$ mit $\mathrm{PhMgCl}$ und $\mathrm{RMgBr} \ldots \ldots \ldots \ldots \ldots . . . . .32$

4.1.1.4 Transmetallierung von $\mathrm{FeX}_{2} / \mathrm{FeX}_{3}$ mit MeMgX............................... 36

4.1.1.5 Transmetallierung von $\mathrm{Fe}(\mathrm{acac})_{3}$ mit AlkMgCl ............................... 39

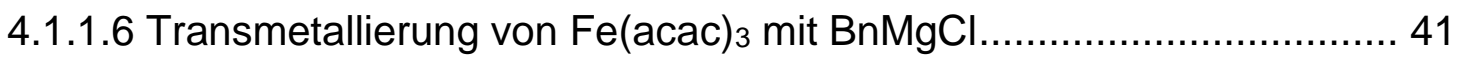


4.1.1.7 Transmetallierung von $\mathrm{Fe}(\mathrm{acac})_{3}$ mit $\mathrm{Me}_{3} \mathrm{SiCH}_{2} \mathrm{MgCl} \ldots \ldots \ldots \ldots \ldots \ldots \ldots . . . . . . . . .43$

4.1.1.8 Intermolekulare Austauschreaktionen ......................................... 45

4.1.1.9 Transmetallierung von $\mathrm{FeCl}_{2}$ oder $\mathrm{Fe}(\mathrm{acac})_{3}$ mit $\mathrm{RLi}$........................ 47

4.1.1.10 Transmetallierung von $\mathrm{FeCl}_{2}$ oder $\mathrm{Fe}(\mathrm{acac})_{3}$ mit $\mathrm{R}_{2} \mathrm{Zn}$................... 48

4.1.1.11 Bildung und Stabilität von Organoferraten ................................... 49

4.1.2 Transmetallierung in Anwesenheit von Additiven und Liganden .............. 54

4.1.2.1 Transmetallierung von $\mathrm{Fe}(\mathrm{acac})_{3}$ mit $\mathrm{ArMgX}$ in Anwesenheit von

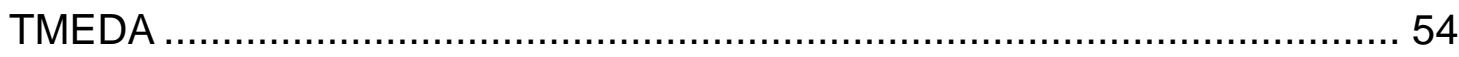

4.1.2.2 Transmetallierung von $\mathrm{Fe}(\mathrm{acac})_{3}$ mit AlkMgX in Anwesenheit von

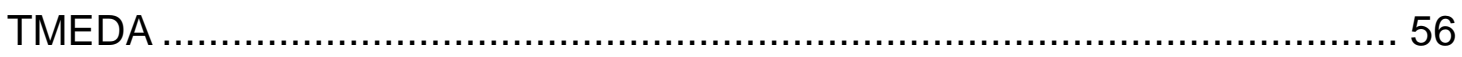

4.1.2.3 Transmetallierung von $\mathrm{FeCl}_{2}(\mathrm{dppbz})_{2}$ mit $\mathrm{ArMgX} \ldots \ldots \ldots \ldots \ldots \ldots \ldots \ldots \ldots . . . . . . . . . . . . .60$

4.1.2.4 Transmetallierung von $\mathrm{FeCl}_{2}(\mathrm{dppbz}) 2$ mit AlkMgCl .......................... 63

4.1.2.5 Transmetallierung von $\mathrm{Fe}(\mathrm{acac})_{3}$ mit $\mathrm{PhMgCl}$ oder $\mathrm{MeMgCl}$ in Anwesenheit weiterer Additive und Liganden ............................................. 64

4.1.2.6 Transmetallierung von $\mathrm{FeCl}_{2}$ mit $\mathrm{R}_{2} \mathrm{Zn}$ in Anwesenheit bidentater

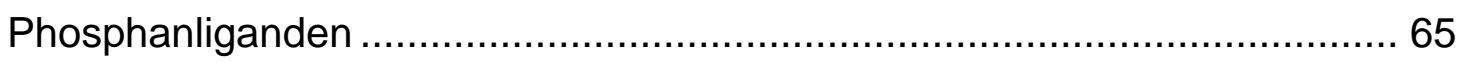

4.1.2.7 Einfluss von Additiven und Liganden auf die Transmetallierung ........ 66

4.2 Reaktivität gegenüber organischen (Pseudo)-Halogeniden ........................... 70

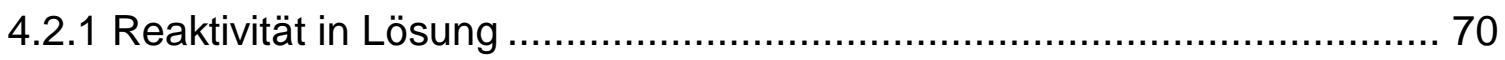

4.2.1.1 Reaktivität von Phenylferraten gegenüber R'X …........................... 70

4.2.1.2 Reaktivität von Methylferraten gegenüber R'X .............................. 74

4.2.1.3 Reaktivität von Butylferraten gegenüber R'X …............................ 77

4.2.1.4 Reaktivität von Benzylferraten gegenüber R'X.............................. 78

4.2.1.5 Reaktivität der Organoferrate gegenüber organischen Substraten in

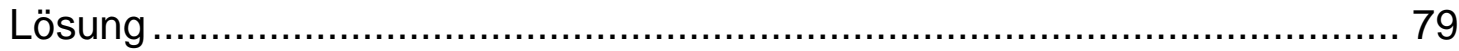

4.2.2 Bimolekulare Gasphasenreaktivität .................................................. 82 
4.2.2.1 Aufbau und Funktionsweise einer Apparatur zur Untersuchung bimolekularer Gasphasenreaktionen ............................................. 82

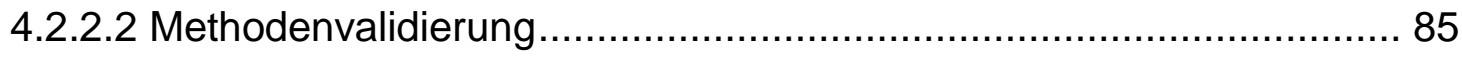

4.2.2.3 Bimolekulare Gasphasenreaktivität von Phenylferraten gegenüber R'X 89

4.3 Reduktive Eliminierung und Konkurrenzreaktionen .................................. 93

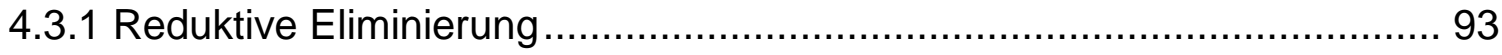

4.3.2 Freisetzung von Radikalen .......................................................... 102

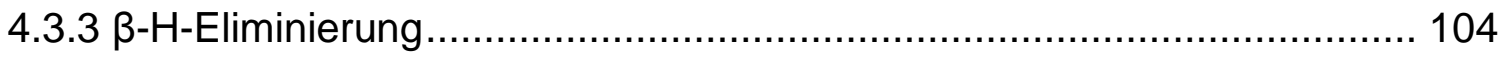

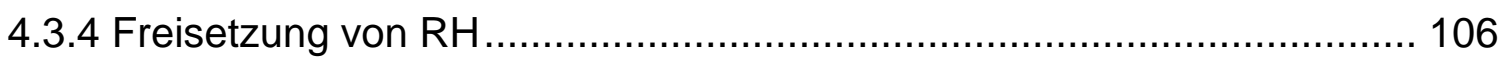

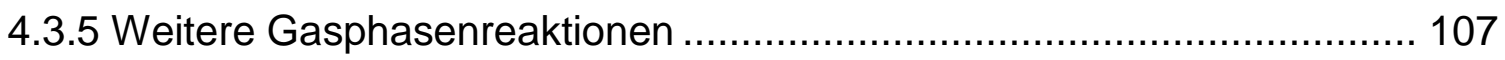

4.3.6 Unimolekulare Gasphasenreaktivität der Organoferrate im Vergleich .... 110

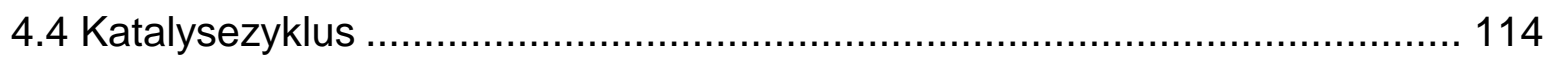

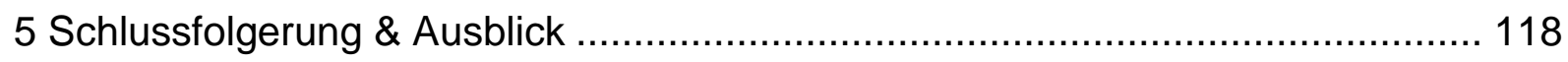

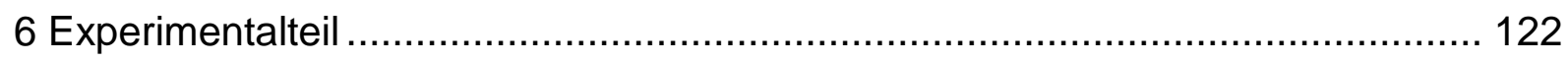

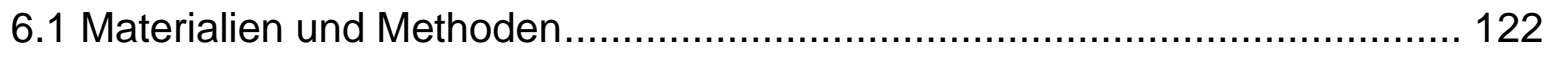

6.1.1 Allgemeine Arbeitstechniken und Methoden ................................... 122

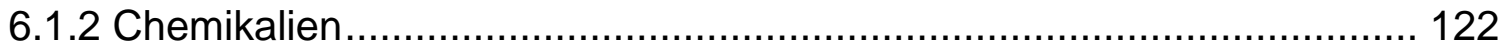

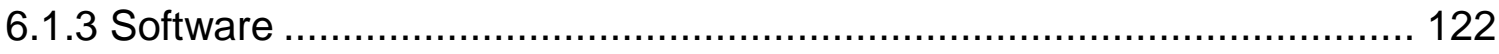

6.1.4 Komplexometrische Bestimmung von Eisen [] ................................. 123

6.1.5 Konzentrationsbestimmung metallorganischer Reagenzien ................. 123

6.2 Synthesen und Probenvorbereitung ........................................... 124

6.2.1 Synthese metallorganischer Reagenzien ................................... 124

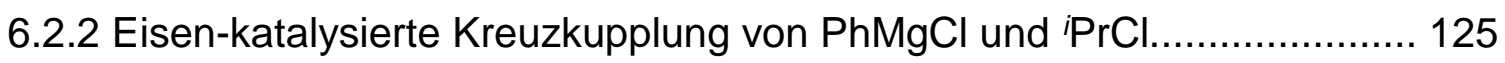

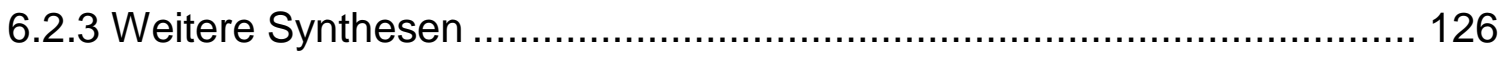

6.2.4 Synthese und Isolation eisenhaltiger Feststoffe ............................ 128 
6.2.5 Probenvorbereitung

6.3 Analytische Methoden ........................................................................ 130

6.3.1 ESI-Massenspektrometrie …….................................................... 130

6.3.2 Elektrische Konduktometrie .......................................................... 131

6.3.3 Mößbauer-Spektroskopie ............................................................ 131

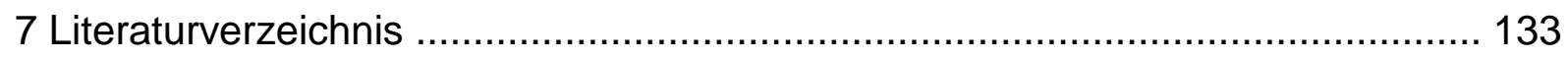






\section{Eisen-katalysierte Kreuzkupplungsreaktionen}

\subsection{Einführung}

Übergangsmetall-katalysierte Kreuzkupplungsreaktionen sind eine der gängigsten und effizientesten Synthesemethoden in der modernen organischen Chemie, um neue KohlenstoffKohlenstoff-Bindungen auszubilden ( $\rightarrow$ Schema 1). ${ }^{[1]}$ Speziell Verbindungen der Übergangsmetalle Palladium und Nickel haben sich hier als besonders aktive Katalysatoren erwiesen. ${ }^{[2]}$

$$
\mathrm{R}-\mathrm{M}+\mathrm{R}^{\prime}-\mathrm{X} \stackrel{[\text { Kat }]}{\longrightarrow} \mathrm{R}-\mathrm{R}^{\prime}+\mathrm{M}-\mathrm{X}
$$

Schema 1. Allgemeine Reaktionsgleichung für die Übergangsmetall-katalysierte Kreuzkupplungsreaktion zwischen Organometallreagenzien R-M und organischen (Pseudo)-Halogeniden R'-X. Palladium- und Nickelverbindungen stellen heutzutage die wichtigsten Katalysatorsysteme für diese Reaktionsklasse dar.

Trotz ihrer hohen Bedeutung in Kreuzkupplungen gehen mit der Verwendung dieser Katalysatorsysteme aber auch immer ökologische und ökonomische Nachteile einher. ${ }^{[3]}$ Aufgrund der zunehmenden Knappheit, der Toxizität sowie der hohen Kosten der zumeist verwendeten Übergangsmetalle stellt die Entwicklung neuer Katalysatorsysteme einen wichtigen Forschungsschwerpunkt dar. Vor allem Eisenverbindungen scheinen eine wünschenswerte Alternative $\mathrm{zu}$ sein, um eine kosteneffiziente und nachhaltige Synthesechemie zu gewährleisten. Eisen ist von allen Übergangsmetallen das Element mit dem mit Abstand höchsten natürlichen Vorkommen auf der Erde, was sich in einem im Vergleich zu Palladium relativ niedrigen Weltmarktpreis widerspiegelt. ${ }^{[4]}$

Typische in Kreuzkupplungsreaktionen genutzte Eisen-Präkatalysatoren weisen neben den geringeren Kosten im Vergleich mit gängigen Palladium- und Nickelverbindungen zudem weniger toxikologische Gefahren auf $(\rightarrow$ Tabelle 1$){ }^{[5]}$ Laut allgemein gültiger Gefahrstoffverordnung sind Eisenverbindungen im Vergleich toxikologisch weniger bedenklich und damit auch einfacher handhabbar. Somit würden bei der Verwendung von Eisenkatalysatoren, speziell in der pharmazeutischen Industrie, teure und zeitaufwendige Aufreinigungsprozesse entfallen, da Eisen aus toxikologischen Gründen nicht vollständig aus den Erzeugnissen entfernt werden müsste. Zudem ist Eisen das Übergangsmetall mit der größten biologischen Relevanz und damit vergleichsweise wenig belastend für Natur und Umwelt. ${ }^{[6]}$ 
Tabelle 1. Toxizität typischer Eisenverbindungen im Vergleich mit Palladiumchlorid und Nickelchlorid. ${ }^{[5]}$

\begin{tabular}{lcc}
\hline Verbindung & Gefahrstoffsymbole & LD $_{50} / \mathbf{~ m g ~ k g}^{-1}$ \\
\hline $\mathrm{Fe}(\mathrm{acac})_{3}$ & $\vdots$ & 1872 \\
$\mathrm{FeCl}_{3}$ & $\vdots$ \\
$\mathrm{FeCl}_{2}$ & \\
$\mathrm{PdCl}_{2}$ & & 1350 \\
$\mathrm{NiCl}_{2}$ & & 450 \\
\hline
\end{tabular}

Allgemein zeichnen sich Eisenkatalysatoren durch eine große Vielfalt und leichte Veränderbarkeit in den Oxidationsstufen aus. ${ }^{[7]}$ Zusätzlich zeigen sie eine ausgeprägte LewisAcidität. Damit eignen sie sich prinzipiell als Katalysatoren für eine Vielzahl von Reaktionsklassen, so auch für Kreuzkupplungsreaktionen. ${ }^{[8]}$

Eisen-katalysierte Kreuzkupplungen konnten bereits erfolgreich im Kilogramm-Maßstab durchgeführt werden..$^{[9]}$ Hier zeigt sich das hohe Potential von Eisen in dieser Reaktionsklasse, auch gegenüber anderen für die Kreuzkupplung interessanten 3d-Übergangsmetallen wie z. B. Cobalt. ${ }^{[10]}$ Das Potential von Eisen wird bisher aber nicht vollends ausgeschöpft. So bleibt Palladium in Kreuzkupplungsreaktionen nach wie vor das Metall der Wahl. ${ }^{[11]}$ Dies liegt vor allem an den bisher unzureichenden mechanistischen Erkenntnissen und der damit einhergehenden schlechten Steuerbarkeit der Eisen-katalysierten Kreuzkupplungsreaktion. So bleibt bis heute die Natur der in situ generierten, katalytisch aktiven Eisen-Spezies unbekannt. 


\subsection{Synthese: Potential und ungelöste Probleme}

In den frühen 40er-Jahren konnten Kharasch und Fields erstmals das Potential von Eisen in C-C-Kupplungsreaktionen durch die erfolgreiche Synthese biarylischer Homokupplungsprodukte aufzeigen. ${ }^{[12]}$ Annähernd 30 Jahre später gelang es Tamura und Kochi, die erste Eisen-katalysierte Kreuzkupplung zu entwickeln. ${ }^{[13]}$ In einer Reaktion von $\mathrm{MeMgBr}$ mit Vinylbromid in Anwesenheit katalytischer Mengen an $\mathrm{FeCl}_{3}$ konnte erfolgreich das Kreuzkupplungsprodukt gebildet werden ( $\rightarrow$ Schema 2 ).

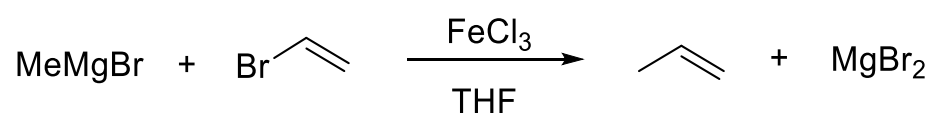

Schema 2. Eisen-katalysierte Kumada-Kreuzkupplung von $\mathrm{MeMgBr}$ und Vinylbromid in Anwesenheit von $\mathrm{FeCl}_{3}$.

Seit den Pionierarbeiten von Tamura und Kochi folgte eine Vielzahl weiterer Forschungsarbeiten, die in ihrer Gesamtheit darauf hindeuten, dass Eisenkatalysatoren, speziell in Kumada-Kreuzkupplungsreaktionen, eine effiziente Alternative zu gängigen Palladiumkatalysatoren sein können ( $\rightarrow$ Tabelle 2$)$.

Tabelle 2. Übersicht literaturbekannter, Eisen-katalysierter Kumada-Kreuzkupplungsreaktionen zwischen Grignard-Reagenzien RMgX und organischen (Pseudo)-Halogeniden R'X inklusive der eingesetzten [Fe]-Präkatalysatoren, Additive und Liganden.

\begin{tabular}{|c|c|c|c|c|}
\hline Eintrag & $\mathbf{R}=$ & $\mathbf{R}^{\prime}=$ & [Fe]-Präkatalysator & Additiv/Ligand \\
\hline $1^{[14]}$ & Alkyl & Alkyl & $\mathrm{Fe}(\mathrm{OAc})_{2}$ & $\mathrm{NHC}^{a}, \mathrm{dpp}^{b}$ \\
\hline $2 a^{[15]}$ & Alkyl & Aryl & $\mathrm{FeBr}_{2}, \mathrm{FeX}_{3}\left(\mathrm{X}=\mathrm{acac}^{c}, \mathrm{~F}\right)$ & $\mathrm{NHC}, \mathrm{NMP}^{d}$ \\
\hline $2 b^{[15 a, b, e, 16]}$ & Alkyl & Heteroaryl & $\mathrm{FeX}_{3}(\mathrm{X}=\mathrm{acac}, \mathrm{Cl})$ & NMP \\
\hline $2 c^{[17]}$ & Alkyl & Alkenyl & $\mathrm{FeCl}_{2}, \mathrm{FeX}_{3}(\mathrm{X}=\mathrm{acac}, \mathrm{Cl})$ & $\mathrm{LiCl}, \mathrm{NHC}, \mathrm{NMP}$ \\
\hline $3^{[18]}$ & Alkyl & Alkinyl & $\mathrm{Fe}(\mathrm{acac})_{3}$ & - \\
\hline $4 a^{[17 d, 19]}$ & Aryl & Alkyl & $\begin{array}{l}\mathrm{FeCl}_{2}, \mathrm{FeX}_{3}(\mathrm{X}=\text { acac, } \mathrm{Cl} \text {, } \\
\text { salen-Cl) }\end{array}$ & $\begin{array}{l}\text { NMP, dppx, } \\
\text { TMEDA }\end{array}$ \\
\hline $4 b^{[20]}$ & Aryl & Alkenyl & $\begin{array}{l}\mathrm{FeCl}_{2}, \mathrm{FeX}_{3}(\mathrm{X}=\text { acac, } \mathrm{Cl}, \\
\left.\mathrm{dbm}^{f}, \mathrm{~F}^{g}\right)\end{array}$ & $\begin{array}{l}\mathrm{DME}^{e}, \mathrm{NHC} \\
\text { TMEDA }\end{array}$ \\
\hline $4 c^{[20 t, 21]}$ & Aryl & Aryl & $\mathrm{Fe}(\mathrm{OTf})_{2}, \mathrm{FeX}_{3}\left(\mathrm{X}=\mathrm{F}^{f}, \mathrm{OR}\right)$ & $\mathrm{NHC}$ \\
\hline $4 d^{[22]}$ & Aryl & Heteroaryl & $\mathrm{FeX}_{3}(\mathrm{X}=\mathrm{acac}, \mathrm{Br}, \mathrm{Cl})$ & LiCl, NMP, dppx \\
\hline $5^{[23]}$ & Alkenyl & Alkyl & $\mathrm{Fe}(\mathrm{acac})_{3}$ & TMEDA \\
\hline $6^{[24]}$ & Alkinyl & Alkyl & $\mathrm{FeBr}_{2}$ & NMP \\
\hline $7^{[25]}$ & Alkinyl & Alkenyl & $\mathrm{FeCl}_{3}$ & $\mathrm{LiBr}$ \\
\hline
\end{tabular}

${ }^{a} \mathrm{NHC}=\mathrm{N}$-Heterocyclisches Carben. ${ }^{b} \mathrm{dppx}=$ bidentater Phosphanligand. ${ }^{c}$ acac $=$ Acetylacetonat. ${ }^{d} \mathrm{NMP}=$ $N$-Methyl-2-pyrollidon. ${ }^{e} \mathrm{DME}=1,2$-Dimethoxyethan. ${ }^{f} \mathrm{dbm}=$ Dibenzoylmethan. ${ }^{g}$ mit und ohne Kristallwasser (3 $\left.\mathrm{H}_{2} \mathrm{O}\right)$. 
Schon die bloße Anzahl an Publikationen verdeutlicht, dass Eisen-katalysierte KumadaKreuzkupplungsreaktionen von $\mathrm{sp}^{2}$ - mit $\mathrm{sp}^{3}$-Kohlenstoffzentren synthetisch besonders wertvoll sind ( $\rightarrow$ Tabelle 2, Einträge 2a-c, 4a), wohingegen die $\mathrm{sp}^{3}-\mathrm{sp}^{3}-$ Kreuzkupplung Eisenkatalysiert weitestgehend unzugänglich ist ( $\rightarrow$ Tabelle 2, Eintrag 1). Die Kreuzkupplung von arylischen Kohlenstoffzentren ist ohne Ligandsysteme Eisen-katalysiert nur schwer zu bewerkstelligen. Durch Zuhilfenahme eines Katalysators aus Eisen(III)fluorid und $N$-heterocyclischen Carbenen (oder davon abgeleiteten Katalysatorsystemen) sind aber auch diese Kreuzkupplungen heutzutage Eisen-katalysiert erfolgreich durchführbar ( $\rightarrow$ Tabelle 2, Eintrag 4c). Zuletzt konnten auch erste Erfolge im Bereich der Eisen-katalysierten KumadaKreuzkupplung von sp- mit sp²- oder $\mathrm{sp}^{3}$-Kohlenstoffzentren $(\rightarrow$ Tabelle 2, Einträge 3, 6, 7) und der enantioselektiven Darstellung von Kreuzkupplungsprodukten ${ }^{[26]}$ erzielt werden.

Aufgrund seiner Unempfindlichkeit gegenüber Oxidation und Hydrolyse ist $\mathrm{Fe}(\mathrm{acac})_{3}$ der gängigste Eisen-Präkatalysator. Zumeist verwendete Additive und Liganden sind $N$-Donorbasen wie TMEDA und NMP und bidentate Phosphanliganden dppx ( $\rightarrow$ Tabelle 2 ).

Außer in Kumada-Kreuzkupplungsreaktionen wurden Eisenkatalysatoren bereits in Negishi-[27] und Suzuki-Kreuzkupplungen ${ }^{[28]}$ mit großem Erfolg eingesetzt. Neben Organobor-Reagenzien konnten Suzuki-artige Kreuzkupplungsreaktionen auch mit weiteren OrganometallReagenzien aus der Gruppe 13 des Periodensystems erfolgreich durchgeführt werden. ${ }^{\text {[27g,29] }}$ Daneben sind alternative Kreuzkupplungsreaktionen von Organylhalogeniden mit weiteren

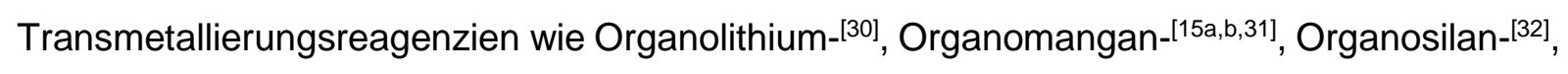
Organokupfer-[33] und in situ generierten Diaryltitanat-Reagenzien ${ }^{[34]}$ möglich. 


\subsection{Mechanismen: Erkenntnisse und offene Fragen}

Der Mechanismus der Eisen-katalysierten Kreuzkupplung von Organylhalogeniden oder Pseudohalogeniden mit Grignard-Reagenzien ist nach heutigem Forschungsstand noch unzureichend verstanden. Speziell die Oxidationsstufen der beteiligten Eisenintermediate wurden in der Vergangenheit kontrovers diskutiert. Die meisten Studien deuten auf eine mechanistische Beteiligung des Fe(I)/Fe(III)-[35] oder des Fe(II)/Fe(III)-Redoxpaares ${ }^{[26 b, 36]}$ hin, wobei auch die Beteiligung elektronenreicherer Oxidationsstufen von Eisen ${ }^{[20 c, 35 b, 37]}$ in der Vergangenheit bereits erwogen worden ist.

Der Mechanismus und die Struktur der eisenhaltigen Reaktionsintermediate sind stark abhängig von der Natur der organischen Reste am Grignard-Reagenz und am elektrophilen Substrat sowie von der Anwesenheit von Additiven und Liganden. Unabhängig von dieser Tatsache lassen sich die bisher gewonnenen mechanistischen Kenntnisse der Eisenkatalysierten Kumada-Kreuzkupplungsreaktion im Wesentlichen zu drei alternativen Katalysezyklen zusammenfassen. ${ }^{[38]}$ Der geläufigste und meist diskutierte Katalysezyklus $(\rightarrow$ Schema 3) zeigt die oxidative Addition eines elektrophilen Substrates an eine über Transmetallierung in situ gebildete organische Eisen(I)-Spezies. Der Additionsprozess kann dabei konzertiert (a) oder schrittweise (b) erfolgen. Der daraus gebildete Eisen(III)-Komplex setzt in einer reduktiven Eliminierung das Kreuzkupplungsprodukt frei und bildet die ursprüngliche Eisen(I)-Spezies zurück.

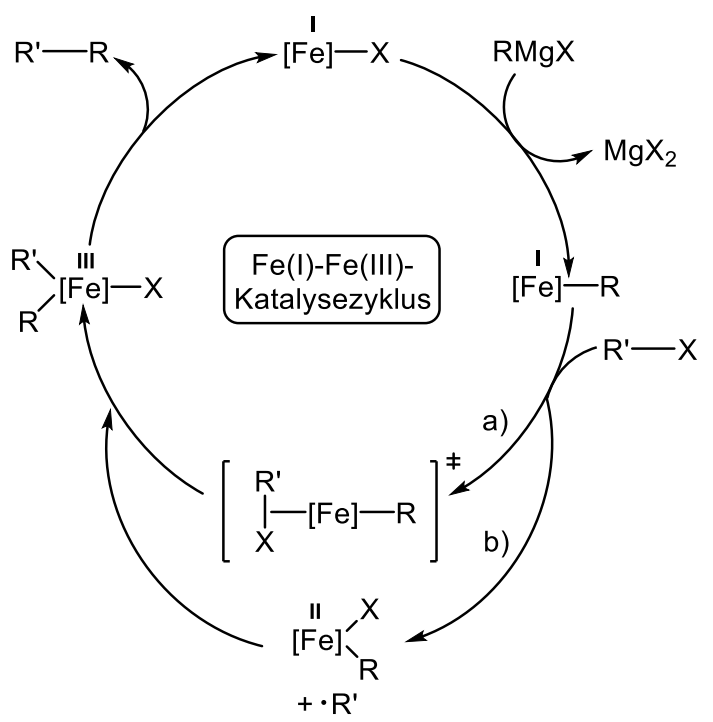

Schema 3. Postulierter $\mathrm{Fe}(\mathrm{I})$-Fe(III)-Katalysezyklus Eisen-katalysierter Kreuzkupplungsreaktionen über Transmetallierung, konzertierte (a) oder schrittweise (b) oxidative Addition und reduktive Eliminierung. ${ }^{[38]}$ 
Alternativ wurden Eisen-katalysierte Kreuzkupplungsreaktionen über einen Fe(II)-Fe(III)Mechanismus beschrieben ( $\rightarrow$ Schema 4, links). ${ }^{[38]}$ Eine über Transmetallierung in situ generierte, Organoeisen(II)-Spezies kann über Reaktion mit einem Substrat R'X ein Halogenatom abstrahieren. Das dabei entstehende Organylradikal kann anschließend in einem Rebound-Mechanismus mit der aus der Halogenabstraktion gebildeten Eisen(III)Spezies das Kreuzkupplungsprodukt unter Rückbildung der Ausgangsspezies freisetzen. Ausgehend von Organoeisen(II)-Spezies ist ein weiterer Mechanismus für Eisen-katalysierte Kumada-Kreuzkupplungsreaktionen denkbar ( $\rightarrow$ Schema 4, rechts). ${ }^{[38]}$ Wie im Mechanismus zuvor dargestellt, kann die organische Eisen(II)-Spezies ein Halogen von dem organischen Substrat abstrahieren. Das dabei freiwerdende Organylradikal kann alternativ zum zuvor beschriebenen Katalysezyklus mit einer weiteren Organoeisen(II)-Verbindung reagieren, sodass parallel zwei Eisen(III)-Spezies vorliegen. Im nachfolgenden Reaktionsschritt kann das Kreuzkupplungsprodukt aus dem heteroleptischen Eisen(III)-Komplex über reduktive Eliminierung freigesetzt werden. Über Komproportionierung von Eisen(I) und Eisen(III) mit anschließender Transmetallierung ist eine abschließende Katalysatorrückgewinnung unter Rückbildung der organischen Eisen(II)-Verbindung möglich.
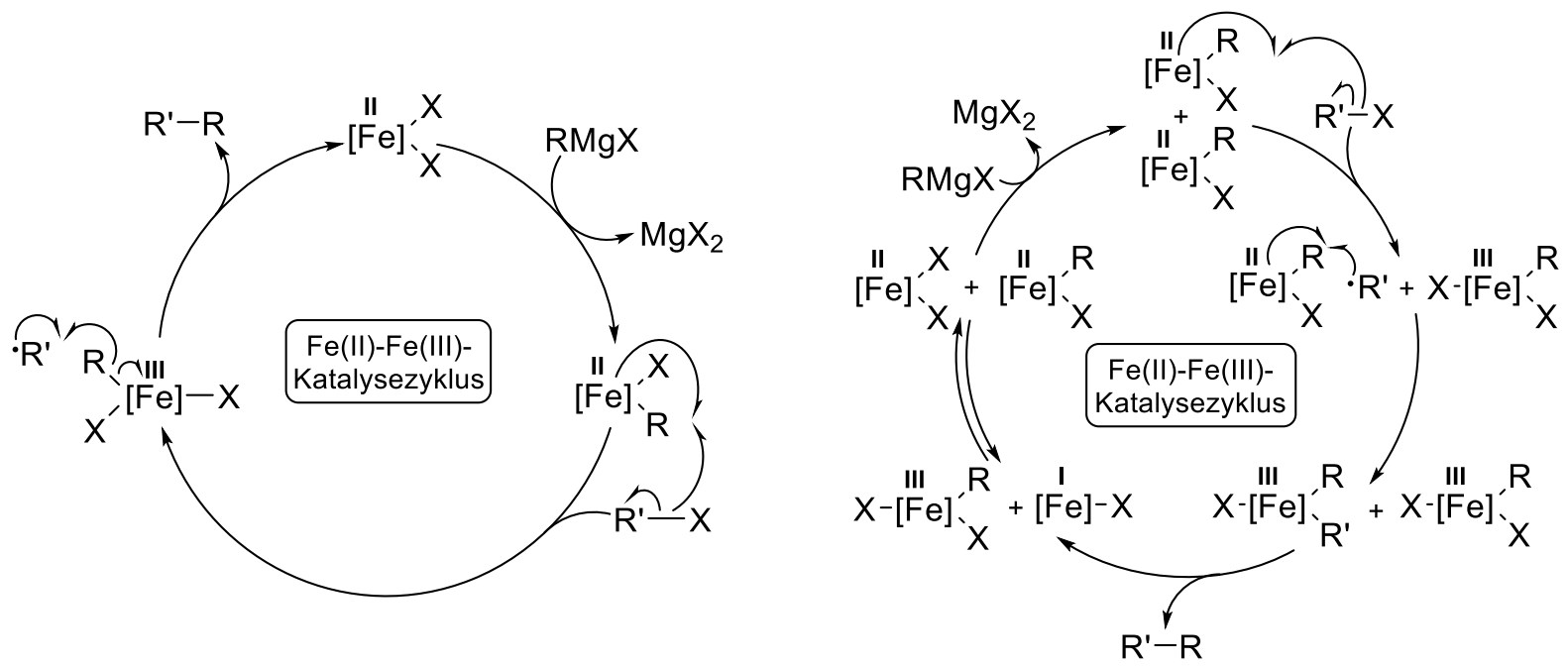

Schema 4. Links: Fe(II)-Fe(III)-Katalysezyklus Eisen-katalysierter Kumada-Kreuzkupplungsreaktionen über Transmetallierung, Halogenabstraktion und radikalischen Rebound-Mechanismus. Rechts: Fe(II)-Fe(III)-Katalysezyklus Eisen-katalysierter Kumada-Kreuzkupplungsreaktionen über Transmetallierung, bimolekulare Bindungshomolyse, reduktive Eliminierung und abschließende Komproportionierung. ${ }^{[38]}$ 
Unabhängig vom exakten Reaktionsmechanismus deuten die bisher gesammelten mechanistischen Erkenntnisse darauf hin, dass aus dem Eisen-Präkatalysator über Reduktion und Transmetallierung die katalytisch relevanten Eisen-Spezies entstehen, bevor diese in einem Additionsmechanismus mit dem organischen Substrat reagieren. ${ }^{[38]}$ Daher ist es von großer Bedeutung, herauszufinden, welche Eisen-Spezies in diesen Reaktionsschritten gebildet werden. So konnten in den letzten Jahren einige Organoeisen-Komplexe erfolgreich isoliert und mittels Röntgen-Kristallstrukturanalyse ${ }^{[35 b, 38,39]}$ charakterisiert werden (für eine Übersicht über die wichtigsten Vertreter dieser Substanzklasse, siehe Abbildung 1). Es zeigt sich, dass in Abwesenheit von Liganden neutrale Eisenspezies keine Rolle zu spielen scheinen. Vielmehr rücken vermehrt negativ geladene, Organoeisen-Komplexe, sogenannte Organoferrate, und deren Beteiligung in Eisen-katalysierten Kumada-Kreuzkupplungsreaktionen in den Fokus aktueller Forschungen.

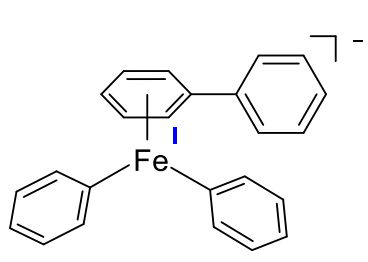

$\left[\mathrm{NBu}_{4}\right]^{+}$

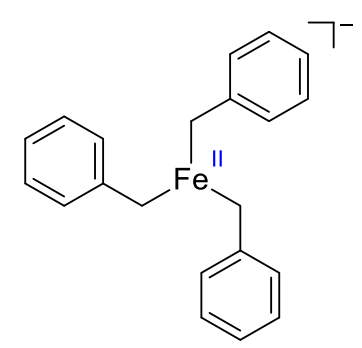

$\left[\mathrm{Mg}_{2} \mathrm{Cl}_{2}(\mathrm{OTf})(\mathrm{THF})_{3}\right]^{+}$

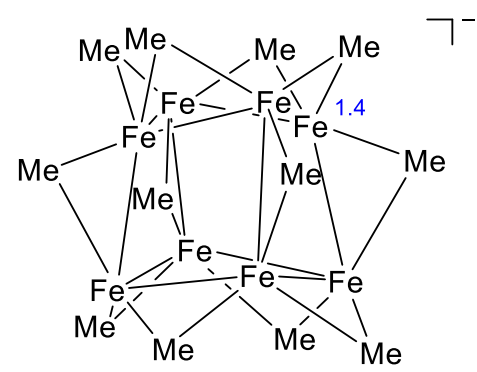

$\left[\mathrm{MgCl}(\mathrm{THF})_{5}\right]^{+}$

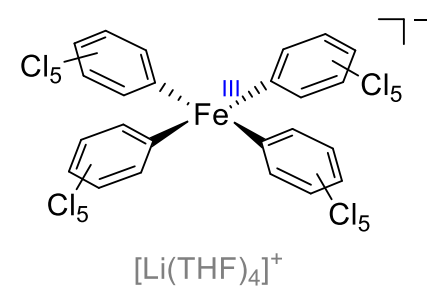

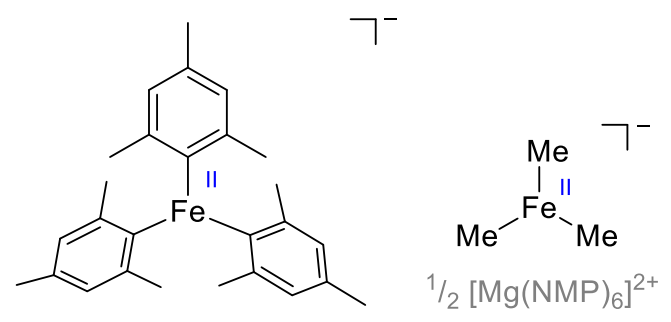

$\left[\mathrm{Mg}_{2} \mathrm{Br}_{3}(\mathrm{THF})_{6}\right]^{+}$

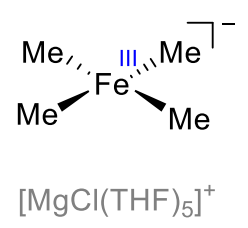

$\left[\mathrm{MgCl}(\mathrm{THF})_{5}\right]^{+}$

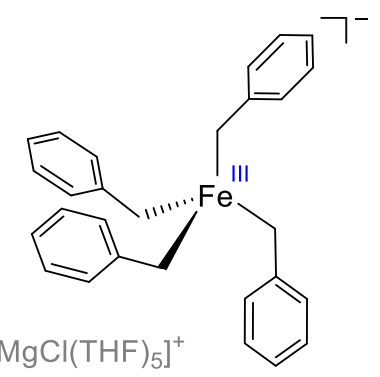

Abbildung 1. Übersicht über die wichtigsten Organoferrate, deren Struktur erfolgreich über RöntgenKristallstrukturanalyse ermittelt werden konnte. ${ }^{[38,39 d-h]}$

Die Reaktivität der Organoferrate gegenüber typischen Reaktionspartnern aus Eisenkatalysierten Kreuzkupplungsreaktionen wurde bisher nur sporadisch untersucht. Stöchiometrische Reaktivitätsstudien von Hu und Mitarbeitern zeigten, dass der Eisen(I)Komplex $\mathrm{Ph}_{2} \mathrm{Fe}\left(\eta^{6}-\mathrm{Ph}_{2}\right)^{-}$mit aliphatischen und aromatischen Bromiden reagierte und das entsprechende Kreuzkupplungsprodukt in geringen Ausbeuten bildete. ${ }^{[39 h]}$ Andere Studien 
bewiesen, dass auch die Organoferrate $\mathrm{Mes}_{3} \mathrm{Fe}(\mathrm{II})^{-}$, $\mathrm{Me}_{3} \mathrm{Fe}(\mathrm{II})^{-}$und $\mathrm{Bn}_{3} \mathrm{Fe}(\mathrm{II})^{-}$, in stöchiometrischen Mengen eingesetzt, mit Organylbromiden die entsprechenden Kreuzkupplungsprodukte ausbilden. ${ }^{[39 e, i]}$ Im Gegensatz dazu zeigten Untersuchungen von Neidig und Mitarbeitern, dass Kreuzkupplungsreaktionen von $\mathrm{Me}_{12} \mathrm{Fe}_{8}{ }^{-}$mit $\beta$-Bromstyrol nur in Gegenwart zusätzlicher Äquivalente an $\operatorname{MeMgBr}$ (1.25 Äq.) erfolgreich abliefen. ${ }^{[39 g]}$ 


\section{Analytische Methoden}

\subsection{Nachweis Eisen-organischer Spezies}

Um den Nachweis und die Analyse Eisen-organischer Spezies zu gewährleisten, bedarf es physikalischer und chemischer Analysetechniken, die unter inerten Bedingungen arbeiten und damit ungewollte Hydrolyse- und Oxidationsreaktionen dieser äußerst labilen Spezies auf ein Minimum beschränken. Im Allgemeinen ist die Röntgen-Kristallstrukturanalyse eine etablierte Methode, um metallorganische Intermediate strukturell zu beschreiben. Wie zuvor geschildert, konnten auf diese Art und Weise verschiedene Organoferrate erfolgreich charakterisiert werden (siehe Kapitel 1.3). ${ }^{[38,39 d-h]}$ Allerdings ist die Röntgen-Kristallstrukturanalyse in der Regel eine statische Methode. Sie liefert nur eine einzige Festkörperstruktur und missachtet dabei die mikroskopische Reaktivität und das dynamische Verhalten der Spezies in Lösung. Hingegen lassen sich durch Anwendung der Kernspinresonanzspektroskopie (nuclear magnetic resonance, NMR) die Struktur und das dynamische Verhalten metallorganischer Verbindungen in Lösung direkt beschreiben. Jedoch ist diese Analysetechnik größtenteils auf diamagnetische Verbindungen beschränkt. Der Nachweis paramagnetischer Komplexe, wie sie für Eisen-organische Spezies zu erwarten sind, ist mit NMR-Spektroskopie nicht unmöglich, resultiert jedoch häufig in nur schwer zu interpretierenden Messergebnissen. Für $\mathrm{Mes}_{3} \mathrm{Fe}(\mathrm{II})^{-}$als stabilem Organoferrat mit einem diamagnetischen Charakter fand die NMRSpektroskopie bereits Anwendung. ${ }^{[39 e]}$ Für den Nachweis paramagnetischer OrganoeisenSpezies bietet sich die Elektronenspinresonanz-Spektroskopie (ESR) an, da für den Einsatz dieser Methode paramagnetische Verbindungen zwingend Voraussetzung sind. Ohne zusätzliche Informationen über Struktur und Stöchiometrie der untersuchten Verbindungen reichen ESR-Daten allein zumeist nicht aus, um metallorganische Intermediate eindeutig zu charakterisieren. So haben Neidig und Mitarbeiter ESR-Spektroskopie genutzt, um das polynukleare Organoferrat $\mathrm{Me}_{12} \mathrm{Fe}_{8}{ }^{-}$zu beschreiben. ${ }^{[39 g]}$ Doch nur in Kombination mit weiteren Analysetechniken wie der Röntgen-Kristallstrukturanalyse war das resultierende ESRSpektrum sinnvoll interpretierbar. Eine ähnliche Problematik ist für die Untersuchung metallorganischer Intermediate mit UV/Vis- und Infrarotspektroskopie zu erwarten, da auch diese Methoden zu Messergebnissen führen, die ohne weitere Informationen über Struktur und Stöchiometrie der analysierten Verbindungen nur schwer zu deuten sind. Hingegen sind Untersuchungen mit Elektrospray-Ionisations-Massenspektrometrie (ESI-MS) geeignet, um einen direkten Zugang zur Stöchiometrie metallorganischer Komplexe und den Oxidationsstufen der beteiligten Metallzentren zu erhalten. ${ }^{[40]}$ Auch die Anwendung der 
${ }^{57} \mathrm{Fe}-$ Mößbauer-Spektroskopie erlaubt Rückschlüsse auf die Oxidationsstufen vorhandener Eisen-Zentren und bietet zusätzlich die Möglichkeit, deren Spinzustände zu ermitteln. Um Eisen-organische Spezies auch auf mikroskopischer Ebene in einem solvensfreien Raum qualitativ und quantitativ zu beschreiben und ihre intrinsische Reaktivität zu untersuchen, bieten sich Gasphasen-Analysetechniken an. ${ }^{[40]} \mathrm{Im}$ Idealfall lassen sich so einzelne Elementarschritte innerhalb eines zugrunde liegenden Reaktionsmechanismus direkt verfolgen, wobei störende Einflüsse durch Gegenionen oder Lösungsmitteleffekte ausgeschlossen werden können.

Es zeigt sich, dass eine Analysemethode allein nicht ausreicht, um Eisen-organische Spezies vollständig zu charakterisieren. Im Rahmen dieser Arbeit soll deswegen eine Kombination verschiedener Analysemethoden bestehend aus ESI-Massenspektrometrie und Gasphasentechniken sowie elektrischer Konduktometrie und ${ }^{57} \mathrm{Fe}-M o ̈ ß b a u e r-S p e k t r o s k o p i e$ eingesetzt werden. Diese Techniken wurden bisher noch nicht oder nur vereinzelt zum Nachweis und zur Charakterisierung Eisen-organischer Intermediate in KumadaKreuzkupplungsreaktionen genutzt. ${ }^{[39 g, 41]}$ 


\subsection{ESI-Massenspektrometrie}

\subsubsection{Aufbau eines Massenspektrometers}

Allgemein sind Massenspektrometer aus einem Probeneinlass, einer lonenquelle zur Erzeugung von Gasphasen-lonen, einem Massenanalysator zur lonenseparierung anhand ihres Masse-zu-Ladung-Verhältnisses $(\mathrm{m} / \mathrm{z})$ und einem Detektor zum Nachweis der lonen aufgebaut ( $\rightarrow$ Abbildung 2). ${ }^{[42]}$ Zum Betrieb eines Massenspektrometers ist zusätzlich ein Vakuumsystem nötig, das, abhängig vom Gerätetyp, im Hochvakuum- (HV) bis Ultrahochvakuumbereich (UHV) arbeitet. Elektrooptische Transferelemente (elektrische Linsen) zwischen den einzelnen Komponenten dienen der lonenfokussierung und -weiterleitung. ${ }^{[42,43]}$ Die Steuerung und Datenerfassung erfolgen computergestützt. Der Computer erzeugt auf Grundlage der Daten ein Massenspektrum, in dem die relative Intensität der lonen in Abhängigkeit von ihrem $\mathrm{m} / \mathrm{z}$-Verhältnis grafisch aufgetragen wird. Neben der Masse können auch die Isotopenmuster der lonen aus einem Massenspektrum entnommen werden, die oft einen wichtigen Beitrag zu deren Identifikation liefern.

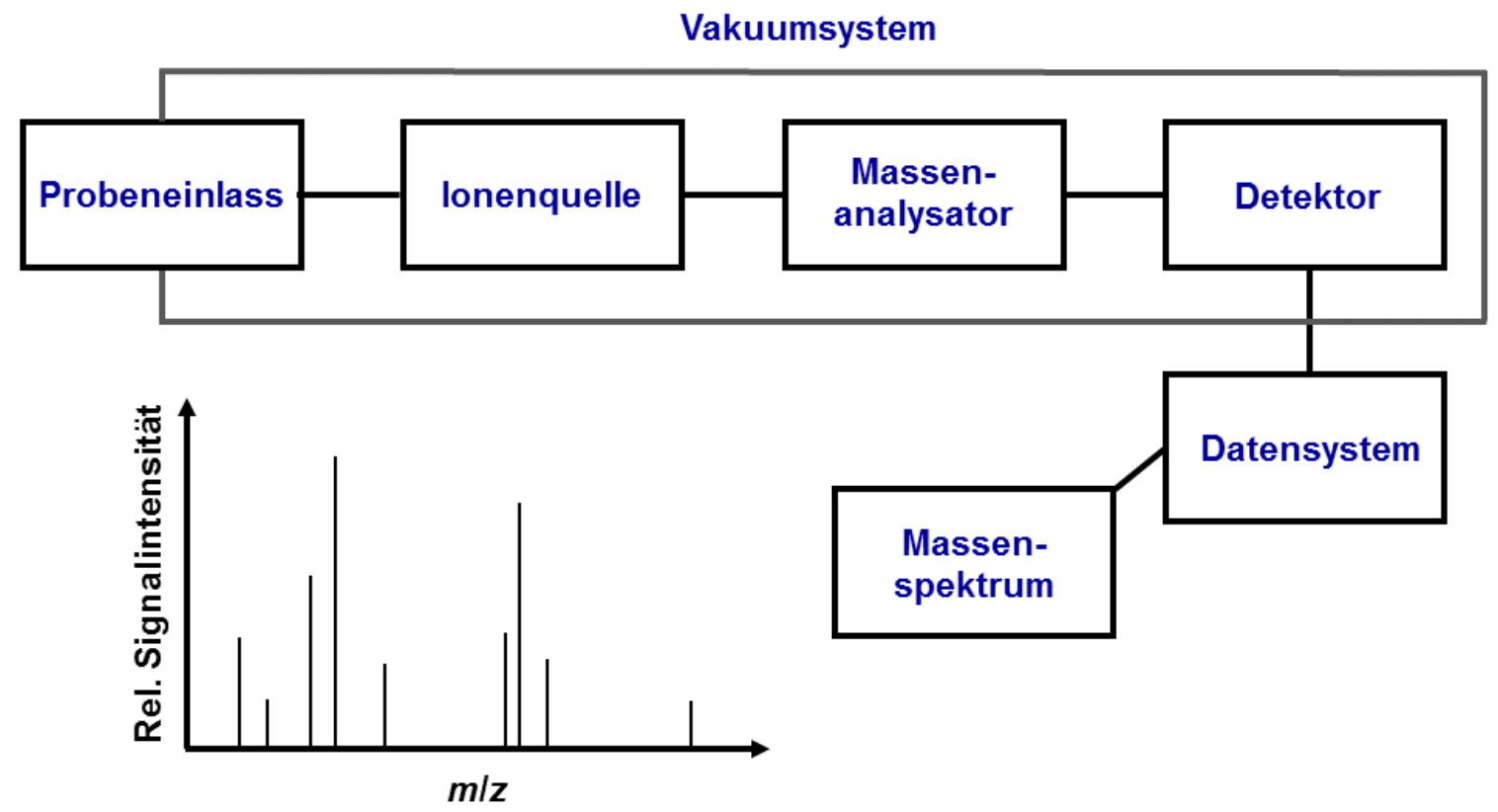

Abbildung 2. Schematischer Aufbau eines Massenspektrometers. Die Durchführung massenspektrometrischer Untersuchungen liefert ein Massenspektrum, in dem die relativen Intensitäten der lonen in Abhängigkeit von ihrem $\mathrm{m} / \mathrm{z}$-Verhältnis grafisch aufgetragen sind. ${ }^{[42]}$ 


\subsubsection{Elektrospray-Ionisation (ESI)}

Eine gängige lonisationstechnik, die in der Massenspektrometrie ihre Anwendung findet, ist die Elektrospray-Ionisation. Die Grundlage dieser Technik wurde in den 60er Jahren von Dole und Mitarbeitern geschaffen. ${ }^{[44]}$ Im Jahre 1984 machte John B. Fenn diese Ionisationsmethode letztendlich praktikabel und alltagstauglich. ${ }^{[45]}$ Sie stellt eine sehr schnelle und sensitive Technik dar, mit deren Hilfe es möglich ist, ein breites Spektrum an empfindlichen Analyten ohne ein hohes Maß an Fragmentierung von der flüssigen Phase in die Gasphase zu überführen und anschließend zu untersuchen. ${ }^{[42]}$ Zur Anwendung wird eine verdünnte Lösung des Analyten durch eine Metallkapillare gepumpt, an deren Ende eine Hochspannung (2-5 kV) angelegt ist ( $\rightarrow$ Abbildung 3) ${ }^{[46]}$ Das Vorzeichen der Spannung kann dabei positiv oder negativ sein, je nachdem, ob die Detektion von Kationen oder Anionen Ziel des Experimentes ist. Zwischen der Metallkapillare und einer Gegenelektrode wird ein elektrischer Feldgradient aufgebaut, der zu einer Ladungsseparation an der Oberfläche der Probenflüssigkeit führt. Als Folge bildet diese an der Kapillarenspitze die charakteristische Form eines Taylor-Konus aus. Sobald die Flüssigkeit das sogenannte Rayleigh-Limit überschreitet, werden von der Spitze des Taylor-Konus kleine Tröpfchen mit einem Überschuss an positiver oder negativer Ladung freigesetzt. Auf dem Weg zum Masseneinlass verlieren die Tröpfchen durch Verdampfung Lösungsmittelmoleküle, bis es letztendlich zur Freisetzung von Analyt-lonen kommt.

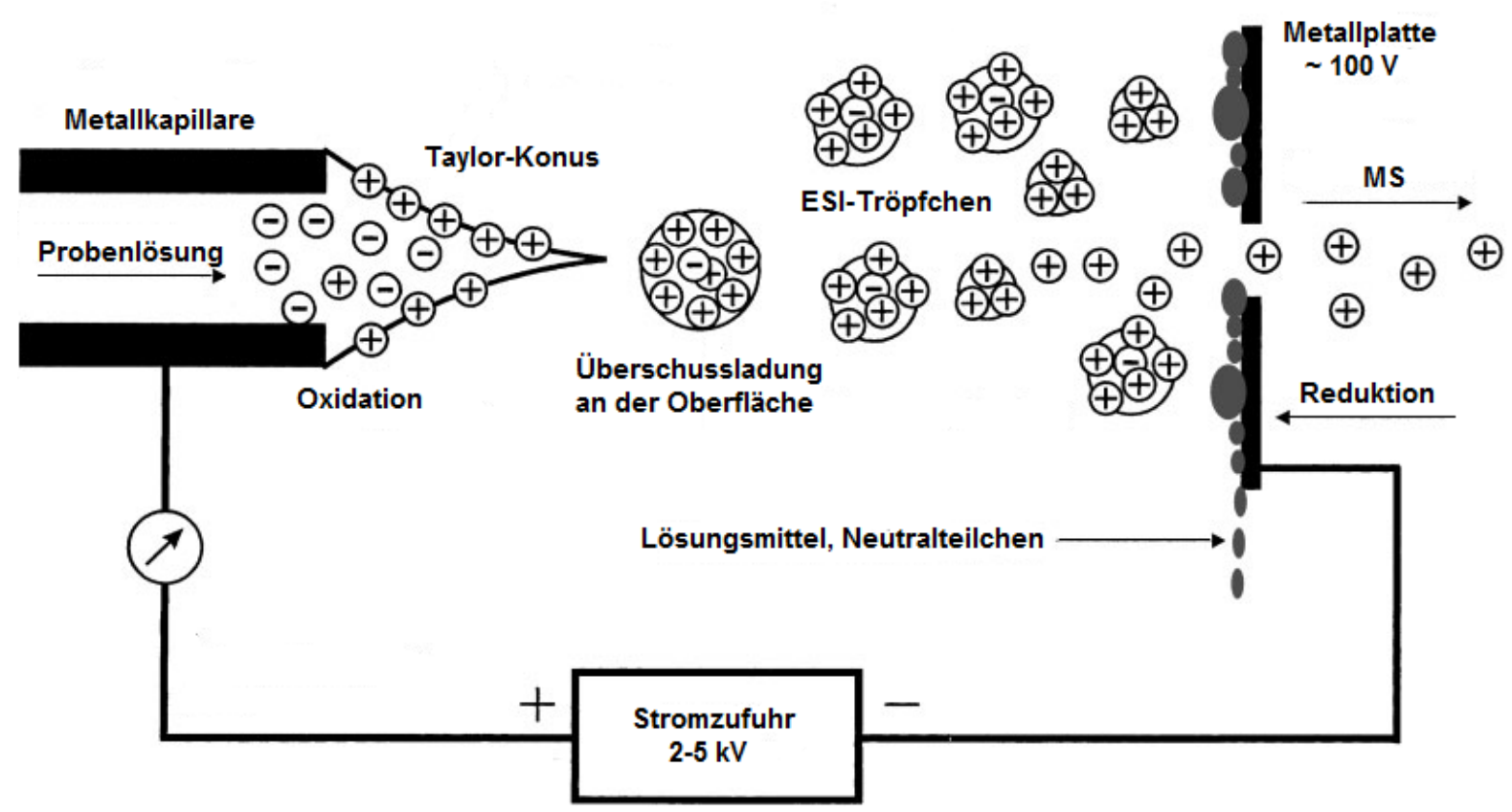

Abbildung 3. Schematischer Ablauf des ESI-Prozesses. Die Probenlösung wird durch eine Metallkapillare gepumpt, an der eine Hochspannung (2-5 kV) anliegt. Zwischen Kapillare und Gegenelektrode (Metallplatte) baut sich ein elektrischer Feldgradient auf. Als dessen Resultat bildet sich am Kapillarenende aus der Probenlösung ein Taylor-Konus mit einem Überschuss an Ionen an der Oberfläche. ESI-Tröpfchen werden aus Taylor-Konus herausgelöst, wandern zum Masseneinlass und setzen durch Lösungsmittelverdampfung Analyt-Ionen frei. ${ }^{[46]}$ 
Der Mechanismus der Überführung von Analyt-Ionen aus den ESI-Tröpfchen in die Gasphase ist bis heute nicht vollständig aufgeklärt. ${ }^{[4]}$ Mögliche Erklärungsansätze ( $\rightarrow$ Abbildung 4 ) bieten das Modell des geladenen Rückstandes (Charge Residue Model, CRM) ${ }^{[44]}$ und das Ionenverdampfungsmodell (Ion Evaporation Model, IEM). ${ }^{[48]}$ Beim CRM, das seine Gültigkeit vor allem für Makromoleküle besitzt, erfolgt eine Kombination aus Coloumb-Explosionen und Verdampfung von Lösungsmittelmolekülen bis zu dem Punkt, an dem die ESI-Tröpfchen durchschnittlich nur noch ein Analyt-Molekül aufweisen. ${ }^{[46,49]}$ Beim lonenverdampfungsmodell hingegen werden die ESI-Tröpfchen durch Verdampfen von Lösungsmittelmolekülen auf einen Radius von durchschnittlich 10-20 nm reduziert. Übersteigt die Coloumb-Abstoßung die Oberflächenspannung, erfolgt eine direkte Freisetzung von Analyt-lonen von der Oberfläche der ESI-Tröpfchen in die Gasphase. Dieses Modell gilt insbesondere für kleine und mittelgroße Analyt-Moleküle.

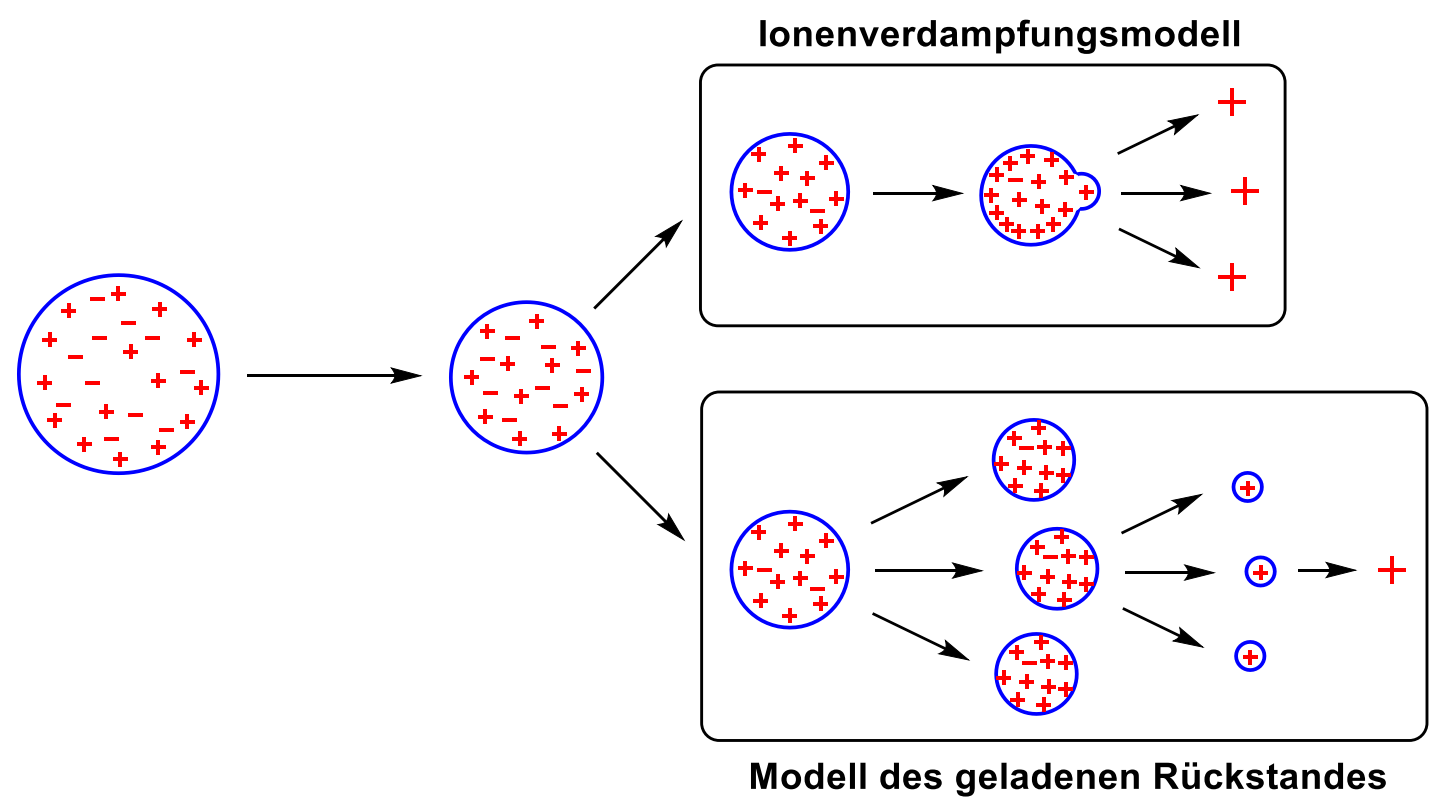

Abbildung 4. Zwei Modelle, die die Bildung von Gasphasen-lonen während des ESI-Prozesses erklären: Das lonenverdampfungsmodell (Ion Evaporation Model, IEM) und das Modell des geladenen Rückstandes (Charge Residue Model, CRM).[49]

Neben kleinen anorganischen und organischen Molekülen können mithilfe von ESIMassenspektrometrie eine Vielzahl an Analyten wie Proteine ${ }^{[50]}$, Oligonukleotide ${ }^{[50 a, 51]}$, Polymere $^{[52]}$ und Naturstoffe ${ }^{[53]}$ nachgewiesen werden. Auch im Bereich der metallorganischen Chemie und Katalysechemie kann ESI als sanfte lonisierungstechnik in Kombination mit Massenspektrometrie wichtige Erkenntnisse liefern. ${ }^{[40]}$ So lassen sich ionische Verbindungen der Hauptgruppen- und Übergangsmetalle, die in Lösung häufig schwach gebundene 
Liganden aufweisen, mit dieser Methode analysieren. ${ }^{[54]}$ Koszinowski und Mitarbeiter konnten in den letzten Jahren eine Vielzahl Metallat-organischer Komplexe erfolgreich mittels ESIMassenspektrometrie nachweisen. ${ }^{[5]}$

Im Vergleich mit anderen analytischen Methoden bietet die ESI-Massenspektrometrie einen direkten Zugang zu der Stöchiometrie und damit zu den Oxidationszahlen geladener Analyten. ${ }^{[46,56]}$ Problematisch hierbei ist, dass der ESI-Prozess über ungewollte Redoxreaktionen die Oxidationsstufen des Analyten dauerhaft verändern und damit zur Ausbildung und Detektion von Artefakt-lonen beitragen kann. ${ }^{[57]}$ Zudem lassen sich Neutralspezies mit dieser Methode nicht direkt nachweisen. Wird ESI als lonisierungstechnik in der Tandem-Massenspektrometrie verwendet, ermöglicht dies eine Vielzahl an weiteren Möglichkeiten, um Analyt-Ionen nicht nur zu identifizieren, sondern auch in ihrer unimolekularen und bimolekularen Reaktivität zu charakterisieren (siehe Kapitel 2.2.4). ${ }^{[58]}$ Quantitative Aussagen können mit ESI-Massenspektrometrie nur bedingt getroffen werden, da Faktoren wie die unbekannte ESI-Aktivität der Analyten Rückschlüsse auf die in Lösung vorliegenden Verhältnisse erschweren. ${ }^{[46]}$ Zudem ist es möglich, dass durch den ESI-Prozess Artefakt-lonen entstehen, die ursprünglich in Lösung nicht vorliegen. Daher ist es zwingend notwendig, ESI-massenspektrometrische Experimente durch alternative Analysemethoden zu validieren und damit Artefakt-Ionen aufzudecken oder endgültig auszuschließen. 


\subsubsection{Quadrupol-Ionenfalle als Massenanalysator}

Ein Quadrupol ist aus vier Stabelektroden mit hyperbolischem oder kreisförmigem Querschnitt aufgebaut, die gegenüberliegend in der $x$-/y-Ebene angeordnet sind und sich in $z$-Richtung erstrecken ( $\rightarrow$ Abbildung 5). ${ }^{[46,56]}$ An ihnen ist eine Spannung aus Gleichspannungs- und Wechselspannungsanteilen angelegt, wobei die jeweils gegenüberliegenden Stabelektroden dieselbe Spannungsart aufweisen. Ionen treten in z-Richtung in das Quadrupol ein. Aus dieser erfahren sie eine anziehende Kraft und abhängig vom Wechselspannungsanteil eine alternierende Kraft aus $x$ - und $y$-Richtung. Analyt-lonen, die in $x$-, $y$ - und $z$-Richtung stabile Trajektorien aufweisen, passieren das Quadrupol und erreichen den Detektor, ohne die Stabelektroden zu berühren. Für jede Gleichspannung, Wechselspannung und Frequenz besitzen lonen eines spezifischen $\mathrm{m} / \mathrm{z}$-Verhältnisses stabile Trajektorien. Nur diese können in der Folge das Quadrupol passieren und abschließend detektiert werden. In der Massenspektrometrie werden Quadrupole als robuste, kostengünstige und einfach handhabbare Massenanalysatoren mit einem vergleichsweise geringen Auflösungsvermögen eingesetzt.

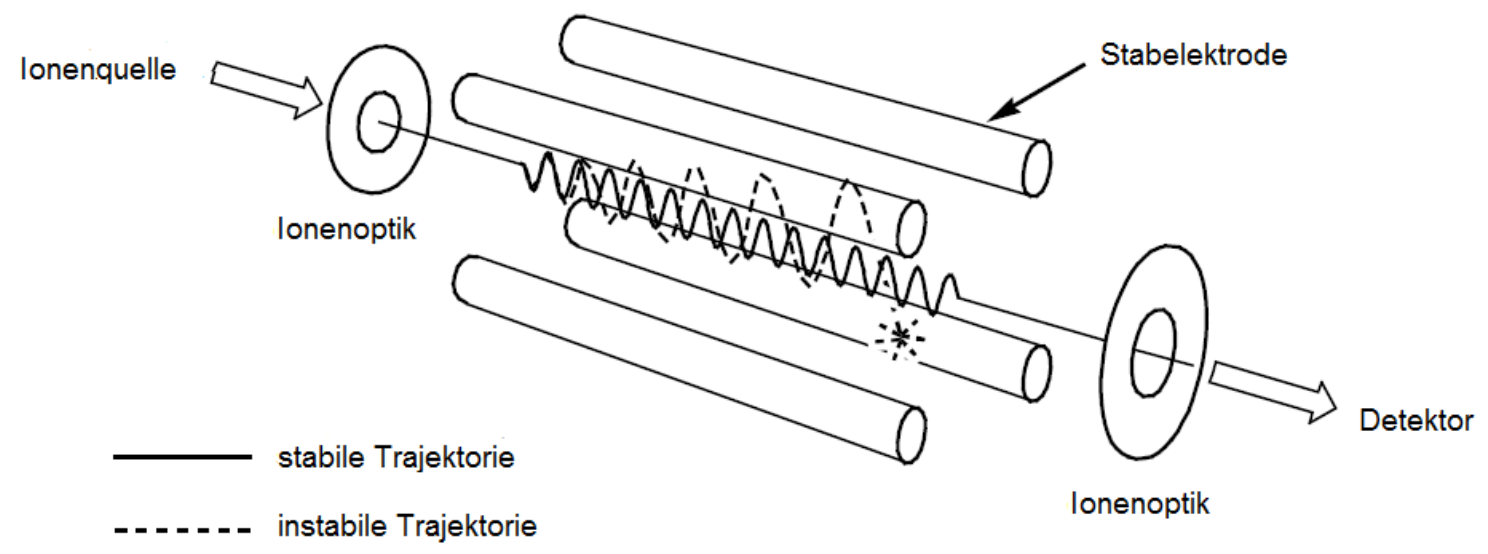

Abbildung 5. Aufbau eines Quadrupols als Massenanalysator. Die lonen aus einer lonenquelle passieren das elektrische Feld in einem Quadrupol, wenn sie stabile Trajektorien aufweisen. Optische Elemente vor und hinter dem Quadrupol dienen der lonenfokussierung. ${ }^{[56]}$

Quadrupole können zum Aufbau dreidimensionaler Quadrupol-lonenfallen genutzt werden $\left(\rightarrow\right.$ Abbildung 6). ${ }^{[46,56]}$ Allgemein sind diese aus einer Ringelektrode und zwei zylindrischen Stabelektroden mit hyperbolischem Querschnitt aufgebaut, wobei sich die Endkappen der letztgenannten oberhalb und unterhalb von der Ringelektrode befinden. Durch eine der Endkappen treten die Ionen in die Ionenfalle ein. Mit Hilfe eines Inertgases (typischerweise 
Helium) wird die Translationsbewegung und damit einhergehend die kinetische Energie der Ionen durch Stöße reduziert. Dies ermöglicht ein Ausharren der geladenen Spezies in dem durch Gleichspannungs- und Wechselspannungsanteile erzeugten elektrischen Feld der Ionenfalle. Ionen mit stabilen Trajektorien verweilen in der lonenfalle, wohingegen AnalytIonen mit instabilen Trajektorien durch axialen Auswurf oder durch Stöße mit der Wand verworfen werden. Trotz des in Relation eher geringen Auflösungsvermögens stellen dreidimensionale Quadrupol-lonenfallen heutzutage gängige Massenanalysatoren dar, die aufgrund ihrer kleinen und kompakten Form vergleichsweise geringe Anforderungen an das Vakuumsystem stellen. Aus der Möglichkeit, bestimmte lonen einzufangen und zu speichern, resultiert nicht nur eine hohe Nachweisempfindlichkeit dieses Massenanalysators, sondern es bietet sich auch die Möglichkeit, isolierte lonen bezüglich ihrer unimolekularen und bimolekularen Reaktivität zu charakterisieren.

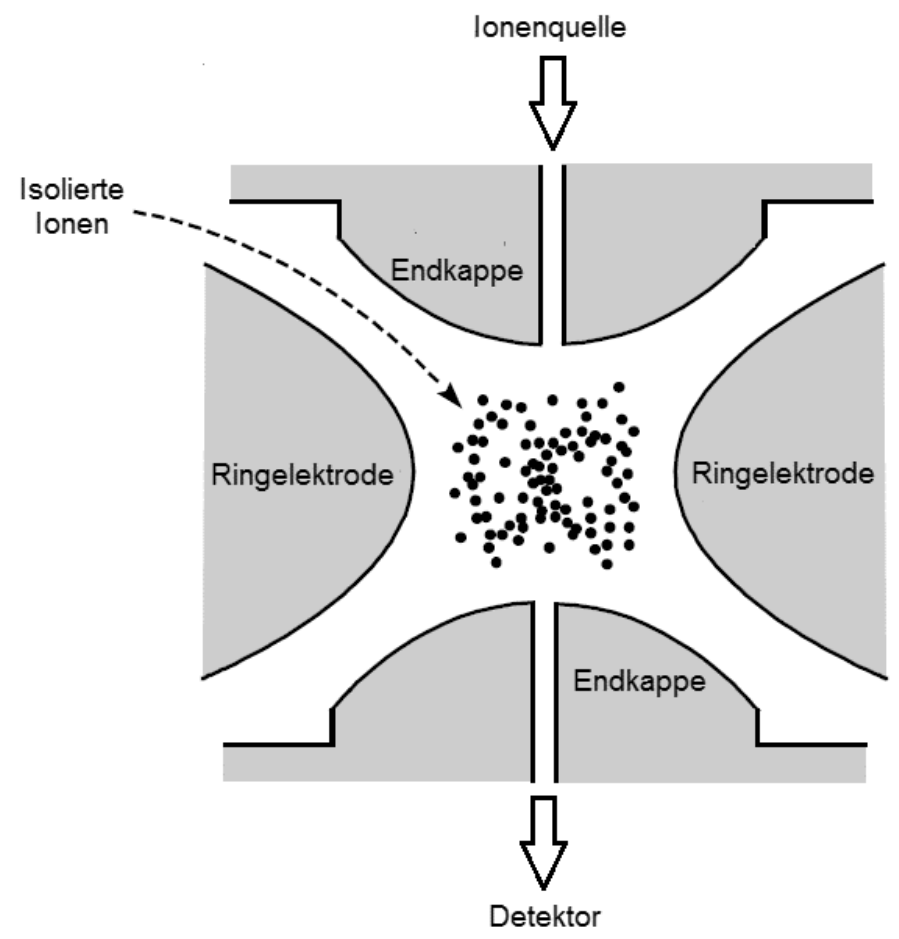

Abbildung 6. Aufbau einer dreidimensionalen Quadrupol-Ionenfalle zur Selektion und Speicherung von Ionen. ${ }^{[56]}$ 


\subsubsection{Quadrupol-Ionenfalle als chemischer Reaktor}

Quadrupol-Ionenfallen bieten die Möglichkeit, massenselektierte lonen durch stoßinduzierten Zerfall (collision induced dissociation, CID) zu untersuchen. ${ }^{[42,56,59]}$ Die isolierten Analyt-lonen werden in einer Stoßzelle durch Anlegen einer Spannung $V_{\text {Anr }}$ resonant angeregt. Als Folge stoßen die Ionen mit einem Stoßgas (z.B. Helium). Die dadurch erzwungene Energieübertragung kann zur unimolekularen Dissoziation der Analyt-Ionen führen. Fragment-Ionen, die in dieser Gasphasenreaktion entstehen, können erneut massenselektiert und fragmentiert werden (MS ${ }^{n}$-Experimente). So können CID-Experimente im Allgemeinen nicht nur einen wichtigen Beitrag für die Identifizierung unbekannter Analyt-Ionen liefern, sondern ermöglichen auch die Untersuchung der intrinsischen unimolekularen Reaktivität ausgewählter lonen in der Gasphase, in der chemische Gleichgewichte und Solvenseffekte keinen störenden Einfluss ausüben. Zusätzlich ist es möglich, thermodynamische Größen und kinetische Informationen mithilfe von CID-Experimenten direkt zu bestimmen.

Neben der unimolekularen kann insbesondere die bimolekulare Gasphasenreaktivität selektierter lonen gegenüber ausgewählten, neutralen Reaktionspartnern von großem Interesse für mechanistische Studien sein. Um sogenannte lonen-Molekül-Reaktionen (IMR) unter kontrollierten Bedingungen zu untersuchen, wurden in der Vergangenheit bereits verschiedenste massenspektrometrische Techniken angewandt. ${ }^{[60]}$ Die dabei gängigsten Analysegeräte und -methoden waren Fourier-Transformation-lonen-Cyclotron-Massenspektrometer (FT-ICR MS) und selected-ion flow-tubes (SIFT). Zusätzlich fanden bereits Quadrupol-Ionenfallen-Massenspektrometer zur Untersuchung bimolekularer Gasphasenreaktivitäten Anwendung. Um diese im Detail analysieren zu können, bestehen im Wesentlichen zwei Möglichkeiten. ${ }^{\left[{ }^{61]}\right.}$ Eine Option ist das Installieren eines Ventils an das Hochvakuum-System des Massenspektrometers, über welches der neutrale Reaktionspartner mit einer konstanten Flussrate direkt eingebracht wird. Problematisch an dieser Methode ist der Partialdruck des neutralen Reaktantgases, dessen Bestimmung abhängig vom verwendeten Gerätetyp sehr schwierig sein kann. Als Alternative kann das Gasgemisch aus Reaktantgas und Helium extern in wohldefinierten Partialdrücken der einzelnen Gase erstellt und anschließend mit einer konstanten Flussrate in das Massenspektrometer transferiert werden. 


\subsection{Elektrische Konduktometrie}

\subsubsection{Funktionsweise}

Durch die Anwendung elektrischer Konduktometrie ist ein direkter Zugang zur spezifischen elektrischen Leitfähigkeit $\kappa / \mathrm{S} \mathrm{cm}^{-1}$ von Probenlösungen möglich. $\kappa$ ist dabei ein Maß für alle Ionen, die in einer Lösung vorliegen, und ist von stoffspezifischen Parametern der Kationen und Anionen geprägt (Gleichung 2.3-1). ${ }^{[62]}$

$$
\kappa=F \cdot c \cdot\left(v^{+} Z^{+} u^{+}+v^{-} Z^{-} u^{-}\right)
$$

Stöchiometrische Faktoren $\left(v^{+-}\right)$, Ladungszahlen $\left(z^{+-}\right)$und die elektrische Beweglichkeit der Ionen $\left(u^{+-}\right)$haben einen entscheidenden Einfluss auf die Leitfähigkeit $\kappa$. Die FaradayKonstante $F$ ist zur vollständigen Beschreibung von $\kappa$ nötig und entspricht der Ladung von einem Mol einfach geladener lonen. ${ }^{[63]}$ Daneben ist die spezifische elektrische Leittähigkeit stark von der Stoffmengenkonzentration $c$ der lonen in Lösung abhängig. So verhält sich $\kappa$ für stark verdünnte Lösungen annähernd proportional zur Stoffmengenkonzentration. Mit steigender Konzentration ist ein nicht-lineares Verhalten zu beobachten. ${ }^{[62,63]}$ Grund hierfür ist bei schwachen Elektrolyten eine unvollständige Dissoziation. Bei starken Elektrolyten greift die Debye-Hückel-Onsager-Theorie. In diesem Fall kann eine gegenseitige Abstoßung der lonen die Nicht-Linearität erklären. Zusätzlich ist die spezifische elektrische Leittähigkeit $\kappa$ stark von der Temperatur abhängig. Diese wirkt sich auf die Beweglichkeit der lonen $\left(u^{+-}\right)$in Lösung aus (Gleichung 2.3-2). ${ }^{[62]}$

$$
u^{+/}=\frac{z^{+/} \cdot e}{6 \pi \cdot r^{+-} \cdot \eta}
$$

Die Temperaturabhängigkeit der Beweglichkeit der lonen kommt durch die Viskosität $\eta$ des Lösungsmittels zustande, die direkt von der Temperatur beeinflusst wird. Zudem kann sich der Solvatisierungsgrad und damit der effektive Radius der Kationen und Anionen $\left(r^{+/}\right)$mit der Temperatur verändern.

Die elektrische Konduktometrie ist eine ideale Ergänzung zur ESI-Massenspektrometrie und ermöglicht im Gegensatz zu dieser die Quantifizierung von Ionen in Lösung. So konnte in der Vergangenheit bereits gezeigt werden, dass die elektrische Leitfähigkeit $\kappa$ zusätzliche Informationen lieferte, um Metallat-Komplexe in Lösung zu quantifizieren und damit zu charakterisieren. ${ }^{[55 b, 55 d, 64]}$ Ohne zusätzliche Analysemethoden und Vergleichsmöglichkeiten jedoch ist die elektrische Konduktometrie nur schwer zu interpretieren und wenig aussagekräftig. 


\subsection{Mößbauer-Spektroskopie}

\subsubsection{Funktionsweise}

Die Mößbauer-Spektroskopie ist eine Analysemethode von Kernzuständen, die sehr sensitiv auf magnetische und elektronische Veränderungen reagiert. ${ }^{[65]}$ Grundlage dieser Methode ist der Mößbauer-Effekt ( $\rightarrow$ Abbildung 7, links), für dessen Entdeckung Rudolph Mößbauer 1961 mit dem Nobelpreis für Physik ausgezeichnet wurde. ${ }^{[66]}$ Der Mößbauer-Effekt beschreibt den Zerfall eines Atomkerns unter Emission von $\gamma$-Strahlung. ${ }^{[67]}$ Ein zweiter identischer Atomkern kann die Energie der $\gamma$-Strahlung absorbieren und wird dadurch in einen energetisch angeregten Zustand angehoben. Allerdings kann die $\gamma$-Strahlung nur dann absorbiert werden, wenn dieser Prozess rückstoßfrei erfolgt und die Energie der emittierten $\gamma$-Strahlung der Anregungsenergie des zweiten Atomkerns entspricht. Diese Bedingungen sind nur im festen Aggregatzustand erfüllt, weshalb die Analyse mittels Mößbauer-Spektroskopie nur von Feststoffen und gefrorenen Lösungen möglich ist. Zudem sollte die Mößbauer-Spektroskopie bei tiefen Temperaturen durchgeführt werden, da sich so die Wahrscheinlichkeit von rückstoßfreien Absorptionsprozessen stark erhöht. Am gängigsten wird die MößbauerSpektroskopie zur Detektion von ${ }^{57} \mathrm{Fe}$-Kernen verwendet. ${ }^{[68]}$ Prinzipiell lassen sich aber auch weitere Kerne mit dieser Methode nachweisen, vor allem dann, wenn passende und praktikable $\gamma$-Strahlungsquellen verfügbar sind. So findet die Mößbauer-Spektroskopie auch

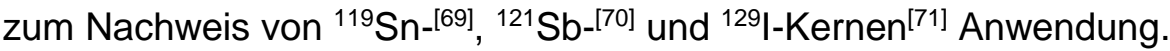

In der ${ }^{57} \mathrm{Fe}$-Mößbauer-Spektroskopie wird eine radioaktive Cobaltquelle $\left({ }_{27}^{57} \mathrm{Co}\right)$ genutzt, die über Elektroneneinfang hochangeregte Eisenkerne $\left({ }_{26}^{57} \mathrm{Fe}^{*}\right)$ erzeugt ( $\rightarrow$ Abbildung 7 , rechts). ${ }^{[2]}$ Von diesem Zustand aus sind mehrere Übergänge in den Grundzustand möglich. Dabei ist der für die Mößbauer-Spektroskopie relevante Übergang ( $E=14.4 \mathrm{keV}, \tau=140 \mathrm{~ns}$ ) von seiner Energie und Halbwertszeit her besonders gut geeignet. Die Erzeugung energetisch angeregter Eisenkerne des Isotops 57 in Kombination mit der Tatsache, dass der Mößbauer-Effekt auf zwei identischen Atomkernen basiert, sind der Grund dafür, dass nur ${ }^{57} \mathrm{Fe}-K e r n e ~ M o ̈ ß b a u e r-$ aktiv sind. Hieraus ergibt sich, dass insbesondere Proben gefrorener Lösungen relativ hoch konzentriert sein müssen (natürliche Häufigkeit ${ }^{57} \mathrm{Fe} \approx 2.19 \%$ ) ${ }^{[73]}$ oder alternativ aus angereichertem ${ }^{57} \mathrm{Fe}$-Verbindungen synthetisiert werden müssen, um qualitativ hochwertige Ergebnisse zu liefern. 


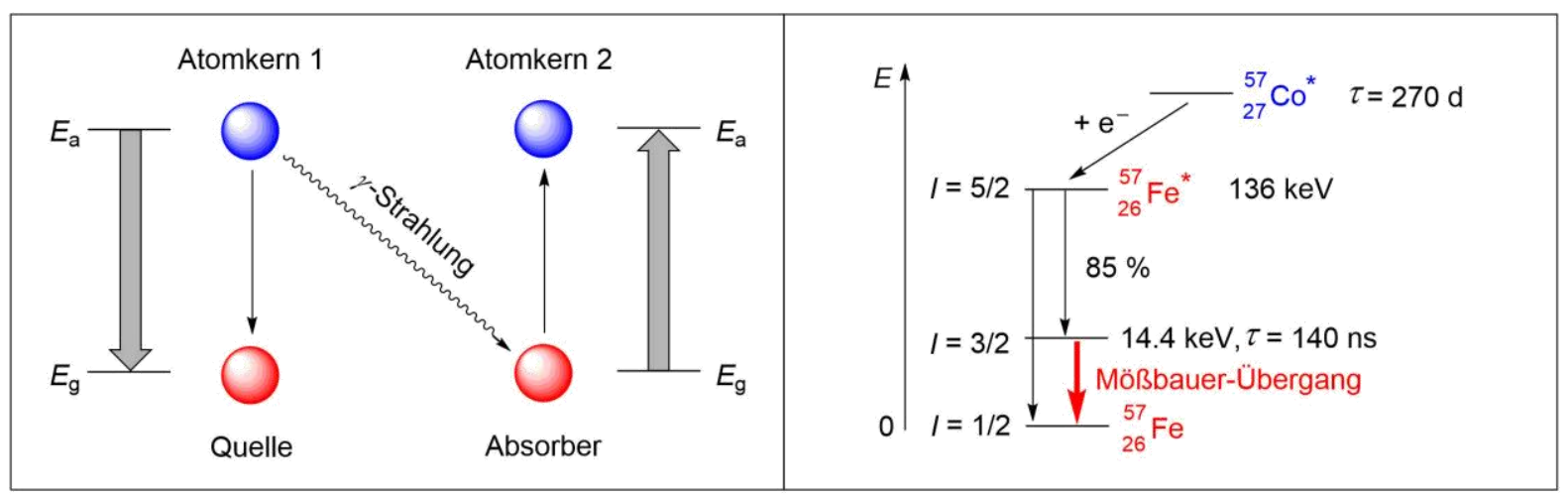

Abbildung 7. Links: Prinzip des Mößbauer-Effekts. Rechts: Relevante Kerne und energetische Übergänge bei der ${ }^{57} \mathrm{Fe}-\mathrm{Mößbauer-Spektroskopie.}{ }^{[67,72]}$

Zur Variation der Energie der $\gamma$-Strahlung macht sich die Mößbauer-Spektroskopie den Doppler-Effekt zunutze. ${ }^{[67]}$ In der Praxis bedeutet dies, dass die radioaktive Quelle in Relation zum Absorber mechanisch bewegt wird. Abschließend wird der nicht resonant-absorbierte Teil der $\gamma$-Strahlung in Abhängigkeit von der Geschwindigkeit der radioaktiven Quelle in Relation zum Absorber detektiert. ${ }^{[67,72]}$ Im daraus resultierenden Spektrum wird die relative Transmission der $\gamma$-Strahlung gegen die Geschwindigkeit $\left(\mathrm{mm} \mathrm{s}^{-1}\right)$ aufgetragen. Im Wesentlichen lassen sich aus einem Mößbauer-Spektrum drei charakteristische Werte entnehmen: Die Isomerieverschiebung $\delta$ und die Quadrupolaufspaltung $\Delta E_{\mathrm{Q}}$ ( $\rightarrow$ Abbildung 8) sowie, falls auftretend, die magnetische Aufspaltung $\Delta E_{\mathrm{M}}$.

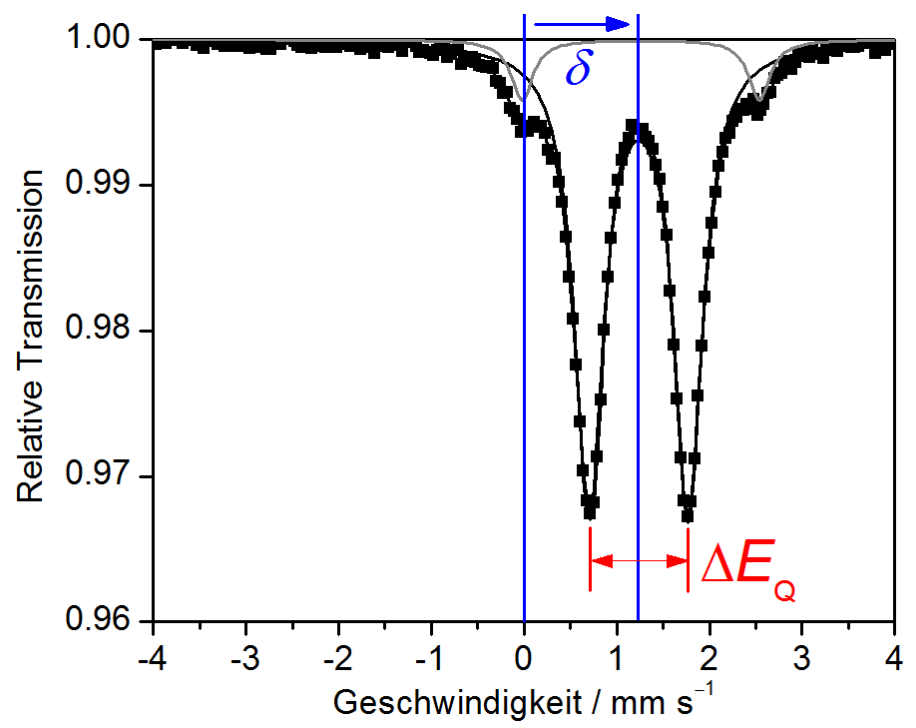

Abbildung 8. Beispiel eines typischen Mößbauer-Spektrums $(T=80 \mathrm{~K})$. Zu sehen ist das Mößbauer-Dublett von $\mathrm{FeCl}_{2}$. Die Mößbauer-Parameter $\delta=1.24 \mathrm{~mm} \mathrm{~s}^{-1}$ und $\Delta E_{\mathrm{Q}}=1.07 \mathrm{~mm} \mathrm{~s}^{-1}$ sind in ihrer Kombination charakteristisch für eine Eisen(II)-Spezies mit high-spin-Charakter. In Spuren ist ein zweites Signal zu erkennen, das auf die Anwesenheit eines Hydrolyseproduktes von $\mathrm{FeCl}_{2}$ hindeutet. 
Die Isomerieverschiebung $\delta$, entstanden aus der Wechselwirkung von Kernen mit der elektronischen Umgebung, ermöglicht Rückschlüsse auf die Oxidationszahl des untersuchten Kerns sowie auf die Elektronegativität vorhandener Liganden. ${ }^{[2]}$ Auch die Quadrupolaufspaltung $\Delta E_{Q}$, die auf einer Wechselwirkung des Quadrupolmomentes mit einem inhomogenen elektrischen Feld um den Kern beruht, ist charakteristisch für spezifische Oxidationsstufen der untersuchten Kerne. Zusätzlich bietet die Quadrupolaufspaltung die Möglichkeit, Informationen über den Spinzustand untersuchter Proben zu erhalten. Folglich können somit auch Aussagen über die Geometrie untersuchter Verbindungen getroffen werden. Die magnetische Aufspaltung $\Delta E_{M}$ bietet Rückschlüsse auf die magnetischen Eigenschaften einer Probe. Da die im Rahmen dieser Arbeit untersuchten Proben keinen Zugang zu $\Delta E_{\mathrm{M}}$ boten, soll auf diesen Mößbauer-Parameter im Folgenden nicht näher eingegangen werden.

Im Gegensatz zur ESI-Massenspektrometrie ermöglicht die Mößbauer-Spektroskopie die Detektion sämtlicher Eisen-Spezies einer Probe, unabhängig davon, ob diese geladen oder neutral in Lösung vorliegen. Ohne ergänzende Analysemethode reicht die MößbauerSpektroskopie allein zumeist nicht aus, um Eisenverbindungen hinsichtlich ihrer Struktur und Bindungseigenschaften vollständig $\mathrm{zu}$ charakterisieren. In Kombination mit ESIMassenspektrometrie jedoch bietet sie eine sehr gute und umfassende Analysemöglichkeit von eisenhaltigen Proben. 


\section{Zielsetzung}

Eisen-katalysierte Kreuzkupplungsreaktionen zeigen ein großes Potential als Alternative zu gängigen Kreuzkupplungsreaktionen mit Palladium- oder Nickel-Katalysatoren, sind nach aktuellem Stand aber mechanistisch nur unzureichend verstanden. Durch die in dieser Arbeit gesammelten Erkenntnisse soll ein breiteres mechanistisches Verständnis von Eisenkatalysierten Kreuzkupplungsreaktionen geschaffen werden. Dies soll dazu beitragen, Eisenkatalysierte Kreuzkupplungen durch gezielte Optimierung zu einer noch wertvolleren und effizienteren Synthesemethode insbesondere in der Industrie zu entwickeln.

Ein erstes Ziel dieser Arbeit war der Nachweis Eisen-organischer Spezies, speziell der Organoferrate, mit ESI-Massenspektrometrie, Mößbauer-Spektroskopie und elektrischer Konduktometrie sowie die Einstufung dieser als mögliche Reaktionsintermediate Eisenkatalysierter Kreuzkupplungsreaktionen. Dabei sollen die einzelnen Elementarreaktionen entsprechend der Abfolge des im allgemein angenommenen Katalysezyklus bestehend aus Transmetallierung, Additionsprozess eines organischen Substrates und reduktiver Eliminierung im Detail untersucht und abschließend in einem Katalysezyklus zusammengeführt werden. Die Transmetallierung soll in Abhängigkeit vom EisenPräkatalysator, vom metallorganischen Transmetallierungsreagenz und von der Anwesenheit verschiedenster Additive und Liganden näher beleuchtet werden. Die dabei entstandenen Eisen-Spezies sollen in ihrer Reaktivität gegenüber organischen (Pseudo)-Halogeniden in Lösung charakterisiert werden. Um die spezifische Reaktivität wohldefinierter Organoferrate gegenüber diesen Substraten näher zu beschreiben, soll eine Apparatur entwickelt werden, die durch Kopplung an ein Quadrupol-Ionenfallen-Massenspektrometer die Untersuchung bimolekularer Gasphasenreaktivitäten ermöglicht. Zusätzlich sollen unimolekulare Fragmentierungsreaktionen in der Gasphase wichtige Informationen über die reduktive Eliminierung als entscheidenden Schritt innerhalb des Mechanismus Eisen-katalysierter Kreuzkupplungsreaktionen liefern. 


\section{Ergebnisse und Diskussion}

\subsection{Transmetallierung}

Es gilt als gesicherte Tatsache, dass zu Beginn des Katalysezyklus Eisen-katalysierter Kumada-Kreuzkupplungsreaktionen über Transmetallierung (und Reduktion) eines EisenPräkatalysators durch Grignard-Reagenzien Eisen-organische Spezies entstehen. ${ }^{[38]}$ In der Folge können diese mit einem organischen Substrat in noch ungeklärter Weise reagieren, um abschließend das Kreuzkupplungsprodukt freizusetzen. Um den zugrunde liegenden Mechanismus im Detail zu verstehen, ist es zu Beginn erforderlich, die über Transmetallierung (und Reduktion) entstandenen Eisen-Spezies in Abwesenheit des Kupplungspartners zu identifizieren und zu charakterisieren.

\subsubsection{Transmetallierung in Abwesenheit von Additiven und Liganden}

\subsubsection{Transmetallierung von FeX $2 / F_{3} X_{3}$ mit PhMgX}

$\mathrm{Fe}(\mathrm{acac})_{3}$ zeichnet sich durch seine besonders hohe Unempfindlichkeit gegenüber Hydrolyseund Oxidationsreaktionen aus. Aus diesem Grund erfolgten erste Experimente zur detaillierten Untersuchung der Transmetallierung gängiger Eisen-Präkatalysatoren mit GrignardReagenzien ausgehend von $\mathrm{Fe}(\mathrm{acac})_{3}$.

Die ESI-massenspektrometrische Untersuchung von Probenlösungen von $\mathrm{Fe}(\mathrm{acac})_{3}$ in $\mathrm{THF}$ nach Zugabe von PhMgCl (1.0-3.0 Äq.) zeigte im negativen lonenmodus die Reduktion von $\mathrm{Fe}(\mathrm{III}) \mathrm{zu} \mathrm{Fe}(\mathrm{II})$. Wurden den Probenlösungen insgesamt vier Äquivalente an $\mathrm{PhMgCl}$ zugesetzt, konnte die Bildung der mononuklearen Phenylferrate $\mathrm{Ph}_{3} \mathrm{Fe}(\mathrm{II})^{-}$und $\mathrm{Ph}_{4} \mathrm{Fe}(\mathrm{III})^{-}$ beobachtet werden ( $\rightarrow$ Abbildung 9 und 10). Die Identifizierung dieser beiden Spezies erfolgte auf Grundlage der Isotopenmuster und der exakten Massen der entsprechenden Peaks. Das Isotopenmuster von $\mathrm{Ph}_{4} \mathrm{Fe}(\mathrm{III})^{-}$soll dabei beispielhaft für alle mononuklearen Organoferrate stehen. Auf die Darstellung zusätzlicher Isotopenmuster einkerniger Organoferrate wird im weiteren Verlauf dieser Arbeit verzichtet. Zusätzlich zeigte sich im resultierenden Massenspektrum das vierkernige Aggregat $\mathrm{Ph}_{7} \mathrm{Fe}_{4}{ }^{-}$in geringer, aber stabiler Signalintensität $(\rightarrow$ Abbildung 11). Dieses beinhaltete Eisen in einer durchschnittlichen Oxidationsstufe von 1.5 . 


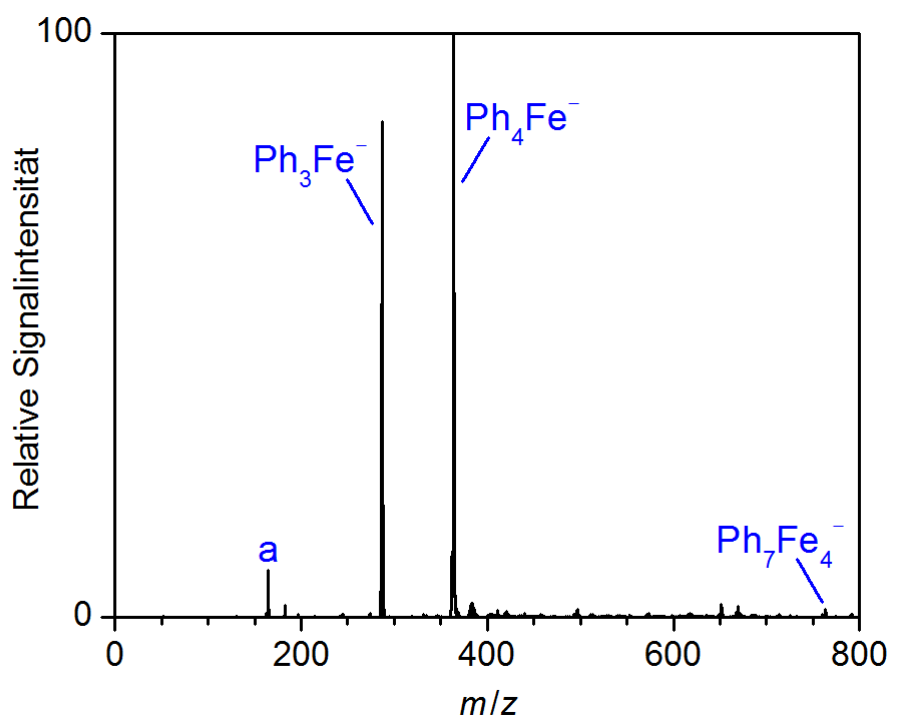

Abbildung 9. ESI-Massenspektrum (negativer lonenmodus) der Produkte aus der Reaktion von $\mathrm{Fe}(\mathrm{acac})_{3}(20 \mathrm{~mm})$ und $\mathrm{PhMgCl}$ (4.0 Äq.) in $\mathrm{THF} ; \mathrm{a}=\left[\mathrm{Ph}, \mathrm{Fe}, \mathrm{O}_{2}\right]^{-}$.

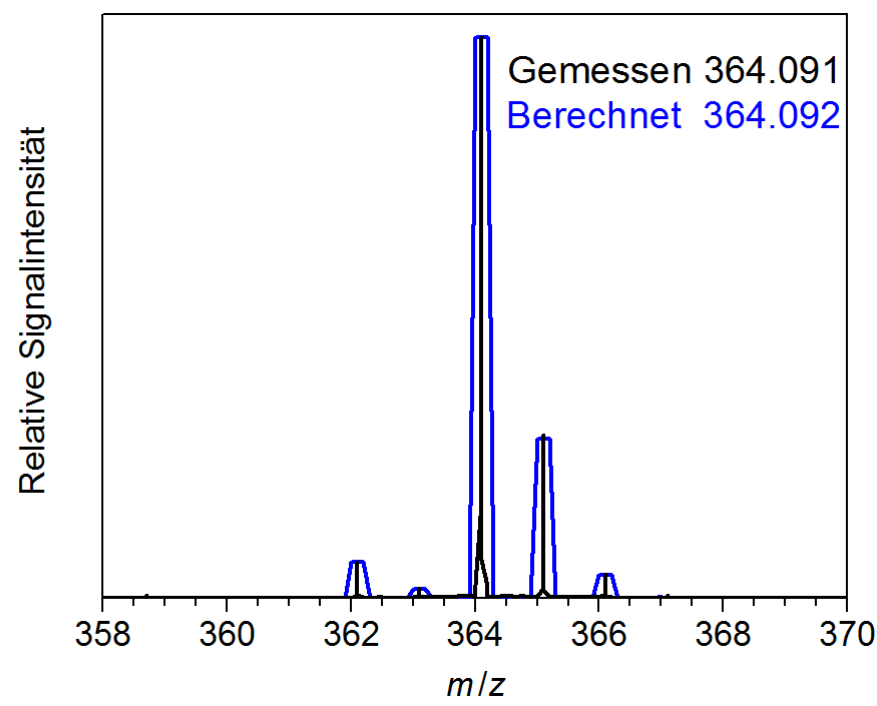

Abbildung 10. Vergleich des gemessenen (schwarz) und des simulierten (blau) Isotopenmusters von $\mathrm{Ph}_{4} \mathrm{Fe}^{-}$. 


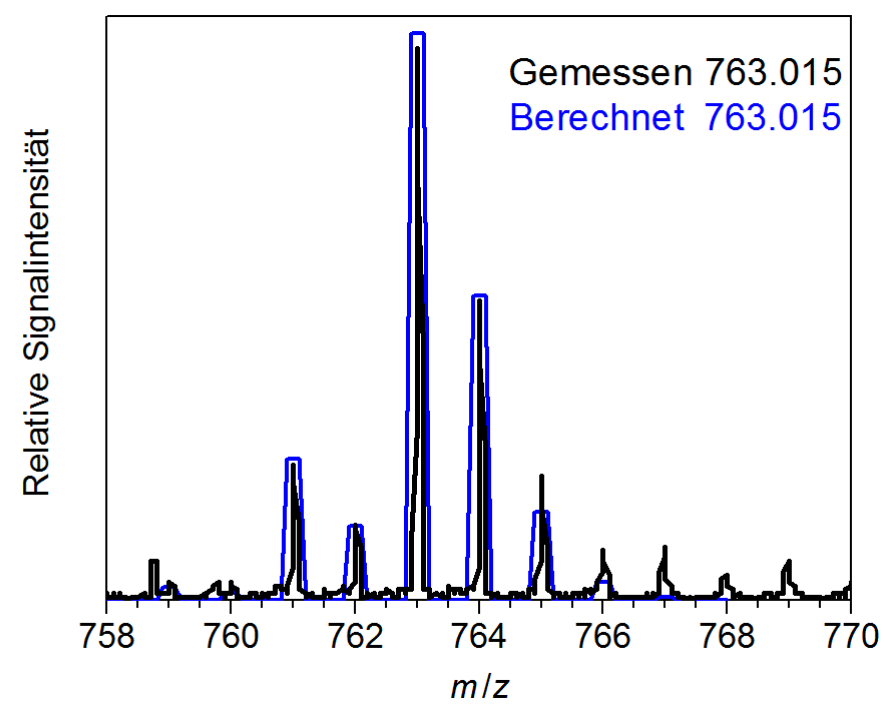

Abbildung 11. Vergleich des gemessenen (schwarz) und des simulierten (blau) Isotopenmusters von $\mathrm{Ph}_{7} \mathrm{Fe}_{4}{ }^{-}$.

Die Bildung mehrkerniger Phenylferrate findet Bestätigung in der Literatur. Schoch et al. konnten mittels Röntgenabsorptionsspektroskopie nachweisen, dass Reaktionslösungen von $\mathrm{Fe}(\mathrm{acac})_{3}$ mit $\mathrm{PhMgCl}$ (3.0 oder 4.0 Äq.) in Anwesenheit von NMP zur Bildung von drei- und vierkernigen Aggregaten führte. ${ }^{[35 e]}$ Im kleinen Massenbereich wurde zudem eine Spezies der allgemeinen Zusammensetzung $\left[\mathrm{Ph}, \mathrm{Fe}, \mathrm{O}_{2}\right]^{-}$als Produkt einer bimolekularen Reaktion mit Sauerstoff beobachtet. Dieser war in Spuren im Hochvakuumsystem des Massenspektrometers vorhanden.

Die mononuklearen Phenylferrate $\mathrm{Ph}_{3} \mathrm{Fe}(\mathrm{II})^{-}$und $\mathrm{Ph}_{4} \mathrm{Fe}(\mathrm{III})^{-}$unterschieden sich deutlich in ihrer Stabilität. Kinetische Messungen, durchgeführt mittels ESI-Massenspektrometrie, zeigten, dass die anionische Fe(II)-Spezies mit einer Halbwertszeit von $t_{1 / 2}=50 \pm 15 \mathrm{~s}$ deutlich instabiler war als $\mathrm{Ph}_{4} \mathrm{Fe}(\mathrm{III})^{-}$, die im gesamten Messzeitraum von $t=30$ min stabil erschien $\left(\rightarrow\right.$ Abbildung 12). Auffällig war, dass mit dem Zerfall von $\mathrm{Ph}_{3} \mathrm{Fe}(\mathrm{II})^{-}$die Bildung der $\mathrm{Fe}(\mathrm{III})^{-}$ Spezies einherging.

Die analoge Transmetallierungsreaktion mit $\mathrm{PhMgBr}$ anstelle von $\mathrm{PhMgCl}$ resultierte erneut in der Bildung von $\mathrm{Ph}_{3} \mathrm{Fe}^{-}, \mathrm{Ph}_{4} \mathrm{Fe}^{-}, \mathrm{Ph}_{7} \mathrm{Fe}_{4}{ }^{-}$und $\left[\mathrm{Ph}, \mathrm{Fe}, \mathrm{O}_{2}\right]^{-}$, wobei eine höhere Gesamtintensität der lonen beobachtet werden konnte. Auch die Transmetallierung mit zehn Äquivalenten an $\mathrm{PhMgCl}$ führte zu einem vergleichbaren Ergebnis. 


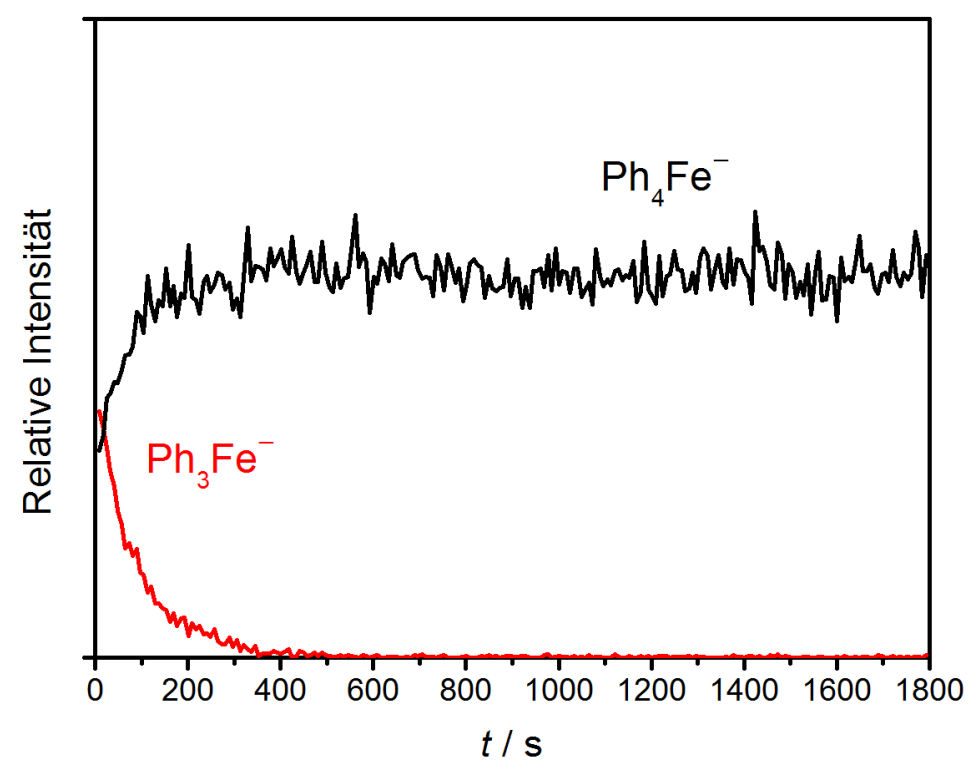

Abbildung 12. ESI-massenspektrometrisch detektierte, zeitliche Entwicklung der relativen Intensitäten der lonen $\mathrm{Ph}_{3} \mathrm{Fe}^{-}$und $\mathrm{Ph}_{4} \mathrm{Fe}^{-}$, entstanden durch Reaktion von $\mathrm{Fe}(\mathrm{acac})_{3}(20 \mathrm{~mm})$ mit $\mathrm{PhMgCl}$ (4.0 Äq.) in THF.

Neben $\mathrm{Fe}(\mathrm{acac})_{3}$ wurden mit $\mathrm{FeCl}_{3}, \mathrm{FeCl}_{2}$ und $\mathrm{Fe}(\mathrm{OAc})_{2}$ weitere gängige EisenPräkatalysatoren mit $\mathrm{PhMgCl}$ (4.0 Äq.) umgesetzt. In allen Fällen dominierten die bereits bekannten Phenylferrate $\mathrm{Ph}_{3} \mathrm{Fe}(\mathrm{II})^{-}$und $\mathrm{Ph}_{4} \mathrm{Fe}(\mathrm{III})^{-}$die ESI-Massenspektren im negativen Ionenmodus. Auffallend war, dass die Transmetallierung von $\mathrm{FeCl}_{2}$ zur Ausbildung der heterobimetallischen Spezies $\mathrm{Ph}_{6} \mathrm{MgFe}(\mathrm{III})^{-}$und $\mathrm{Ph}_{5} \mathrm{MgFe}(\mathrm{III}) \mathrm{Cl}^{-}$führte. Das erstgenannte Anion konnte auch nach Transmetallierung von $\mathrm{Fe}(\mathrm{OAc})_{2}$ mit $\mathrm{PhMgCl}$ (4.0 Äq.) beobachtet werden. Die Bildung solcher heterobimetallischen Phenylferrate deutete auf eine direkte Interaktion der Ferrat-Komplexe mit Magnesium hin.

Als komplementäre Methode zu ESI-Massenspektrometrie wurden Leitfähigkeitsmessungen durchgeführt. Diese Untersuchungen ermöglichten quantitative Interpretationen, ergänzend zu den qualitativen Ergebnissen aus massenspektrometrischen Analysen. So zeigte sich, dass die Phenylferrate in Kombination mit ihren Mg-haltigen Gegenionen in THF eine signifikante und über einen Zeitraum von $t=30 \mathrm{~min}$ bei $273 \mathrm{~K}$ nahezu konstante spezifische elektrische Leitfähigkeit $\kappa$ aufwiesen. ( $\rightarrow$ Abbildung 13). Dies lieferte einen weiteren Hinweis darauf, dass Phenylferrate in Lösung eine entscheidende Rolle spielen können. 


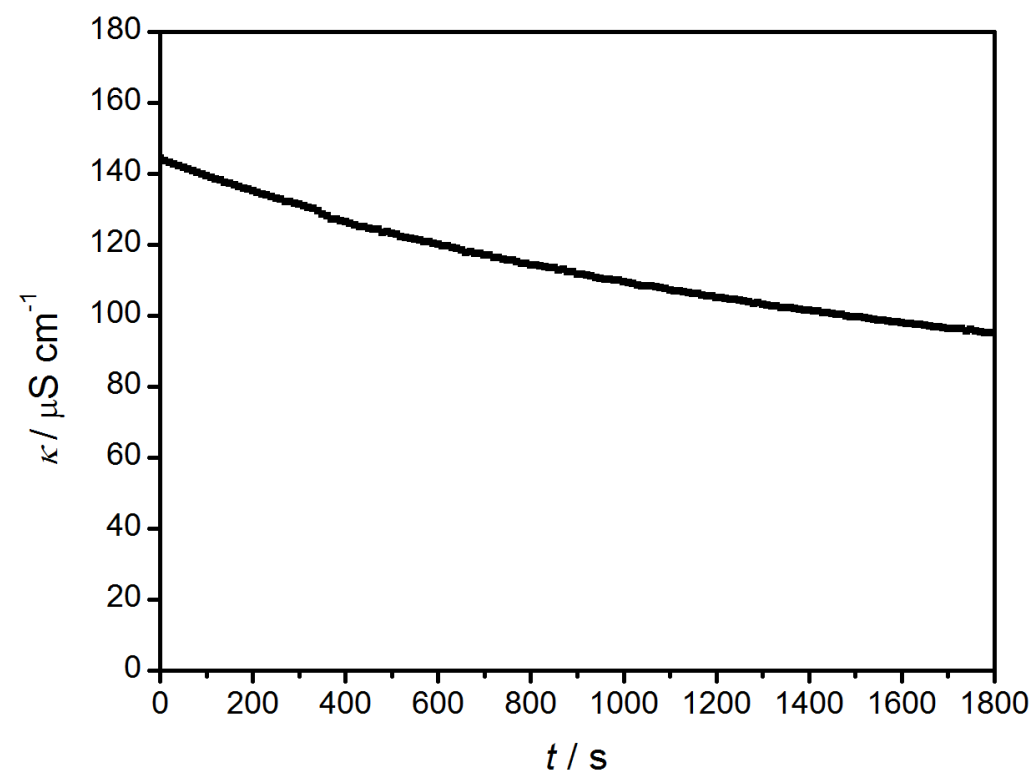

Abbildung 13. Zeitliche Entwicklung der spezifischen elektrischen Leitfähigkeit $\kappa$ einer Reaktionslösung von $\mathrm{Fe}(\mathrm{acac})_{3}(50 \mathrm{~mm})$ und $\mathrm{PhMgCl}$ (4.0 Äq.) in THF bei $T=273 \mathrm{~K}$.

Zum Vergleich wurden die elektrischen Leitfähigkeiten $\kappa$ der aus Transmetallierung weiterer Eisenvorläufer mit $\mathrm{PhMgCl}$ (4.0 Äq.) resultierenden lonen experimentell bestimmt $(\rightarrow$ Tabelle 3). Dabei fiel auf, dass die ursprüngliche Oxidationsstufe von Eisen keinen Einfluss auf die elektrische Leitfähigkeit hatte. Vielmehr war diese durch die Natur der Gegenionen X$(\mathrm{X}=\mathrm{acac}, \mathrm{Cl}, \mathrm{OAc})$ entscheidend geprägt.

Tabelle 3. Spezifische elektrische Leitfähigkeit $\kappa /\left(\mu \mathrm{S} \mathrm{cm}^{-1}\right)$ von Phenylferraten und ihren Mg-haltigen Gegenionen gebildet durch Transmetallierung verschiedener Eisenvorläufer (50 mM) mit PhMgCl (4.0 Äq.) in THF bei $T=273 \mathrm{~K}$.

\begin{tabular}{ll}
\hline \multicolumn{1}{c}{ Probenlösung in THF } & $\boldsymbol{\kappa} / \boldsymbol{\mu} \mathbf{~ c m}^{-1}$ \\
\hline $\mathrm{Fe}(\mathrm{acac})_{3}+4 \mathrm{PhMgCl}$ & $99 \pm 16$ \\
$\mathrm{FeCl}_{3}+4 \mathrm{PhMgCl}$ & $59 \pm 1$ \\
$\mathrm{FeCl}_{2}+4 \mathrm{PhMgCl}$ & $57 \pm 3$ \\
$\mathrm{Fe}(\mathrm{OAc})_{2}+4 \mathrm{PhMgCl}$ & $98 \pm 6$ \\
\hline
\end{tabular}

Um die Präsenz der Phenylferrate in Lösung zu bestätigen und auszuschließen, dass diese nicht nur als Artefakte des ESI-Prozesses entstanden waren, wurde zusätzlich ein MößbauerExperiment durchgeführt. Dabei war ${ }^{57} \mathrm{FeCl}_{2}$ der Eisenvorläufer der Wahl, da dieser aus ${ }^{57} \mathrm{Fe}-$ Pulver besonders einfach zugänglich war. ${ }^{[74]}$ Das resultierende Mößbauer-Spektrum einer eingefrorenen Probenlösung von ${ }^{57} \mathrm{FeCl}_{2}$ mit $\mathrm{PhMgCl}$ (4.0 Äq.) in THF zeigte ein breites und 
asymmetrisches Dublett bei einer Isomerieverschiebung von $\delta=0.50 \mathrm{~mm} \mathrm{~s}^{-1}$ und einer Quadrupolaufspaltung von $\Delta E_{\mathrm{Q}}=1.06 \mathrm{~mm} \mathrm{~s}^{-1}$ ( $\rightarrow$ Abbildung 14).

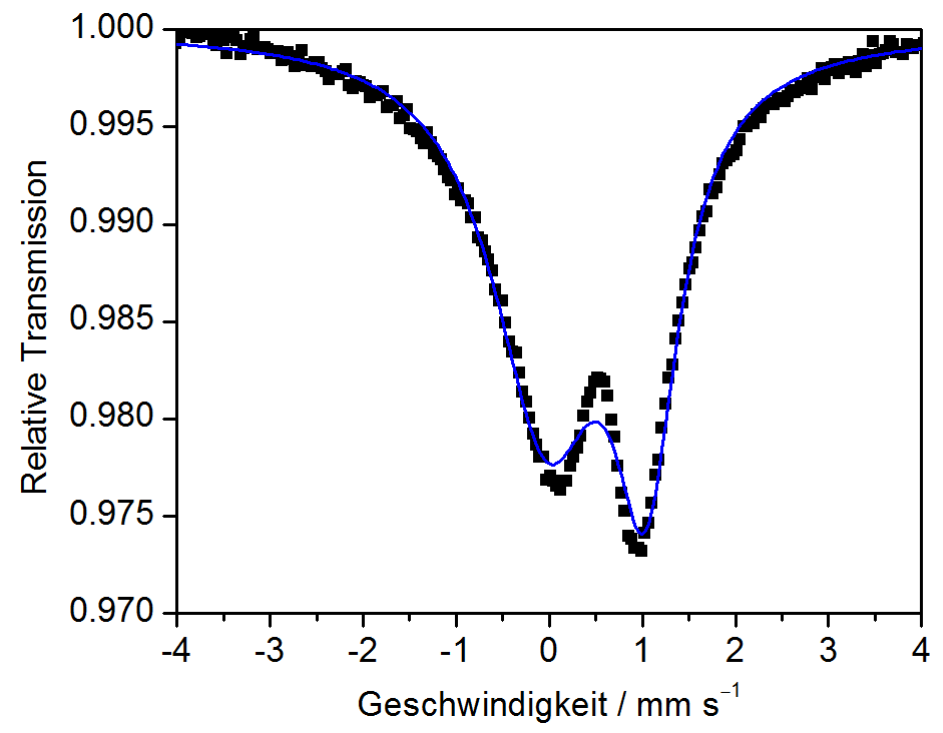

Abbildung 14. Mößbauer-Spektrum einer gefrorenen Lösung der Produkte aus der Reaktion von ${ }^{57} \mathrm{FeCl}_{2}$ mit $\mathrm{PhMgCl}\left(4.0\right.$ Äq.) in THF $(T=80 \mathrm{~K}) ; \delta=0.50 \mathrm{~mm} \mathrm{~s}^{-1}, \Delta E_{\mathrm{Q}}=1.06 \mathrm{~mm} \mathrm{~s}^{-1}$, rel. Intensität $=100 \%$.

Das Mößbauer-Signal deutete auf die Gegenwart von mindestens einer Fe(III)-Spezies in Lösung hin. Aufgrund komplexer magnetischer Effekte und deren Auswirkung auf die Symmetrie und Breite des Mößbauer-Dubletts war eine genauere Angabe über die Anzahl an $\mathrm{Fe}(\mathrm{III})$-Spezies sowie deren Spinzustände nicht möglich. Es konnte kein Hinweis auf eine gleichzeitig vorliegende $\mathrm{Fe}(\mathrm{II})$-Spezies in Lösung gefunden werden. Dies ist nicht verwunderlich, da $\mathrm{Ph}_{3} \mathrm{Fe}(\mathrm{II})^{-}$mit einer Halbwertszeit von $t_{1 / 2}=50 \pm 15 \mathrm{~s}$ vermutlich bereits während der Präparation der Mößbauer-Messzelle zerfiel.

Massenspektren im positiven lonenmodus der Probenlösungen von $\mathrm{Fe}(\mathrm{acac})_{3}$ mit $n \mathrm{PhMgCl}$ $(n=1,2)$ wurden von Mg-haltigen Spezies des Typs $\operatorname{Mg}_{m}(\mathrm{acac})_{2 m-1}(\mathrm{THF})_{n}{ }^{+}(m=1-3, n=0-2)$ dominiert. Nach Zugabe weiterer Äquivalente an $\mathrm{PhMgCl}$ konnten zusätzlich $\mathrm{Mg}_{3}(\mathrm{acac})_{4} \mathrm{Cl}(\mathrm{THF})^{+}$und $\mathrm{Mg}_{4}(\mathrm{acac})_{6} \mathrm{Cl}^{+}$detektiert werden. Bei einem hohen Überschuss an Grignard-Reagenz (> 4 Äq.) waren ausschließlich Kationen des $\operatorname{Typs} \mathrm{Mg}_{3} \mathrm{Cl}_{3}(\mathrm{OMe})_{2}(\mathrm{THF})_{n}{ }^{+}$ $(n=5,6)$ vorhanden. Diese Mg-Spezies waren auf eine Reaktion mit Spuren von Methanol zurückzuführen, welches im Hochvakuumsystem des Massenspektrometers nach Reinigung desselben verblieb. ${ }^{[75]}$ Im weiteren Verlauf dieser Arbeit wird auf eine detaillierte Beschreibung von ESI-Massenspektren im positiven lonenmodus verzichtet, sollten diese qualitativ den eben beschriebenen Massenspektren ähneln. Treten markante Veränderungen auf, wird auf diese explizit hingewiesen. 


\subsubsection{Transmetallierung von $\mathrm{Fe}(\mathrm{acac})_{3}$ mit $\mathrm{ArMgBr}$}

In Kapitel 4.1.1.1 konnte erfolgreich nachgewiesen werden, dass die Natur des Eisenvorläufers keinen entscheidenden qualitativen Einfluss auf die Bildung von Phenylferraten hat. Aus diesem Grund wurde für alle weiteren massenspektrometrischen Experimente hauptsächlich auf $\mathrm{Fe}(\mathrm{acac})_{3}$ als besonders einfach $\mathrm{zu}$ handhabenden Eisenvorläufer zurückgegriffen. Wurden andere Eisen-Präkatalysatoren verwendet, wird im weiteren Verlauf dieser Arbeit explizit darauf hingewiesen.

Das ESI-Massenspektrum einer Probenlösung von $\mathrm{Fe}(\mathrm{acac})_{3}$ mit $\mathrm{MesMgBr}$ (4.0 Äq.) im negativen lonenmodus wurde dominiert von $\mathrm{Mes}_{3} \mathrm{Fe}(\mathrm{II})^{-}$bei $\mathrm{m} / z$ 413. ( $\rightarrow$ Abbildung 15 ). Dieses Anion zeichnete sich durch seine besonders hohe Signalintensität aus, die auch über vergleichsweise lange Messzeiten $(t>1 \mathrm{~h})$ stabil war. Zusätzlich konnten in geringer Signalintensität Spuren von $\mathrm{Mes}_{2} \mathrm{Fe}(\mathrm{II}) \mathrm{Br}^{-}$detektiert werden. Eine signifikante elektrische Leitfähigkeit von $\kappa=339 \pm 6 \mu \mathrm{S} \mathrm{cm}{ }^{-1}$ korrelierte mit der hohen ESI-Signalintensität und bestätigte eine hohe lonenkonzentration in Lösung.

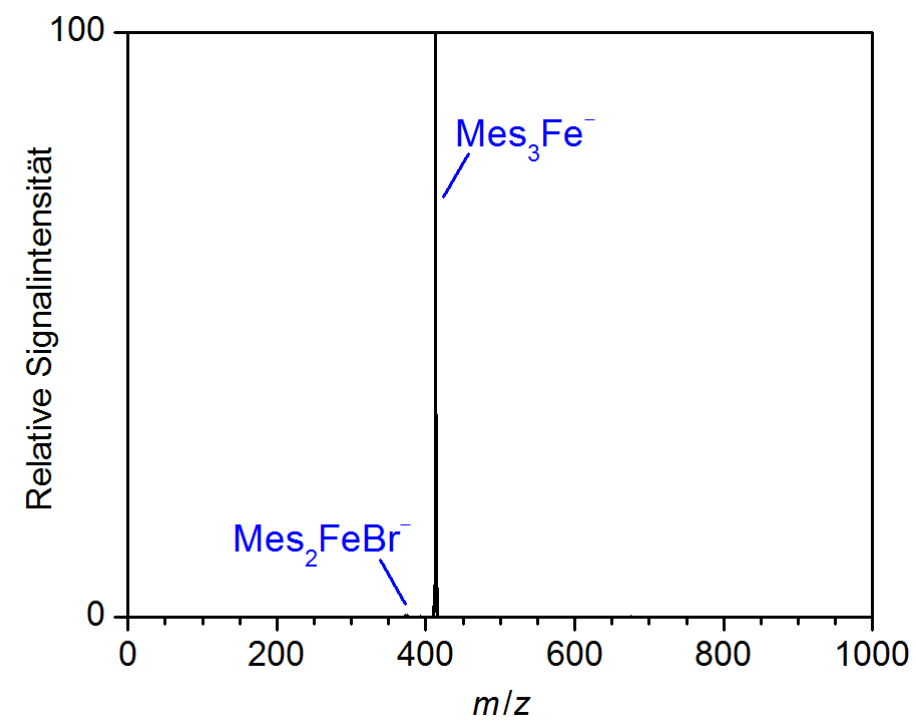

Abbildung 15. ESI-Massenspektrum (negativer lonenmodus) der Produkte aus der Reaktion von $\mathrm{Fe}(\mathrm{acac})_{3}$ (20 mM) und MesMgBr (4.0 Äq.) in THF.

Mesitylferrate im Allgemeinen waren in der Vergangenheit bereits Gegenstand chemischer Forschungen. ${ }^{[76]}$ Speziell $\mathrm{Mes}_{3} \mathrm{Fe}(\mathrm{II})^{-}$konnte mit Röntgenstrukturanalyse und MößbauerSpektroskopie bereits detailliert untersucht werden.[39a,b,76e] Eine Mößbauer-Analyse der Produkte, die über Transmetallierung von ${ }^{57} \mathrm{FeCl}_{2}$ mit $\mathrm{MesMgBr}$ (4.0 Äq.) gebildet wurden, 
zeigte, dass neben $\mathrm{Mes}_{3} \mathrm{Fe}(\mathrm{II})^{-}$keine relevanten Eisen-Spezies in Lösung vorhanden waren $(\rightarrow$ Abbildung 16).

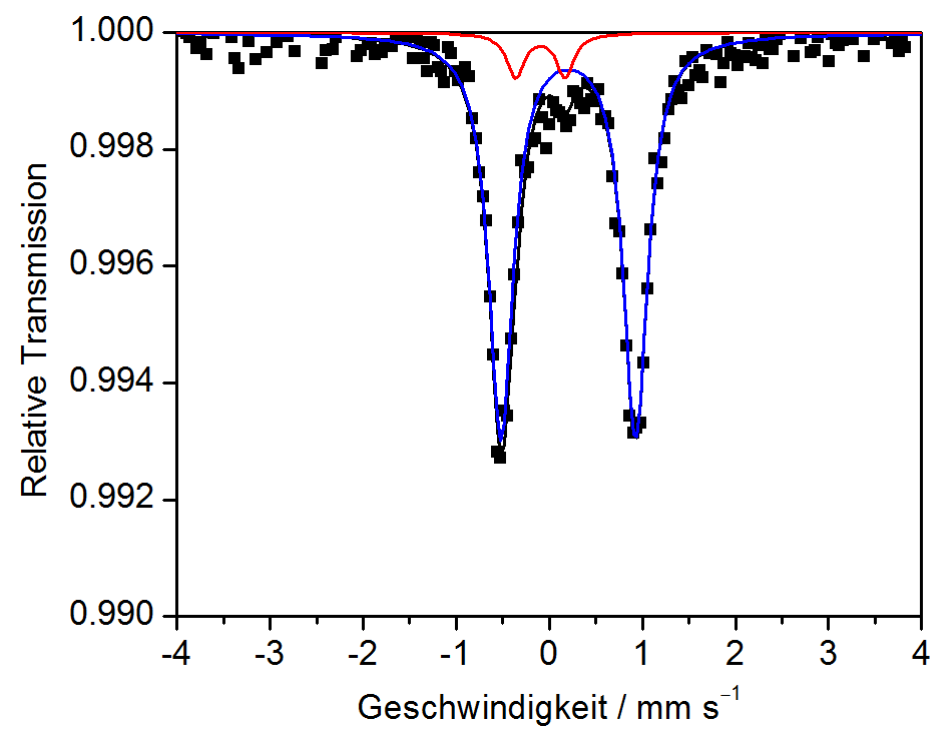

Abbildung 16. Mößbauer-Spektrum einer gefrorenen Lösung der Produkte, entstanden aus der Reaktion von ${ }^{57} \mathrm{FeCl}_{2}$ mit MesMgBr (4.0 Äq.) in THF ( $\left.T=80 \mathrm{~K}\right) ; \delta$ (blau) $=0.20 \mathrm{~mm} \mathrm{~s}^{-1}, \Delta E_{\mathrm{Q}}$ (blau) $=1.45 \mathrm{~mm} \mathrm{~s}^{-1}$, rel. Intensität $($ blau $)=93 \% ; \delta($ rot $)=-0.10 \mathrm{~mm} \mathrm{~s}^{-1}, \Delta E_{Q}($ rot $)=0.53 \mathrm{~mm} \mathrm{~s}^{-1}$, rel. Intensität $($ rot $)=7 \%$.

Neben den Mesitylferraten wurden Arylferrate untersucht, die durch Transmetallierung von $\mathrm{Fe}(\mathrm{acac})_{3}$ mit $\left(\mathrm{Ar}_{\mathrm{F}}\right) \mathrm{MgBr}\left(\mathrm{Ar}_{\mathrm{F}}=3,5-\left(\mathrm{CF}_{3}\right)_{2}-\mathrm{C}_{6} \mathrm{H}_{3}\right)$ geformt werden konnten. So zeigten sich im resultierenden ESI-Massenspektrum im negativen lonenmodus das Arylferrat $\left(\mathrm{Ar}_{\mathrm{F}}\right)_{4} \mathrm{Fe}(\mathrm{III})^{-}$in hoher und die Eisen(II)-Spezies $\left(\mathrm{Ar}_{\mathrm{F}}\right)_{3} \mathrm{Fe}^{-}$und $\left(\mathrm{Ar}_{\mathrm{F}}\right)_{2} \mathrm{FeBr}^{-}$in niedriger relativer Signalintensität ( $\rightarrow$ Abbildung 17). Zudem belegten Messungen der elektrischen Leitfähigkeit, dass diese Arylferrate in Kombination mit ihren Mg-haltigen Gegenionen mit $\kappa=450 \pm 6 \mu \mathrm{sm}^{-1}$ die höchste Leitfähigkeit aller untersuchten Probenlösungen aufwiesen. Das entsprechende Mößbauer-Spektrum einer gefrorenen Lösung von ${ }^{57} \mathrm{FeCl}_{2}$ und $\left(\mathrm{Ar}_{\mathrm{F}}\right) \mathrm{MgBr}$ (4.0 Äq.) in THF zeigte die Anwesenheit von zwei oder mehr eisenhaltigen Spezies, entzieht sich wegen komplexer magnetischer Einflüsse aber einer detaillierteren Untersuchung ( $\rightarrow$ Abbildung 18). Auch eine Verringerung der Temperatur im Mößbauer-Experiment von $T=80 \mathrm{~K}$ auf $T=3 \mathrm{~K}$ konnte die magnetischen Einflüsse auf das Mößbauer-Spektrum nicht vermindern. 


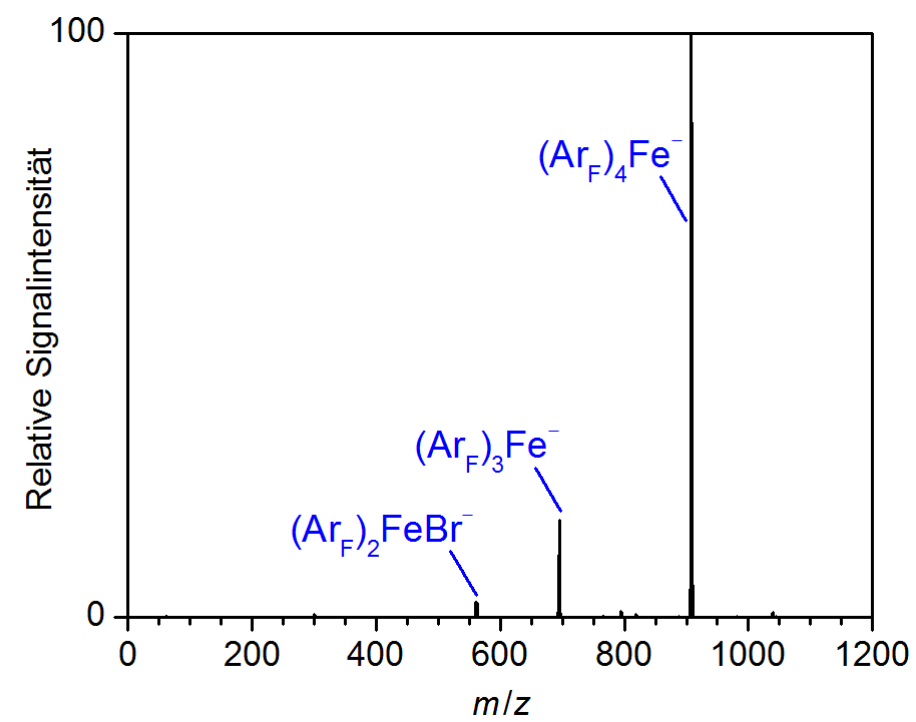

Abbildung 17. ESI-Massenspektrum (negativer lonenmodus) der Produkte aus der Reaktion von $\mathrm{Fe}(\mathrm{acac})_{3}$ (20 mM) mit (ArF) $\mathrm{MgBr}\left(4.0\right.$ Äq.) in $\mathrm{THF} ; \mathrm{Ar}_{\mathrm{F}}=3,5-\left(\mathrm{CF}_{3}\right)_{2}-\mathrm{C}_{6} \mathrm{H}_{3}$.

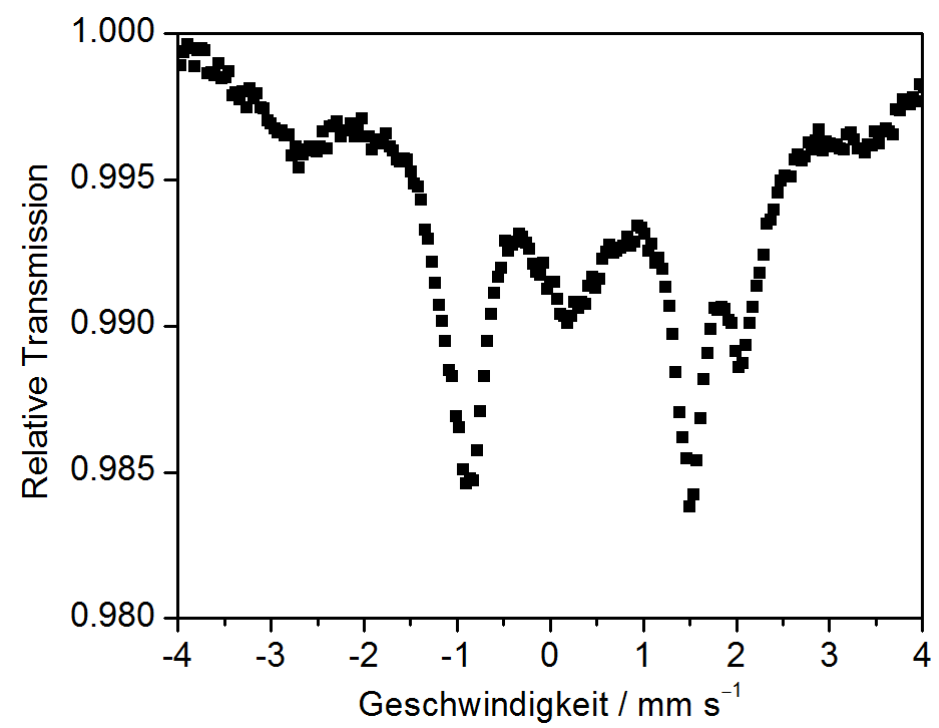

Abbildung 18. Mößbauer-Spektrum einer gefrorenen Lösung der Produkte aus der Reaktion von ${ }^{57} \mathrm{FeCl}_{2} \mathrm{mit}$ $\left(\mathrm{Ar}_{\mathrm{F}}\right) \mathrm{MgBr}(4.0 \mathrm{Äq}$.) in THF $(T=80 \mathrm{~K})$; eine Analyse des Spektrums war aufgrund der komplexen magnetischen Einflüsse nicht möglich; $\mathrm{ArF}=3,5-\left(\mathrm{CF}_{3}\right)_{2}-\mathrm{C}_{6} \mathrm{H}_{3}$. 


\subsubsection{Transmetallierung von $\mathrm{Fe}(\mathrm{acac}) 3$ mit $\mathrm{PhMgCl}$ und $\mathrm{RMgBr}$}

Ausgehend von einer Lösung von $\mathrm{Fe}(\mathrm{acac})_{3}$ in THF ließ sich durch simultane Transmetallierung mit PhMgCl und $\mathrm{RMgX}$ eine Vielzahl an gemischten Arylferraten erzeugen. Dadurch war es möglich, Aussagen über sterische und elektronische Einflüsse auf die Bildung und die Stabilität von Arylferraten zu treffen.

$\mathrm{Zu}$ Beginn erfolgte die Transmetallierung mit $\mathrm{PhMgCl}$ (2.0 Äq.) und einem aromatischen Grignard-Reagenz $\mathrm{RMgBr}$ mit einer stark elektronenschiebenden $\mathrm{NMe}_{2}$-Gruppe in para-Position (2.0 Äq., $\mathrm{R}=p-\mathrm{Me}_{2} \mathrm{~N}-\mathrm{C}_{6} \mathrm{H}_{4}$ ). Neben den bereits bekannten mononuklearen Phenylferraten $\mathrm{Ph}_{3} \mathrm{Fe}(\mathrm{II})^{-}$und $\mathrm{Ph}_{4} \mathrm{Fe}(\mathrm{III})^{-}$konnten die gemischten Organoferrate $\mathrm{Ph}_{2} \mathrm{Fe}(\mathrm{II}) \mathrm{R}^{-}$ und $\mathrm{Ph}_{3} \mathrm{Fe}(\mathrm{III}) \mathrm{R}^{-}$massenspektrometrisch in geringer und schnell abnehmender Signalintensität beobachtet werden. Das Einführen einer weniger stark elektronenschiebenden Methoxy-Gruppe in para-Position des arylischen Grignard-Reagenzes $\mathrm{RMgBr}\left(\mathrm{R}=p-\mathrm{MeO}-\mathrm{C}_{6} \mathrm{H}_{4}\right)$ ermöglichte das Beobachten analoger Spezies, wobei zusätzlich $\mathrm{PhFe}(\mathrm{II}) \mathrm{R}_{2}^{-}$zugänglich war ( $\rightarrow$ Abbildung 19 ).

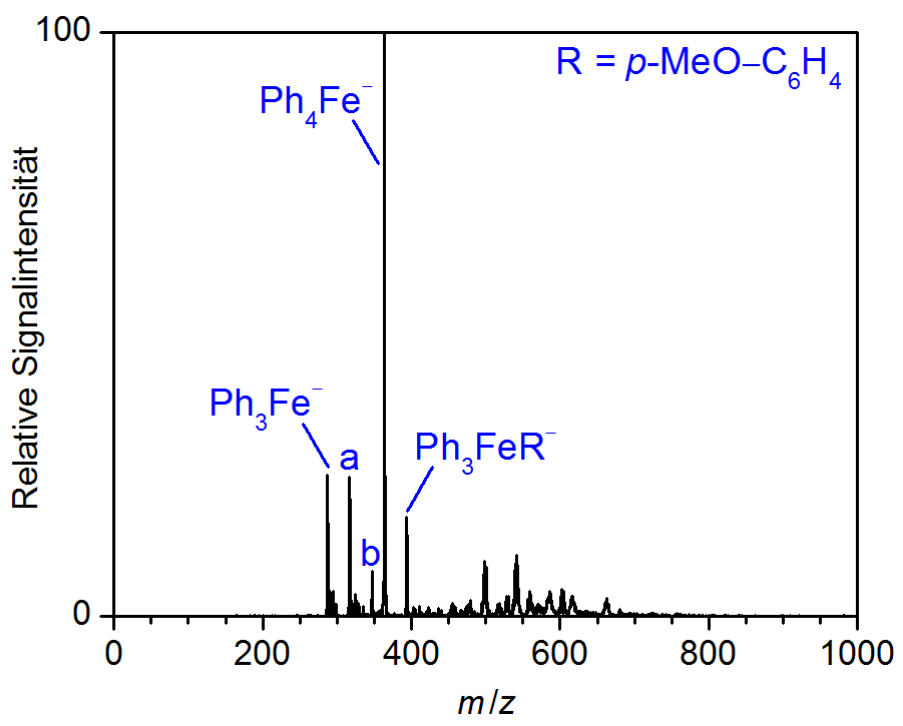

Abbildung 19. ESI-Massenspektrum (negativer lonenmodus) der Produkte aus der Reaktion von $\mathrm{Fe}(\mathrm{acac})_{3}$ (20 mM) mit PhMgCl (2.0 Äq.) und $\mathrm{RMgBr}\left(2.0\right.$ Äq.) in THF; $\mathrm{a}=\mathrm{Ph}_{2} \mathrm{FeR}^{-}, \mathrm{b}=\mathrm{PhFeR}_{2}^{-} ; \mathrm{R}=p-\mathrm{MeO}^{-} \mathrm{C}_{6} \mathrm{H}_{4}$.

Für $\mathrm{R}=p$-Tol konnten die vollständigen Serien $\mathrm{Ph}_{n} \mathrm{Fe}(\mathrm{II}) \mathrm{R}_{3-n^{-}}(n=0-3)$ und $\mathrm{Ph}_{n} \mathrm{Fe}(\mathrm{III}) \mathrm{R}_{4-n^{-}}$ $(n=0-4)$ in hoher und zeitlich stabiler Signalintensität detektiert werden ( $\rightarrow$ Abbildung 20$)$. Der Übergang zu para-Substituenten mit elektronenziehendem Effekt bewirkte, dass ausschließlich die Eisen(III)-Serie $\mathrm{Ph}_{n} \mathrm{FeR}_{4-n^{-}}\left(n=0-4, \mathrm{R}=p-\mathrm{F}_{-}-\mathrm{C}_{6} \mathrm{H}_{4}\right)$ im resultierenden ESIMassenspektrum im negativen lonenmodus auftrat ( $\rightarrow$ Abbildung 21). Arylferrate mit Eisen in der Oxidationsstufe +II konnten hier nicht mehr beobachtet werden. 


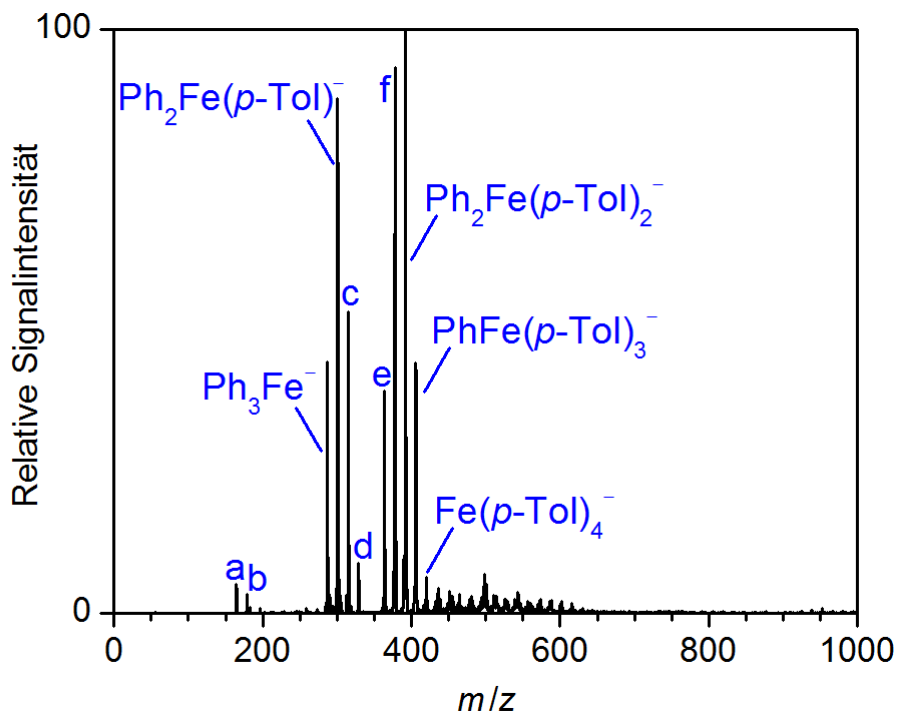

Abbildung 20. ESI-Massenspektrum (negativer lonenmodus) der Produkte aus der Reaktion von $\mathrm{Fe}(\mathrm{acac})_{3}$ $(20 \mathrm{~mm})$ mit $\mathrm{PhMgCl}$ (2.0 Äq.) und ( $p$ - Tol) $\mathrm{MgBr}$ (2.0 Äq.) in THF; $a=\left[\mathrm{Ph}, \mathrm{Fe}, \mathrm{O}_{2}\right]^{-}, \quad b=\left[\mathrm{Fe},(p-\mathrm{Tol}), \mathrm{O}_{2}\right]^{-}$, $c=\operatorname{PhFe}(p-\mathrm{Tol}) 2^{-}, \mathrm{d}=\mathrm{Fe}(p-\mathrm{Tol}) 3^{-}, \mathrm{e}=\mathrm{Ph}_{4} \mathrm{Fe}^{-}, \mathrm{f}=\mathrm{Ph}_{3} \mathrm{Fe}(p-\mathrm{Tol})^{-}$.

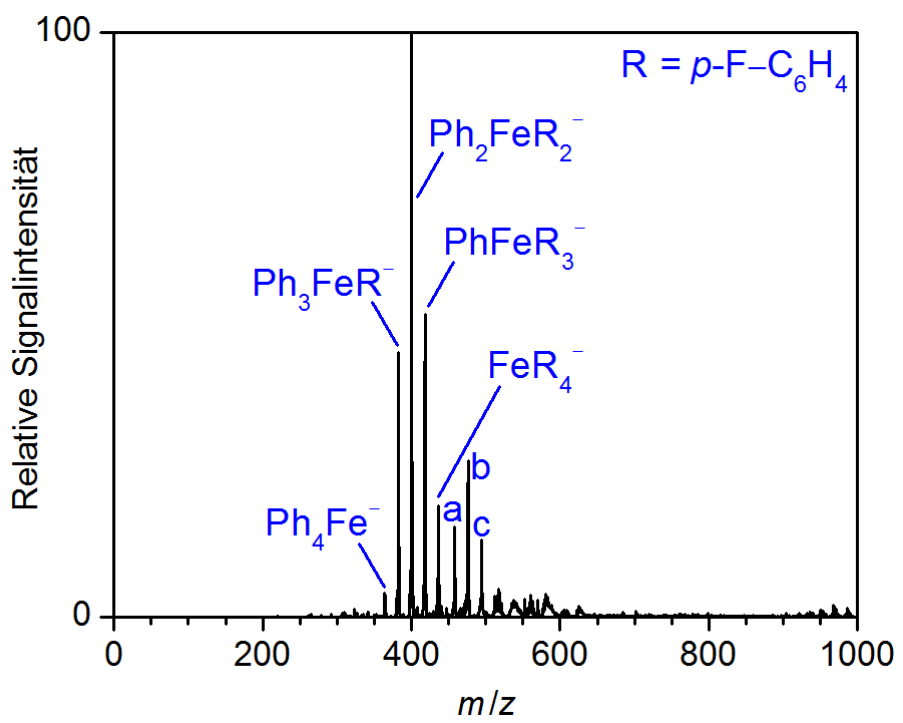

Abbildung 21. ESI-Massenspektrum (negativer lonenmodus) der Produkte aus der Reaktion von $\mathrm{Fe}(\mathrm{acac})_{3}$ (20 mM) mit PhMgCl (2.0 Äq.) und RMgBr (2.0 Äq.) in THF; $\mathrm{a}=\mathrm{Ph}_{3} \mathrm{Fe}\left(\mathrm{C}_{6} \mathrm{H}_{4} \mathrm{C}_{6} \mathrm{H}_{4} \mathrm{~F}\right)^{-}, \mathrm{b}=\mathrm{Ph}_{2} \mathrm{FeR}\left(\mathrm{C}_{6} \mathrm{H}_{4} \mathrm{C}_{6} \mathrm{H}_{4} \mathrm{~F}\right)^{-}$, $\mathrm{C}=\mathrm{PhFeR}_{2}\left(\mathrm{C}_{6} \mathrm{H}_{4} \mathrm{C}_{6} \mathrm{H}_{4} \mathrm{~F}\right)^{-} ; \mathrm{R}=p-\mathrm{F}-\mathrm{C}_{6} \mathrm{H}_{4}$. Die Spezies a-c entstanden durch eine Nebenreaktion. 
Wurde der elektronenziehende Effekt des $p$-Substituenten weiter erhöht, dominierten vermehrt die phenylarmen Arylferrate mit Eisen(III)-Zentrum. So bewirkte die gleichzeitige Transmetallierung von $\mathrm{Fe}(\mathrm{acac})_{3}$ mit $\mathrm{PhMgCl}$ (2.0 Äq.) und $\mathrm{RMgBr}\left(2.0 \mathrm{Äq}\right.$., $\mathrm{R}=p-\mathrm{F}_{3} \mathrm{C}-\mathrm{C}_{6} \mathrm{H}_{4}$ oder $\left.\mathrm{R}=p-\mathrm{CN}-\mathrm{C}_{6} \mathrm{H}_{4}\right)$ ein hohes Aufkommen an $\mathrm{PhFe}(\mathrm{III}) \mathrm{R}_{3}{ }^{-}\left(\mathrm{R}=p-\mathrm{F}_{3} \mathrm{C}-\mathrm{C}_{6} \mathrm{H}_{4}\right)$ und $\mathrm{Fe}(\mathrm{III}) \mathrm{R}_{4}{ }^{-}$ $\left(\mathrm{R}=p-\mathrm{F}_{3} \mathrm{C}-\mathrm{C}_{6} \mathrm{H}_{4}\right.$ oder $\mathrm{R}=p-\mathrm{CN}-\mathrm{C}_{6} \mathrm{H}_{4} ; \rightarrow$ Abbildung 22). Erwähnenswert war insbesondere das Auftreten der organischen Eisen(IV)-Spezies $\mathrm{Ph}_{n} \mathrm{FeR}_{5-n^{-}}(n=0,1)$ im Falle einer $\mathrm{CF}_{3}$-Gruppe als para-Substituent.

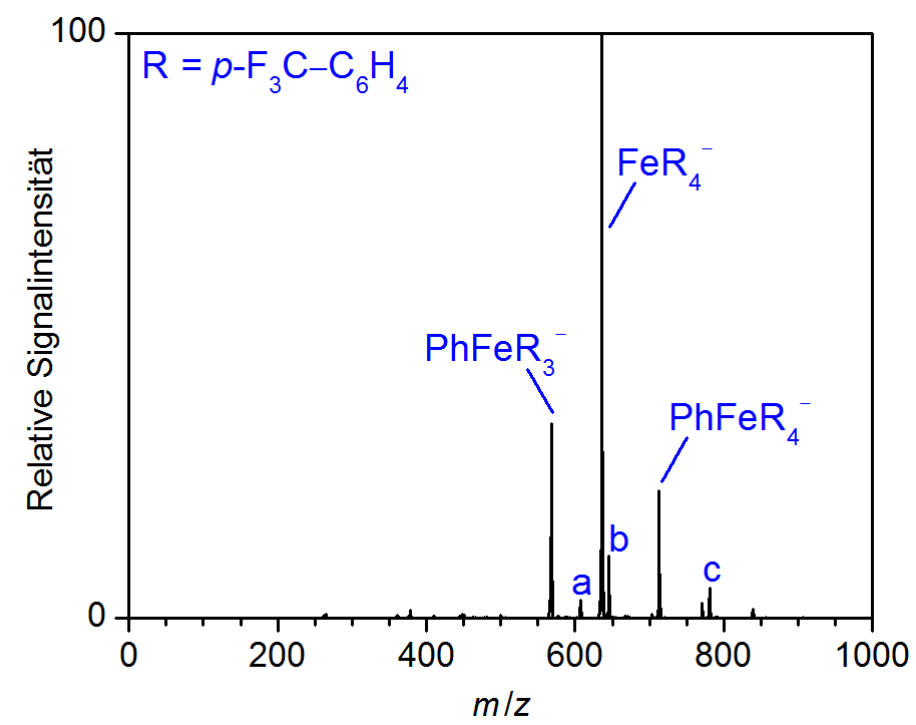

Abbildung 22. ESI-Massenspektrum (negativer lonenmodus) der Produkte aus der Reaktion von $\mathrm{Fe}(\mathrm{acac})_{3}$ (20 mM) mit PhMgCl (2.0 Äq.) und RMgBr (2.0 Äq.) in THF; $a=\mathrm{AlR}_{4}{ }^{-}, \mathrm{b}=\mathrm{Ph}_{2} \mathrm{FeR}_{3}{ }^{-}, \mathrm{c}=\mathrm{FeR}_{5}{ }^{-} ; \mathrm{R}=p-\mathrm{F}_{3} \mathrm{C}-\mathrm{C}_{6} \mathrm{H}_{4}$.

Zuletzt wurden simultane Transmetallierungsexperimente genutzt, um den sterischen Einfluss auf die Bildung und die Stabilität von Organoferraten besser zu verstehen. So bewirkte die Reaktion von $\mathrm{Fe}(\mathrm{acac})_{3}$ mit $\mathrm{PhMgCl}$ und $\mathrm{RMgBr}(\mathrm{R}=0$-Tol), dass Arylferrate vom Typ $\mathrm{Ph}_{n} \mathrm{FeR}_{3-n^{-}}(n=0-3)$ mit Eisen(II)-Zentrum in besonders hoher und zeitlich stabiler Signalintensität vorlagen ( $\rightarrow$ Abbildung 23). Das Phänomen der Dominanz dieser Eisen(II)Serie konnte auch in weiteren Experimenten $\left(\mathrm{R}=\mathrm{o}-\mathrm{F}_{3} \mathrm{C}-\mathrm{C}_{6} \mathrm{H}_{4}\right.$, Mes) beobachtet werden. $\mathrm{Zu}$ betonen ist dabei, dass für $\mathrm{R}=$ Mes die mesityl- gegenüber den phenylreichen Spezies klar dominierten. An dieser Tatsache änderte auch die Erhöhung der Äquivalente an $\mathrm{PhMgCl}$ im Vergleich zu MesMgBr (10:1) nur wenig. 


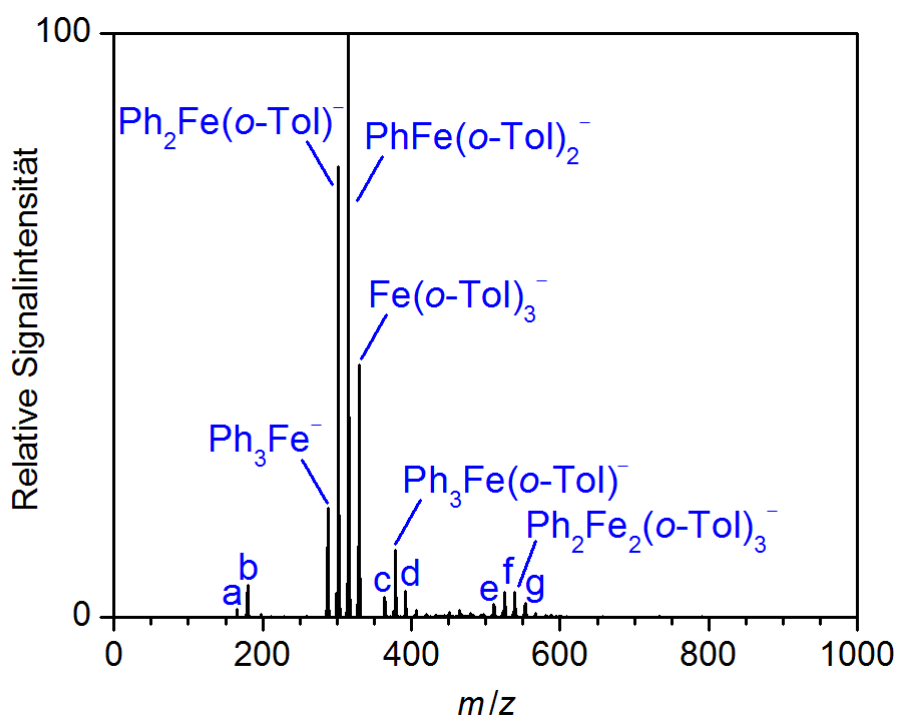

Abbildung 23. ESI-Massenspektrum (negativer lonenmodus) der Produkte aus der Reaktion von $\mathrm{Fe}(\mathrm{acac})_{3}$ (20 mM) mit PhMgCl (2.0 Äq.) und (o-Tol)MgBr (2.0 Äq.) in THF; a = $\left[\mathrm{Ph}, \mathrm{Fe}, \mathrm{O}_{2}\right]^{-}, \mathrm{b}=\left[\mathrm{Fe},(\mathrm{O}-\mathrm{Tol}), \mathrm{O}_{2}\right]^{-}, \mathrm{c}=\mathrm{Ph}_{4} \mathrm{Fe}^{-}$, $\mathrm{d}=\mathrm{Ph}_{2} \mathrm{Fe}(o-\mathrm{Tol}) 2^{-}, \mathrm{e}=\mathrm{Ph}_{4} \mathrm{Fe}_{2}(o-\mathrm{Tol})^{-}, \mathrm{f}=\mathrm{Ph}_{3} \mathrm{Fe}_{2}\left(o-\mathrm{Tol}_{2}{ }^{-}, \mathrm{g}=\mathrm{PhFe}_{2}(o-\mathrm{Tol}) 4^{-}\right.$. 


\subsubsection{Transmetallierung von $\mathrm{FeX}_{2} / \mathrm{FeX}_{3}$ mit $\mathrm{MeMgX}$}

Über die Reaktion von $\mathrm{Fe}(\mathrm{acac})_{3}$ mit $\mathrm{MeMgCl}\left(4.0 \mathrm{Äq}\right.$.) war das achtkernige Aggregat $\mathrm{Me}_{12} \mathrm{Fe}_{8}{ }^{-}$ mit Eisen in der durchschnittlichen Oxidationsstufe von 1.4 zugänglich ( $\rightarrow$ Abbildung 24 und 25). Zusätzlich ermöglichte die ESI-massenspektrometrische Untersuchung im negativen Ionenmodus die Detektion der mononuklearen Methylferrate $\mathrm{Me}_{3} \mathrm{Fe}(\mathrm{II})^{-}$und $\mathrm{Me}_{4} \mathrm{Fe}(\mathrm{III})^{-}$in niedriger und schnell abfallender Signalintensität. Im hohen Massenbereich waren zudem die Spezies $\mathrm{Me}_{13} \mathrm{Fe}_{8}{ }^{-}$und $\left[\mathrm{Me}_{12}, \mathrm{Fe}_{8}, \mathrm{O}_{2}\right]^{-}$zu erkennen. Die Bildung des polynuklearen Anions $\mathrm{Me}_{12} \mathrm{Fe}_{8}{ }^{-}$erschien auf den ersten Blick ungewöhnlich. Das Vorliegen eines solch hohen Aggregates konnte aber durch das analoge Transmetallierungsexperiment mit $\mathrm{CD}_{3} \mathrm{Mgl}$ und der daraus resultierenden Entstehung von $\left(\mathrm{CD}_{3}\right)_{12} \mathrm{Fe}_{8}{ }^{-}$bestätigt werden. Zudem konnten Neidig und Mitarbeiter 2016 den achtkernigen Methyl-Eisencluster mittels RöntgenKristallstrukturanalyse als heterokubane Struktur eindeutig identifizieren und charakterisieren ${ }^{[399]}$ Interessant war, dass die Methylferrate zusammen mit ihren Mg-haltigen Gegenionen mit $\kappa=65 \pm 17 \mu \mathrm{S} \mathrm{cm}{ }^{-1}$ den niedrigsten Leitfähigkeitswert aller untersuchten Organoferrat-Systeme aufwiesen.

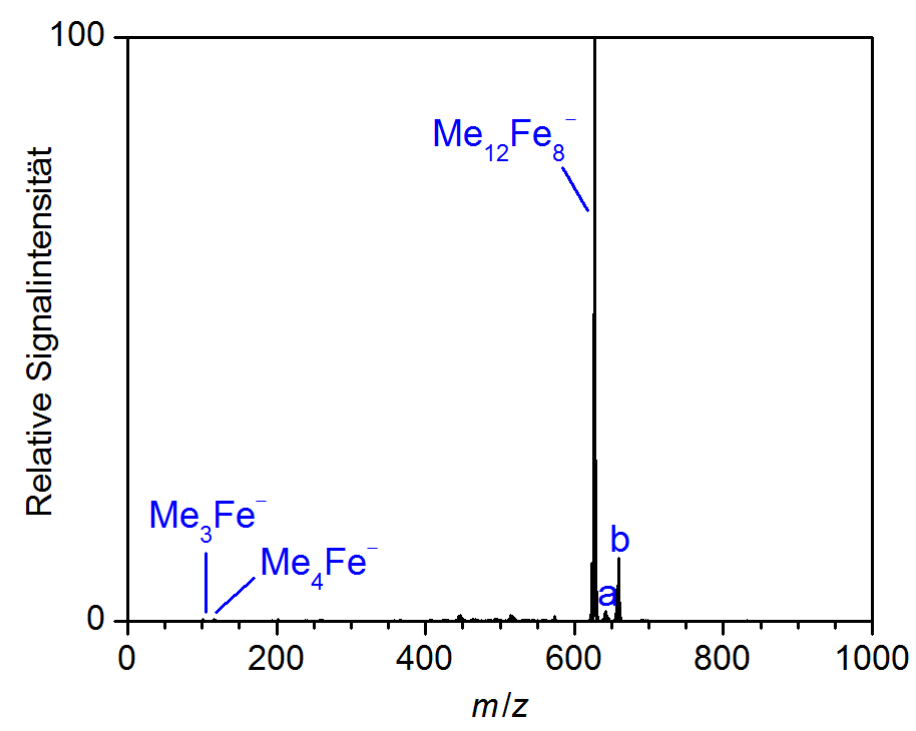

Abbildung 24. ESI-Massenspektrum (negativer lonenmodus) der Produkte aus der Reaktion von $\mathrm{Fe}(\mathrm{acac})_{3}$ (20 mM) mit $\mathrm{MeMgCl}$ (4.0 Äq.) in THF; $a=\mathrm{Me}_{13} \mathrm{Fe}_{8}{ }^{-}, \mathrm{b}=\left[\mathrm{Me}_{12}, \mathrm{Fe}_{8}, \mathrm{O}_{2}\right]^{-}$. 


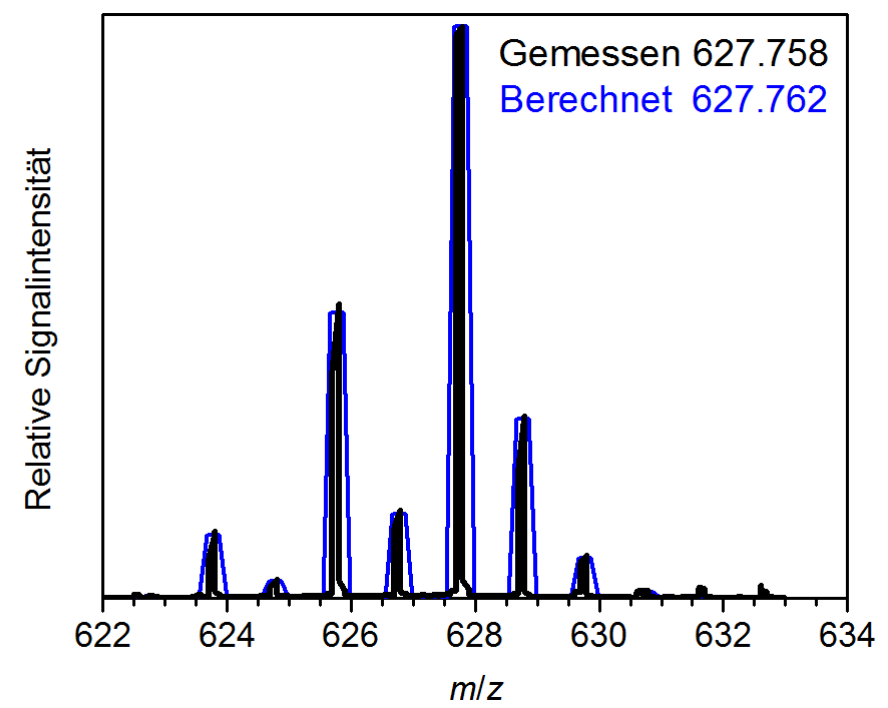

Abbildung 25. Vergleich des gemessenen (schwarz) und des simulierten (blau) Isotopenmusters von $\mathrm{Me}_{12} \mathrm{Fe}_{8}{ }^{-}$.

Wie schon bei der Transmetallierung mit $\mathrm{PhMgCl}$ wurden verschiedene Einflussfaktoren auf die analoge Reaktion mit $\mathrm{MeMgCl}$ überprüft. So konnte auch für die Methylferrate als einfachsten Vertreter der Alkylferrate gezeigt werden, dass weder die Wahl des Eisenvorläufers noch der Austausch von $\mathrm{MeMgCl}$ durch $\mathrm{MeMgBr}$ einen entscheidenden Effekt auf die Bildung der Ferrat-Komplexe hatte. Erneut konnte belegt werden, dass die elektrische Leitfähigkeit nicht von der Oxidationsstufe des Eisenvorläufers, sondern nur von dessen Gegenion $X^{-}$abhängig war ( $\rightarrow$ Tabelle 4$)$.

Tabelle 4. Spezifische elektrische Leitfähigkeit $\kappa / \mu \mathrm{S} \mathrm{cm}^{-1}$ von Methylferraten und ihren Mg-haltigen Gegenionen gebildet über Transmetallierung verschiedener Eisenvorläufer (50 mM) mit $\mathrm{MeMgCl}$ (4.0 Äq.) in THF bei $T=273 \mathrm{~K}$.

\begin{tabular}{lr}
\hline \multicolumn{1}{c}{ Probenlösung in THF } & $\boldsymbol{\kappa} /\left(\boldsymbol{\mu} \mathbf{~ c m}^{-1}\right)$ \\
\hline $\mathrm{Fe}(\mathrm{acac})_{3}+4 \mathrm{MeMgCl}$ & $65 \pm 17$ \\
$\mathrm{FeCl}_{3}+4 \mathrm{MeMgCl}$ & $206 \pm 17$ \\
$\mathrm{FeCl}_{2}+4 \mathrm{MeMgCl}$ & $222 \pm 30$ \\
$\mathrm{Fe}(\mathrm{OAc})_{2}+4 \mathrm{MeMgCl}$ & $141 \pm 46$ \\
\hline
\end{tabular}


Das Mößbauer-Experiment einer gefrorenen Reaktionslösung von ${ }^{57} \mathrm{FeCl}_{2}$ und $\mathrm{MeMgCl}$ (4.0 Äq.) zeigte große Übereinstimmungen mit dem Spektrum, das 2016 von Neidig und Mitarbeitern publiziert wurde ( $\rightarrow$ Abbildung 26 ). ${ }^{[39 g]}$ Beide Experimente resultierten in einem Hauptpeak, der als breites, aber symmetrisches Dublett erkennbar war. Hier schienen magnetische Effekte nicht die Ursache der Signalverbreiterung zu sein. Aus den Kristallstrukturdaten von Neidig und Mitarbeitern wurde deutlich, dass in der heterokubanen Struktur von $\mathrm{Me}_{12} \mathrm{Fe}_{8}{ }^{-}$im Wesentlichen zwei Arten von Eisenkernen vorlagen. Diese liegen in einer ähnlichen, aber nicht identischen elektronischen Umgebung vor, weshalb auch die Mößbauer-Signale der entsprechenden Eisen-Zentren ähnliche, aber folglich keine identischen Mößbauer-Parameter aufweisen sollten. Somit lässt sich durch Überlagerung zweier Mößbauer-Dubletts die symmetrische Verbreiterung des resultierenden MößbauerSignals erklären.

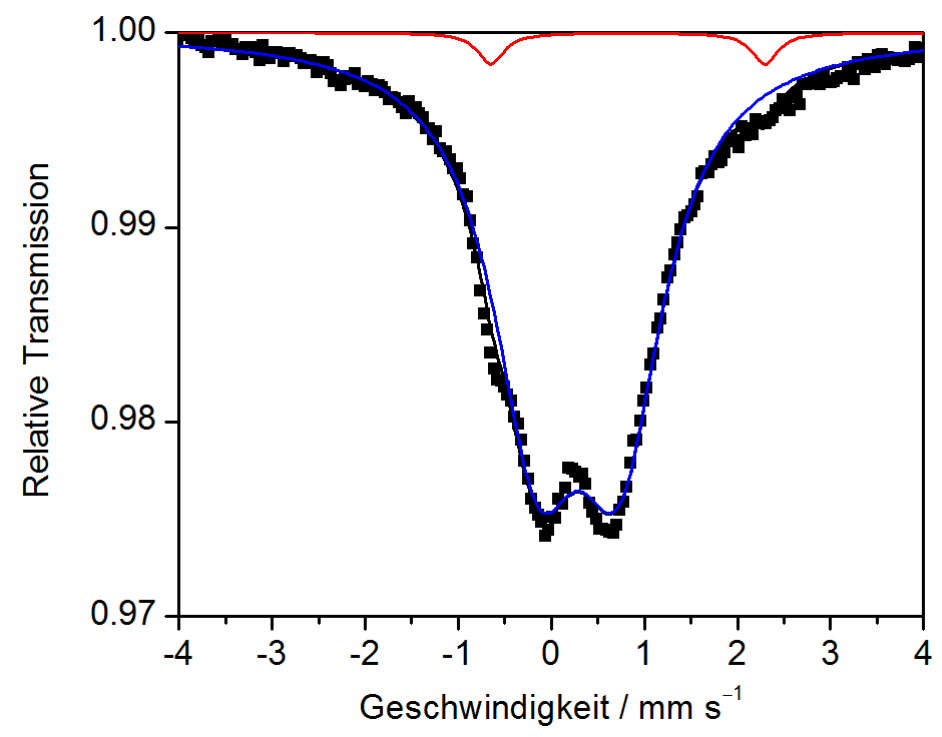

Abbildung 26. Mößbauer-Spektrum einer gefrorenen Lösung der Produkte aus der Reaktion von ${ }^{57} \mathrm{FeCl}_{2}$ mit $\mathrm{MeMgCl}$ (4.0 Äq.) in THF ( $T=80 \mathrm{~K}) ; \delta$ (blau) $=0.29 \mathrm{~mm} \mathrm{~s}^{-1}, \Delta E_{\mathrm{Q}}$ (blau) $=0.89 \mathrm{~mm} \mathrm{~s}^{-1}$, rel. Intensität (blau) $=97 \%$; $\delta($ rot $)=0.82 \mathrm{~mm} \mathrm{~s}^{-1}, \Delta E_{\mathrm{Q}}($ rot $)=2.94 \mathrm{~mm} \mathrm{~s}^{-1}$, rel. Intensität (rot) $=3 \%$. 


\subsubsection{Transmetallierung von $\mathrm{Fe}(\mathrm{acac})_{3}$ mit AlkMgCl}

Neben den Methylferraten wurden auch die Eisen-Spezies genauer untersucht, die über Transmetallierung von $\mathrm{Fe}(\mathrm{acac})_{3}$ mit den höheren Homologen der aliphatischen GrignardReagenzien $\mathrm{RMgCl}(\mathrm{R}=\mathrm{Et}, \mathrm{Bu}, \mathrm{Hex}, \mathrm{Oct}, \mathrm{Dec})$ erzeugt werden konnten. Ethylferrate und Butylferrate waren nicht stabil genug, um mittels ESI-Massenspektrometrie detektiert werden zu können. Die entsprechende Reaktion mit $\mathrm{HexMgCl}$ ermöglichte die kurzzeitige Detektion von $\mathrm{Hex}_{4} \mathrm{Fe}(\mathrm{III})^{-}$. Durch Einsatz von OctMgCl oder DecMgCl konnte eine Dominanz der analogen $\mathrm{Fe}(\mathrm{III})$-Spezies $\mathrm{R}_{4} \mathrm{Fe}^{-}(\mathrm{R}=\mathrm{Oct}$, Dec) im Negativ-lonen-ESI-Massenspektrum beobachtet werden ( $\rightarrow$ Abbildung 27). Daneben war in geringen Spuren $\mathrm{R}_{3} \mathrm{Fe}(\mathrm{II})^{-}(\mathrm{R}=$ Oct, Dec) zu erkennen. Signifikant war die zeitlich geringe Stabilität aller Alkylferrate, die zu einem raschen Absinken der Signalintensität in den Massenspektren führte.

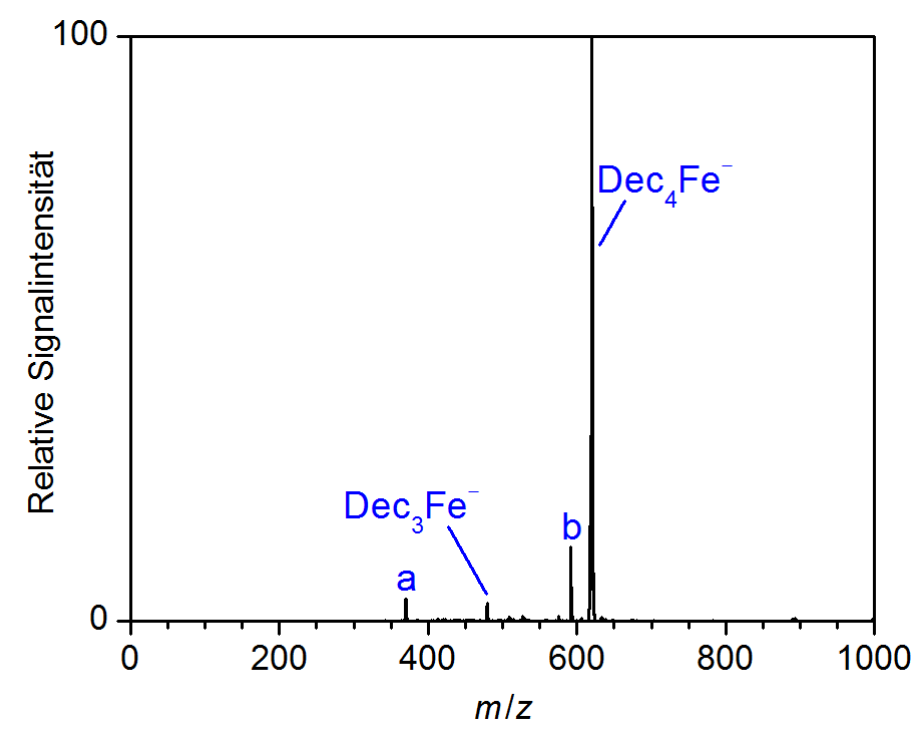

Abbildung 27. ESI-Massenspektrum (negativer lonenmodus) der Produkte aus der Reaktion von $\mathrm{Fe}(\mathrm{acac})_{3}$ (20 mm) mit DecMgCl (4.0 Äq.) in THF; $a=\left[\mathrm{Dec}, \mathrm{Fe}, \mathrm{O}_{2}\right]^{-}, \mathrm{b}=\mathrm{Dec}_{4} \mathrm{Al}^{-}$.

Repräsentativ für alle Alkylferrate mit $\beta$-H-Atomen wurden die Eisen-Spezies, die über Transmetallierung von ${ }^{57} \mathrm{FeCl}_{2}$ mit DecMgCl gebildet werden konnten, zusätzlich mittels Mößbauer-Spektroskopie untersucht. Wie im ESI-Massenspektrum war auch hier die Dominanz einer Eisen(III)-Spezies offensichtlich ( $\rightarrow$ Abbildung 28). Aufgrund der MößbauerParameter war klar zu erkennen, dass diese in einem high-spin-Zustand vorlag. 


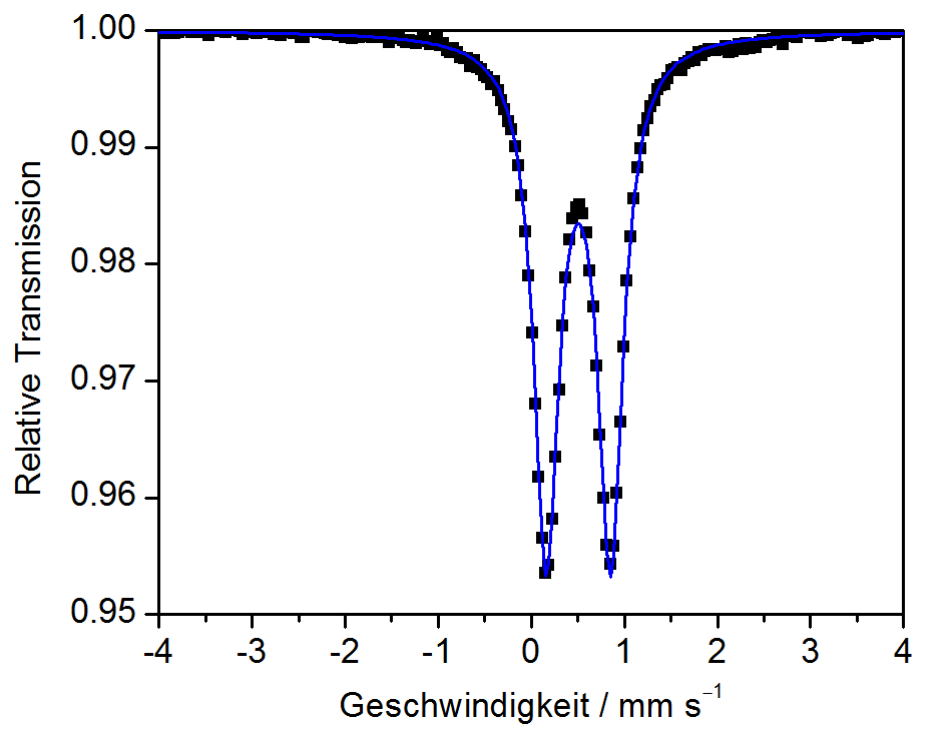

Abbildung 28. Mößbauer-Spektrum einer gefrorenen Lösung der Produkte aus der Reaktion von ${ }^{57} \mathrm{FeCl}_{2} \mathrm{mit}$ DecMgCl (4.0 Äq.) in THF $(T=80 \mathrm{~K}) ; \quad \delta$ (blau) $=0.51 \mathrm{~mm} \mathrm{~s}^{-1}, \Delta E \mathrm{Q}$ (blau) $=0.69 \mathrm{~mm} \mathrm{~s}^{-1}$, rel. Intensität (blau $)=100 \%$. 


\subsubsection{Transmetallierung von $\mathrm{Fe}(\mathrm{acac})_{3}$ mit $\mathrm{BnMgCl}$}

Als weiteres System ohne $\beta$ - $\mathrm{H}$-Atome wurde die Transmetallierung von $\mathrm{Fe}(\mathrm{acac})_{3}$ mit $\mathrm{BnMgCl}$ (4.0 Äq.) detaillierter untersucht. Dabei waren mit $\mathrm{Bn}_{3} \mathrm{Fe}^{-}$und $\mathrm{Bn}_{4} \mathrm{Fe}^{-}$erneut einkernige Spezies mit Eisen(II)- und Eisen(III)-Zentrum die dominierenden Anionen ( $\rightarrow$ Abbildung 29 ). Auch die binuklearen Benzylferrate $\mathrm{Bn}_{5} \mathrm{Fe}_{2}{ }^{-}$und $\mathrm{Bn}_{6} \mathrm{Fe}_{2}{ }^{-}$mit Eisenkernen in den durchschnittlichen Oxidationszahlen +2.0 bzw. +2.5 konnten identifiziert werden. Die Transmetallierung von $\mathrm{Fe}(\mathrm{acac})_{3}$ mit $\mathrm{BnMgCl}$ zeigte sich besonders empfindlich gegenüber der Präsenz von Sauerstoff. So konnten die sauerstoffhaltigen At-Komplexe der Zusammensetzung $\left[\mathrm{Bn}, \mathrm{Fe}, \mathrm{O}_{2}\right]^{-}$und $\left[\mathrm{Bn}_{2}, \mathrm{Fe}, \mathrm{O}_{2}\right]^{-}$in deutlicher Signalintensität beobachtet werden.

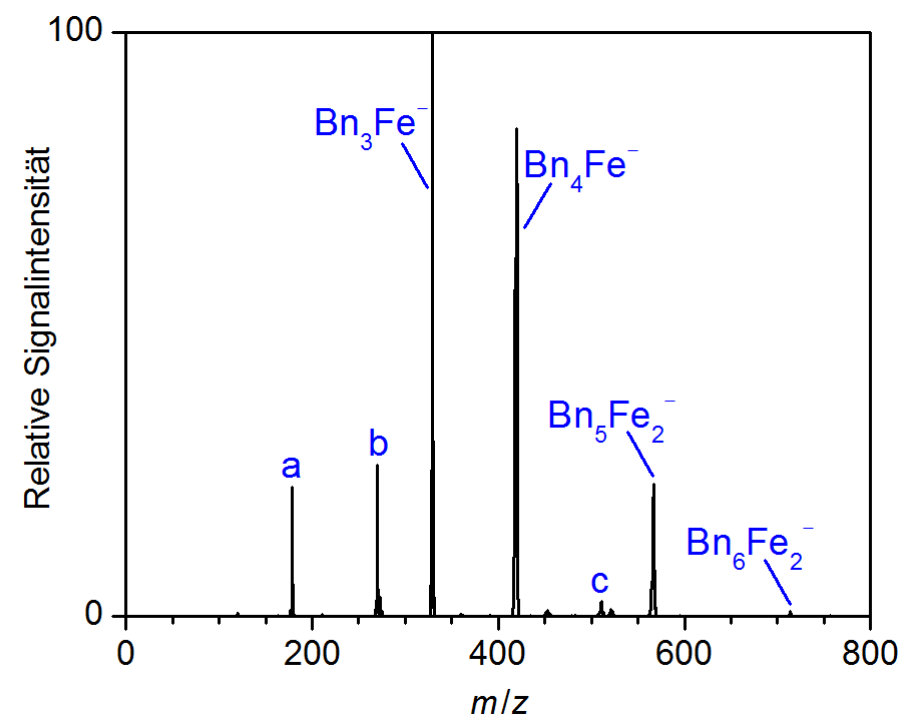

Abbildung 29. ESI-Massenspektrum (negativer lonenmodus) der Produkte aus der Reaktion von $\mathrm{Fe}(\mathrm{acac})_{3}$ (20 mM) mit BnMgCl (4.0 Äq.) in THF; $\mathrm{a}=\left[\mathrm{Bn}, \mathrm{Fe}, \mathrm{O}_{2}\right]^{-}, \mathrm{b}=\left[\mathrm{Bn} 2, \mathrm{Fe}, \mathrm{O}_{2}\right]^{-}, \mathrm{c}=\left[\mathrm{Bn}{ }_{5} \mathrm{Fe}-\mathrm{H}\right]^{-}$.

Die Mößbauer-spektroskopische Untersuchung einer gefrorenen Reaktionslösung von ${ }^{57} \mathrm{FeCl}_{2}$ mit $\mathrm{BnMgCl}$ (4.0 Äq.) in THF bestätigte das vorangegangene Ergebnis ( $\rightarrow$ Abbildung 30 ). Auch hier dominierten Spezies mit Eisen(II)- und Eisen(III)-Zentrum. Für die erstgenannte war ein low-spin-Charakter wahrscheinlich, wohingegen die Eisen(III)-Spezies in einem high-spinZustand vorlag. Auch konnte eine sehr gute quantitative Übereinstimmung beider Analysemethoden beobachtet werden, da sowohl die ESI-Massenspektrometrie, als auch die Mößbauer-Spektroskopie auf ein nahezu identisches Vorhandensein beider Spezies in Lösung hinwiesen. Neben den beiden Hauptspezies war im Mößbauer-Spektrum mit einer relativen Signalintensität von 13\% zusätzlich eine Eisen(II)-Verbindung im high-spin-Zustand detektierbar. 


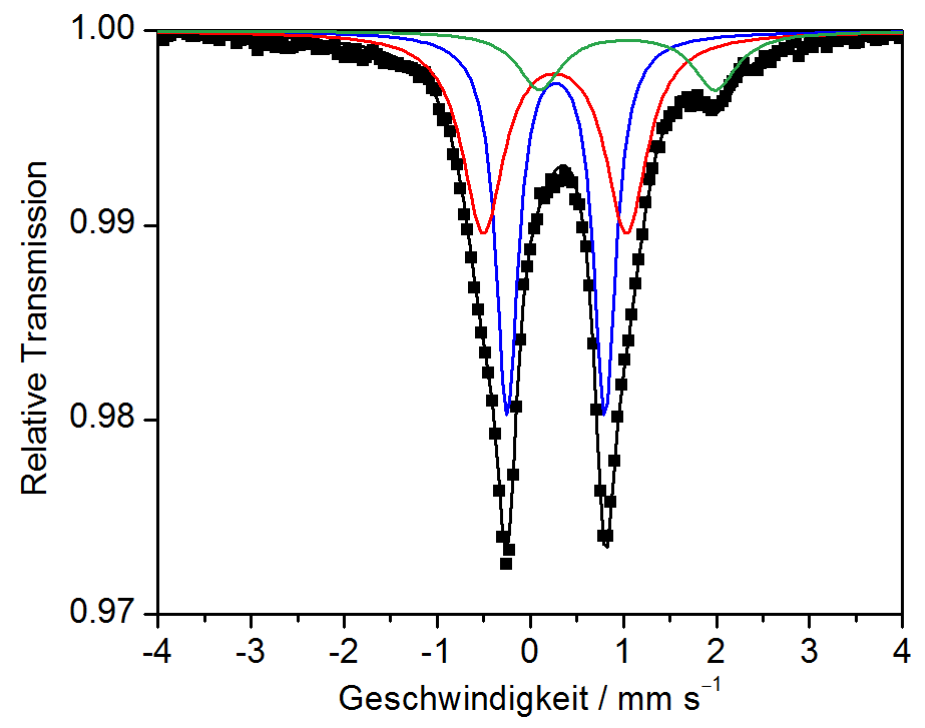

Abbildung 30. Mößbauer-Spektrum einer gefrorenen Lösung der Produkte aus der Reaktion von ${ }^{57} \mathrm{FeCl}_{2} \mathrm{mit}$ $\mathrm{BnMgCl}$ (4.0 Äq.) in THF ( $T=80 \mathrm{~K}) ; \delta$ (blau) $=0.27 \mathrm{~mm} \mathrm{~s}^{-1}, \Delta E_{\mathrm{Q}}$ (blau) $=1.05 \mathrm{~mm} \mathrm{~s}^{-1}$, rel. Intensität (blau) $=44 \%$; $\delta($ rot $)=0.26 \mathrm{~mm} \mathrm{~s}^{-1}, \quad \Delta E_{\mathrm{Q}}($ rot $)=1.54 \mathrm{~mm} \mathrm{~s}^{-1}, \quad$ rel. Intensität $\quad($ rot $)=43 \% ; \quad \delta$ (grün) $=1.04 \mathrm{~mm} \mathrm{~s}^{-1}$, $\Delta E_{\mathrm{Q}}$ (grün) $=1.89 \mathrm{~mm} \mathrm{~s}^{-1}$, rel. Intensität (grün) $=13 \%$. 


\subsubsection{Transmetallierung von $\mathrm{Fe}(\mathrm{acac})_{3}$ mit $\mathrm{Me}_{3} \mathrm{SiCH}_{2} \mathrm{MgCl}$}

Zuletzt rückte die Transmetallierung von $\mathrm{Fe}(\mathrm{acac})_{3}$ mit vier Äquivalenten $\mathrm{Me}_{3} \mathrm{SiCH}_{2} \mathrm{MgCl}$ in den Fokus der ESI-massenspektrometrischen Untersuchungen ( $\rightarrow$ Abbildung 31). Hier dominierte $\left(\mathrm{Me}_{3} \mathrm{SiCH}_{2}\right)_{4} \mathrm{Fe}(\mathrm{III})^{-}$das resultierende Massenspektrum im negativen lonenmodus. Daneben waren mit $\left(\mathrm{Me}_{3} \mathrm{SiCH}_{2}\right)_{3} \mathrm{Fe}(\mathrm{II})^{-}$und $\left(\mathrm{Me}_{3} \mathrm{SiCH}_{2}\right)_{3} \mathrm{Fe}(\mathrm{III}) \mathrm{Cl}^{-}$zwei weitere einkernige Spezies in geringer Signalintensität zu erkennen. Zudem lagen $\left(\mathrm{Me}_{3} \mathrm{SiCH}_{2}\right)_{5} \mathrm{Fe}_{2} \mathrm{Cl}^{-}$und $\left(\mathrm{Me}_{3} \mathrm{SiCH}_{2}\right)_{6} \mathrm{Fe}_{2} \mathrm{Cl}^{-}$mit Eisenzentren in den durchschnittlichen Oxidationszahlen +2.5 und +3.0 in geringer Signalintensität vor. Auch dieses Organoferrat-System wurde abschließend mit Mößbauer-Spektroskopie untersucht ( $\rightarrow$ Abbildung 32). Ausgehend von einer gefrorenen Reaktionslösung von ${ }^{57} \mathrm{FeCl}_{2}$ und $\mathrm{Me}_{3} \mathrm{SiCH}_{2} \mathrm{MgCl}$ (4.0 Äq.) in THF ließ sich mit einer Isomerieverschiebung von $\delta=0.30 \mathrm{~mm} \mathrm{~s}^{-1}$ und einer Quadrupolaufspaltung von $\Delta E_{\mathrm{Q}}=1.10 \mathrm{~mm} \mathrm{~s}^{-1}$ eine Eisen(III)-Spezies im high-spin-Zustand als die das Spektrum dominierende Eisenverbindung ausmachen. Daneben waren weitere Eisenverbindungen in geringerer Signalintensität vorhanden. Somit konnte auch bei der Untersuchung der Organoferrate, gebildet aus der Transmetallierung von ${ }^{57} \mathrm{FeCl}_{2}$ oder $\mathrm{Fe}(\mathrm{acac})_{3}$ mit $\mathrm{Me}_{3} \mathrm{SiCH}_{2} \mathrm{MgCl}$, eine sehr gute Korrelation beider Messmethoden festgestellt werden, da beide auf eine klare Dominanz von Eisen(III) in Lösung hinwiesen.

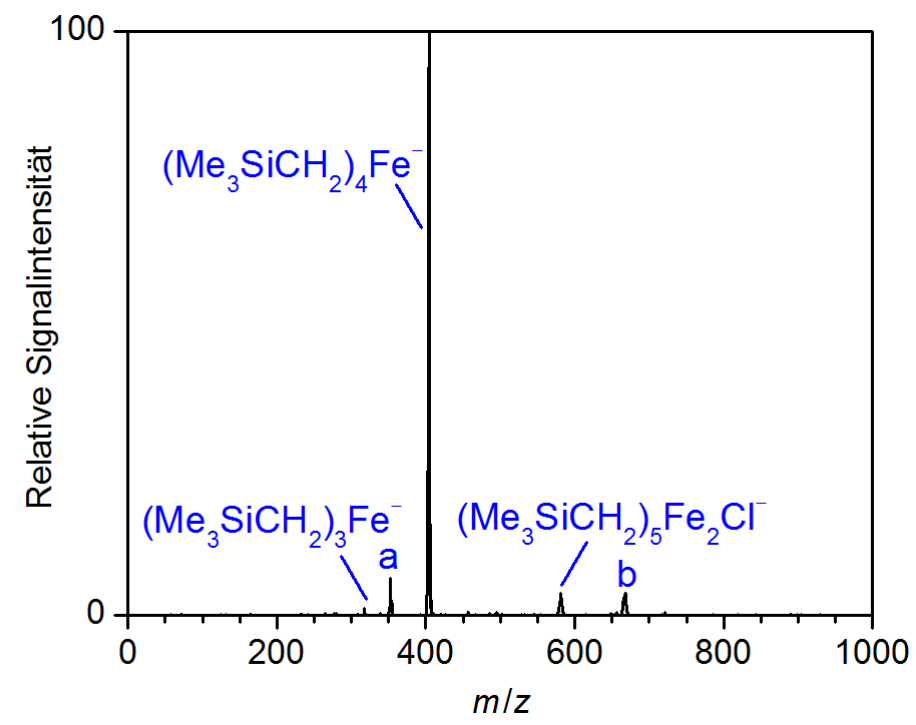

Abbildung 31. ESI-Massenspektrum (negativer lonenmodus) der Produkte aus der Reaktion von $\mathrm{Fe}(\mathrm{acac})_{3}$ (20 mM) mit $\mathrm{Me}_{3} \mathrm{SiCH}_{2} \mathrm{MgCl}$ (4.0 Äq.) in THF; $\mathrm{a}=\left(\mathrm{Me}_{3} \mathrm{SiCH}_{2}\right)_{3} \mathrm{FeCl}^{-}, \mathrm{b}=\left(\mathrm{Me}_{3} \mathrm{SiCH}_{2}\right)_{6} \mathrm{Fe}_{2} \mathrm{Cl}^{-}$. 


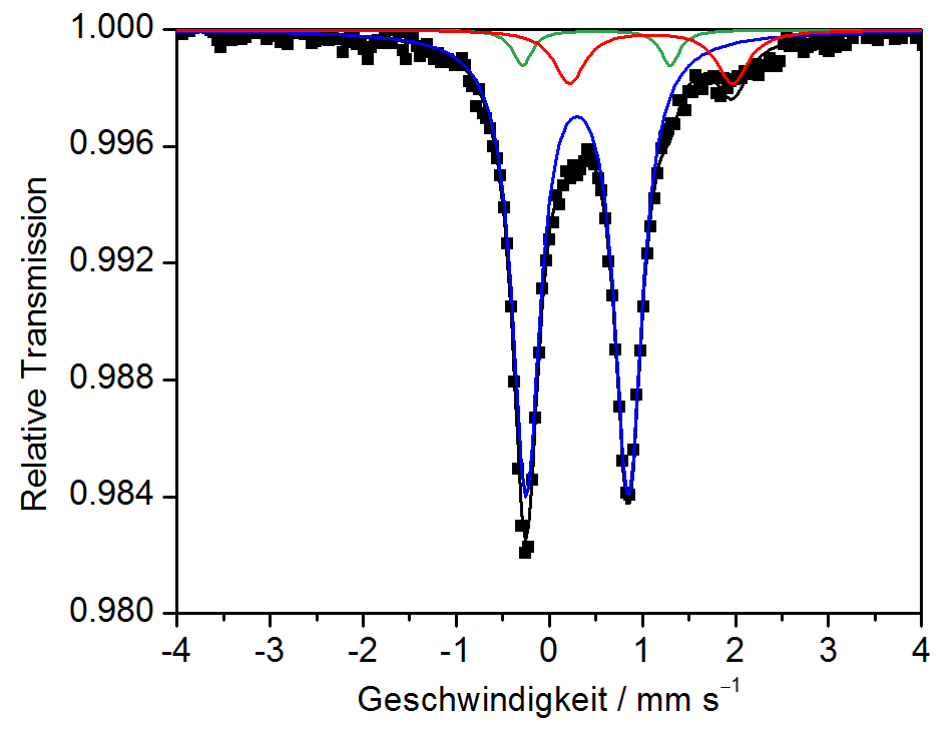

Abbildung 32. Mößbauer-Spektrum einer gefrorenen Lösung der Produkte aus der Reaktion von ${ }^{57} \mathrm{FeCl}_{2}$ mit $\mathrm{Me}_{3} \mathrm{SiCH}_{2} \mathrm{MgCl}$ (4.0 Äq.) in THF $\left(T=80 \mathrm{~K}\right.$ ); $\delta$ (blau) $=0.30 \mathrm{~mm} \mathrm{~s}^{-1}, \Delta E_{\mathrm{Q}}$ (blau) $=1.10 \mathrm{~mm} \mathrm{~s}^{-1}$, rel. Intensität $($ blau $)=84 \% ; \delta($ rot $)=1.09 \mathrm{~mm} \mathrm{~s}^{-1}, \Delta E_{\mathrm{Q}}($ rot $)=1.75 \mathrm{~mm} \mathrm{~s}^{-1}$, rel. Intensität (rot) $=11 \% ; \delta$ (grün) $=0.50 \mathrm{~mm} \mathrm{~s}^{-1}, \Delta E_{\mathrm{Q}}$ (grün) $=1.59 \mathrm{~mm} \mathrm{~s}^{-1}$, rel. Intensität (grün) $=5 \%$. 


\subsubsection{Intermolekulare Austauschreaktionen}

Um das dynamische Verhalten der Organoferrate in Lösung näher zu beschreiben, wurden verschiedene Organoferrat-Lösungen separat hergestellt und anschließend in äquimolarem Verhältnis vereint. Für alle untersuchten Systeme war ein schneller, intermolekularer Austausch organischer Substituenten charakteristisch. So war es möglich, nach Vereinigung der Probenlösungen von $\mathrm{Me}_{12} \mathrm{Fe}_{8}{ }^{-}$und seinem deuterierten Analogon $\left(\mathrm{CD}_{3}\right)_{12} \mathrm{Fe}_{8}{ }^{-}$ein sich schnell einstellendes Gleichgewicht gemischter Cluster-Anionen $\mathrm{Me}_{12-n} \mathrm{Fe}_{8}\left(\mathrm{CD}_{3}\right)_{n}^{-}(n=1-8) \mathrm{zu}$ beobachten ( $\rightarrow$ Abbildung 33).

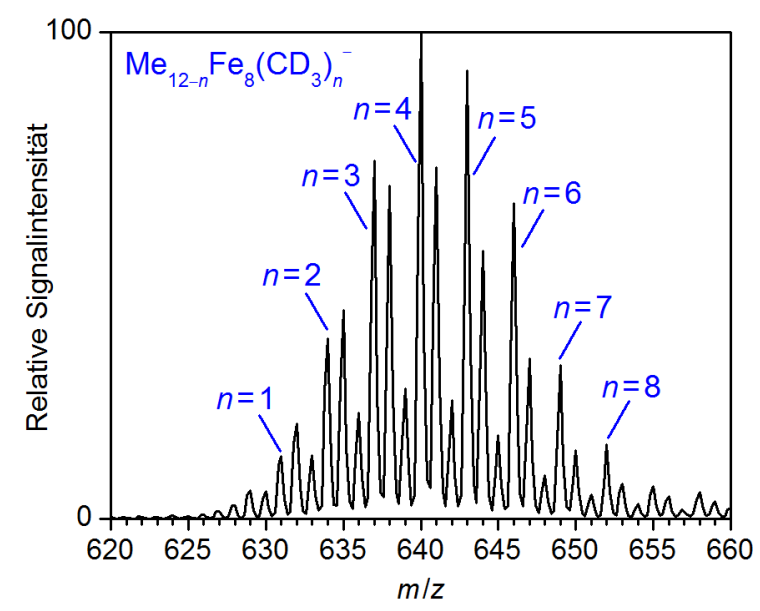

Abbildung 33. Ausschnitt aus dem ESI-Massenspektrum (negativer lonenmodus) der Produkte aus der intermolekularen Austauschreaktion zwischen $\mathrm{Fe}(\mathrm{acac})_{3} / 4 \mathrm{MeMgCl}$ und $\mathrm{Fe}(\mathrm{acac})_{3} / 4 \mathrm{CD}_{3} \mathrm{Mgl}$ in THF.

Die Bildung solcher achtkernigen Organoferrate war nicht nur auf Methyl-Gruppen beschränkt. So konnten durch äquimolare Vereinigung von $\mathrm{Me}_{12} \mathrm{Fe}_{8}{ }^{-}$mit Phenylferraten, erzeugt über Transmetallierung von $\mathrm{Fe}(\mathrm{acac})_{3}$ mit $\mathrm{PhMgCl}$ (4.0 Äq.) in $\mathrm{THF}$, bis zu fünf PhenylSubstituenten in das achtkernige Aggregat eingebaut werden ( $\rightarrow$ Abbildung 34). Daneben wurde für eine Vielzahl weiterer Systeme der intermolekulare Austausch organischer Substituenten in einkernigen Organoferraten mit Eisenzentren in den Oxidationsstufen +II und +III realisiert. Repräsentativ für solch einen Austausch soll die Vereinigung der Probenlösungen von Octyl- und Decylferraten stehen, die es ermöglichte, die vollständige Eisen(III)-Serie Oct ${ }_{4-n} \mathrm{FeDec}_{n}{ }^{-}(n=0-4)$ im resultierenden ESI-Massenspektrum im negativen Ionenmodus zu visualisieren ( $\rightarrow$ Abbildung 35). 


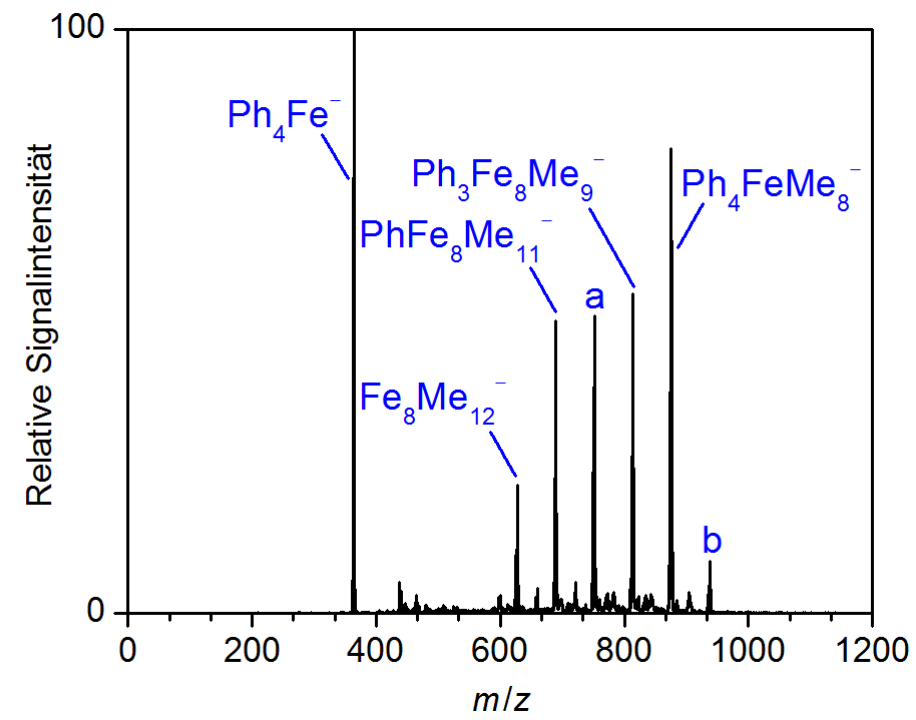

Abbildung 34. ESI-Massenspektrum (negativer lonenmodus) der Produkte aus der intermolekularen Austauschreaktion zwischen $\mathrm{Fe}(\mathrm{acac})_{3} / 4 \mathrm{PhMgCl}$ und $\mathrm{Fe}(\mathrm{acac})_{3} / 4 \mathrm{MeMgCl}$ in $\mathrm{THF}$ (trap drive 60); $\mathrm{a}=\mathrm{Ph}_{2} \mathrm{Fe}_{8} \mathrm{Me}_{10}{ }^{-}, \mathrm{b}=\mathrm{Ph}_{5} \mathrm{Fe}_{8} \mathrm{Me}_{7}^{-}$.

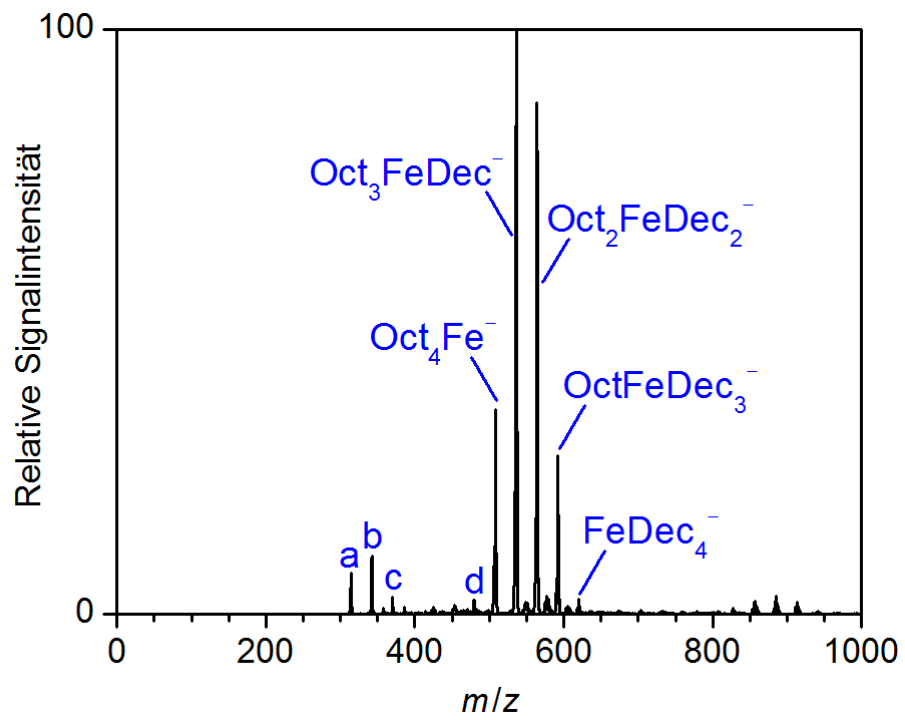

Abbildung 35. ESI-Massenspektrum (negativer lonenmodus) der Produkte aus der intermolekularen Austauschreaktion zwischen $\mathrm{Fe}(\mathrm{acac})_{3} / 4 \mathrm{OctMgCl}$ und $\mathrm{Fe}(\mathrm{acac})_{3} / 4 \mathrm{DecMgCl}$ in $\mathrm{THF} ; \mathrm{a}=\left[\mathrm{Oct}_{2}, \mathrm{Fe}, \mathrm{O}_{2}\right]^{-}$, $\mathrm{b}=\left[\mathrm{Oct}, \mathrm{Fe}, \mathrm{Dec}, \mathrm{O}_{2}\right]^{-}, \mathrm{c}=\left[\mathrm{Fe}, \mathrm{Dec}_{2}, \mathrm{O}_{2}\right]^{-}, \mathrm{d}=\mathrm{FeDec}_{3}{ }^{-}$. 


\subsubsection{Transmetallierung von $\mathrm{FeCl}_{2}$ oder $\mathrm{Fe}(\mathrm{acac})_{3}$ mit $\mathrm{RLi}$}

Um den Einfluss des Transmetallierungsreagenzes genauer zu untersuchen, wurden mit $\mathrm{FeCl}_{2}$ oder $\mathrm{Fe}(\mathrm{acac})_{3}$ je ein Eisen(II)- und ein Eisen(III)-Vorläufer zusätzlich mit OrganolithiumReagenzien RLi ( $\left.\mathrm{R}=\mathrm{Ph}, \mathrm{Mes}, \mathrm{Me}, \mathrm{Bu}, \mathrm{Me}_{3} \mathrm{SiCH}_{2}\right)$ umgesetzt. Der Wechsel von Grignard- auf Organolithium-Reagenz als Transmetallierungsmittel führte zu qualitativ vergleichbaren Ergebnissen, wobei allgemein Aggregationseffekte eine geringere Rolle spielten. Hervorzuheben war die Transmetallierung mit Methyllithium, die in ein ESI-Massenspektrum im negativen lonenmodus resultierte, das von der heterobimetallischen Eisen(III)-Spezies $\mathrm{Me}_{8} \mathrm{LiFe}_{2}{ }^{-}$dominiert wurde ( $\rightarrow$ Abbildung 36). Daneben ließen sich in Spuren erneut die einkernigen Methylferrate $\mathrm{Me}_{3} \mathrm{Fe}(\mathrm{II})^{-}$und $\mathrm{Me}_{4} \mathrm{Fe}(\mathrm{III})^{-}$nachweisen. Zusätzlich waren $\mathrm{Me}_{7} \mathrm{LiFe}_{2}{ }^{-}$ mit Eisen in der durchschnittlichen Oxidationsstufe +2.5 und Hydrolyse- und Oxidationsprodukte in geringer Signalintensität identifizierbar. Auch hier zeigte sich im Vergleich zur Transmetallierung mit $\mathrm{MeMgCl}$ eine geringere Aggregation. Die Spektren im positiven lonenmodus waren allesamt geprägt durch Spezies der allgemeinen Form $\mathrm{Li}_{m+1}(\mathrm{acac})_{m^{+}}(m=2-6)$, die zum Teil als THF-Addukte vorlagen. Wie in den vorangegangen Kapiteln bereits nachgewiesen, zeigte sich erneut, dass die Oxidationsstufe und die Natur des Eisenvorläufers keinen wesentlichen Einfluss auf die Bildung der Organoferrate hatten.

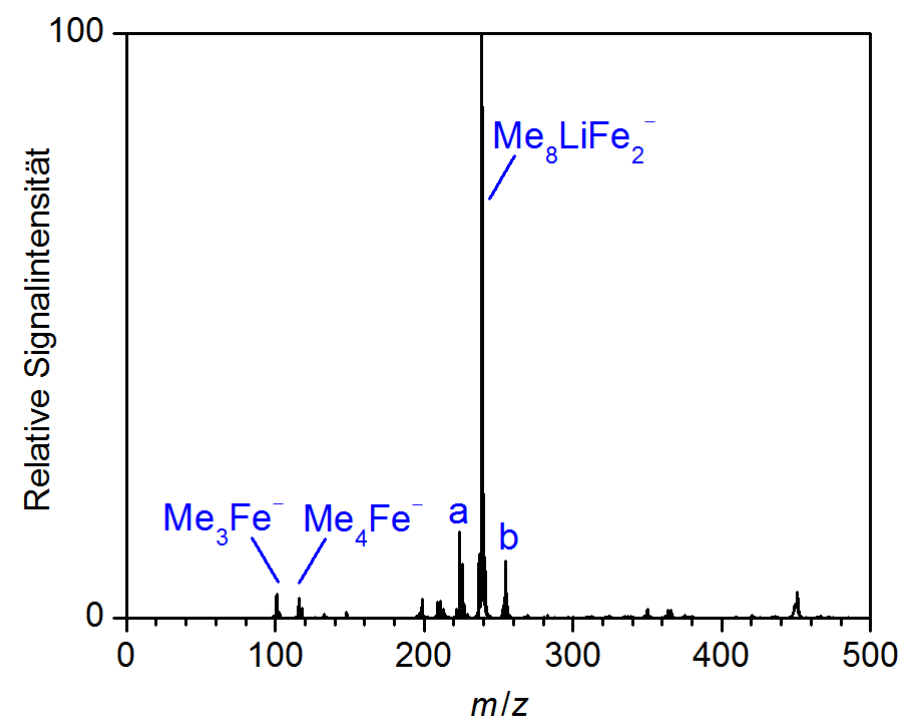

Abbildung 36. ESI-Massenspektrum (negativer lonenmodus) der Produkte aus der Reaktion von $\mathrm{Fe}(\mathrm{acac})_{3}$ (20 mM) mit MeLi (4.0 Äq.) in THF; $a=\mathrm{Me}_{7} \mathrm{LiFe}_{2}^{-}+\mathrm{Me}_{6} \mathrm{LiFe}_{2}(\mathrm{OH})^{-}, \mathrm{b}=\left[\mathrm{Me}_{8}, \mathrm{Li}, \mathrm{Fe}_{2}, \mathrm{O}\right]^{-}$. 


\subsubsection{Transmetallierung von $\mathrm{FeCl}_{2}$ oder $\mathrm{Fe}(\mathrm{acac})_{3}$ mit $\mathrm{R}_{2} \mathrm{Zn}$}

Als weitere Vergleichsmöglichkeit dienten die Transmetallierungsreaktionen von $\mathrm{FeCl}_{2}$ oder $\mathrm{Fe}(\mathrm{acac})_{3}$ mit den kommerziell erwerbbaren Organozink-Reagenzien $\mathrm{Ph}_{2} \mathrm{Zn}$ und $\mathrm{Et}_{2} \mathrm{Zn}$. Zunächst wurde $\mathrm{Fe}(\mathrm{acac})_{3}$ mit jeweils vier Äquivalenten an Diphenylzink oder Diethylzink umgesetzt. Weder Anionen noch Kationen waren nach Ablauf dieser Reaktion zu beobachten. Ausgehend von $\mathrm{FeCl}_{2}$ ließen sich nach Reaktion mit $\mathrm{R}_{2} \mathrm{Zn}$ ( $\mathrm{R}=\mathrm{Ph}$, Et; 4.0 Äq.) vor allem Zn-haltige Kationen des Typs EtZn(THF $)_{n}{ }^{+}(n=2,3)$ oder $\operatorname{Ph}_{2} \mathrm{Zn}_{2} \mathrm{Cl}(\mathrm{THF})_{n}{ }^{+}(n=2,3)$ detektieren. Es blieb festzuhalten, dass eisenhaltige Spezies in Abwesenheit von Additiven oder Liganden ausschließlich als Neutralspezies vorlagen und deren Detektion mittels ESIMassenspektrometrie nicht möglich war. Auch ein Variieren der Reaktionszeiten ( $t=1 \mathrm{~min}$, 30 min, 2 h, 24 h) und der Reaktionstemperaturen ( $T=195 \mathrm{~K}, 273 \mathrm{~K}, 298 \mathrm{~K}$ ) änderte nichts an diesem Sachverhalt. 


\subsubsection{Bildung und Stabilität von Organoferraten}

Es konnte gezeigt werden, dass das erste Äquivalent an Grignard-Reagenz vornehmlich die Reduktion des eingesetzten Eisen(III)-Präkatalysators bewirkte. Dies ist im Einklang mit der Literatur ${ }^{[35 f, 39 a-d, 77]}$ und bestätigt die reduktive Wirkung der Grignard-Reagenzien auf die entsprechenden Eisenvorläufer ( $\rightarrow$ Schema 5 ).

$$
\begin{aligned}
& + \text { III } \quad+\text { II } \\
& 2 \mathrm{FeL}_{3}+2 \mathrm{RMgX} \longrightarrow 2 \mathrm{FeL}_{2}+\mathrm{R}_{2}+2 \mathrm{LMgX}
\end{aligned}
$$

Schema 5. Allgemeine Reaktionsgleichung für die Zwei-Elektronen-Reduktion von $2 \mathrm{Fe}(\mathrm{III}) \mathrm{zu} 2 \mathrm{Fe}$ (II) durch Grignard-Reagenzien RMgX unter Freisetzung des Homokupplungsproduktes $\mathrm{R}_{2}$.

Die Natur des Eisenvorläufers und dessen Oxidationsstufe hat keinen entscheidenden Einfluss auf die Bildung der Organoferrate. Besonders hervorzuheben ist, dass die Transmetallierung nicht auf Stufe der entsprechenden Neutral-Spezies stehen bleibt, sondern ein weiterer organischer Rest übertragen wird und sich organische Ferrat-Komplexe bilden. Das Vorliegen der Organoferrate in den jeweiligen Oxidationsstufen konnte durch Mößbauer-Experimente bestätigt werden. Eine sehr gute Korrelation dieser Messmethode mit ESIMassenspektrometrie zeigt zudem, dass Neutralteilchen in Abwesenheit von Additiven und Liganden keine entscheidende Rolle spielen. Mit $\mathrm{Ph}_{6} \mathrm{MgFe}^{-}, \mathrm{Ph}_{5} \mathrm{MgFeCl}^{-}$sowie $\mathrm{Me}_{8} \mathrm{LiFe}_{2}{ }^{-}$ konnten einige heterobimetallische Spezies detektiert werden. Für artverwandte, heterobimetallische Cuprate konnte in der Vergangenheit bereits gezeigt werden, dass das Lithium über die organischen Reste koordiniert und damit eine verbrückende Funktion hat. ${ }^{[55 c]}$ Ähnliche Strukturen sind für die heterobimetallischen Ferrat-Komplexe anzunehmen $\left(\rightarrow\right.$ Schema 6). Denkbar ist auch, dass ursprünglich eine dianionische Spezies $\mathrm{Ph}_{5} \mathrm{Fe}^{2-}$ mit Eisen(III)-Zentrum in Lösung vorlag. Nach Anlagerung eines MgX-Kations ( $\mathrm{X}=\mathrm{Cl}$ oder $\mathrm{Ph}$ ) wären ebenfalls Anionen der Form $\mathrm{Ph}_{6} \mathrm{MgFe}^{-}$und $\mathrm{Ph}_{5} \mathrm{MgFeCl}^{-}$detektierbar.

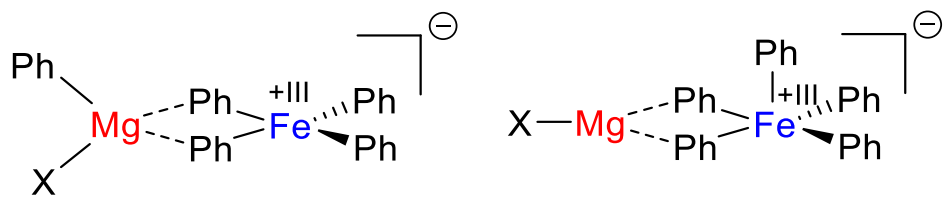<smiles>CC(C)[Al](C)[Ge](C)(C)C</smiles>

Schema 6. Strukturvorschläge für die heterobimetallischen Spezies $\mathrm{Ph}_{5} \mathrm{MgFeX}^{-}(\mathrm{X}=\mathrm{Cl}, \mathrm{Ph})$ und $\mathrm{Me}_{8} \mathrm{LiFe}_{2}{ }^{-}$.

Die hohe Tendenz von Eisen(III), organische Reste anzulagern und anionische Komplexe zu bilden, spiegelt dessen hohe Lewis-Acidität wider. Im Gegensatz dazu formt Magnesium als 
eher elektropositives Element vor allem kationische Spezies. ${ }^{[78]}$ Analog dazu zeichnet sich Lithium durch seinen in Relation zu Eisen elektropositiven Charakter aus. Auch hier ist die Separation von Eisen und Lithium in anionische und kationische Spezies sehr deutlich zu sehen.

Die Organoferrate, die in Abwesenheit von Additiven und Liganden mittels ESI-Massenspektrometrie nachgewiesen werden konnten, tragen im Wesentlichen EisenKerne in den Oxidationsstufen +II und +III. Daneben lassen sich Eisen(IV)-Spezies und mehrkernige Aggregate mit durchschnittlichen Oxidationszahlen $<+l l$ detektieren. Etliche dieser Organoferrate konnten durch Mößbauer-Spektroskopie bestätigt werden. Zusätzlich können durch diese Analysemethode Aussagen über den Spinzustand beobachteter Eisenspezies getroffen werden. So weisen Anionen mit Eisen in der Oxidationsstufe +ll tendenziell eher einen low-spin-Charakter auf, wohingegen sämtliche Organoferrate mit Eisen(III)-Kern im high-spin-Zustand vorliegen. Unabhängig davon, ob Grignard- oder Organolithium-Reagenzien als Transmetallierungsmittel eingesetzt worden sind, ist die Bildung der Organoferrate nur wenig beeinflusst. Einzige Ausnahme stellt die Transmetallierung mit Methyllithium dar. Hier ist deutlich eine geringere Aggregation zu beobachten. Andere Transmetallierungssysteme $\left(\mathrm{Ph}, \mathrm{Me}_{3} \mathrm{SiCH}_{2}\right)$ zeigen eine weniger ausgeprägte, aber trotzdem vorhandene Aggregationstendenz. Die höhere Reaktivität der Organolithium-Reagenzien durch eine stärker polarisierte Bindung zwischen Metall und Kohlenstoff scheint eine Bildung höherer Aggregate maßgeblich einzuschränken. In Relation dazu zeichnen sich die Bindungen von Zink und Kohlenstoff in zinkorganischen Reagenzien durch einen stärker kovalenten Bindungscharakter aus. ${ }^{[79]}$ Die dadurch herabgesenkte Reaktivität scheint Ursache dafür zu sein, dass bei der Transmetallierung mit OrganozinkReagenzien die gebildeten Organoeisen-Spezies auf Stufe der Neutralteilchen stehenbleiben. Überraschend ist die oft hohe Dominanz der Eisen(III)-At-Komplexe unter reduzierenden Bedingungen. Selbst wenn die Transmetallierung direkt von Eisen(II)-Vorläufern ausgehend erfolgte, änderte dies nichts an der Dominanz dieser Anionen. Das Vorhandensein von größeren Mengen an Sauerstoff, die die Anwesenheit von Eisen(III)-At-Komplexen erklären würde, kann ausgeschlossen werden, da niedervalente und als sehr sauerstoffempfindlich geltende Eisen-Spezies wie $\mathrm{Me}_{12} \mathrm{Fe}_{8}{ }^{-}$erfolgreich detektiert werden konnten. ${ }^{[39 g]}$ Auch andere Arbeiten zeigen die Präsenz Eisen(III)-haltiger At-Komplexe in Lösung ${ }^{[39,, f, 77]}$ und erklären diese durch das Auftreten von Redoxdisproportionierungen ( $\rightarrow$ Schema 7). ${ }^{[39 b, 77]}$ Auch die Bildung von Anionen mit Eisen in der Oxidationsstufe +IV kann über eine analoge Reaktion erklärt werden. ${ }^{[80]}$ 

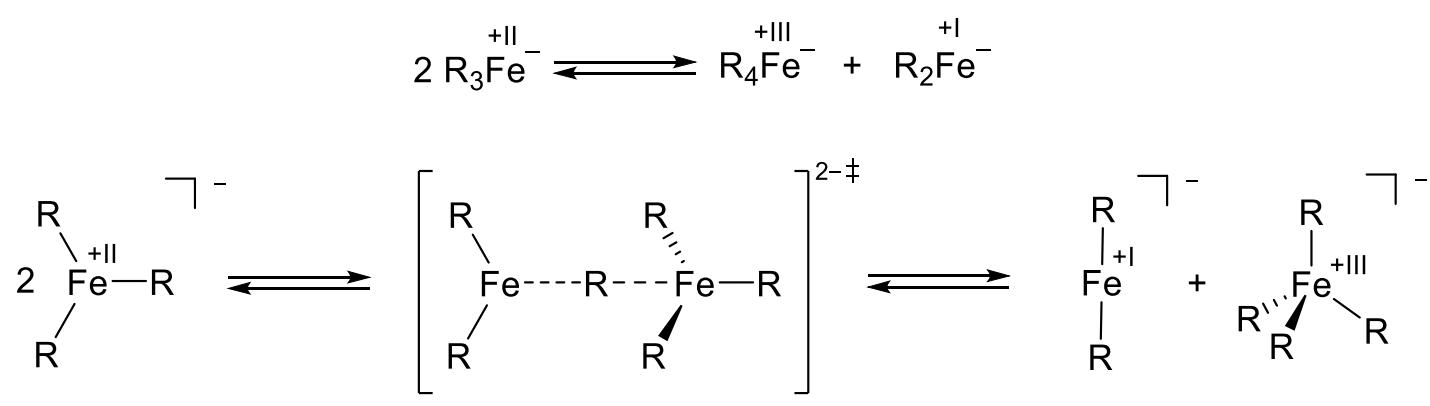

Schema 7. Redoxdisproportionierung der Eisen(II)-Spezies $\mathrm{R}_{3} \mathrm{Fe}^{-}$als mögliche Erklärung der Bildung von $\mathrm{R}_{4} \mathrm{Fe}(\mathrm{III})^{-}$unter reduzierenden Bedingungen. Die räumliche Nähe der Eisenzentren muss gewährleistet sein, damit diese Reaktion stattfinden kann.

Bedenkt man das dynamische Verhalten der Organoferrate in Lösung, welches sich durch sehr schnell ablaufende, intermolekulare Austauschreaktionen ausdrückte, so scheint eine Redoxdisproportionierung durchaus wahrscheinlich. So kann $\mathrm{R}_{3} \mathrm{Fe}(\mathrm{II})^{-}$durch Disproportionierung die entsprechende Eisen(III)-Spezies $\mathrm{R}_{4} \mathrm{Fe}^{-}$bilden. Im Zuge dieser Reaktion sollte auch eine niedervalente Eisen(I)-Spezies $\mathrm{R}_{2} \mathrm{Fe}^{-}$entstehen. Aus den bisher gezeigten Ergebnissen geht die Anwesenheit einer solchen Spezies nicht hervor. Auf eine detaillierte Diskussion über die Anwesenheit niedervalenter Eisenspezies und deren Rolle während der Transmetallierung soll im späteren Verlauf dieser Arbeit ein besonderes Augenmerk gerichtet werden (siehe Kapitel 4.1.2.8).

Damit die Disproportionierung von $\mathrm{R}_{3} \mathrm{Fe}(\mathrm{II})^{-}$und der damit einhergehende Elektronentransfer stattfinden können, muss die räumliche Nähe der beiden Eisenzentren gewährleistet sein ( $\rightarrow$ Schema 7). Sterisch anspruchsvolle organische Reste halten die beiden Eisenzentren voneinander fern und die Disproportionierung ist nicht oder nur schwer möglich. Dies kann als Grund für die Dominanz von $\mathrm{Mes}_{3} \mathrm{Fe}(\mathrm{II})^{-}$angesehen werden, da dieser At-Komplex sterisch sehr anspruchsvolle Mesitylsubstituenten aufweist. Auch für anionische Eisenspezies, die ortho-subsituierte aromatische Substituenten tragen, ist eine Dominanz von Eisen(II)Komplexen zu beobachten. Die entsprechenden para-substituierten aromatischen Substituenten wiederum lassen organische Eisen(III)-Spezies wieder mehr in den Vordergrund treten ( $\rightarrow$ Vgl. Abbildung 20 und 23, Kapitel 4.1.1.3). Die Sterik der organischen Substituenten hat somit einen entscheidenden Einfluss darauf, welche Eisen-Spezies gebildet werden und in welchem Maß sie in Lösung auftreten. Mit abnehmendem sterischen Anspruch der organischen Substituenten steigt zudem der Aggregationsgrad. Treten bei Benzyl- und Phenylferraten hohe Aggregate nur in geringen Spuren auf, so ist für Methylsubstituenten als sterisch wenig anspruchsvollem Rest die Bildung hoher Aggregate favorisiert. Betrachtet man 
den Aggregationsgrad im Zusammenhang mit der elektrischen Leitfähigkeit, so lässt sich eine klare Tendenz ablesen ( $\rightarrow$ Tabelle 5 ). Je höher der Aggregationsgrad des Transmetallierungssystems, desto geringerer die spezifische elektrische Leitfähigkeit $\kappa$.

Tabelle 5. Spezifische elektrische Leitfähigkeit $\kappa / \mu \mathrm{S} \mathrm{cm}^{-1}$ der Organoferrate, entstanden durch Transmetallierung von $\mathrm{Fe}(\mathrm{acac})_{3}(50 \mathrm{~mm})$ mit Grignard-Reagenzien in THF bei $T=273 \mathrm{~K} ; \mathrm{ArF}_{\mathrm{F}}=3,5-\left(\mathrm{CF}_{3}\right)_{2}-\mathrm{C}_{6} \mathrm{H}_{3}$.

\begin{tabular}{lc}
\hline \multicolumn{1}{c}{ Probenlösung in THF } & $\boldsymbol{\kappa} / \boldsymbol{\mu} \mathbf{~ c m}^{-1}$ \\
\hline $\mathrm{Fe}(\mathrm{acac})_{3}+4 \mathrm{PhMgCl}$ & $99 \pm 16$ \\
$\mathrm{Fe}(\mathrm{acac})_{3}+4 \mathrm{MesMgBr}$ & $339 \pm 6$ \\
$\mathrm{Fe}(\mathrm{acac})_{3}+4\left(\mathrm{Ar}_{\mathrm{F}}\right) \mathrm{MgCl}$ & $450 \pm 6$ \\
$\mathrm{Fe}(\mathrm{acac})_{3}+4 \mathrm{MeMgCl}$ & $65 \pm 17$ \\
$\mathrm{Fe}(\mathrm{acac})_{3}+4 \mathrm{BnMgCl}$ & $201 \pm 10$ \\
$\mathrm{Fe}(\mathrm{acac})_{3}+4 \mathrm{Me}_{3} \mathrm{SiCH}_{2} \mathrm{MgCl}$ & $319 \pm 24$ \\
\hline
\end{tabular}

Für Alkylferrate, die $\beta$-H-Atome aufweisen, kann mit steigender Anzahl an Methyleneinheiten in der Alkylkette eine höhere Stabilität beobachtet werden. So können durch Transmetallierung mit EtMgCl und BuMgCl keine Alkylferrate in Abwesenheit von Additiven und Liganden erzeugt werden, wohingegen die höheren Homologen der aliphatischen Grignard-Reagenzien, zumindest kurzzeitig stabile Organoferrate aus $\mathrm{Fe}(\mathrm{acac})_{3}$ erzeugen können. Ganz allgemein kann die geringe Stabilität der Alkylferrate mit der Anwesenheit von $\beta$-H-Atomen erklärt werden, die einen Zerfall der anionischen Spezies über $\beta$-H-Eliminierung möglich machen. Besonders markant sollte sich dieser Effekt auf das Ethyl-System auswirken, da hier im Vergleich mit den höheren Homologen ein zusätzliches $\beta$-H-Atom vorhanden ist. Zusätzlich erklärt ein entropischer Effekt die geringe Stabilität methylenarmer Alkylferrate. So sind mit Decan und Decen mögliche Zerfallsprodukte der Decylferrate bei Umgebungstemperatur flüssig, wohingegen Ethan und Ethen sowie Butan und Buten bei analogen Bedingungen als Gas die Reaktionslösung verlassen können. Entropisch besonders günstig ist die Bildung von Gasen aus Flüssigkeiten, da diese die Entropie des Systems erhöhen.

Für eine Vielzahl an Transmetallierungssystemen können Eisen-Spezies der allgemeinen Form $\left[\mathrm{R}, \mathrm{Fe}, \mathrm{O}_{2}\right]^{-}$beobachtet werden, die durch eine bimolekulare Reaktion mit molekularem Sauerstoff entstanden sind. Dieser ist in Spuren im Hochvakuumsystem des Massenspektrometers vorhanden. Mehrere Strukturen sind für diese Anionen denkbar ( $\rightarrow$ Schema 8 ). So können beide Sauerstoff-Atome als Oxid an das Eisenzentrum binden (A). Auch kann nur ein 
Sauerstoff-Atom oxidisch an Eisen gebunden vorliegen, wohingegen das andere in die MetallKohlenstoff-Bindung insertiert (B). In diesen Fällen lässt sich dem Eisenzentrum formal die Oxidationsstufe +IV bzw. +II zuordnen. Möglich wäre auch, dass Sauerstoff als Peroxid an Eisen bindet $(C)$ oder peroxidisch in die Eisen-Kohlenstoff-Bindung insertiert (D), wodurch elektronenreiche, niedervalente Eisen-Spezies vorliegen würden. Aufgrund der hypothetischen Oxidationsstufen der Eisen-Zentren scheint die Eisen(II)-Spezies am wahrscheinlichsten (B), da Organoferrate in den Oxidationsstufen $0,+\mathrm{I}$ und +IV nicht oder nur in Einzelfällen auftraten. Zudem belegen DFT-Rechnungen aus der Literatur, dass Sauerstoffinsertionen speziell bei organischen Eisen(II)-Verbindungen auftreten können. ${ }^{[81]}$ In wenigen Fällen sind zusätzlich Anionen des Typs $\left[\mathrm{R}_{2}, \mathrm{Fe}, \mathrm{O}_{2}\right]^{-}$und $\left[\mathrm{R}_{4}, \mathrm{Fe}, \mathrm{O}_{2}\right]^{-}$detektierbar. Auch hier sind Insertionsreaktionen von Sauerstoff in die Metall-Kohlenstoff-Bindung möglich. Gerade in letzterem Fall ist eine Insertionsreaktion von Sauerstoff sogar sehr wahrscheinlich, da anderenfalls sehr hochvalente und damit wenig wahrscheinliche Oxidationsstufen von Eisen vorliegen würden.<smiles></smiles>

A<smiles>[R]O[13C](=O)[13CH]=O</smiles>

B<smiles>[R][Fe][O+](C)OO</smiles>

C<smiles>[R]OO[Pb]C(C)C</smiles>

D

Schema 8. Mögliche Strukturen für die sauerstoffhaltigen Spezies der allgemeinen Form $\left[\mathrm{R}, \mathrm{Fe}, \mathrm{O}_{2}\right]^{-}$. 


\subsubsection{Transmetallierung in Anwesenheit von Additiven und Liganden}

\subsubsection{Transmetallierung von $\mathrm{Fe}(\mathrm{acac})_{3}$ mit $\mathrm{ArMgX}$ in Anwesenheit von TMEDA}

TMEDA, als eines der gängigsten Additive für Eisen-katalysierte Kreuzkupplungsreaktionen (siehe Kapitel 1.2), wurde verschiedenen Transmetallierungssystemen zugesetzt, um seinen Einfluss auf die Bildung und die Stabilität der Organoferrate näher zu beleuchten. Die Transmetallierung von $\mathrm{Fe}(\mathrm{acac})_{3}$ mit $\mathrm{PhMgCl}$ (4.0 Äq.) in Anwesenheit von TMEDA (4.0 Äq.) wies im Wesentlichen zwei Veränderungen auf ( $\rightarrow$ Abbildung 37). Zum einen ist das vierkernige Aggregat $\mathrm{Ph}_{7} \mathrm{Fe}_{4}{ }^{-}$im hohen Massenbereich nicht länger zu beobachten. Zum anderen war eine deutlich erhöhte Signalintensität und zeitliche Stabilität von $\mathrm{Ph}_{3} \mathrm{Fe}(\mathrm{II})^{-}$ auffällig. So lässt sich grafisch aus einer kinetischen Betrachtung für $\mathrm{Ph}_{3} \mathrm{Fe}(\mathrm{II})^{-}$eine Halbwertszeit von $t_{1 / 2}=580 \pm 60 \mathrm{~s}$ bestimmen. Damit liegt die beobachtete Halbwertszeit dieser Spezies in Anwesenheit von TMEDA deutlich über jener, die ohne die Präsenz des Additivs ermittelt werden konnte (siehe 4.1.1.1, $t_{1 / 2}=50 \pm 15 \mathrm{~s}$ ). Festzuhalten bleibt zudem, dass keine Spezies aus TMEDA und Eisen nachgewiesen wurden. Vielmehr konnte die Inkorporation dieses Additivs in Mg-haltige Kationen des Typs $\mathrm{Mg}_{2}(\operatorname{acac})_{2} \mathrm{X}(\mathrm{TMEDA})_{n}(\mathrm{X}=\mathrm{Cl}$, $\mathrm{Br} ; n=1$, 2) festgestellt werden. Mit einer zeitlich stabilen Leitfähigkeit von $83 \pm 5 \mu \mathrm{sm}^{-1}$ zeigte die Probenlösung einen nur unwesentlich niedrigeren Wert in Relation zu der analogen Reaktionslösung in Abwesenheit von TMEDA (siehe 4.1.1.1, $\kappa=99 \pm 16 \mu \mathrm{S} \mathrm{cm}^{-1}$ ).

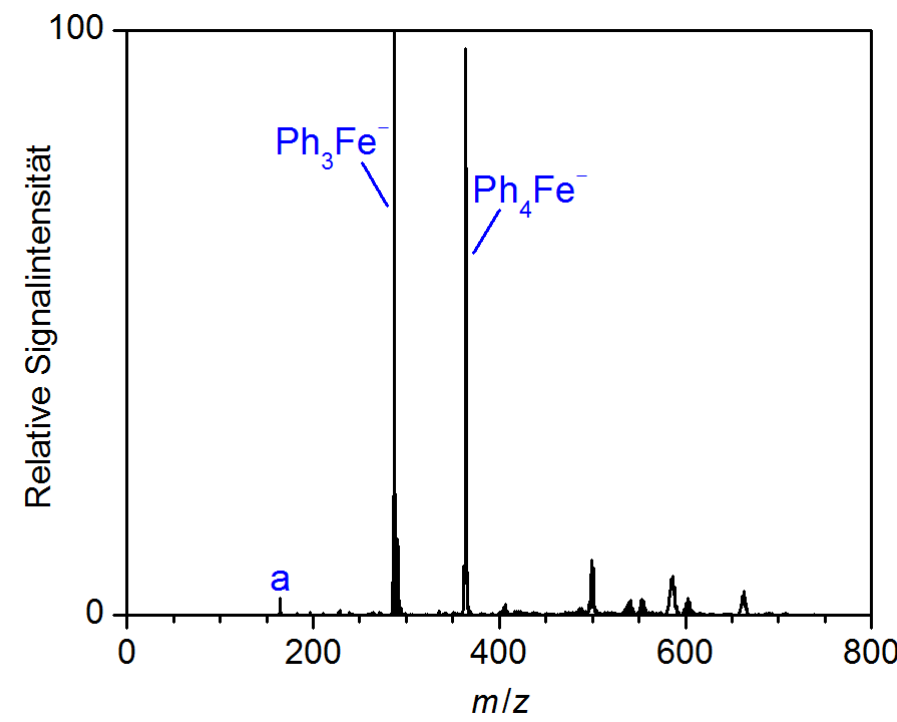

Abbildung 37. ESI-Massenspektrum (negativer lonenmodus) der Produkte aus der Reaktion von $\mathrm{Fe}(\mathrm{acac})_{3}$ (20 mM) mit TMEDA (4.0 Äq.) und PhMgCl (4.0 Äq.) in THF; $\mathrm{a}=\left[\mathrm{Ph}, \mathrm{Fe}, \mathrm{O}_{2}\right]^{-}$. 
Auch das resultierende Mößbauer-Spektrum einer Reaktionslösung von ${ }^{57} \mathrm{FeCl}_{2}$ mit $\mathrm{PhMgCl}$ (4.0 Äq.) wies in Anwesenheit von TMEDA (4.0 Äq.) Veränderungen auf. War das Spektrum zuvor durch ein breites und asymmetrisches Dublett Eisen(III)-haltiger Spezies geprägt, konnte bei Präsenz von TMEDA und durch dessen stabilisierenden Einfluss auf $\mathrm{Ph}_{3} \mathrm{Fe}(\mathrm{II})^{-}$ diese Spezies nun im low-spin-Zustand detektiert werden ( $\rightarrow$ Abbildung 38). Zudem konnte eine Eisen(III)-Spezies als scharfes und symmetrisches Mößbauer-Dublett im high-spinZustand beobachtet werden. Als mögliche Ursache für die veränderten magnetischen Eigenschaften der Probe kommt die durch TMEDA induzierte strukturelle Änderung der Kationen in Frage. Hingegen zeigten Probenlösungen aus $\mathrm{Fe}(\mathrm{acac})_{3}$ und $\mathrm{RMgBr}$ (4.0 Äq., $\mathrm{R}=\mathrm{Mes}, \mathrm{Ar}_{\mathrm{F}}$ ) in Anwesenheit von TMEDA (4.0 Äq.) keine auffälligen qualitativen oder quantitativen Veränderungen.

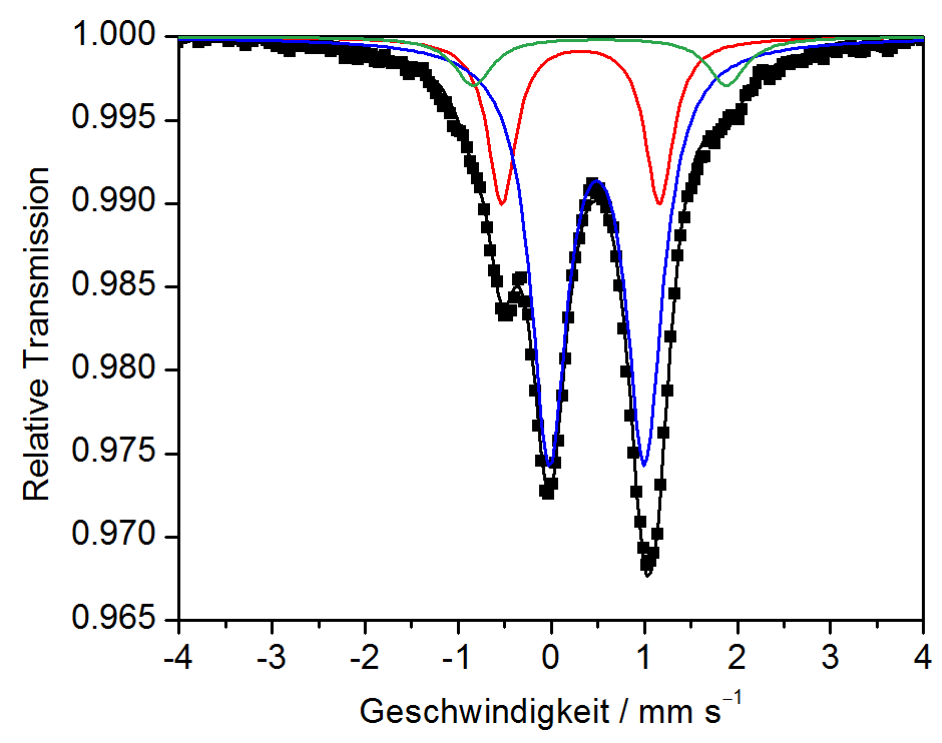

Abbildung 38. Mößbauer-Spektrum einer gefrorenen Lösung der Produkte aus der Reaktion von ${ }^{57} \mathrm{FeCl}_{2}$ mit TMEDA (4.0 Äq.) und PhMgCl (4.0 Äq.) in THF ( $T=80 \mathrm{~K}$ ); $\delta$ (blau) $=0.49 \mathrm{~mm} \mathrm{~s}^{-1}, \Delta E_{\mathrm{Q}}$ (blau) $=1.03 \mathrm{~mm} \mathrm{~s}^{-1}$, rel. Intensität $\quad($ blau $)=70 \% ; \quad \delta($ rot $)=0.32 \mathrm{~mm} \mathrm{~s}^{-1}, \quad \Delta E_{\mathrm{Q}}($ rot $)=1.69 \mathrm{~mm} \mathrm{~s}^{-1}, \quad$ rel. $\quad$ Intensität $\quad($ rot $)=22 \%$; $\delta$ (grün) $=0.52 \mathrm{~mm} \mathrm{~s}^{-1}, \Delta E_{\mathrm{Q}}($ grün $)=2.72 \mathrm{~mm} \mathrm{~s}^{-1}$, rel. Intensität (grün) $=8 \%$. 


\subsubsection{Transmetallierung von $\mathrm{Fe}(\mathrm{acac})_{3}$ mit AlkMgX in Anwesenheit von TMEDA}

Das polynukleare Anion $\mathrm{Me}_{12} \mathrm{Fe}_{8}{ }^{-}$, das nach Transmetallierung von $\mathrm{Fe}(\mathrm{acac})_{3}$ mit $\mathrm{MeMgX}$ (4.0 Äq, $\mathrm{X}=\mathrm{Cl}, \mathrm{Br}$ ) das resultierende ESI-Massenspektrum im negativen lonenmodus klar dominierte, wurde in Anwesenheit von TMEDA nicht länger gebildet ( $\rightarrow$ Abbildung 39). Vielmehr waren einkernige Methylferrate in geringer absoluter Signalintensität vorherrschend, wobei $\mathrm{Me}_{4} \mathrm{Fe}(\mathrm{III})^{-}$bei $\mathrm{m} / \mathrm{z} 116$ den Hauptpeak darstellte. Auch das entsprechende MößbauerSpektrum bestätigte diesen Sachverhalt und zeigte die alleinige Präsenz einer Eisen(III)Spezies im high-spin-Zustand ( $\rightarrow$ Abbildung 40). Dass es sich hierbei um den zuvor genannten Eisen-At-Komplex $\mathrm{Me}_{4} \mathrm{Fe}(\mathrm{III})^{-}$handelte, war zweifelhaft, da die spezifische elektrische Leitfähigkeit einer Probenlösung von Fe(acac) $)_{3}$ mit TMEDA (4.0 Äq.) und MeMgCl (4.0 Äq.) gegen null tendierte. Somit waren in Anwesenheit von TMEDA neutrale Eisen(III)Spezies in Lösung vorherrschend.

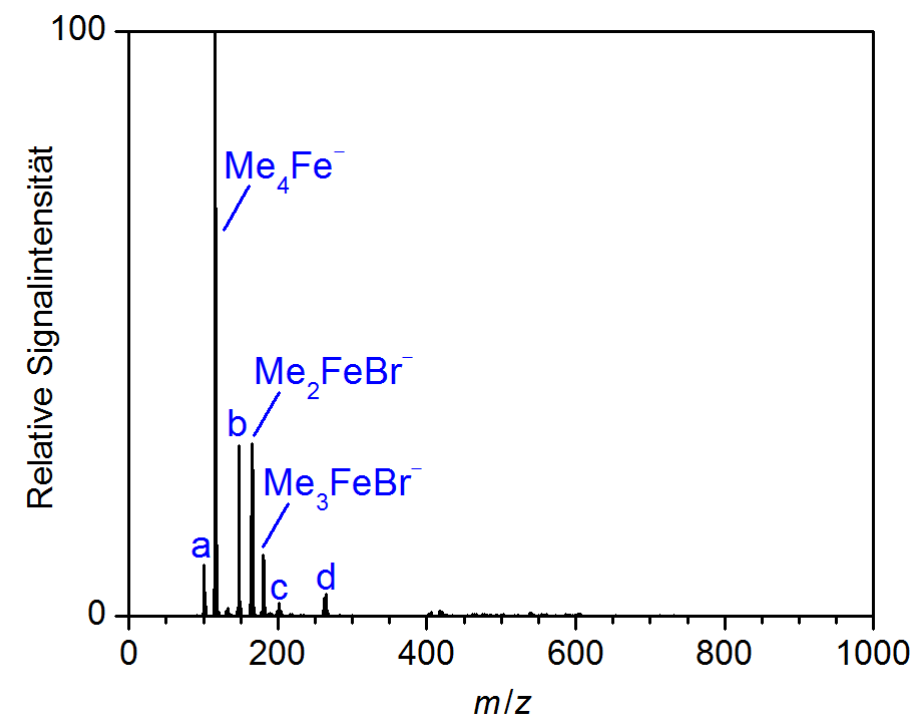

Abbildung 39. ESI-Massenspektrum (negativer lonenmodus) der Produkte aus der Reaktion von $\mathrm{Fe}(\mathrm{acac})_{3}$ (20 mM) mit TMEDA (4.0 Äq.) und $\mathrm{MeMgCl}$ (4.0 Äq.) in THF; $a=\mathrm{Me}_{3} \mathrm{Fe}^{-}, \mathrm{b}=\left[\mathrm{Me}_{4}, \mathrm{Fe}_{2} \mathrm{O}_{2}\right]^{-}, \quad \mathrm{c}=\mathrm{Me}_{6} \mathrm{Fe}_{2}{ }^{-}$, $\mathrm{d}=\mathrm{MgBr}_{3}{ }^{-}$. 


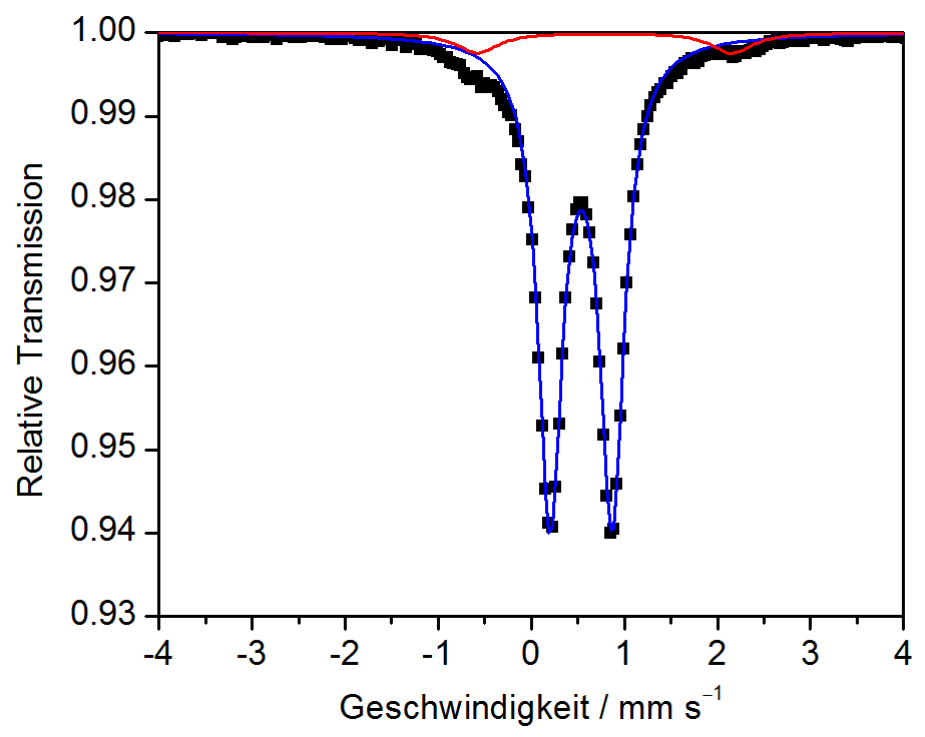

Abbildung 40. Mößbauer-Spektrum einer gefrorenen Lösung der Produkte aus der Reaktion von ${ }^{57} \mathrm{FeCl}_{2}$ mit TMEDA (4.0 Äq.) und MeMgCl (4.0 Äq.) in THF ( $T=80 \mathrm{~K}$ ); $\delta$ (blau) $=0.53 \mathrm{~mm} \mathrm{~s}^{-1}, \Delta E_{\mathrm{Q}}(\mathrm{blau})=0.67 \mathrm{~mm} \mathrm{~s}^{-1}$, rel. Intensität (blau) $=98 \% ; \delta($ rot $)=0.50 \mathrm{~mm} \mathrm{~s}^{-1}, \Delta E_{\mathrm{Q}}($ rot $)=2.22 \mathrm{~mm} \mathrm{~s}^{-1}$, rel. Intensität $($ rot $)=2 \%$.

Die alleinige Präsenz Eisen(III)-haltiger Spezies in Lösung ist überraschend, da im MößbauerExperiment angereichertes Eisen(II)-chlorid unter reduktiven Bedingungen und unter sorgfältigem Ausschluss von Sauerstoff eingesetzt wurde. Da die Mößbauer-Spektroskopie eine Methode ist, die unabhängig vom Ladungszustand die gesamte Bandbreite an Eisenverbindungen in Lösung erfasst, war dies ein klares Zeichen dafür, dass nicht alle Bestandteile der Probe in die Mößbauer-Messzelle transferiert worden waren. Um dieses Phänomen besser verstehen zu können, wurde das Experiment in einem größeren Maßstab wiederholt und auf makroskopischer Ebene betrachtet ( $\rightarrow$ Abbildung 41 ). Die ursprünglich gelbe Lösung von $\mathrm{FeCl}_{2}$ und TMEDA in THF wurde unmittelbar nach Zugabe des GrignardReagenzes dunkler, bis sie nach $t=2$ min komplett schwarz vorlag. Nachdem die schwarze Lösung entfernt wurde, verblieb ein schwarzer Niederschlag, der in den Experimenten zuvor bei niedrigen Eisenkonzentrationen nicht in relevanten Mengen in Erscheinung trat. Über eine komplexometrische Titration wurde der Eisengehalt im Feststoff und der verbliebenen Lösung nach deren vorheriger Separation bestimmt. So konnte ermittelt werden, dass $38 \pm 4 \%$ des ursprünglich eingesetzten Eisens im Feststoff vorlag, wohingegen $60 \pm 7 \%$ in Lösung verblieb. Ein Mößbauer-Experiment des Feststoffes führte zu einem wenig charakteristischen Mößbauer-Dublett $\left(\delta=0.26 \mathrm{~mm} \mathrm{~s}^{-1}, \Delta E_{\mathrm{Q}}=0.76 \mathrm{~mm} \mathrm{~s}^{-1}\right.$, rel. Intensität $\left.=100 \%\right)$. Nachdem die identische Mößbauer-Probe Atmosphärenluft ausgesetzt war ( $t=30 \mathrm{~min}$ ), konnten signifikante Änderungen ausgemacht werden. So zeigte der Hauptpeak mit einer Isomerieverschiebung von $\delta=0.39 \mathrm{~mm} \mathrm{~s}^{-1}$ und einer Quadrupolaufspaltung von $\Delta E_{\mathrm{Q}}=0.99 \mathrm{~mm} \mathrm{~s}^{-1}$ deutlich 
veränderte Mößbauer-Parameter. Zusätzlich konnte durch Oxidation die Bildung einer Eisen(II)-Spezies im high-spin-Zustand ausgemacht werden $\left(\delta=1.27 \mathrm{~mm} \mathrm{~s}^{-1}\right.$, $\Delta E_{\mathrm{Q}}=2.53 \mathrm{~mm} \mathrm{~s}^{-1}$, rel. Intensität $=15 \%$ ). Letztere legte die Vermutung nahe, dass die ursprüngliche, unter Inert-Bedingungen gehandhabte Eisen-Verbindung niedervalenten Ursprungs war.
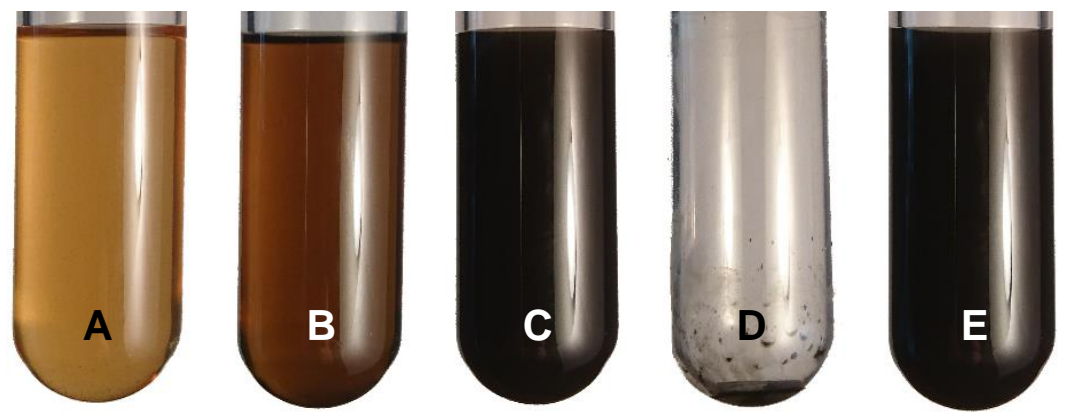

Abbildung 41. Makroskopische Betrachtung einer Reaktionslösung von $\mathrm{FeCl}_{2}$, TMEDA (4.0 Äq.) und MeMgCl (4.0 Äq.). Die ursprünglich gelbe $\mathrm{FeCl}_{2} / \mathrm{TMEDA}$-Lösung (A) wurde nach Zugabe von $\mathrm{MeMgCl}$ sofort dunkler (B). Nach $t=2$ min lag sie als schwarze Lösung vor (C). Ein schwarzer Feststoff verblieb (D), nachdem die Lösung (E) entfernt worden war.

In Anwesenheit von TMEDA war es erstmals möglich, Ethylferrate ( $\rightarrow$ Abbildung 42) und Butylferrate ( $\rightarrow$ Abbildung 43 ) kurzzeitig soweit $\mathrm{zu}$ stabilisieren, dass diese mittels ESI-Massenspektrometrie nachgewiesen werden konnten. So war $\mathrm{Et}_{4} \mathrm{Fe}(\mathrm{III})^{-}$das dominierende Anion nach Transmetallierung von $\mathrm{Fe}(\mathrm{acac})_{3}$ mit EtMgCl (4.0 Äq.) in Gegenwart von TMEDA (4.0 Äq.). Auch konnten hohe Signalintensitäten für $\mathrm{Et}_{3} \mathrm{Fe}(\mathrm{II})^{-}$beobachtet werden. Im Falle der Butylferrate war ein hohes Aufkommen von $\mathrm{Bu}_{4} \mathrm{Fe}(\mathrm{III})^{-}$erkennbar. Daneben waren in geringen Signalintensitäten die Eisen(II)-Spezies $\mathrm{Bu}_{3} \mathrm{Fe}^{-}, \mathrm{Bu}_{4} \mathrm{Fe}_{2} \mathrm{Cl}^{-}$sowie $\mathrm{Bu}_{5} \mathrm{Fe}_{2}^{-} \mathrm{zu}$ beobachten. Erstmals trat mit $\mathrm{Bu}_{2} \mathrm{Fe}^{-}$auch ein At-Komplex auf, der Eisen in der Oxidationsstufe $+I$ enthielt. Bei beiden Transmetallierungssystemen waren mit den Anionen der allgemeinen Form $\left[\mathrm{R}_{2}, \mathrm{Fe}, \mathrm{O}_{2}\right]^{-}(\mathrm{R}=\mathrm{Et}, \mathrm{Bu})$ erneut Spezies zu erkennen, die durch Reaktion mit Hintergrundsauerstoff entstanden waren.

Die Alkylferrate der höheren Homologen (Hex, Oct, Dec) zeigten bei Vorhandensein von TMEDA eine deutlich höhere zeitliche Stabilität, blieben sonst aber unbeeinflusst. Die ESI-Massenspektren nach Transmetallierung von $\mathrm{Fe}(\mathrm{acac})_{3}$ mit $\mathrm{BnMgCl}$ oder $\mathrm{Me}_{3} \mathrm{SiCH}_{2} \mathrm{MgCl}$ wiesen nicht länger zweikernige Aggregate auf. Die das Spektrum dominierenden, einkernigen Organoferrate dieser Systeme präsentierten sich hingegen unverändert. 


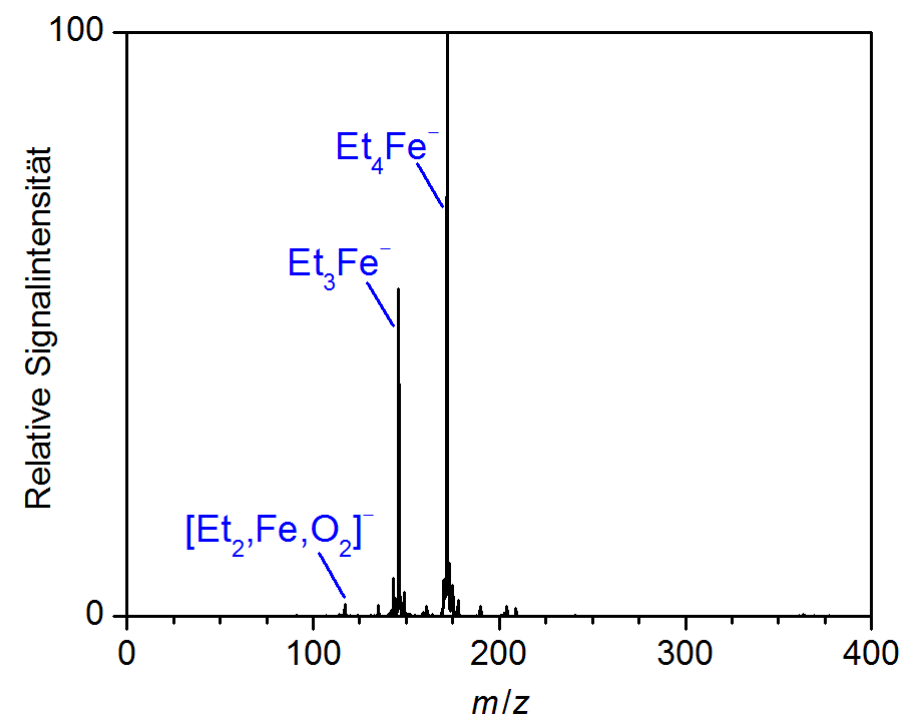

Abbildung 42. ESI-Massenspektrum (negativer lonenmodus) der Produkte aus der Reaktion von $\mathrm{Fe}(\mathrm{acac})_{3}$ (20 mM) mit TMEDA (4.0 Äq.) und EtMgCl (4.0 Äq.) in THF.

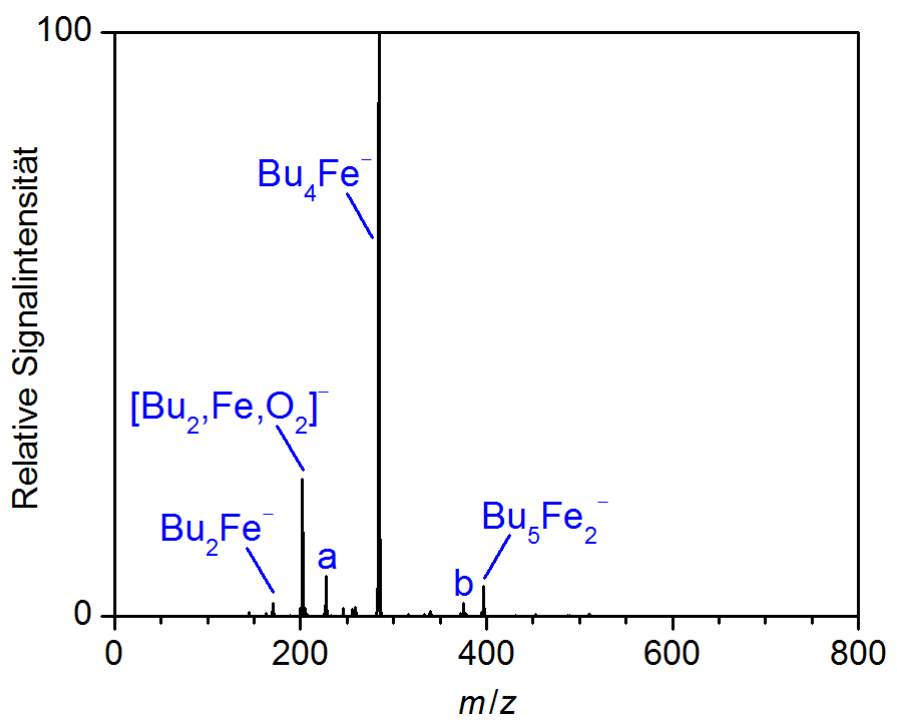

Abbildung 43. ESI-Massenspektrum (negativer lonenmodus) der Produkte aus der Reaktion von $\mathrm{Fe}(\mathrm{acac})_{3}$ (20 mM) mit TMEDA (4.0 Äq.) und BuMgCl (4.0 Äq.) in THF; $a=\mathrm{Bu}_{3} \mathrm{Fe}^{-}, \mathrm{b}=\mathrm{Bu}_{4} \mathrm{Fe}_{2} \mathrm{Cl}^{-}$. 


\subsubsection{Transmetallierung von $\mathrm{FeCl}_{2}(\mathrm{dppbz})_{2}$ mit $\mathrm{ArMgX}$}

In den letzten Jahren haben sich gängige Eisenvorläufer in Kombination mit bidentaten Phosphanliganden in Eisen-katalysierten Kreuzkupplungsreaktionen als besonders effektiv und effizient erwiesen (siehe Kapitel 1.2). Daher ist es von großem Interesse, die Auswirkung bidentater Phosphanliganden auf die Transmetallierung von Eisenvorläufern mit GrignardReagenzien im Detail zu untersuchen. Zumeist ist 1,2-Bis(diphenylphosphino)benzol (dppbz) der bidentate Phosphanligand der Wahl. Dieser ist mit $\mathrm{FeCl}_{2}(\mathrm{dppbz})_{2}$ direkt als Eisenpräkatalysator kommerziell zugänglich ( $\rightarrow$ Schema 9).<smiles>Cl[PH](c1ccccc1)(c1ccccc1)c1ccccc1P(c1ccccc1)(c1ccccc1)(c1ccccc1)P(Cl)(Cl)(CP(c1ccccc1)c1ccccc1)c1ccccc1</smiles>

Schema 9. Struktur von $\mathrm{FeCl}_{2}(\mathrm{dppbz}) 2$.

Die Transmetallierung von $\mathrm{FeCl}_{2}(\mathrm{dppbz})_{2}$ mit $\mathrm{PhMgCl}$ (4.0 Äq.) wurde anhand von ESI-Massenspektrometrie untersucht ( $\rightarrow$ Abbildung 44). Als hauptsächliches Produkt dieser Reaktion konnte die zweikernige Eisen(I)-Spezies $\mathrm{Ph}_{3} \mathrm{Fe}_{2}(\mathrm{ddpbz})^{-}$ausgemacht werden $\left(\rightarrow\right.$ Abbildung 45). Daneben war neben dem bereits bekannten Anion $\mathrm{Ph}_{3} \mathrm{Fe}(\mathrm{II})^{-}$eine ganze Reihe niedervalenter Ferrat-Komplexe zu beobachten ( $\rightarrow$ Tabelle 6$)$.

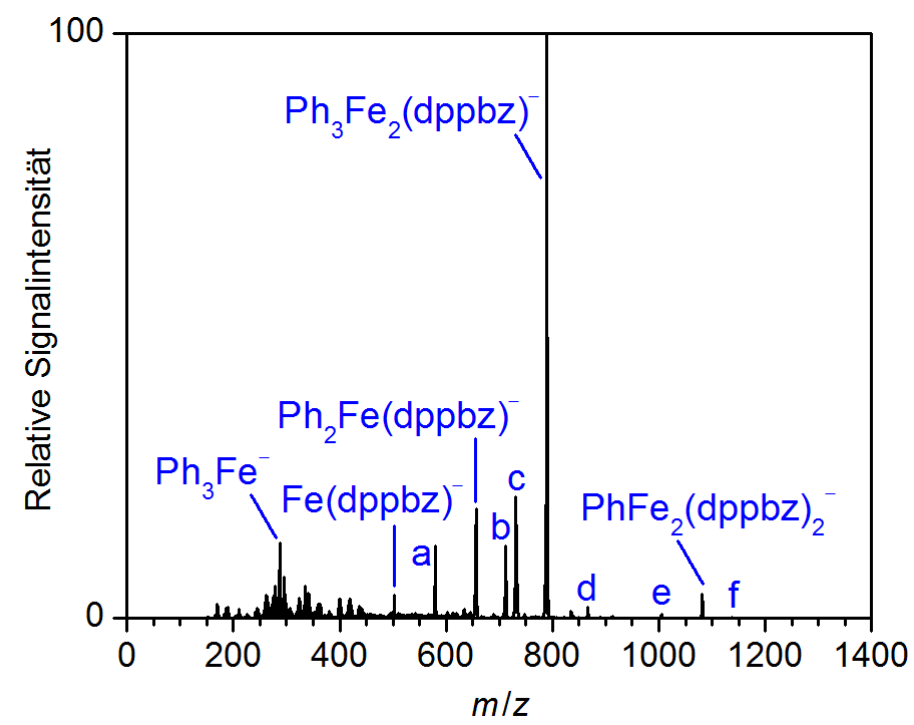

Abbildung 44. ESI-Massenspektrum (negativer lonenmodus) der Produkte aus der Reaktion von $\mathrm{FeCl}_{2}\left(\mathrm{dppbz}_{2}\right.$ $(10 \mathrm{~mm})$ mit $\mathrm{PhMgCl}$ (4.0 Äq.) in THF; $\mathrm{a}=\mathrm{PhFe}(\mathrm{dppbz})^{-}, \quad \mathrm{b}=\mathrm{Ph}_{2} \mathrm{Fe}_{2}(\mathrm{dppbz})^{-}, \quad \mathrm{c}=\left[\mathrm{Ph}_{3} \mathrm{Fe}(\mathrm{dppbz})-\mathrm{H}\right]^{-}+$ $\mathrm{Ph}_{3} \mathrm{Fe}(\mathrm{dppbz})^{-} \mathrm{d}=\mathrm{Ph}_{4} \mathrm{Fe}_{2}(\mathrm{dppbz})^{-}, \mathrm{e}=\mathrm{Fe}_{2} \mathrm{H}(\mathrm{dppbz})_{2}^{-}, \mathrm{f}=\mathrm{PhFe} \mathrm{H}_{3} \mathrm{H}(\mathrm{dppbz})_{2}{ }^{-}$. 


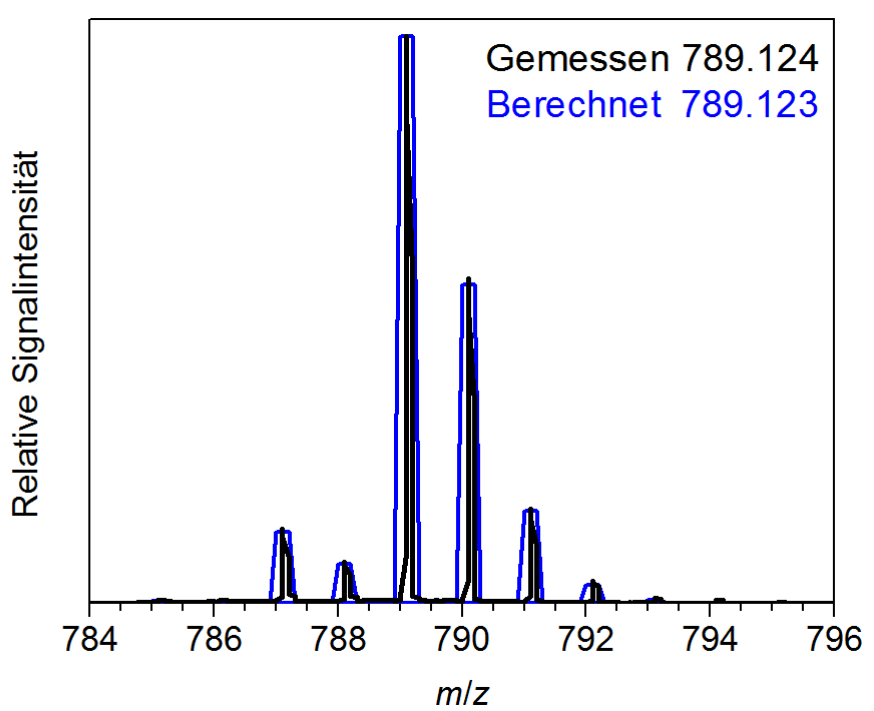

Abbildung 45. Vergleich des gemessenen (schwarz) und des simulierten (blau) Isotopenmusters von $\mathrm{Ph}_{3} \mathrm{Fe}_{2}(\mathrm{dppbz})^{-}$.

In einem Vergleichsexperiment konnten über Transmetallierung von $\mathrm{FeCl}_{2}$ mit $\mathrm{PhMgCl}$ (4.0 Äq.) in Anwesenheit von dppbz (2.0 Äq.) die analogen Spezies erzeugt werden $\left(\rightarrow\right.$ Tabelle 6). Das entsprechende Experiment mit ${ }^{57} \mathrm{FeCl}_{2}$ lieferte ein Mößbauer-Spektrum, was auf ein Vorliegen vieler sich überlappender Spezies mit wenig charakteristischen Mößbauer-Parametern hindeutete ( $\rightarrow$ Abbildung 46). Das Vorliegen niedervalenter EisenSpezies konnte mit diesem Experiment abschließend weder bestätigt noch ausgeschlossen werden.

Tabelle 6. Übersicht über die Organoferrate, entstanden durch Transmetallierung von $\mathrm{FeCl}_{2}(\mathrm{dppbz})_{2}(10 \mathrm{mM}) \mathrm{mit}$ $\mathrm{PhMgCl}$ (4.0 Äq.) in THF inklusive ihrer durchschnittlichen Oxidationszahlen.

\begin{tabular}{lc}
\hline \multicolumn{1}{c}{ Spezies } & $\begin{array}{c}\text { Durchschnittliche } \\
\text { Oxidationszahlen }\end{array}$ \\
\hline $\mathrm{Ph}_{3} \mathrm{Fe}^{-}$ & 2.0 \\
$\mathrm{Fe}(\mathrm{dppbz})^{-}$ & -1.0 \\
$\mathrm{PhFe}(\mathrm{dppbz})^{-}$ & 0 \\
$\mathrm{Ph}_{2} \mathrm{Fe}(\mathrm{dppbz})^{-}$ & 1.0 \\
$\mathrm{Ph}_{2} \mathrm{Fe}(\mathrm{dppbz})^{-}$ & 0.5 \\
$\mathrm{Ph}_{3} \mathrm{Fe}(\mathrm{dppbz})^{-}$ & 2.0 \\
$\mathrm{Ph}_{3} \mathrm{Fe}(\mathrm{dppbz})^{-}$ & 1.0 \\
$\mathrm{Ph}_{4} \mathrm{Fe}_{2}(\mathrm{dppbz})^{-}$ & 1.5 \\
$\mathrm{Fe}_{2} \mathrm{H}(\mathrm{dppbz})_{2}^{-}$ & 0 \\
$\mathrm{PhFeH}^{-}$ & $1 \mathrm{dppbz})^{-}$ \\
$\mathrm{PhFe}_{3} \mathrm{H}(\mathrm{dppbz})_{2}{ }^{-}$ & 1.0 \\
\hline
\end{tabular}




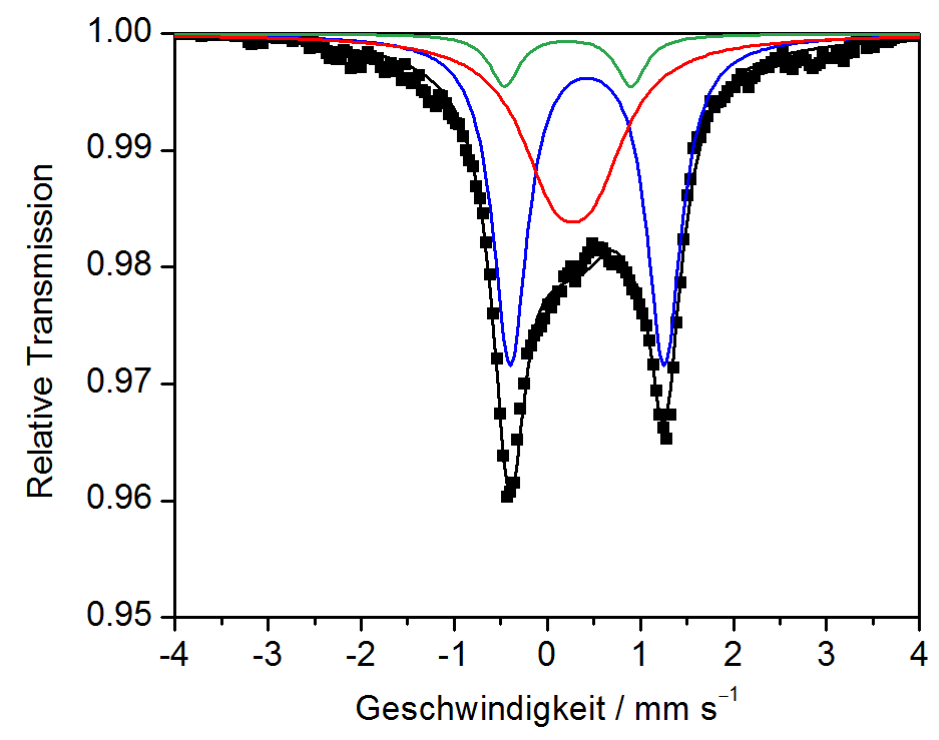

Abbildung 46. Mößbauer-Spektrum einer gefrorenen Lösung der Produkte aus der Reaktion von ${ }^{57} \mathrm{FeCl}_{2} \mathrm{mit}_{\mathrm{dppbz}}$ (2.0 Äq.) und PhMgCl (4.0 Äq.) in THF ( $T=80 \mathrm{~K}) ; \delta$ (blau) $=0.43 \mathrm{~mm} \mathrm{~s}^{-1}, \Delta E_{\mathrm{Q}}$ (blau) $=1.65 \mathrm{~mm} \mathrm{~s}^{-1}$, rel. Intensität $($ blau $)=55 \% ; \delta($ rot $)=0.28 \mathrm{~mm} \mathrm{~s}^{-1}, \Delta E_{\mathrm{Q}}($ rot $)=0.42 \mathrm{~mm} \mathrm{~s}^{-1}$, rel. Intensität $($ rot $)=38 \% ; \delta($ grün $)=0.22 \mathrm{~mm} \mathrm{~s}^{-1}$, $\Delta E_{\mathrm{Q}}$ (grün) $=1.36 \mathrm{~mm} \mathrm{~s}^{-1}$, rel. Intensität (grün) $=7 \%$.

Wurde $\mathrm{FeCl}_{2}(\mathrm{dppbz})_{2}$ mit $\mathrm{MesMgBr}$ (4.0 Äq.) transmetalliert, führte dies erneut zur primären Bildung von $\mathrm{Mes}_{3} \mathrm{Fe}(\mathrm{II})^{-}$. Im Gegensatz dazu führte die Umsetzung von $\mathrm{FeCl}_{2}(\mathrm{dppbz})_{2}$ mit $\left(\mathrm{Ar}_{\mathrm{F}}\right) \mathrm{MgBr}$ zur Neubildung der Eisen(II)-Spezies $\left(\mathrm{Ar}_{\mathrm{F}}\right)_{3} \mathrm{Fe}(\mathrm{dppbz})^{-}$, die neben den bereits bekannten Anionen $\left(\mathrm{Ar}_{\mathrm{F}}\right)_{3} \mathrm{Fe}(\mathrm{II})^{-}$und $\left(\mathrm{Ar}_{\mathrm{F}}\right)_{4} \mathrm{Fe}(\mathrm{III})^{-}$in hoher Signalintensität vorlag. 


\subsubsection{Transmetallierung von $\mathrm{FeCl}_{2}(\mathrm{dppbz})_{2}$ mit AlkMgCl}

Wurde $\mathrm{FeCl}_{2}(\mathrm{dppbz})_{2}$ mit $\mathrm{MeMgCl}$ oder den höheren Homologen der alkylischen GrignardReagenzien (Bu, Hex, Oct, Dec) umgesetzt, konnten in den ESI-Massenspektren im negativen Ionenmodus ausschließlich rein anorganische Mg-Spezies beobachtet werden. Einzige Ausnahme stellte die Transmetallierung mit $\mathrm{EtMgCl}$ dar, die zur Ausbildung niedervalenter Ferrat-Komplexe führte ( $\rightarrow$ Abbildung 47). Die Hauptspezies bildete dabei das Anion $\left[\mathrm{Et}_{2} \mathrm{Fe}(\mathrm{dppbz})-2 \mathrm{H}\right]^{-}$. Daneben waren mit $\mathrm{Fe}(\mathrm{dppbz})^{-}$sowie $\mathrm{EtFe}_{2}(\mathrm{dppbz})^{-}$und $\mathrm{FeH}(\mathrm{dppbz})^{-}$ Organoferrate mit Eisenzentren in den Oxidationsstufen $-\mathrm{I}$ und 0 detektierbar. Besonders auffällig war, dass im Falle der Transmetallierung von $\mathrm{FeCl}_{2}(\mathrm{dppbz})_{2}$ mit $\mathrm{MeMgCl}$ erstmals auch eisenhaltige Kationen detektiert werden konnten. So zeigten sich im hohen Massenbereich die Kationen $\mathrm{Fe}(\mathrm{dppbz})_{2}{ }^{+}$und $\mathrm{MeFe}(\mathrm{dppbz})_{2}{ }^{+}$in niedriger relativer Signalintensität. Durch die Reaktion von $\mathrm{FeCl}_{2}(\mathrm{dppbz})_{2}$ mit $\mathrm{BnMgCl}$ oder $\mathrm{Me}_{3} \mathrm{SiCH}_{2} \mathrm{MgCl}$ konnten einzig Organoferrate gebildet werden, die bereits in Abwesenheit von Additiven und Liganden in Lösung vorlagen (siehe Kapitel 4.1.1.6 und 4.1.1.7).

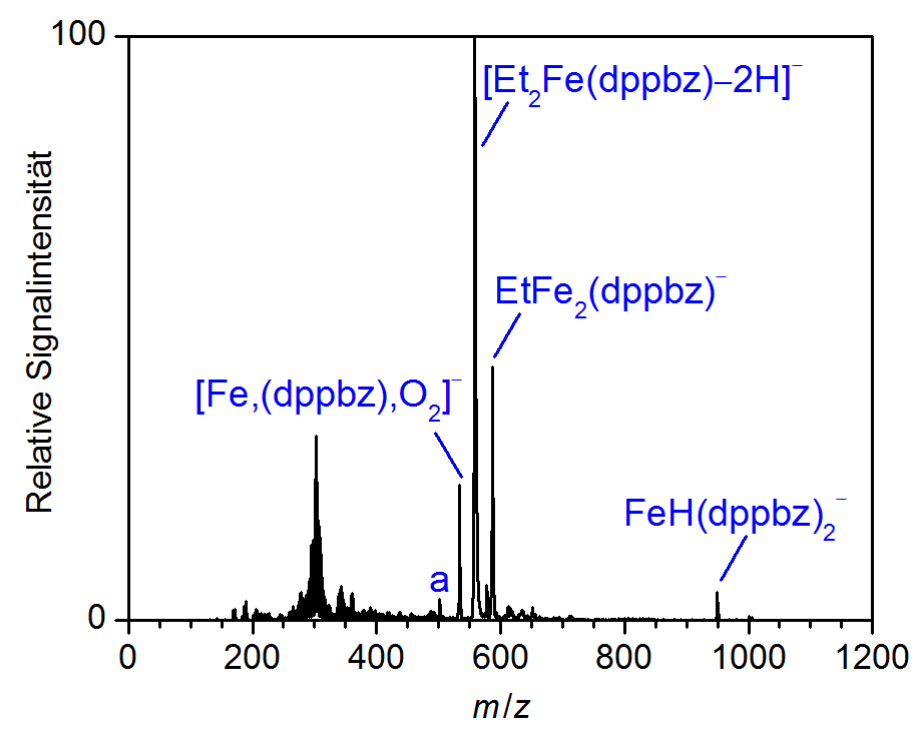

Abbildung 47. ESI-Massenspektrum (negativer lonenmodus) der Produkte aus der Reaktion von $\mathrm{FeCl}_{2}(\mathrm{dppbz})_{2}$ (10 mM) mit EtMgCl (4.0 Äq.) in THF; $a=F e(d p p b z)^{-}$. 


\subsubsection{Transmetallierung von $\mathrm{Fe}(\mathrm{acac})_{3}$ mit $\mathrm{PhMgCl}$ oder $\mathrm{MeMgCl}$ in Anwesenheit weiterer Additive und Liganden}

Neben TMEDA und dppbz wurden weitere Additive und Liganden hinsichtlich ihrer Wirkung auf die Transmetallierung von $\mathrm{Fe}(\mathrm{acac})_{3}$ mit $\mathrm{PhMgCl}$ oder $\mathrm{MeMgCl}$ untersucht $(\rightarrow$ Schema 10). Zusätzlich wurde die Auswirkung von $\mathrm{LiCl}$ als rein anorganisches Additiv näher beleuchtet.<smiles>CN1CCCC1=O</smiles>

NMP<smiles>c1ccc(P(c2ccccc2)P(c2ccccc2)c2ccccc2)cc1</smiles>

$\mathrm{PPh}_{3}$

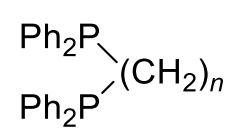

dppe $(n=2)$ $\operatorname{dppp}(n=3)$

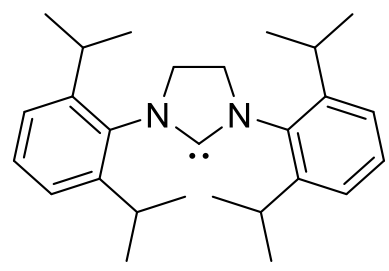

SIPr

Schema 10. Übersicht über die organischen Additive und Liganden, deren Effekt auf die Transmetallierung von $\mathrm{Fe}(\mathrm{acac})_{3}$ durch $\mathrm{PhMgCl}$ oder $\mathrm{MeMgCl}$ mittels ESI-Massenspektrometrie untersucht wurde.

Unabhängig vom Transmetallierungssystem hatte der Zusatz von NMP Effekte auf das ESI-Massenspektrum im negativen lonenmodus, die schon in Gegenwart von TMEDA beobachtet werden konnten. So zeigten einkernige Organoferrate eine höhere zeitliche Stabilität bei gleichzeitigem Verschwinden der höheren Aggregate. Identische Auswirkungen hatte die Präsenz von $\mathrm{LiCl}$, SIPr, dppp und dppe auf eine Probenlösung von $\mathrm{Fe}(\mathrm{acac})_{3} / 4 \mathrm{PhMgCl}$, wobei diese Additive keine sichtbaren Auswirkungen auf die Bildung der Methylferrate hatten. Einzige Ausnahme war der bidentate Phosphanligand dppe, dessen Gegenwart mit $\mathrm{Fe}(\mathrm{dppe}){ }_{2}{ }^{+}$und $\mathrm{MeFe}(\mathrm{dppe})_{2}{ }^{+}$erneut kationische Eisen-Spezies hervorbrachte. $\mathrm{Zu}$ einem interessanten Ergebnis führte der Zusatz von $\mathrm{PPh}_{3}$. So konnten mit $\mathrm{Ph}_{2} \mathrm{Fe}\left(\mathrm{PPh}_{3}\right)^{-}$ und $\mathrm{Ph}_{3} \mathrm{Fe}_{2}\left(\mathrm{PPh}_{3}\right)^{-}$Eisen(I)-haltige Spezies soweit stabilisiert werden, dass deren Detektion mittels ESI-Massenspektrometrie möglich war. Zusätzlich erschien mit $\mathrm{Ph}_{8} \mathrm{Fe}_{5}{ }^{-}$erstmals auch ein fünfkerniges Aggregat. Im Gegensatz dazu hatte $\mathrm{PPh}_{3}$ als Additiv keinerlei Auswirkungen auf die Bildung und Stabilität von Methylferraten. 


\subsubsection{Transmetallierung von $\mathrm{FeCl}_{2}$ mit $\mathrm{R}_{2} \mathrm{Zn}$ in Anwesenheit bidentater Phosphanliganden}

In Abwesenheit von Additiven und Liganden waren durch Transmetallierung von $\mathrm{FeCl}_{2}$ mit $\mathrm{R}_{2} \mathrm{Zn}(\mathrm{R}=\mathrm{Ph}, \mathrm{Et})$ keine anionischen oder kationischen Eisenkomplexe zugänglich (siehe Kapitel 4.1.1.10). Hingegen bewirkte der Zusatz bidentater Phosphanliganden die Entstehung kationischer Eisen(I)-Spezies des Typs $\mathrm{FeL}_{2}{ }^{+}(\mathrm{L}=\mathrm{dppe}$, dppp, dppbz), wobei nur in Anwesenheit von dppbz zusätzlich Kationen der allgemeinen Form $\mathrm{RFe}(\mathrm{dppbz})_{2}{ }^{+}(\mathrm{R}=\mathrm{Ph}, \mathrm{Et})$ mit Eisen(II)-Zentrum in sehr hoher Signalintensität detektiert werden konnten $(\rightarrow$ Abbildung 48).

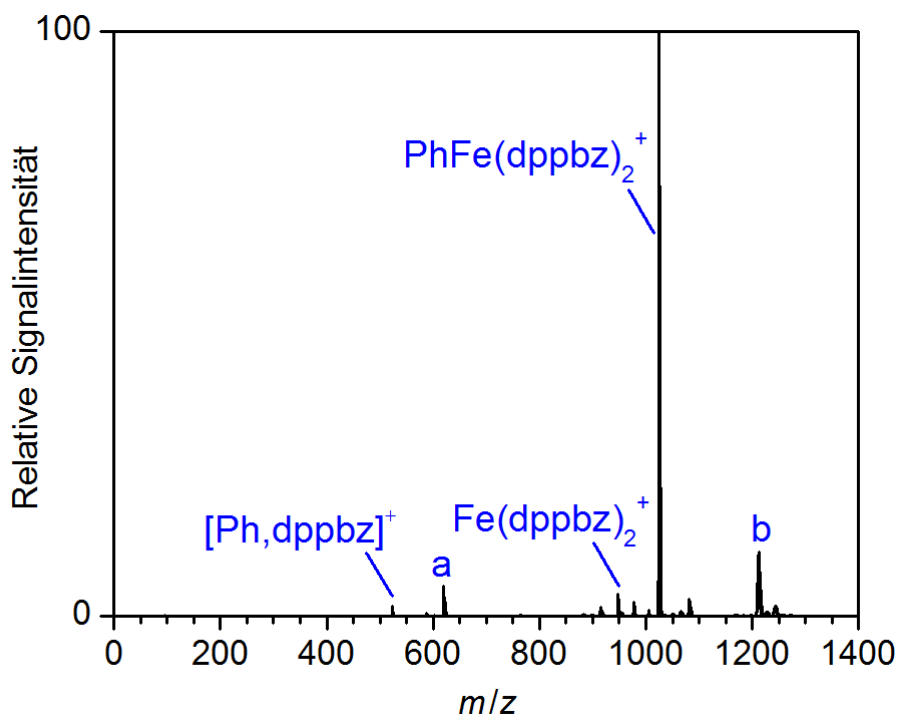

Abbildung 48. ESI-Massenspektrum (positiver lonenmodus) der Produkte aus der Reaktion von $\mathrm{FeCl}_{2}(\mathrm{dppbz})_{2}$ (10 mM) und $\mathrm{Ph}_{2} \mathrm{Zn}$ (4.0 Äq.) in THF; $\mathrm{a}=\left[\mathrm{Ph}, \mathrm{Zn},(\mathrm{dppbz}), \mathrm{O}_{2}\right]^{+}, \mathrm{b}=\mathrm{Ph}_{2} \mathrm{Zn}_{2} \mathrm{Cl}(\mathrm{dppbz}) 2^{+}$.

Für $\mathrm{R}=\mathrm{Et}$ war mit $\mathrm{FeH}(\mathrm{dppbz})_{2}{ }^{+}$ein Kation zugänglich, das als Produkt einer $\beta$-H-Eliminierung angesehen werden kann ( $\rightarrow$ Schema 11). Die Signalintensität dieser Spezies nahm im weiteren Verlauf der Messung stark zu und belegte damit erneut die geringe zeitliche Stabilität geladener Organoeisen-Spezies, die organische Reste mit $\beta$-H-Atomen tragen.

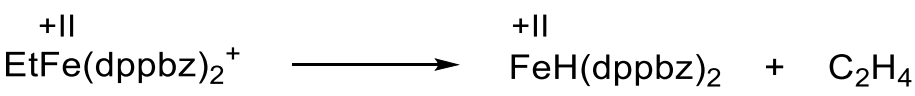

Schema 11. Bildung der Spezies $\mathrm{FeH}(\mathrm{dppbz}) 2^{+}$über $\beta-\mathrm{H}$-Eliminierung. 


\subsubsection{Einfluss von Additiven und Liganden auf die Transmetallierung}

Durch Transmetallierung von $\mathrm{Fe}(\mathrm{acac})_{3}$ mit Grignard-Reagenzien in Abwesenheit oder Anwesenheit verschiedener Additive konnte eine Vielzahl ligandfreier Organoferrate gebildet werden ( $\rightarrow$ Tabelle 7 ). Auffällig ist die zumeist hohe Dominanz der Organoferrate in den Oxidationsstufen + II und +III.

Tabelle 7. Übersicht über Organoferrate, entstanden aus der Reaktion von Fe(acac) 3 mit verschiedenen GrignardReagenzien (4.0 Äq.) in Abwesenheit und Anwesenheit von TMEDA inklusive ihrer Oxidationsstufen. In rot markiert sind die Organoferrate, die in Anwesenheit von TMEDA nicht länger beobachtet werden konnten.

\begin{tabular}{|c|c|c|c|c|c|c|c|c|c|c|c|c|}
\hline Spezies & $\begin{array}{l}\text { Oxidations } \\
\text {-stufe }\end{array}$ & $\mathbf{R}=\mathbf{P h}$ & Mes & $\operatorname{Ar}_{\mathrm{F}}{ }^{a}$ & Me & $\mathrm{Et}^{b}$ & $\mathrm{Bu}^{b}$ & Hex & Oct & Dec & $\mathrm{Bn}$ & $\mathrm{Me}_{3} \mathrm{SiCH}_{2}$ \\
\hline $\mathrm{R}_{2} \mathrm{Fe}^{-}$ & 1.0 & $-^{c}$ & - & - & - & - & $+^{d}$ & - & - & - & - & - \\
\hline $\mathrm{R}_{12} \mathrm{Fe}_{8^{-}}$ & 1.4 & - & - & - & $++^{e}$ & - & - & - & - & - & - & - \\
\hline $\mathrm{R}_{7} \mathrm{Fe}_{4}^{-}$ & 1.5 & + & - & - & - & - & - & - & - & - & - & - \\
\hline $\mathrm{R}_{13} \mathrm{Fe}_{8^{-}}$ & 1.5 & - & - & - & + & - & - & - & - & - & - & - \\
\hline $\mathrm{R}_{3} \mathrm{Fe}^{-}$ & 2.0 & + & ++ & ++ & + & + & + & + & + & + & + & + \\
\hline $\mathrm{R}_{5} \mathrm{Fe}_{2}^{-}$ & 2.0 & - & - & - & - & - & + & + & - & - & + & - \\
\hline $\mathrm{R}_{6} \mathrm{Fe}_{2^{-}}$ & 2.5 & - & - & - & - & - & - & - & - & - & + & - \\
\hline $\mathrm{R}_{4} \mathrm{Fe}^{-}$ & 3.0 & + & - & + & + & + & + & ++ & ++ & ++ & + & ++ \\
\hline
\end{tabular}

a $\mathrm{Ar}_{\mathrm{F}}=3,5-\left(\mathrm{CF}_{3}\right)_{2}-\left(\mathrm{C}_{6} \mathrm{H}_{3}\right)$. ${ }^{b}$ Nur in Anwesenheit von TMEDA detektierbar. ${ }^{c}$ Spezies wurde nicht beobachtet (Signalintensität $\leq 2 \%$ in Relation zum Hauptpeak). ${ }^{d}$ Spezies wurde in niedriger oder mittlerer Signalintensität beobachtet (2-50\%). ${ }^{e}$ Spezies wurde in hoher Signalintensität beobachtet $(\geq 50 \%)$.

Wie zuvor ausführlich diskutiert (siehe Kapitel 4.1.1.11), kann die Bildung Eisen(III)-haltiger Anionen unter reduktiven Bedingungen über eine Redoxdisproportionierung von $\mathrm{R}_{3} \mathrm{Fe}(\mathrm{II})^{-}$ erklärt werden. Gleichzeitig müssen dabei niedervalente Eisen(I)-Spezies $\mathrm{R}_{2} \mathrm{Fe}^{-}$entstehen. In Anwesenheit von TMEDA war es erstmals möglich, mit $\mathrm{Bu}_{2} \mathrm{Fe}(\mathrm{I})^{-}$solch eine Spezies mittels ESI-Massenspektrometrie nachzuweisen. Dies untermauert das mögliche Auftreten einer Redoxdisproportionierung. Dass keine weiteren dieser elektronenreichen und hochsensitiven Eisenverbindungen nachgewiesen werden konnten, ist durch eine sehr schnell ablaufende Überreduktion dieser Komplexe zu erklären, die zur Bildung eines neutralen Feststoffes führte. Dieser enthielt etwa $40 \%$ des ursprünglich eingesetzten Eisens, wohingegen die restlichen $60 \%$ als Eisen(III)-Spezies in der abgetrennten Lösung verblieben. Aus einer Bilanzierung der Elektronen lässt sich abschätzen, dass der Feststoff Eisen in einer durchschnittlichen Oxidationsstufe von 0.5 enthält. Der elektronenreiche Charakter von Eisen im Feststoff ist Grund für dessen hohe Oxidationsempfindlichkeit an Atmosphärenluft. 
TMEDA ist eines der gängigsten Additive in Eisen-katalysierten Kumada-Kreuzkupplungsreaktionen und ist bekannt dafür, die Effizienz dieser Reaktionen zu erhöhen. ${ }^{[19 a]}$ Da die Anwesenheit von TMEDA stets zur Dominanz einkerniger Organoferrate führte, scheinen polynukleare Eisen-organische Spezies als mögliche Katalysatoren dieser Reaktion keine Rolle zu spielen. Betrachtet man die Werte der elektrischen Leitfähigkeiten entsprechender Reaktionslösungen, so fällt auf, dass diese in Gegenwart von TMEDA deutlich niedriger ausfallen ( $\rightarrow$ Tabelle 8). Daraus lässt sich ablesen, dass das Fernbleiben polynuklearer Aggregate nicht mit einer Mehrbildung ionischer Spezies einhergeht.

Tabelle 8. Spezifische, elektrische Leitfähigkeit $\kappa / \mu \mathrm{S} \mathrm{cm}^{-1}$ der Organoferrate, entstanden durch Transmetallierung von $\mathrm{Fe}(\mathrm{acac})_{3}(50 \mathrm{mM})$ mit Grignard-Reagenzien in Anwesenheit von TMEDA in THF bei $T=273 \mathrm{~K}$.

\begin{tabular}{cc}
\hline Probenlösung in THF & $\boldsymbol{\kappa} / \boldsymbol{\mu} \mathbf{~ c m}^{-1}$ \\
\hline $\mathrm{Fe}(\mathrm{acac})_{3}+4$ TMEDA + 4 PhMgCl & $83 \pm 5$ \\
$\mathrm{Fe}(\mathrm{acac})_{3}+4 \mathrm{TMEDA}+4 \mathrm{MeMgCl}$ & 0 \\
$\mathrm{Fe}(\mathrm{acac})_{3}+4 \mathrm{TMEDA}+4 \mathrm{BnMgCl}$ & $20 \pm 4$ \\
\hline
\end{tabular}

Als weiterer Effekt von TMEDA sticht dessen stabilisierende Wirkung auf einkernige Organoferrate heraus. Wie ESI-massenspektrometrische Untersuchungen zeigten, ist dies nicht auf eine direkte Bindung von TMEDA an Eisen zurückzuführen. Vielmehr erscheint TMEDA nur in Mg-haltigen Kationen. Bestätigt wird diese Tatsache durch Arbeiten von Bedford und Mitarbeitern, die in der Vergangenheit nachweisen konnten, dass TMEDA im Reaktionsverlauf Eisen-katalysierter Kreuzkupplungsreaktionen nicht an relevante Eisenintermediate bindet. ${ }^{[82]}$ So muss der stabilisierende Einfluss von TMEDA indirekten Ursprungs sein. Denkbar ist, dass TMEDA an neutrale Organoeisen-Komplexe bindet, die nicht direkt am Katalysezyklus beteiligt sind, aber über chemische Gleichgewichte die AtKomplexe in Lösung beeinflussen. Allerdings ließen sich mit Mößbauer-Spektroskopie keine Hinweise darauf finden, dass solche Neutralteilchen tatsächlich gebildet werden. So scheinen die Organoferrate durch Wechselwirkung mit den Mg-TMEDA-Kationen stabilisiert zu werden. Die Wirkung von NMP als weitere $N$-Donorbase mit effizienzsteigender Wirkung auf Eisenkatalysierte Kreuzkupplungsreaktionen ist vergleichbar mit der von TMEDA und ist auf einen ähnlichen Ursprung zurückzuführen. ${ }^{[17 c, 39 i]}$

Um Eisen-katalysierte Kreuzkupplungsreaktionen effizienter zu gestalten, können alternativ zu TMEDA oder NMP Phosphanliganden eingesetzt werden. ${ }^{[19 e]}$ Im Rahmen dieser Arbeit konnte gezeigt werden, dass dppbz als bidentater Ligand speziell niedervalente Organoferrate mit 
Eisen in den Oxidationsstufen zwischen $-\mathrm{I}$ und $+\mathrm{I}$ stabilisiert. Primär wirken Phosphanliganden in Metallkomplexen als $\sigma$-Donoren ( $\rightarrow$ Schema 12). ${ }^{[83]}$ Daneben besitzen sie die Fähigkeit zur $\pi$-Rückbindung. Diese ist entscheidend, um die Elektronendichte an elektronenreichen Metallen wie Eisen(I) zu reduzieren, indem diese in das $\sigma^{*}$-Orbital der Phosphor-KohlenstoffBindung abgegeben wird. Daneben wirkt die koordinative Absättigung des bidentaten Phosphanliganden durch die Bindung an die niedervalente Eisen-Spezies zusätzlich stabilisierend.

(a)

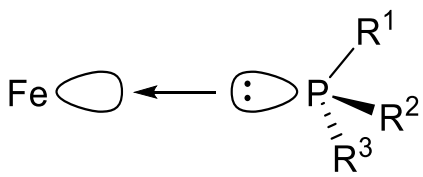

(b)

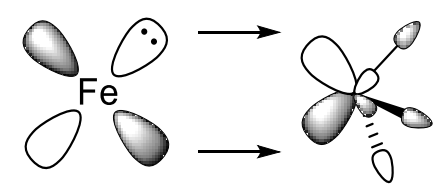

Schema 12. Darstellung der Bindungsinteraktionen zwischen Eisenzentrum und Phosphanligand mit den beteiligten Orbitalen: (a) $\sigma$-Donor-Bindung; (b) $\pi$-Rückbindung. ${ }^{\text {[83a] }}$

Die Stärke der $\pi$-Rückbindung ist abhängig von den elektronischen Eigenschaften der Phosphorsubstituenten. ${ }^{[83 b]}$ Phosphanliganden mit elektronegativen Substituenten sind aufgrund des niedrigeren Energieniveaus des $\sigma^{*}$-Orbitals der Phosphor-Kohlenstoff-Bindung die besseren $\pi$-Rückbinder. Somit hat dppbz mit einem Rückgrat aus $\mathrm{sp}^{2}$-hybridisierten Kohlenstoffatomen einen stärkeren $\pi$-Rückbindungscharakter in Relation zu dppe und dppp, deren Phosphoratome mit weniger elektronegativen $\mathrm{sp}^{3}$-Kohlenstoffatomen verbrückt sind. Als Folge der elektronisch günstigen Eigenschaften ist es nur mit dppbz möglich, elektronenreiche, niedervalente Organoferrate soweit zu stabilisieren, dass ihre Detektion mittels ESI-Massenspektrometrie als Anionen möglich war. Ähnliche elektronische Eigenschaften wie dppbz besitzt $\mathrm{PPh}_{3}$. Auch mit diesem Liganden ließen sich elektronenreiche Organoferrate mit Eisen in der Oxidationsstufe $+\mathrm{I}$ gut nachweisen.

In seltenen Fällen können Phosphan-haltige Eisen-Komplexe auch als Kationen vorliegen, speziell dann, wenn zwei Äquivalente dieser Liganden an Eisen gebunden sind. Besonders deutlich tritt dies zutage, wenn Organozink-Reagenzien zur Transmetallierung eingesetzt werden. Die Kombination der geringeren Reaktivität der Organozink-Reagenzien mit der koordinativen Absättigung durch Anlagerung zweier Liganden und das daraus entstehende, vergleichsweise elektronenarme Eisenzentrum sorgen dafür, dass in diesen Fällen die eisenhaltigen Kationen sogar dominieren. 
Bei der Transmetallierung von $\mathrm{FeCl}_{2}(\mathrm{dppbz})_{2}$ mit $\mathrm{EtMgCl}$ entsteht eine Spezies der allgemeinen Form $\left[\mathrm{Et}_{2} \mathrm{Fe}(\mathrm{dppbz})-2 \mathrm{H}\right]^{-}$. Denkbar ist, dass hier eine Eisen(I)-Spezies vorliegt, die neben dem intakten Phosphanliganden zwei $\mathrm{C}_{2} \mathrm{H}_{4}$-Einheiten trägt ( $\rightarrow$ Schema 13). Allgemein kann die Bindungsweise von Alken-Einheiten an Metallzentren auf zwei Arten beschrieben werden. ${ }^{[84]}$ Zum einen kann das Metall koordinativ an das $\pi$-System des Alkens binden. Zum anderen ist die Ausbildung eines Metalla-Cyclopropan-Systems denkbar. Die reale Bindungsweise eines Alkens an ein Metall, üblicherweise beschrieben durch das DewarChatt-Duncanson-Modell[ ${ }^{85]}$, liegt zumeist zwischen diesen beiden Extrema. Generell gilt, dass Metalle in niedrigen Oxidationsstufen wie Eisen(I) gute $\pi$-Donoren sind und Cyclopropan-artige Substituenten bevorzugen. So ist diese Bindungsweise für $\left[\mathrm{Et}_{2} \mathrm{Fe}(\mathrm{dppbz})-2 \mathrm{H}\right]^{-}$ wahrscheinlicher, auch wenn diese bei Vorhandensein zweier $\mathrm{C}_{2} \mathrm{H}_{4}$-Einheiten koordinativ größeren Einschränkungen gegenübersteht. Auch wäre eine Dimerisierung der beiden EthenEinheiten denkbar, sodass sich eine Metallacyclopenten-Struktur entwickelt.<smiles>CCC(CC)P(c1ccccc1)P(c1ccccc1)c1ccccc1</smiles>

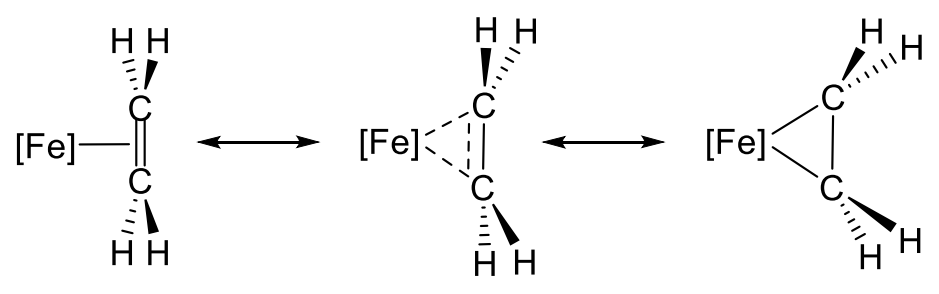

Schema 13. Strukturvorschlag für $\left[\mathrm{Et}_{2} \mathrm{Fe}(\mathrm{dppbz})-2 \mathrm{H}\right]^{-}$. Die Bindungsweise der $\mathrm{C}_{2} \mathrm{H}_{4}$-Einheiten liegt zwischen Metall-Alken-Addukt (links) und Metallacyclopropan (rechts) vor. ${ }^{[84,85]}$

Für $N$-heterocyclische Carbene ist bekannt, dass diese an Eisen binden und stabile Strukturen ausbilden können. ${ }^{[366,86]}$ Solche Spezies konnten mittels ESI-Massenspektrometrie im Rahmen dieser Arbeit nicht nachgewiesen werden. Durch den stark elektronendonierenden Effekt des NHC-Liganden auf das Eisenzentrum scheinen diese Spezies auf Stufe der Neutralkomplexe stehenzubleiben. Die Übertragung eines weiteren organischen Substituenten auf diese bereits sehr elektronenreichen Komplexe scheint elektronisch ungünstig. 


\subsection{Reaktivität gegenüber organischen (Pseudo)-Halogeniden}

Die in situ erzeugten Organoferrate sollen in ihrer Reaktivität gegenüber organischen Substraten charakterisiert werden. Dabei soll der Vergleich ihrer Reaktivität in Lösung mit der spezifischen, bimolekularen Gasphasenreaktivität definierter Ferrat-Komplexe für ein breiteres mechanistisches Verständnis Eisen-katalysierter Kreuzkupplungsreaktionen auf Stufe der Interaktion mit organischen Substraten sorgen.

\subsubsection{Reaktivität in Lösung}

Als denkbar einfachstes Experiment zur Analyse der Reaktivität von Organoferraten in Lösung können mögliche organische Reaktionspartner in definierter Menge direkt in die entsprechenden Reaktionslösungen gegeben werden, bevor diese untersucht werden.

\subsubsection{Reaktivität von Phenylferraten gegenüber R'X}

Zu Reaktionslösungen von Ferrat-Komplexen, die durch Transmetallierung von $\mathrm{Fe}(\mathrm{acac})_{3}$ mit $\mathrm{PhMgCl}$ (4.0 Äq.) gebildet werden konnten, wurden Alkylhalogenide R'X (0.5 Äq.; R' = Et, 'Pr, $\mathrm{Bu} ; \mathrm{X}=\mathrm{Cl}, \mathrm{Br}$, I) gegeben. 'Dabei konnte in allen Fällen in Anwesenheit und Abwesenheit von TMEDA die Bildung der heteroleptischen Eisen(III)-Spezies $\mathrm{Ph}_{3} \mathrm{FeR}^{\prime-}$ als Produkt der Addition des Substrates an einen Organoeisen-Komplex beobachtet werden. ( $\rightarrow$ Abbildung 49). Mit Mößbauer-Spektroskopie gelang es nicht, diese kurzlebigen Eisen(III)-Spezies zu detektieren.

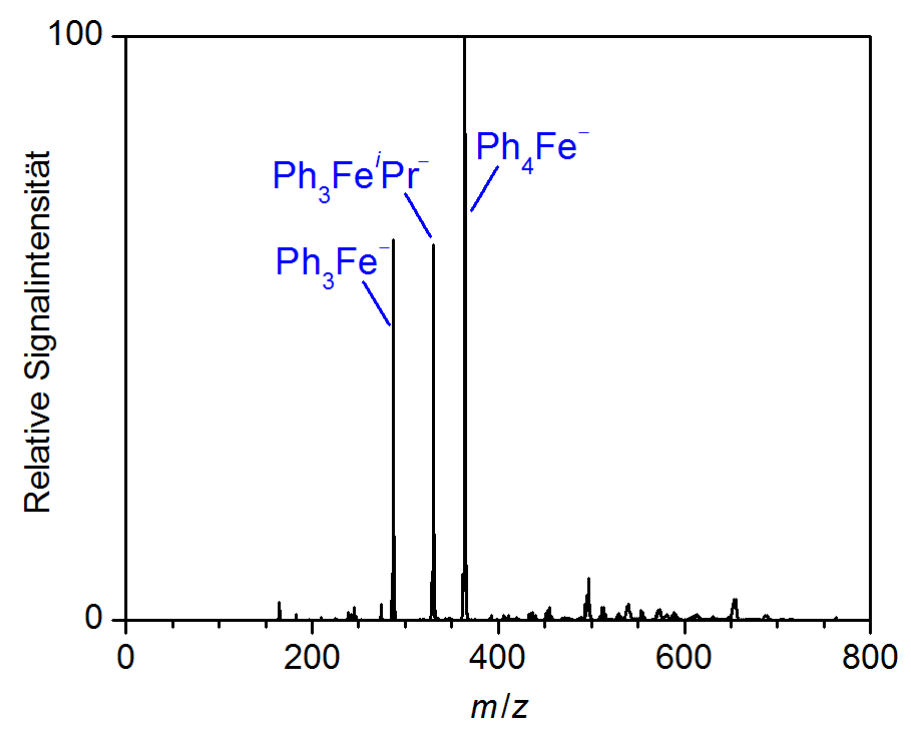

Abbildung 49. ESI-Massenspektrum (negativer lonenmodus) der Produkte aus der Reaktion von $\mathrm{Fe}(\mathrm{acac})_{3}$ (10 mM) mit TMEDA (4.0 Äq.), PhMgCl (4.0 Äq.) und 'PrCl (0.5 Äq.) in THF.

\footnotetext{
' Auf die Reaktion der in situ erzeugten Phenylferrate mit $\mathrm{EtCl}$ wurde aufgrund dessen gasförmigen Aggregationszustandes und der damit einhergehenden erschwerten Handhabung verzichtet.
} 
In einigen Fällen konnten zusätzlich Spezies des Typs $\mathrm{Ph}_{4} \mathrm{FeR}^{\prime-}$ in geringer Signalintensität ausgemacht werden. Um zu überprüfen, ob diese als $\mathrm{Ph}_{4} \mathrm{Fe}(\mathrm{IV}) \mathrm{R}^{\prime-}$ oder in der alternativen Konstitution $\mathrm{Ph}_{2} \mathrm{Fe}\left(\eta^{6}-\mathrm{Ph}_{2}\right) \mathrm{R}^{\prime-}$ mit Eisen(II)-Zentrum vorlagen, wurde den entsprechenden Reaktionslösungen 4,4'-Dimethylbiphenyl (0.5 Äq.) zugegeben. Falls ein $\pi$-gebundenes Ligandensystem vorlag, sollte ein schneller Austausch mit dem letztgenannten Substrat erfolgen. Jedoch konnte in keinem Fall solch ein Austausch beobachtet werden, sodass schlussfolgernd Eisen(IV)-Spezies anzunehmen waren. Eine Erhöhung der eingesetzten Äquivalente an R'X (1.0 Äq., 10.0 Äq.) führte zur alleinigen Dominanz rein anorganischer MgSpezies. Repräsentativ für alle betrachteten Systeme soll die Reaktion von $\mathrm{Fe}(\mathrm{acac})_{3}$ mit TMEDA (4.0 Äq.), $\mathrm{PhMgCl}$ (4.0 Äq.) und $\operatorname{PrCl}$ (0.5 Äq.) stehen, für die eine hohe Intensität des Peaks für $\mathrm{Ph}_{3} \mathrm{Fe} \mathrm{Pr}^{-}$detektiert werden konnte ( $\rightarrow$ Abbildung 49 ). Eine kinetische Betrachtung dieser Reaktionsmischung mittels ESI-Massenspektrometrie zeigte, dass die Bildung von $\mathrm{Ph}_{3} \mathrm{Fe}^{i} \mathrm{Pr}^{-}$mit der Reaktion der Eisen(II)-Spezies $\mathrm{Ph}_{3} \mathrm{Fe}^{-}$einherging ( $\rightarrow$ Abbildung 50 ). Für die letztgenannte Verbindung konnte mit $t_{1 / 2}=180 \pm 40 \mathrm{~s}$ eine Halbwertszeit ermittelt werden, die in Relation zu dem entsprechendem Experiment in Abwesenheit von ${ }^{i} \mathrm{PrCl}\left(t_{1 / 2}=580 \pm 60 \mathrm{~s}\right)$ deutlich geringer ausfiel. Hingegen blieb $\mathrm{Ph}_{4} \mathrm{Fe}^{-}$mit Eisen(III)-Kern im betrachteten Messzeitraum zeitlich stabil. Interessant war, dass die spezifische elektrische Leitfähigkeit einer analogen Reaktionslösung nach Zugabe von ${ }^{i} \mathrm{PrCl}$ zunächst anstieg und anschließend sehr rasch abfiel ( $\rightarrow$ Abbildung 51). Für die lonen in Lösung konnte aus dem zeitlichen Verlauf von $\kappa$ grafisch eine Halbwertszeit von $t_{1 / 2}=340 \pm 60 \mathrm{~s}$ ermittelt werden.

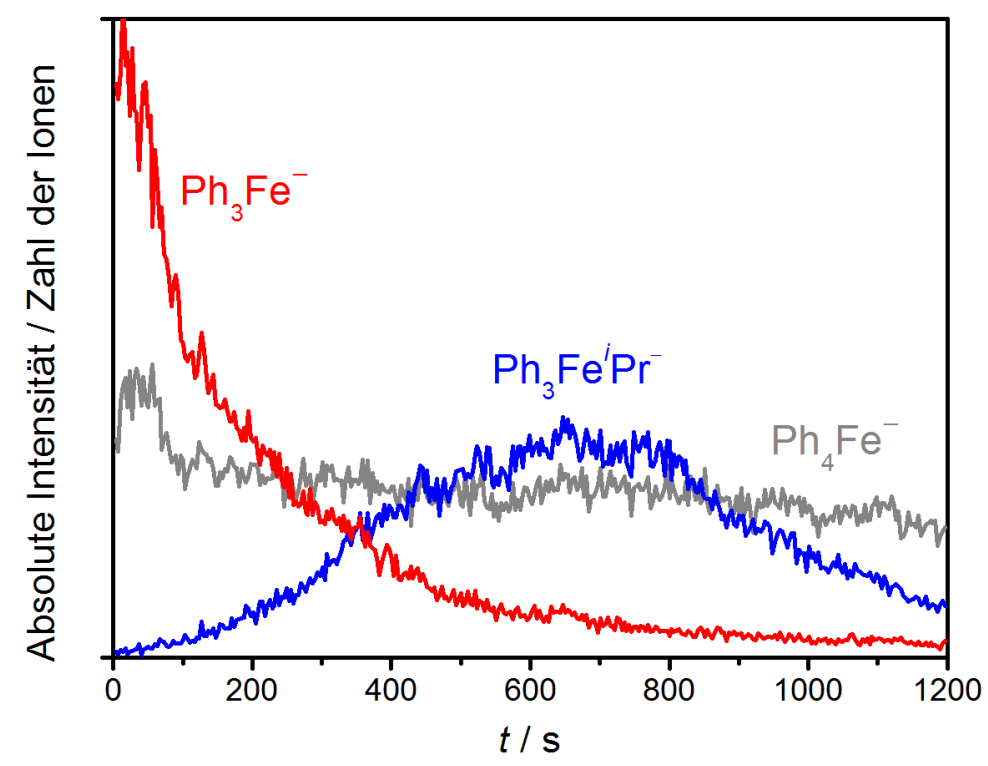

Abbildung 50. Zeitliche Entwicklung der absoluten ESI-Signalintensitäten der Anionen $\mathrm{Ph}_{3} \mathrm{Fe}^{-}, \mathrm{Ph}_{3} \mathrm{Fe}^{i} \mathrm{Pr}^{-}$und $\mathrm{Ph}_{4} \mathrm{Fe}^{-}$, die durch die Reaktion von $\mathrm{Fe}(\mathrm{acac})_{3}(10 \mathrm{~mm})$ mit TMEDA (4.0 Äq.), PhMgCl (4.0 Äq.) und 'PrCl (0.5 Äq.) in THF gebildet wurden. 


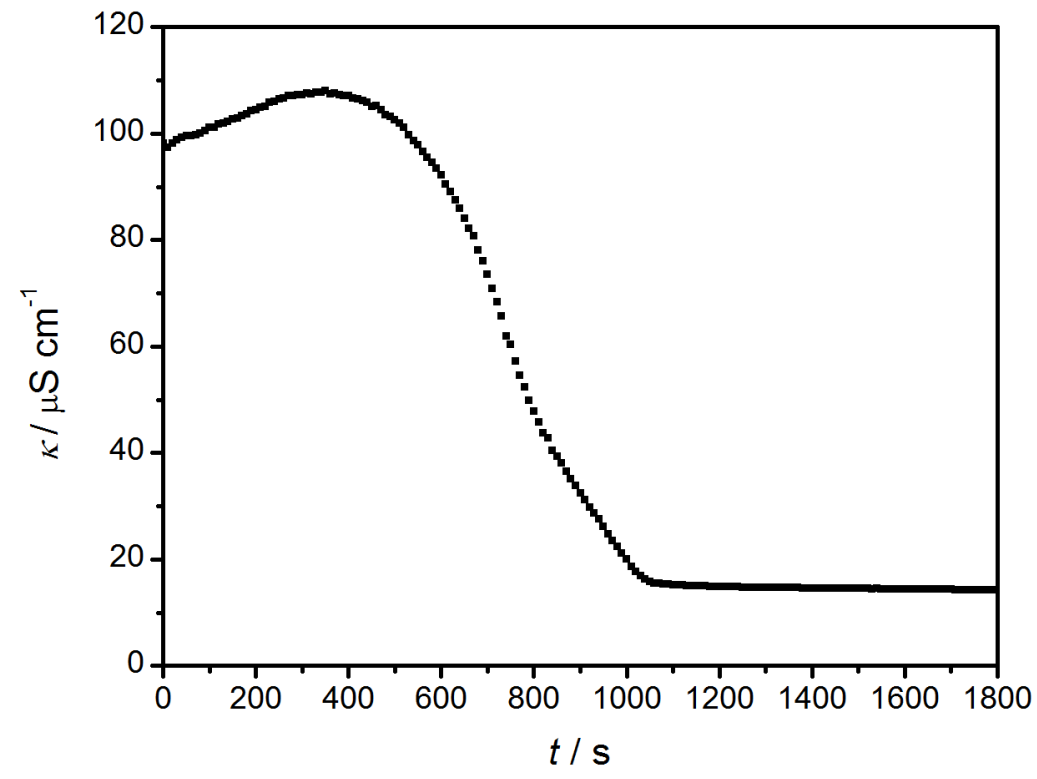

Abbildung 51. Zeitliche Entwicklung der spezifischen, elektrischen Leitfähigkeit einer Lösung von $\mathrm{Fe}(\mathrm{acac})_{3}$ (50 mM), TMEDA (4.0 Äq.) und PhMgCl (4.0 Äq.) in THF nach Zugabe von ${ }^{\mathrm{PrCl}}(0.5 \mathrm{Äq}$.) bei $T=273 \mathrm{~K}$. Zu beachten ist, dass der Startpunkt bei $t=0 \mathrm{~min}$ nicht dem Startpunkt des analogen ESI-massenspektrometrischen Experimentes ( $\rightarrow$ Abbildung 50) entspricht, da in diesem nach Zugabe von ${ }^{~} \mathrm{PrCl}$ etwas Zeit $(t<1 \mathrm{~min})$ benötigt wurde, um die Probenlösung zu entnehmen und in das Massenspektrometer zu injizieren.

Um nachzuweisen, dass die Reaktion von $\mathrm{PhMgCl}$ mit ${ }^{\mathrm{i}} \mathrm{PrCl}$ in Anwesenheit von $\mathrm{Fe}(\mathrm{acac})_{3}$ auch synthetische Relevanz hat, wurden die entsprechende Eisen-katalysierte Kreuzkupplungsreaktion im synthetischem Maßstab durchgeführt und die entstandenen Reaktionsprodukte mittels NMR-Spektroskopie analysiert ( $\rightarrow$ Abbildung 52 ).

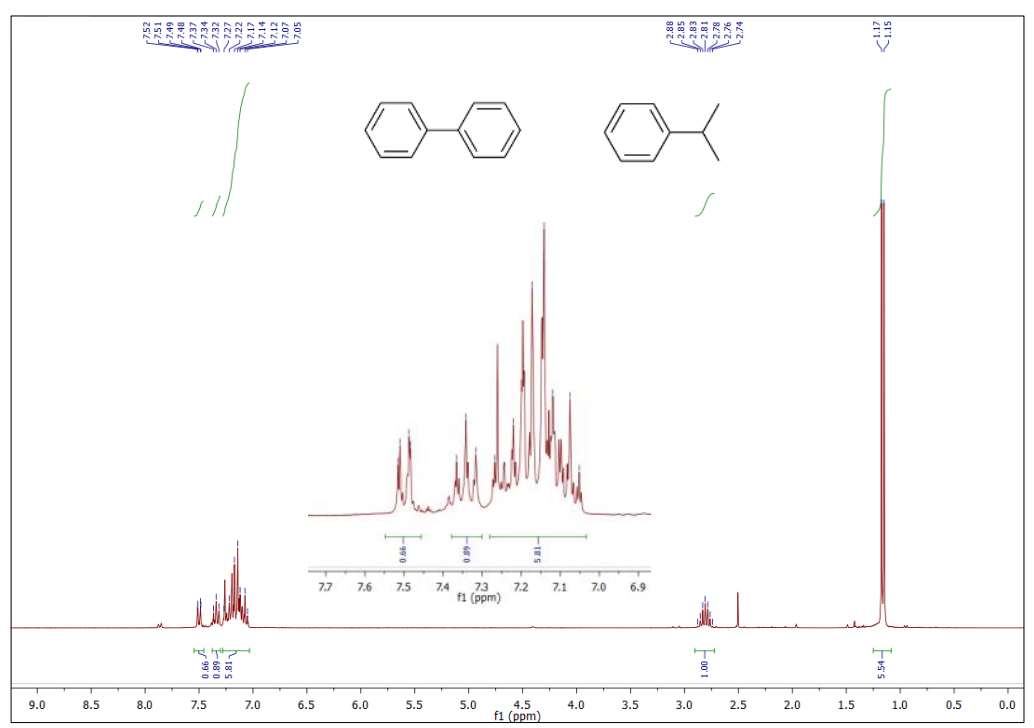

Abbildung 52. ${ }^{1} \mathrm{H}-\mathrm{NMR}$-Spektrum der Produkte, die durch die Eisen-katalysierte Kreuzkupplung von $\mathrm{PhMgCl}$ und i $\mathrm{PrCl}$ entstanden waren. Im Ausschnitt ist der Bereich der aromatischen Wasserstoffatome vergrößert dargestellt. 
Die experimentellen Daten aus dem NMR-Experiment ließen sich dem Kreuzkupplungs- und dem Homokupplungsprodukt zuordnen. Eine Ausbeute von $67 \%$ an Kreuzkupplungsprodukt (einhergehend mit $11 \%$ an Homokupplungsprodukt) belegte, dass das gewählte Modellsystem synthetisch brauchbare Ausbeuten lieferte. In einem weiteren Experiment wurden auch die Phenylferrate, die über Reaktion von $\mathrm{FeCl}_{2}(\mathrm{dppbz})_{2}$ mit $\mathrm{PhMgCl}$ (4.0 Äq.) entstanden waren (siehe Kapitel 4.1.2.3), hinsichtlich ihrer Reaktivität gegenüber ${ }^{i} \mathrm{PrCl}$ charakterisiert. Erneut konnte $\mathrm{Ph}_{3} \mathrm{Fe}^{i} \mathrm{Pr}^{-}$in hoher Signalintensität gebildet werden. Daneben war mit dem Anion ${ }^{\prime} \mathrm{PrFe}(\mathrm{dppbz})_{2}{ }^{-}$ein weiteres Reaktionsprodukt erkennbar. Zusätzlich wurden auch hier kinetische Messungen mittels ESI-Massenspektrometrie durchgeführt, in denen die Signalintensitäten verschiedener niedervalenter Phenylferrate zeitlich verfolgt wurden. Nach Abzug des thermischen Zerfalls, der in Abwesenheit von ${ }^{i} \mathrm{PrCl}$ aufgenommen wurde, zeigte sich, dass die Anionen $\mathrm{Fe}(\mathrm{dppbz})^{-}, \mathrm{PhFe}(\mathrm{dppbz})^{-}$und $\mathrm{Ph}_{2} \mathrm{Fe}(\mathrm{dppbz})^{-}$in der Präsenz dieses Substrates deutlich schneller an Signalintensität verloren.

Zusätzlich wurden Experimente durchgeführt, in denen aromatische Substrate als Reaktionspartner für die Phenylferrate dienten. Neben $p$-TolX $(X=\mathrm{Cl}, \mathrm{Br}, \mathrm{I}, \mathrm{OTf})$ wurden $o-\mathrm{CF}_{3}-\mathrm{C}_{6} \mathrm{H}_{4} \mathrm{Br}$ und $\mathrm{MesBr}$ als organische Substrate eingesetzt. Außer für $\mathrm{R}^{\prime} \mathrm{X}=p$ - $\mathrm{TolBr}$ wurde in allen Fällen erneut die heteroleptische Spezies $\mathrm{Ph}_{3} \mathrm{Fe}(\mathrm{III}) \mathrm{R}^{-}$in sehr geringen Signalintensitäten gebildet. Zusätzlich waren in zwei Fällen ( $\mathrm{R}^{\prime} \mathrm{X}=p$-Toll, $0-\mathrm{CF}_{3}-\mathrm{C}_{6} \mathrm{H}_{4} \mathrm{Br}$ ) Anionen des Typs $\mathrm{Ph}_{4} \mathrm{FeR}^{\prime-}$ detektierbar. Abermals konnte durch Zugabe von 4,4'-Dimethylbiphenyl (0.5 Äq.) zu den entsprechenden Reaktionslösungen das Vorliegen von $\mathrm{Ph}_{2} \mathrm{Fe}\left(\eta^{6}-\mathrm{Ph}_{2}\right) \mathrm{R}^{\prime-}$ ausgeschlossen und damit gleichzeitig die Präsenz von $\mathrm{Ph}_{4} \mathrm{Fe}(\mathrm{IV}) \mathrm{R}^{\prime-}$ bestätigt werden. 


\subsubsection{Reaktivität von Methylferraten gegenüber R'X}

Wurden Reaktionslösungen aus $\mathrm{Fe}(\mathrm{acac})_{3}$ und $\mathrm{MeMgBr}$ (4.0 Äq.) mit Isopropylhalogeniden iPrX (0.5 Äq.; $\mathrm{X}=\mathrm{Cl}, \quad \mathrm{Br}, \quad$ I) versetzt, blieb $\mathrm{Me}_{12} \mathrm{Fe}_{8}{ }^{-}$die dominierende Spezies ( $\rightarrow$ Abbildung 53). Daneben konnten weitere artverwandte Cluster-Anionen mit Eisen in durchschnittlichen Oxidationszahlen von 1.3 bis 1.5 in geringer Signalintensität detektiert werden $\left(\rightarrow\right.$ Tabelle 9). Die entsprechenden Reaktionen mit Arylhalogeniden $R^{\prime} X\left(R^{\prime}=P h\right.$, $p$-Tol; $\mathrm{X}=\mathrm{Cl}, \mathrm{Br}, \mathrm{I})$ führten zur Bildung identischer Cluster-Anionen. Zusätzlich wurden in Gegenwart von R'Br oder R'I Eisen-Spezies des Typs $\mathrm{Me}_{12-n} \mathrm{Fe}_{8} \mathrm{R}_{n}^{\prime}(n=1,2)$ beobachtet.

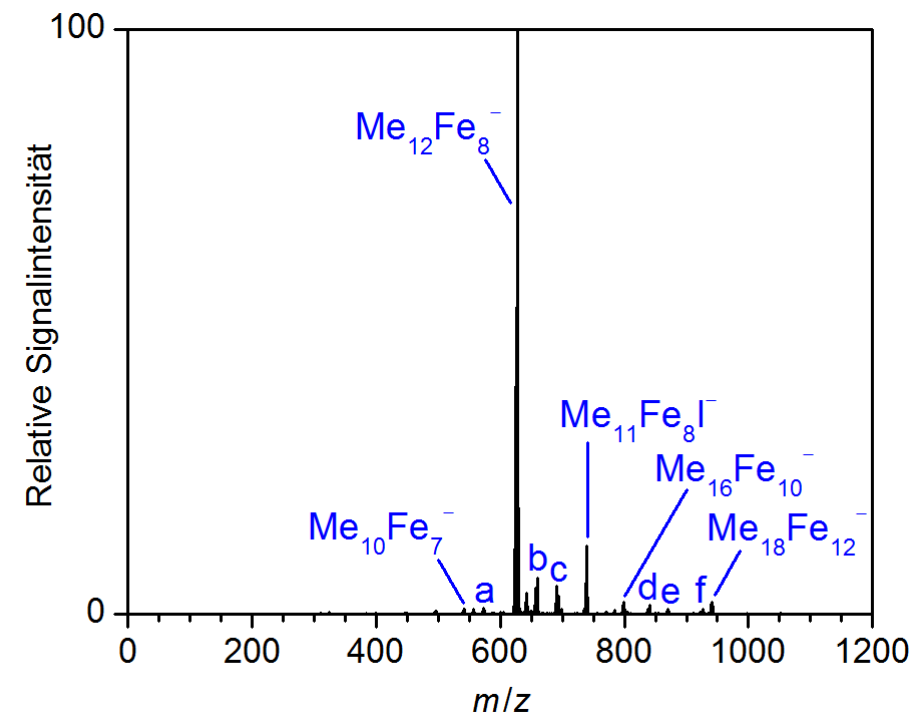

Abbildung 53. ESI-Massenspektrum (negativer lonenmodus) der Produkte aus der Reaktion von $\mathrm{Fe}(\mathrm{acac})_{3}$ (10 mM) mit MeMgBr (4.0 Äq.) und 'Prl (0.5 Äq.) in THF; $\mathrm{a}=\left[\mathrm{Me}_{7}, \mathrm{Fe}_{10}, \mathrm{O}_{2}\right]^{-}, \mathrm{b}=\left[\mathrm{Me}_{12}, \mathrm{Fe}_{8}, \mathrm{O}_{2}\right]^{-}, \mathrm{c}=\left[\mathrm{Me}_{12}, \mathrm{Fe}_{8}, \mathrm{O}_{4}\right]^{-}$, $\mathrm{d}=\mathrm{Me}_{15} \mathrm{Fe}_{11^{-}}, \mathrm{e}=\mathrm{Me}_{17} \mathrm{Fe}_{11^{-}}, \mathrm{f}=\mathrm{Me}_{17} \mathrm{Fe}_{12}{ }^{-}$.

Tabelle 9. Homoleptische Methyleisen-Cluster-Anionen gebildet in der Reaktion von $\mathrm{Fe}(\mathrm{acac})_{3} \mathrm{mit} \mathrm{MeMgBr}$ (4.0 Äq.) und $\operatorname{PrX}(0.5$ Äq., $\mathrm{X}=\mathrm{Cl}, \mathrm{Br}, \mathrm{I}$ ) in THF inklusive ihrer durchschnittlichen Oxidationszahlen.

\begin{tabular}{cc}
\hline Cluster-Anion & $\begin{array}{c}\text { Durchschnittliche } \\
\text { Oxidationszahl }\end{array}$ \\
\hline $\mathrm{Me}_{10} \mathrm{Fe}_{7^{-}}$ & 1.3 \\
$\mathrm{Me}_{12} \mathrm{Fe}_{8}^{-}$ & 1.4 \\
$\mathrm{Me}_{13} \mathrm{Fe}_{8^{-}}$ & 1.5 \\
$\mathrm{Me}_{16} \mathrm{Fe}_{10^{-}}$ & 1.5 \\
$\mathrm{Me}_{15} \mathrm{Fe}_{11^{-}}$ & 1.3 \\
$\mathrm{Me}_{17} \mathrm{Fe}_{11^{-}}$ & 1.5 \\
$\mathrm{Me}_{17} \mathrm{Fe}_{12^{-}}$ & 1.3 \\
$\mathrm{Me}_{18} \mathrm{Fe}_{12^{-}}$ & 1.4 \\
\hline
\end{tabular}


Neidig und Mitarbeiter konnten in der Vergangenheit zeigen, dass die Eisen-katalysierte Kreuzkupplungsreaktion von $\mathrm{Me}_{12} \mathrm{Fe}_{8}{ }^{-}$mit $\beta$-Bromstyrol nur durch weiteren Zusatz von $\operatorname{MeMgBr}$ (1.25 Äq. in Bezug auf $\beta$-Bromstyrol) effizient ablief. ${ }^{[39 d]}$ Eine Probenlösung, die auf Grundlage desselben Reaktionsprotokolls erstellt wurde, offenbarte keine anionischen EisenSpezies neben $\mathrm{Me}_{12} \mathrm{Fe}_{8}{ }^{-}$. Dies legt die Vermutung nahe, dass hier neutrale Reaktionsintermediate eine entscheidende Rolle spielen, die mittels ESIMassenspektrometrie nicht nachweisbar waren.

Eine gängige Methode, um Neutralspezies mit ESI-Massenspektrometrie sichtbar zu machen, ist der Einsatz von Substraten mit einem permanent geladenen Substituenten. Durch Reaktion solcher Verbindungen mit den Neutralteilchen ist es möglich, eine permanente Ladung in die neutralen Spezies zu implementieren. Anschließend ist ein Nachweis mittels ESIMassenspektrometrie möglich. Diese Methode konnte in der Vergangenheit von verschiedenen Forschungsgruppen bereits erfolgreich angewendet werden, um metallorganische Reaktionsintermediate nachzuweisen. ${ }^{[87]}$ Dies geschah jedoch unter nicht vergleichbaren Reaktionsbedingungen in Abwesenheit hochreaktiver Grignard-Reagenzien. Um neutrale Eisen-Spezies, die im Rahmen der Eisen-katalysierten Kreuzkupplung von $\mathrm{MeMgBr}$ mit Organylhalogeniden gebildet wurden, zu identifizieren, wurden ein aliphatisches und ein aromatisches elektrophiles Substrat synthetisiert, die durch die Anwesenheit von Phosphonium-Gruppen jeweils eine permanente Ladung trugen ( $\rightarrow$ Schema 14 ).
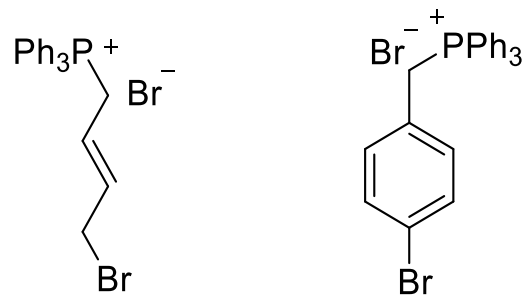

Schema 14. Strukturen der durch Phosphonium-Gruppen permanent geladenen organischen Substrate.

Die Reaktion der in situ geformten Methylferrate mit den permanent geladenen, organischen Substraten $\mathrm{R}^{\prime} \mathrm{Br}$ (0.5 Äq.) führte in beiden Fällen zur Bildung des methylierten Kreuzkupplungsproduktes $\left[\mathrm{R}^{\prime} \mathrm{Me}\right]^{+}$. Das allylische Substrat reagierte sehr schnell und war nach kurzer Zeit $(t<2 \mathrm{~min})$ vollständig verbraucht. Das aromatische Substrat hingegen reagierte deutlich langsamer. So war in diesem Fall neben dem Kreuzkupplungsprodukt [R'Me] ${ }^{+}$auch noch das intakte Substrat $\left[\mathrm{R}^{\prime} \mathrm{Br}\right]^{+}$nachweisbar ( $\rightarrow$ Abbildung 54). Das gleichzeitige Auftreten von Produkt und Edukt verdeutlichte die kurze Lebensspanne der Reaktionsintermediate, die nur in sehr geringen, nicht nachweisbaren Konzentrationen vorlagen. Der isolierte Feststoff, der 
bei der Transmetallierung mit MeMgBr in Gegenwart von TMEDA typischerweise entstand, zeigte als Suspension in THF keinerlei Reaktivität gegenüber diesen Substraten.

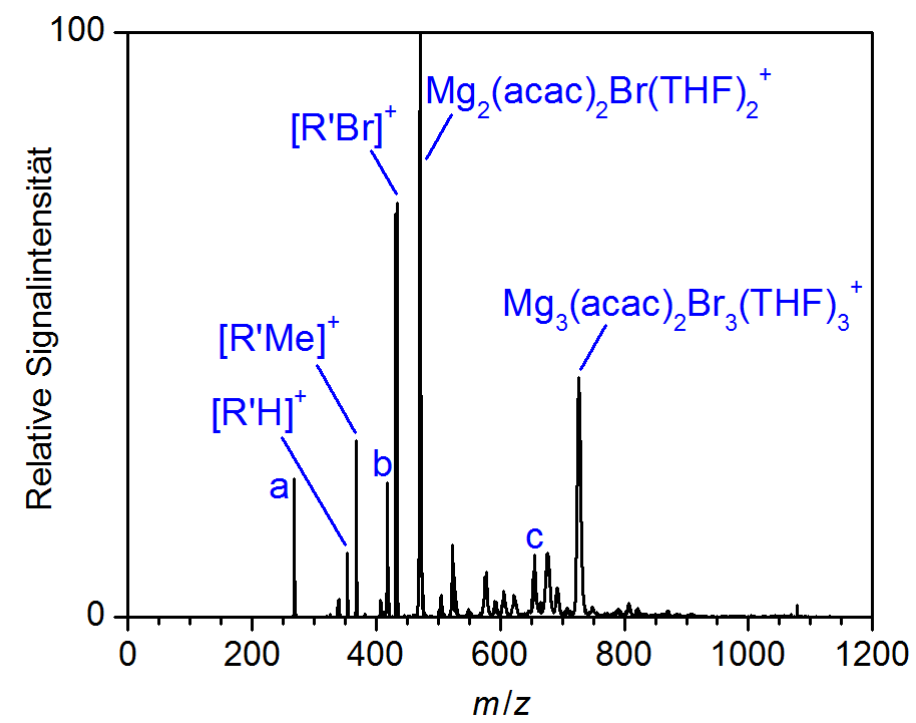

Abbildung 54. ESI-Massenspektrum (positiver lonenmodus) der Produkte aus der Reaktion von Fe(acac) 3 (10 mM) mit $\mathrm{MeMgBr}$ (4.0 Äq.) und (4-Bromobenzyl)-triphenylphosphoniumbromid (0.5 Äq.) in THF; $a=M g(a c a c)(T H F) 2^{+}$, $\mathrm{b}=\mathrm{Mg}_{2}(\mathrm{acac})_{3}(\mathrm{THF})^{+}, \mathrm{C}=\mathrm{Mg}_{3}(\mathrm{acac})_{2} \mathrm{Br}_{3}(\mathrm{THF})_{2}{ }^{+}$.

Weiterhin wurden die Reaktionen der Methylferrate mit $\mathrm{I}_{2}, \mathrm{O}_{2}$ oder $\mathrm{H}_{2} \mathrm{O}$ (jeweils 0.5 Äq.) untersucht. Die Reaktion mit $\mathrm{I}_{2}$ lieferte keine neuen Eisen-Spezies. Im Gegensatz dazu konnten in Anwesenheit von Sauerstoff neben $\mathrm{Me}_{12} \mathrm{Fe}_{8}{ }^{-}$die Anionen $\left[\mathrm{Me}_{8}, \mathrm{Fe}_{6}, \mathrm{O}_{2}\right]^{-}$und $\left[\mathrm{Me}_{9}, \mathrm{Fe}_{7}, \mathrm{O}_{2}\right]^{-}$als mögliche Deaggregationsprodukte sowie $\left[\mathrm{Me}_{12}, \mathrm{Fe}_{8}, \mathrm{O}_{2}\right]^{-}$als Oxidationsprodukt in geringer Signalintensität identifiziert werden. Gleichzeitig zeigte sich eine sehr schnell abfallende Signalintensität für den Peak des Organoferrates $\mathrm{Me}_{12} \mathrm{Fe}_{8}{ }^{-}$, was die hohe $\mathrm{O}_{2}$-Sensitivität von dieser Spezies bestätigte. ${ }^{[39 d]}$ In Gegenwart von $\mathrm{H}_{2} \mathrm{O}$ konnten keine stabilen ESI-Massenspektren im negativen lonenmodus aufgenommen werden. 


\subsubsection{Reaktivität von Butylferraten gegenüber $R^{\prime} X$}

Butylferrate wurden in ihrer Reaktivität gegenüber Phenylhalogeniden $\mathrm{PhX}(0.5$ Äq.; $\mathrm{X}=\mathrm{Cl}$, $\mathrm{Br}$, I) und $p$-Tolylhalogeniden $p$-TolX (0.5 Äq.; X $=\mathrm{Cl}, \mathrm{Br}$, I, OTf) charakterisiert. In nahezu allen Fällen (außer für $X=\mathrm{OTf}$ ) konnte die Bildung der Spezies $\mathrm{Bu}_{2 n} \mathrm{Fe}_{n}(\mathrm{II}) \mathrm{X}^{-}(n=1,2)$ beobachtet werden. Wurden $\mathrm{Phl}$ oder $p$-Toll eingesetzt, dominerte $\mathrm{Bu}_{2} \mathrm{Fe}(\mathrm{II}) \mathrm{I}^{-}$das resultierende Spektrum. Interessant war die Bildung der Spezies $\left.\mathrm{Bu}_{4} \mathrm{Fe}_{2} \mathrm{H}_{2}\right|^{-}$, die höchstwahrscheinlich aus zweifacher $\beta$-H-Eliminerung von $\left.\mathrm{Bu}_{6} \mathrm{Fe}_{2}\right|^{-}$resultierte. Für die Reaktion der Butylferrate mit $\mathrm{PhBr}$ oder p-TolOTf konnte die Bildung der Eisen(III)-Spezies $\mathrm{Bu}_{3} \mathrm{FePh}^{-}$bzw. $\mathrm{Bu}_{3} \mathrm{Fe}(p \text {-Tol })^{-}$festgestellt werden ( $\rightarrow$ Abbildung 55).

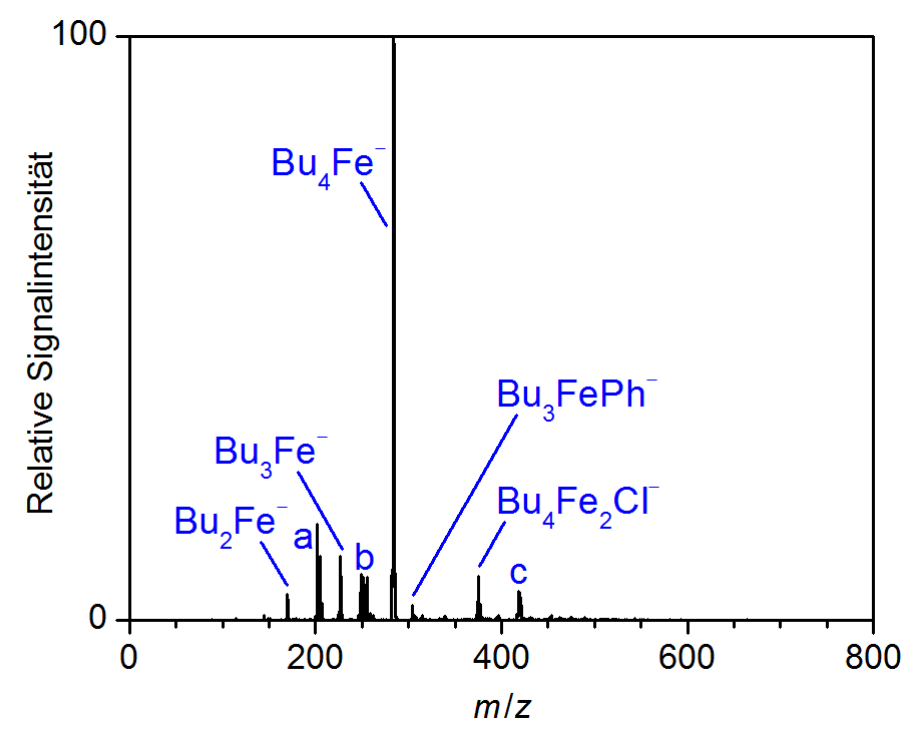

Abbildung 55. ESI-Massenspektrum (negativer lonenmodus) der Produkte aus der Reaktion von $\mathrm{Fe}(\mathrm{acac})_{3}$ (20 mM) mit BuMgCl (4.0 Äq.) und $\mathrm{PhBr}$ (0.5 Äq.) in THF; $\mathrm{a}=\left[\mathrm{Bu}_{2}, \mathrm{Fe}_{2} \mathrm{O}_{2}\right]^{-}+\mathrm{Bu}_{2} \mathrm{FeCl}^{-}, \quad b=\mathrm{Bu}_{2} \mathrm{FeBr}^{-}$, $\mathrm{C}=\mathrm{Bu}_{4} \mathrm{Fe}_{2} \mathrm{Br}^{-}$. 


\subsubsection{Reaktivität von Benzylferraten gegenüber R'X}

In situ generierte Benzylferrate wurden mit Isopropylhalogeniden ${ }^{\text {PrX }}(0.5 \mathrm{Äq}$; $\mathrm{X}=\mathrm{Cl}, \mathrm{Br}, \mathrm{I}$ ) umgesetzt. Für $\mathrm{X}=\mathrm{Cl}$ und $\mathrm{X}=\mathrm{Br}$ wurde die Spezies $\mathrm{Bn}_{3} \mathrm{Fe}(\mathrm{III})^{\prime} \mathrm{Pr}^{-}$in geringer Signalintensität beobachtet ( $\rightarrow$ Abbildung 56). Die entsprechende Reaktion mit 'Prl resultierte in der Bildung von $\mathrm{Bn}_{2} \mathrm{Fe}(\mathrm{II}) I^{-}$und $\mathrm{BnFe}(\mathrm{II}) \mathrm{I}_{2}^{-}$, deren Signalintensität deutlich zunahm, wenn die eingesetzten Äquivalente an $\operatorname{Prl}(1.0$ Äq., 2.0 Äq.) erhöht wurden.

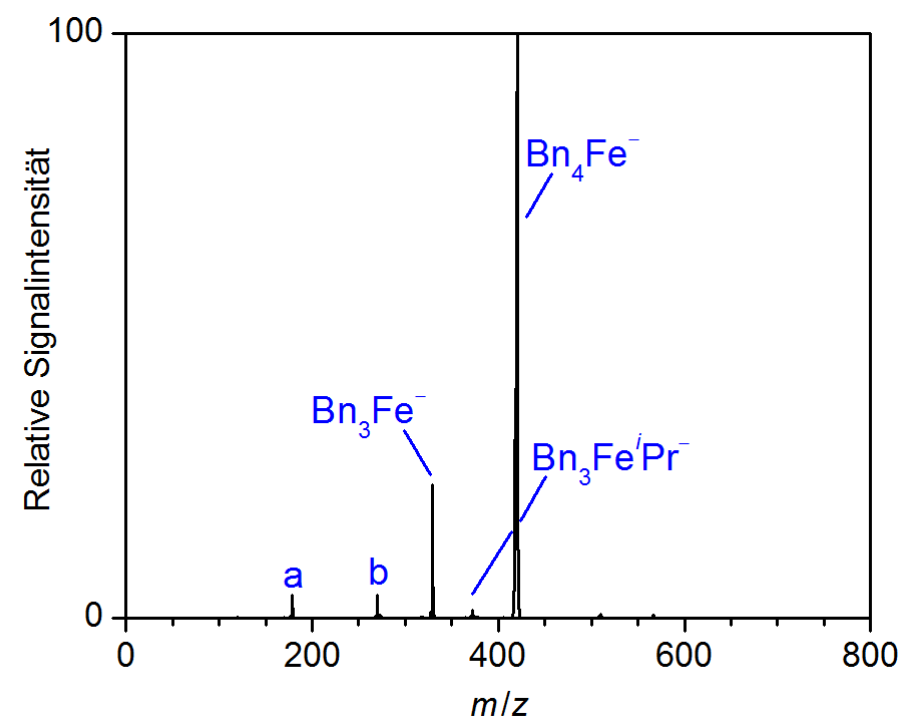

Abbildung 56. ESI-Massenspektrum (negativer lonenmodus) der Produkte aus der Reaktion von $\mathrm{Fe}(\mathrm{acac})_{3}$ (10 mM) mit BnMgCl (4.0 Äq.) und $\operatorname{PrBr}\left(0.5\right.$ Äq.) in THF; $a=\left[\mathrm{Bn}, \mathrm{Fe}, \mathrm{O}_{2}\right]^{-}, \quad b=\left[\mathrm{Bn}_{2}, \mathrm{Fe}_{,} \mathrm{O}_{2}\right]^{-}$. 


\subsubsection{Reaktivität der Organoferrate gegenüber organischen Substraten in Lösung}

Es konnte gezeigt werden, dass Phenylferrate und Benzylferrate mit Alkylhalogeniden in Lösung reagieren können. Eine Reaktion mit aromatischen (Pseudo)-Halogeniden konnte im Falle der Phenylferrate und der Butylferrate beobachtet werden. Dabei entstanden im Wesentlichen heteroleptische Spezies der allgemeinen Form $R_{3} F e(I I I) R^{\prime-}\left(R^{\prime}=A l k, A r\right)$. Eine denkbare Erklärung für die Bildung letztgenannter Spezies ist die oxidative Addition des organischen Substrates $\mathrm{R}^{\prime} \mathrm{X}$ an $\mathrm{R}_{2} \mathrm{Fe}(\mathrm{l})^{-}$gefolgt von einer Transmetallierung mit einem Äquivalent an Grignard-Reagenz ( $\rightarrow$ Schema 15). Die Tatsache, dass mit $\mathrm{Bu}_{2} \mathrm{Fe}^{-}$eine anionische Eisen(I)-Spezies detektiert werden konnte, untermauert die Möglichkeit eines solchen Reaktionspfades zur Bildung von $\mathrm{R}_{3} \mathrm{FeR}^{\prime-}$. Die hypothetische oxidative Addition kann in einer schrittweisen oder konzertierten Art und Weise auftreten. Im Falle der schrittweisen oxidativen Addition führt die homolytische Bindungsspaltung von $\mathrm{C}-\mathrm{X}$ zu einem Transfer des Halogen-Atoms auf $\mathrm{R}_{2} \mathrm{Fe}(\mathrm{I})^{-}$. Das dabei freiwerdende Organyl-Radikal kann mit der resultierenden Eisen(II)-Spezies rekombinieren und dabei das Produkt der oxidativen Addition bilden. Halogenidhaltige Anionen des Typs $\mathrm{R}_{2} \mathrm{Fe}(\mathrm{II}) \mathrm{X}^{-}$, die bei der schrittweisen oxidativen Addition als Zwischenprodukt entstehen, konnten in zahlreichen Fällen nachgewiesen werden. So traten diese nicht nur nach Reaktion mit aliphatischen Halogeniden auf. Auch in Anwesenheit aromatischer Halogenide konnten diese Intermediate erfolgreich nachgewiesen werden. Im letztgenannten Fall scheint eine Bildung über homolytische Bindungsspaltung von $\mathrm{Ar}-\mathrm{X}$ weniger wahrscheinlich, da dabei instabile Aryl-Radikale entstehen würden. Alternativ können in diesem Fall halogenidhaltige Eisen(II)-Intermediate entstehen, indem Produkte der konzertierten oxidativen Addition mit $\mathrm{R}_{3} \mathrm{Fe}(\mathrm{II})^{-}$transmetalliert werden.

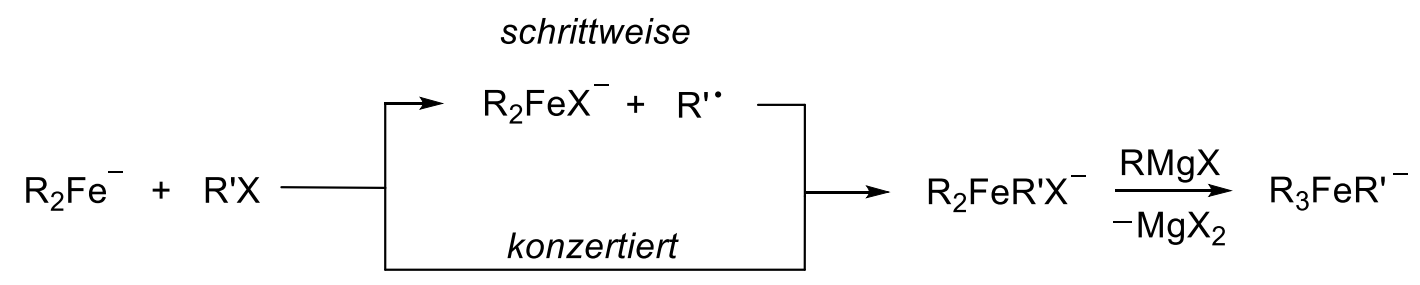

Schema 15. Möglicher Reaktionspfad zur Bildung heteroleptischer Spezies $\mathrm{R}_{3} \mathrm{FeR}^{\prime-}$ über schrittweise oder konzertierte oxidative Addition von $\mathrm{R}^{\prime} \mathrm{X}$ an $\mathrm{R}_{2} \mathrm{Fe}^{-}$mit anschließender Transmetallierung durch $\mathrm{RMgX}$.

Die heteroleptischen Spezies müssen nicht zwangsläufig über oxidative Addition an $\mathrm{R}_{2} \mathrm{Fe}(\mathrm{I})^{-}$ entstanden sein. Vorstellbar ist auch, dass zwei anionische Eisen(II)-Spezies in den Additionsprozess involviert sind und dabei $\mathrm{R}_{3} \mathrm{Fe}(\mathrm{III}) \mathrm{R}^{\prime-}$ und $\mathrm{R}_{3} \mathrm{Fe}(\mathrm{III}) \mathrm{X}^{-}$bilden ( $\rightarrow$ Schema 16 ). 
Anionen des letztgenannten Typs waren in keinem der durchgeführten Experimenten detektierbar. Wahrscheinlich ist, dass diese über Transmetallierung mit einem weiteren Äquivalent an Grignard-Reagenz direkt in $\mathrm{R}_{4} \mathrm{Fe}(\mathrm{III})^{-}$überführt werden. Eine kinetische Betrachtung der Phenylferrate in Anwesenheit von ${ }^{i} \mathrm{PrCl}$ belegte, dass $\mathrm{Ph}_{3} \mathrm{Fe}(\mathrm{II})^{-}$in Anwesenheit des aliphatischen Halogenides eine deutlich geringere Halbwertszeit aufwies (siehe Kapitel 4.2.1.1). Dies kann auf eine Reaktion von $\mathrm{Ph}_{3} \mathrm{Fe}(\mathrm{II})^{-}$mit ${ }^{i} \mathrm{PrCl}$ hindeuten. Denkbar ist aber auch, dass die Eisen(II)-Spezies nicht direkt mit ${ }^{i} \mathrm{PrCl}$ reagierte, jedoch dessen Halbwertszeit über Gleichgewichte mit reaktiven, nicht detektierbaren EisenVerbindungen indirekt beeinflusst wurde. Ein ähnlicher Sachverhalt lässt sich für $\mathrm{Fe}(\mathrm{dppbz})^{-}$, $\mathrm{PhFe}(\mathrm{dppbz})^{-}$und $\mathrm{Ph}_{2} \mathrm{Fe}(\mathrm{dppbz})^{-}$feststellen. Auch diese Eisen-Spezies wiesen in Anwesenheit von ${ }^{i} \mathrm{PrCl}$ geringere Stabilitäten auf, was aber nicht zwingend auf eine direkte Reaktion mit dem organischen Substrat hindeuten muss.

$$
2 \mathrm{R}_{3} \mathrm{Fe}^{-} \stackrel{\mathrm{R}^{\prime X}}{\longrightarrow} \mathrm{R}_{3} \mathrm{FeR}^{\prime^{-}}+\mathrm{R}_{3} \mathrm{FeX}^{-} \stackrel{\mathrm{RMgX}}{\longrightarrow} \mathrm{R}_{3} \mathrm{FeR}^{{ }^{-}}+\mathrm{R}_{4} \mathrm{Fe}^{-}
$$

Schema 16. Möglicher Reaktionspfad zur Bildung heteroleptischer Spezies $\mathrm{R}_{3} \mathrm{FeR} \mathrm{R}^{-}$durch oxidative Addition an zwei Äquivalente der anionischen Eisen(II)-Spezies $\mathrm{R}_{3} \mathrm{Fe}^{-}$mit anschließender Transmetallierung.

In wenigen Fällen ließ sich die Bildung Eisen(IV)-haltiger Spezies in sehr geringer Signalintensität beobachten. Diese können aus einer Redoxdisproportionierung entstanden sein. Analoge Überlegungen wurden bereits von Fürstner und Mitarbeiter getroffen, die eine Redoxdisproportionierung für die Bildung einer neutralen Tetraalkyleisen-Spezies annahmen. ${ }^{[88]}$

Für Methylferrate scheinen andere Additionsprozesse eine entscheidende Rolle zu spielen. Durch den Einsatz von organischen Substraten mit permanent geladenen Gruppen konnten die Kreuzkupplungsprodukte durch dauerhafte Implementierung einer kationischen Funktionalität mittels ESI-Massenspektrometrie sichtbar gemacht werden. Gleichzeitig blieb $\mathrm{Me}_{12} \mathrm{Fe}_{8}{ }^{-}$von der Anwesenheit dieser Substrate unbeeinflusst, was auf einen Reaktionsmechanismus über neutrale und sehr kurzlebige Eisen-Spezies zur Bildung des Kreuzkupplungsproduktes schließen lässt. Dies deckt sich mit Annahmen von Neidig und Mitarbeiter. ${ }^{[39 g]}$ So zeigte sich, dass isolierte Kristalle von $\mathrm{Me}_{12} \mathrm{Fe}_{8}{ }^{-}$nur dann mit $\beta$-Bromstyrol als organischem Substrat reagieren und das Kreuzkupplungsprodukt bilden konnten, nachdem einer Probenlösung von $\mathrm{Me}_{12} \mathrm{Fe}_{8}{ }^{-}$in THF 1.25 Äquivalente an MeMgBr zugesetzt worden waren. Es scheint, dass das achtkernige Eisen-Aggregat als Reservoir für weitere sehr kurzlebige Eisen-Spezies dient. Hingegen reagierte $\mathrm{Me}_{12} \mathrm{Fe}_{8}{ }^{-}$mit aromatischen Halogeniden 
$\mathrm{R}^{\prime} \mathrm{X}\left(\mathrm{R}^{\prime}=\mathrm{Ph}, p\right.$-Tol; $\left.\mathrm{X}=\mathrm{Br}, \mathrm{I}\right)$ und bildete heteroleptische Anionen des Typs $\mathrm{Me}_{11} \mathrm{Fe}_{8} \mathrm{R}^{\prime-}$ und $\mathrm{Me}_{10} \mathrm{Fe}_{8} \mathrm{R}_{2}^{-}$. Da diese Eisen in einer identischen Oxidationsstufe zum Ursprungs-Ion aufweisen, ist ein oxidativer Additionsprozess hier sehr unwahrscheinlich. Denkbar ist, dass die heteroleptischen Spezies über eine Metall-Halogen-Austauschreaktion entstanden sind $(\rightarrow$ Schema 17).

$$
\mathrm{Me}_{12} \mathrm{Fe}_{8}{ }^{-}+n \mathrm{R}^{\prime} \mathrm{X} \longrightarrow \mathrm{Me}_{12-n} \mathrm{Fe}_{8} \mathrm{R}_{n}{ }^{-}+n \mathrm{MeX}
$$

Schema 17. Metall-Halogen-Austauschreaktion zur Bildung achtkerniger, heteroleptischer Methylferrate $(n=1,2)$.

Der nach Transmetallierung von $\mathrm{FeCl}_{2} / \mathrm{TMEDA}$ mit MeMgBr ausgefallene Feststoff zeigte kein katalytisches Potential in Kreuzkupplungsreaktionen. Im Gegensatz dazu konnten Bedford und Mitarbeiter in situ katalytisch wirksame Nanopartikel über Reduktion von $\mathrm{FeCl}_{2} /$ Polyethylenglycol mit $p$-TolMgBr erzeugen. ${ }^{89]}$ 


\subsubsection{Bimolekulare Gasphasenreaktivität}

Ein weiteres Ziel dieser Arbeit war, den Ursprung heteroleptischer Eisen-Spezies aufzuklären, die über Reaktion von in situ erzeugten Organoferraten mit Organylhalogeniden entstanden waren. Hierfür war eine Methode nötig, die die Analyse der spezifischen Reaktivität definierter Organoferrate gegenüber für Eisen-katalysierte Kreuzkupplungsreaktionen typischen Reaktionspartnern ermöglichte.

\subsubsection{Aufbau und Funktionsweise einer Apparatur zur Untersuchung bimolekularer Gasphasenreaktionen}

Zur Untersuchung bimolekularer Gasphasenreaktivitäten wurde im Rahmen dieser Arbeit eine Apparatur entwickelt ( $\rightarrow$ Abbildung 57), mit der es möglich war, Gasmischungen aus einem Substratgas und Helium als Betriebs- und Stoßgas für ein Quadrupol-lonenfallen-Massenspektrometer in einem definierten Mischungsverhältnis extern zu erzeugen. ${ }^{i i}$

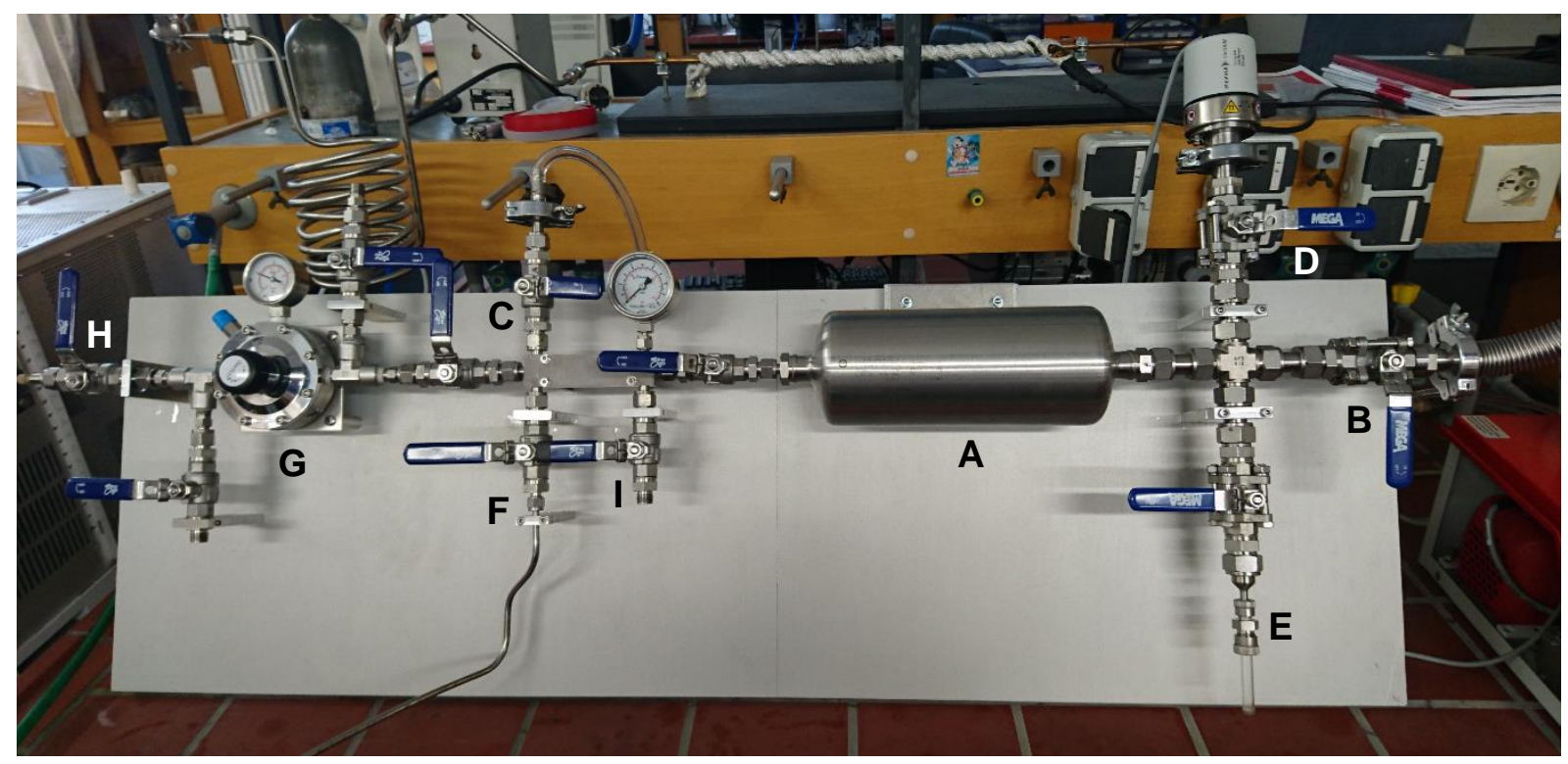

Abbildung 57. Aufbau der Apparatur zur Erzeugung von Helium-Substratgas-Mischungen: Metallkammer (A), Ventil zum Zuschalten des Turbopumpenstandes (B), Ventil zum Zuschalten der Drehschieberpumpe und zum Ablassen von Überdruck (C), Ventil zum Zuschalten der Druckmessröhre (D), Glaskolben zur Verdampfung flüssiger Substrate $(E)$, Zuleitung Helium-Gasflasche $(F)$, Druckminderer $(G)$, Ventil zum Übergang in das Massenspektrometer $(\mathrm{H})$, Anschlussmöglichkeit für gasförmige Substrate (I).

Das Herzstück dieser Apparatur bestand aus einer Edelstahl-Kammer (A) mit einem Volumen von $V=2 \mathrm{~L}$. Das Gesamtvolumen des Systems $V_{\mathrm{Ges}}=(2.5 \pm 0.1) \mathrm{L}$ wurde bestimmt, indem

\footnotetext{
ii Der Aufbau der Apparatur fand in Kooperation mit den Chemie-Werkstätten um Dipl.-Ing. V. Meyer statt. Ich danke Prof. Dr. K. Koszinowski für die Idee und die Unterstützung im gesamten Verlauf des Projektes.
} 
die gesamte Apparatur dreimal mit einem bekannten Volumen an Pentan vollständig geflutet wurde. Ausgehend von der Metallkammer führten gängige vakuum- und überdruckstabile Swagelok-Rohrverbindungen zu weiteren Elementen dieser Apparatur. Die einzelnen Bauteile waren durch Ventile voneinander getrennt. Mit einem Turbopumpenstand mit eingebauter Membranvorpumpe (Hicube 80 Eco, Pfeiffer Vacuum) konnte die gesamte Apparatur auf ein Hochvakuum von bis zu $p=10^{-6}$ mbar evakuiert werden (Zuschaltung über Ventil B). Zusätzlich war eine Drehschieberpumpe (RE 2.5, Vacuubrand) an die Apparatur angeschlossen (Zuschaltung über Ventil C). Diese wurde zur Reinigung der Apparatur nach deren Anwendung verwendet. Zusätzlich war es möglich, die Membranvorpumpe des Turbopumpenstandes zu entlasten, indem mit der Drehschieberpumpe ein davon unabhängiges Vorvakuum erzeugt wurde. Mit einer Druckmessröhre (PKR 251, Pfeiffer Vacuum), die mit einer Kombination aus einem Pirani-Element und einem KaltkathodenSystem arbeitete, gelang es, den Druck im Bereich zwischen Atmosphärendruck und Endvakuum zu bestimmen (Zuschaltung über Ventil D). Flüssige Substrate konnten über einen Glaskolben in das Vakuum der Apparatur evakuiert werden (E). Zu beachten war, dass der Kolben im Vorfeld entgast werden musste, um Kontaminationen mit Sauerstoff in der resultierenden Gasmischung zu vermeiden. Hierfür wurde das Substrat in den Kolben injiziert, mit flüssigem Stickstoff eingefroren und der Kolben dreimal über einen Zeitraum von $t=5 \mathrm{~min}$ eingelassen. Die Ventile vor dem Turbopumpenstand (B), vor der Druckmessröhre (D) und vor der Verbindung zum Glaskolben (Ventil über E) wurden während des Entgasens mehrfach geöffnet und geschlossen, um das eingeschlossene Totvakuum in diesen zu reduzieren. War der Vorgang des Entgasens beendet, wurde das Ventil zum Kolben geschlossen, das Substrat aufgetaut und dieses anschließend bei geschlossenen Pumpen-Ventilen in die Apparatur evakuiert. An der dabei auftretenden Druckveränderung konnte bestimmt werden, wann das Substrat vollständig in die Apparatur verdampft war. Anschließend wurde das Ventil zur Druckmessröhre hin geschlossen, um Schäden durch Überdruck zu vermeiden. Nun konnte die Apparatur über eine separate Zuleitung mit Helium auf $p=6$ bar aufgefüllt werden (F). Um ein genaues Einstellen des Enddruckes zu erreichen, war es von besonderer Bedeutung, dass der Druckminderer an der Helium-Gasflasche im Vorfeld keinen Restdruck aufwies. Nach Erreichen des gewünschten Enddruckes wurde die Helium-Zuleitung geschlossen, die Gasmischung zu einem Druckminderer $(G)$ und von dort aus weiter über eine PEEK-SchlauchVerbindung in das Massenspektrometer geleitet (Zugang über Ventil H). Über ein spezielles Ventil am Massenspektrometer konnte dieses nun unabhängig vom Standard-Heliumstrom mit der alternativen Gasmischung betrieben und versorgt werden. Wichtig war, dass der Ausgangsdruck ( $p=1.4$ bar) am eingebauten Druckminderer so eingestellt wurde, dass ein 
optimales Arbeiten am Massenspektrometer mit einem typischen Arbeitsdruck von $p=4 \cdot 10^{-6}$ mbar möglich war. Um für alle Experimente Vergleichbarkeit zu gewährleisten, wurde der Ausgangsdruck des Druckminderers nicht mehr verändert. Die PEEK-Zuleitung musste im Vorfeld (gesteuert über die Arbeitssoftware des Massenspektrometers) mit der Helium-Substratgas-Mischung gründlich gespült werden (mind. 10-mal), damit ein konstantes Helium-zu-Substratgas-Verhältnis im Massenspektrometer vorlag. Zusätzlich beinhaltete die Apparatur für zukünftige Anwendungen einen weiteren Zugang (I), über den die Einspeisung gasförmiger Substrate in definierten Mengen möglich wäre.

Zur Reinigung der Apparatur wurde nach Beendigung der Experimente das Massenspektrometer durch Umschalten des entsprechenden Ventils wieder mit StandardHeliumstrom versorgt. Anschließend war es möglich, die Helium-Substratgas-Mischung aus der Apparatur zu entlassen (Ventil C). Nach mehrfachem Spülen der gesamten Apparatur mit Helium (mind. 3-mal) wurde zunächst die Drehschieberpumpe zugeschaltet, bis ein Druck von $p=0.1$ mbar erreicht wurde. Anschließend wurde mit dem Turbopumpenstand evakuiert, bis sich das Vakuum einstellte, das im Vorfeld des Experimentes in der Apparatur herrschte. Abschließend wurde nach vorheriger Isolation aller Pumpen und der Druckmessröhre die Apparatur mit Helium befüllt. Über Steuerung mit der Arbeitssoftware am Massenspektrometer waren sowohl die Helium-Substratgas-Zuleitung als auch die Standard-Heliumzuleitung in genannter Reihenfolge mit reinem Helium intensiv zu spülen (mind. jeweils 10-mal), um etwaige Reste an Substratgas zu entfernen. 


\subsubsection{Methodenvalidierung}

Um die zuvor beschriebene Apparatur für die Durchführung bimolekularer GasphasenExperimente am Quadrupol-lonenfallen-Massenspektrometer zu verifizieren und deren Qualität zu überprüfen, wurden literaturbekannte Substitutionsreaktionen in der Gasphase auf qualitativer und quantitativer Ebene reproduziert. ${ }^{[00]}$ Zusätzlich wurde eine bisher in der Literatur unbekannte Substitutionsreaktion in der Gasphase untersucht. Die Reaktion von Bromid-lonen mit Methyliodid soll dabei repräsentativ für alle durchgeführten GasphasenExperimente stehen und zeigen, auf welche Art und Weise die Reproduktionen erfolgten $(\rightarrow$ Schema 18).

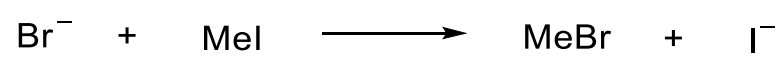

Schema 18. Bimolekulare Gasphasen-Reaktion von Bromid-lonen mit Methyliodid.

Zur Realisierung dieser Reaktion wurde eine definierte Gasmischung von Methyliodid und Helium erzeugt und in das Massenspektrometer transferiert. Anschließend wurde eine Lösung von Tetraethylammoniumbromid in THF (1 mM) erstellt und über eine gasdichte Hamiltonspritze in das ESI-Massenspektrometer injiziert. Im resultierenden Massenspektrum im negativen lonenmodus konnten im niedrigen Massenbereich Bromid-lonen beobachtet werden. Wurden diese in der lonenfalle isoliert, waren gleichzeitig lodid-lonen als Produkt der entsprechenden Substitutionsreaktion in der Gasphase erkennbar ( $\rightarrow$ Abbildung 58). Dieses Resultat bestätigte die Möglichkeit, mithilfe der entwickelten Apparatur bimolekulare Gasphasenreaktionen qualitativ am Massenspektrometer zu analysieren.

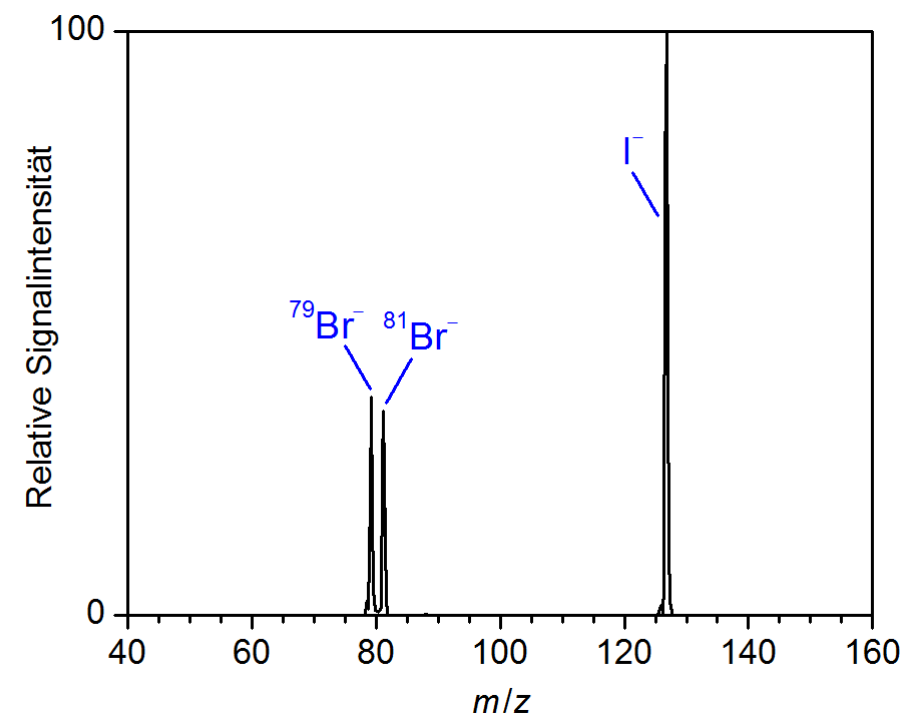

Abbildung 58. ESI-Massenspektrum (negativer lonenmodus) nach Isolation ( $m / z 80)$ von $\mathrm{Br}^{-} \mathrm{mit}^{-}$als Produkt der Ionen-Molekül-Reaktion mit Mel. 
Im nächsten Schritt wurden im Rahmen quantitativer Untersuchungen biomolekulare Geschwindigkeitsraten $k_{2}$ ermittelt. Grundlage hierfür war die schrittweise Erhöhung der Fragmentierungszeit (Zeit zwischen Fragmentierung und Detektion) isolierter Edukt-Ionen in der lonenfalle nach Einspeisung einer definierten Gasmischung aus Helium und einem neutralem Reaktanten. Dies ermöglichte eine kinetische Verfolgung der Substitutionsreaktionen in der Gasphase. In einer Auftragung der relativen Intensität der Edukt- und der Produkt-lonen gegen die Fragmentierungszeit $t$ war ein exponentieller Zusammenhang der resultierenden Datenpunkte offensichtlich. Erneut soll dies anhand der Gasphasenreaktion von Bromid-Ionen mit Methyliodid verdeutlicht werden ( $\rightarrow$ Abbildung 59 ).

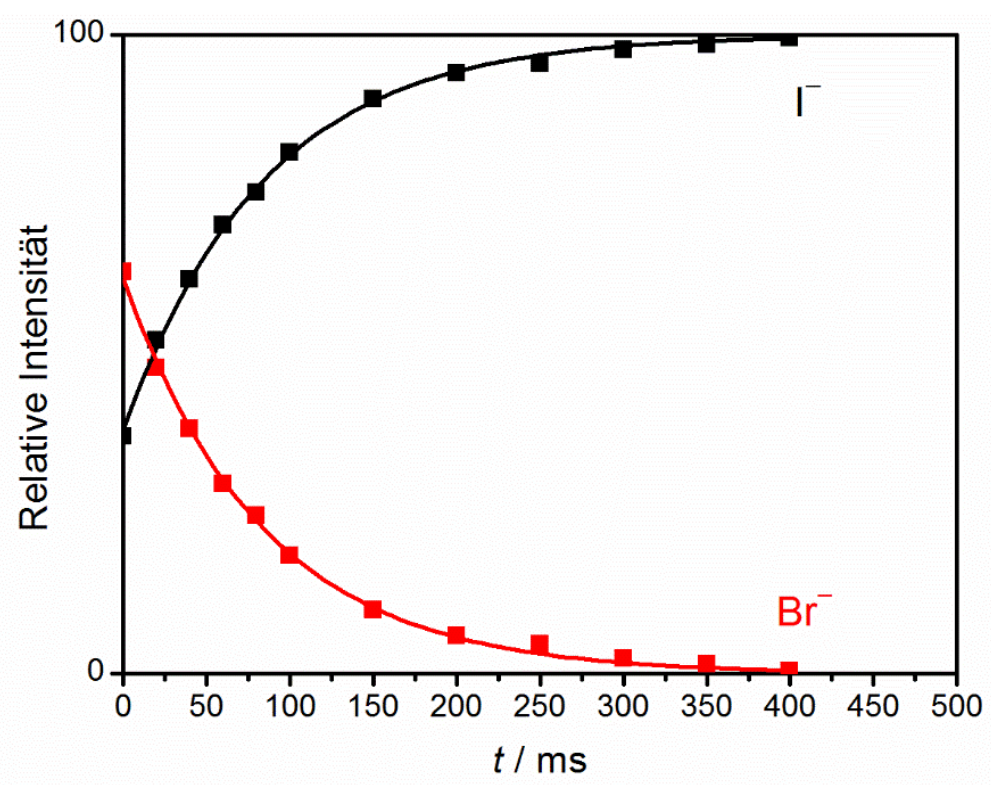

Abbildung 59. Zeitliche Entwicklung und exponentielle Anpassung der normierten relativen Signalintensitäten von $\mathrm{Br}^{-}$und $\mathrm{I}^{-}$, entstanden in der lonen-Molekül-Reaktion von Bromid-Ionen mit Methyliodid $(V=128 \mu \mathrm{L})$ in der Gasphase aufgenommen mittels ESI-Massenspektrometrie.

Da der neutrale Reaktant im Verhältnis zum geladenen Edukt im hohen Überschuss vorlag, herrschten Bedingungen pseudo-erster Ordnung. Somit waren aus den exponentiellen Verläufen dieser Auftragungen Geschwindigkeitsraten pseudo-erster Ordnung $k_{1}$ direkt zugänglich. Solche kinetischen Experimente wurden für fünf definierte Volumina an Reaktant durchgeführt und jeweils zweifach reproduziert. Aus einer Auftragung der gemittelten Geschwindigkeitsraten $k_{1}$ gegen die eingesetzte Stoffmenge $n$ an Reaktant war ein lineares Verhalten der resultierenden Datenpunkte für alle analysierten Gasphasenreaktionen augenscheinlich, so auch für die Reaktion der Bromid-Ionen mit Mel ( $\rightarrow$ Abbildung 60). 


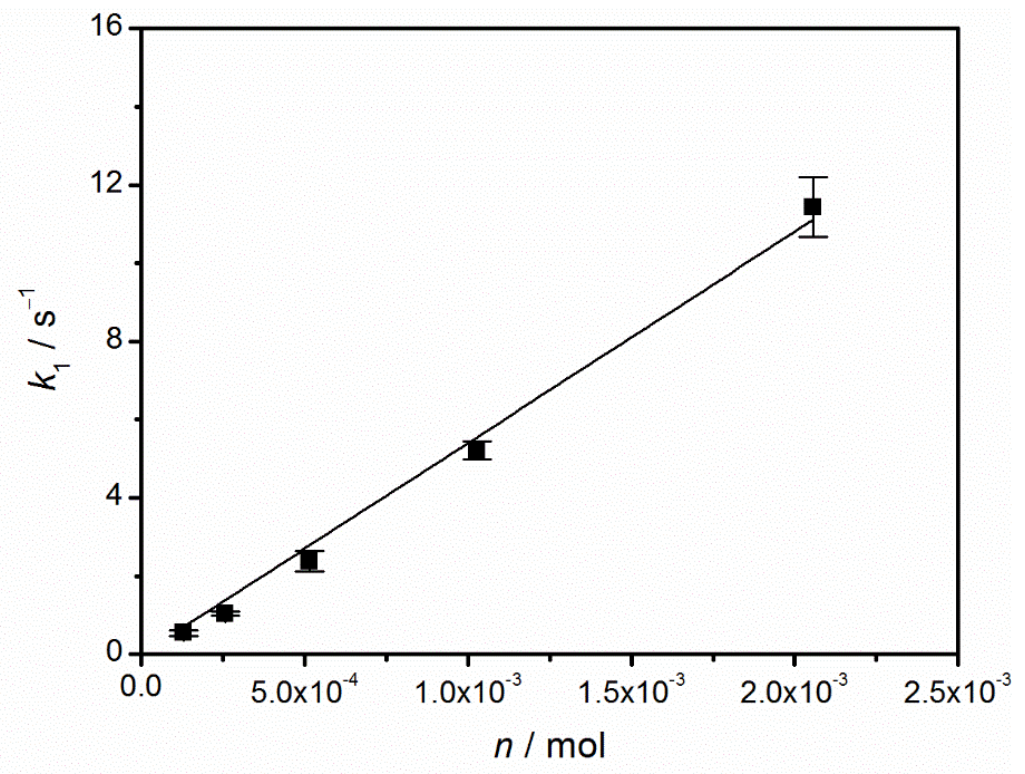

Abbildung 60. Linearer Zusammenhang der ermittelten Geschwindigkeitsraten $k_{1}$ und der Reaktantstoffmenge $n$ für die lonen-Molekül-Reaktion von $\mathrm{Br}^{-}$mit Methyliodid.

Um den absoluten Druck an Helium $p_{\mathrm{He}}$ in der lonenfalle zu ermitteln, wurden die Steigungen $m$ aus den Auftragungen $k_{1}$ gegen $n$ aller Reaktionen berücksichtigt. Aufgrund der schnelleren Diffusion von Helium im Vergleich zu den neutralen Reaktanten, musste $m$ um den Quotient aus den Wurzeln der Molmassen von Helium und dem Reaktanten jeweils korrigiert werden (Gleichung 4.2.2.2-1).[61,91]

$$
k_{\mathrm{obs}}^{\prime}=m \cdot \frac{\sqrt{\mathrm{M}_{\text {Helium }}}}{\sqrt{\mathrm{M}_{\text {Reaktant }}}}
$$

$m=$ Steigung der resultierenden Geraden aus einer Auftragung von $k_{1}$ gegen $n ; \mathrm{M}=$ Molmassen

Anschließend wurde der Quotient aus den resultierenden Werten $K_{\text {obs }}$ und den bimolekularen Geschwindigkeitsraten $k_{2, \text { Lit }}$ aus der Literatur bestimmt. ${ }^{[00]}$ Zur Veranschaulichung wurde der Mittelwert der Quotienten $m^{\prime}=(2.44 \pm 0.74) \cdot 10^{13} \mathrm{~cm}^{3} \mathrm{~mol}^{-1}$ als Steigung für die Gerade genutzt, die den linearen Zusammenhang der resultierenden Datenpunkte aus einer Auftragung von $k_{\text {obs }}^{\prime}$ gegen $k_{2, \text { Lit }}$ in logarithmischer Skalierung aufzeigen sollte $\left(\rightarrow\right.$ Abbildung 61). Nun war es möglich, mit $p_{\mathrm{He}}=0.459 \pm 0.137 \mathrm{mTorr}$ den absoluten Druck an Helium in der lonenfalle zu berechnen (Gleichung 4.2.2.2-2).

$$
p_{\mathrm{He}}=\frac{m^{\prime} \cdot T_{\mathrm{g}} \cdot V_{\mathrm{r}} \cdot p_{\mathrm{r}}}{T_{\mathrm{r}} \cdot N_{\mathrm{A}}}
$$

$m=$ Steigung der resultierenden Geraden aus einer Auftragung von $k_{\text {obs }}^{\prime}$ gegen $k_{2, L i t} ; T_{g}=$ Temperatur in der Gasphase (300 K); $V_{\mathrm{r}}=$ Volumen des Reservoirs (2.5 L); $p_{\mathrm{r}}=$ Gesamtdruck im Reservoir (6 bar); $T_{\mathrm{r}}=$ Temperatur im Reservoir (298 K); $N_{\mathrm{A}}=$ Avogadro-Konstante 


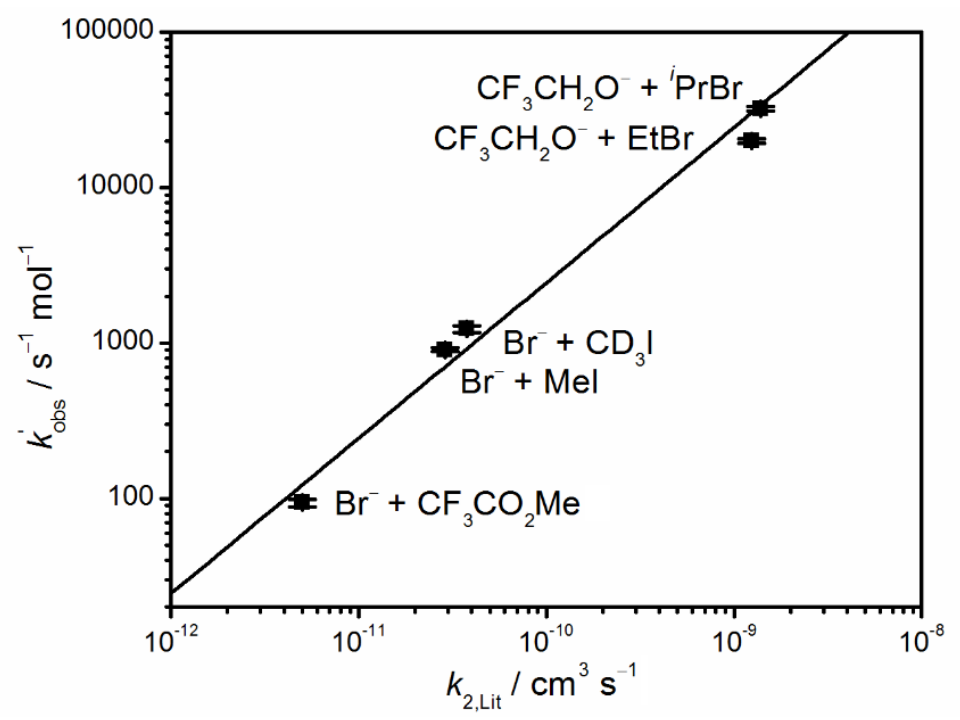

Abbildung 61. Zusammenhang und lineare Anpassung der experimentellen Geschwindigkeitsraten k'obs und der literaturbekannten bimolekularen Geschwindigkeitsraten $k_{2}$ für alle untersuchten Gasphasenreaktionen in logarithmischer Skalierung.

Zuletzt wurden die bimolekularen Geschwindigkeitsraten $k_{2}$ aller untersuchter Substitutionsreaktionen in der Gasphase ermittelt (Gleichung 4.2.2.2-3) und mit den Literaturwerten $k_{2, \text { Lit }}$ verglichen ( $\rightarrow$ Tabelle 10). Mit einer durchschnittlichen Abweichung von $\pm 30 \%$ konnten die Substitutionsreaktionen erfolgreich auf quantitativer Ebene reproduziert werden. Vergleichbare Abweichungen sind auch für andere Einlassverfahren typisch. ${ }^{[61,91]}$

$$
k_{2}=\frac{k_{\mathrm{obs}}^{\prime} \cdot T_{\mathrm{g}} \cdot V_{\mathrm{r}} \cdot p_{\mathrm{r}}}{T_{\mathrm{r}} \cdot N_{\mathrm{A}} \cdot p_{\mathrm{He}}}
$$

$k_{\text {obs }}^{\prime}=$ siehe Formel (1); $T_{g}=$ Temperatur in der Gasphase $(300 \mathrm{~K}) ; V_{r}=$ Volumen des Reservoirs $(2.5 \mathrm{~L}) ; p_{\mathrm{r}}=$ Gesamtdruck im Reservoir (6 bar); $T_{\mathrm{r}}=$ Temperatur im Reservoir $(298 \mathrm{~K}) ; N_{\mathrm{A}}=$ Avogadro-Konstante; $p_{\mathrm{He}}=$ absoluter Druck an Helium in der lonenfalle (0.459 mTorr)

Tabelle 10. Übersicht über die untersuchten lonen-Molekül-Reaktionen (IMR) mit den berechneten bimolekularen Geschwindigkeitsraten $k_{2}$, den Literaturwerten $k_{2, \text { Lit }}{ }^{[90]}$ und den entsprechenden prozentualen Abweichungen.

\begin{tabular}{lccc}
\hline \multicolumn{1}{c}{ IMR } & $\boldsymbol{k}_{\mathbf{2}} / \mathbf{c m}^{\mathbf{3}} \mathbf{s}^{-\mathbf{1}}$ & $\boldsymbol{k}_{2, \mathrm{Lit}} / \mathbf{c m}^{\mathbf{3}} \mathbf{s}^{-\mathbf{1}}$ & Abweichung $/ \%$ \\
\hline $\mathrm{CF}_{3} \mathrm{CH}_{2} \mathrm{O}^{-}+\mathrm{EtBr}$ & $(8.27 \pm 0.27) \cdot 10^{-10}$ & $(1.24 \pm 0.04) \cdot 10^{-9}$ & -33 \\
$\mathrm{CF}_{3} \mathrm{CH}_{2} \mathrm{O}^{-}+{ }^{\mathrm{P}} \mathrm{PrBr}$ & $(1.32 \pm 0.04) \cdot 10^{-9}$ & $(1.39 \pm 0.07) \cdot 10^{-9}$ & -5 \\
$\mathrm{CF}_{3} \mathrm{CH}_{2} \mathrm{O}^{-}+\mathrm{Mel}$ & $(1.19 \pm 0.04) \cdot 10^{-9}$ & - & - \\
$\mathrm{Br}^{-}+\mathrm{Mel}$ & $(3.71 \pm 0.11) \cdot 10^{-11}$ & $(2.89 \pm 0.09) \cdot 10^{-11}$ & +28 \\
$\mathrm{Br}^{-}+\mathrm{CD}_{3} \mathrm{ll}$ & $(5.03 \pm 0.27) \cdot 10^{-11}$ & $(3.78 \pm 0.09) \cdot 10^{-11}$ & +33 \\
$\mathrm{Br}^{-}+\mathrm{CF}_{3} \mathrm{CO}_{2} \mathrm{Me}$ & $(3.84 \pm 0.22) \cdot 10^{-12}$ & $(5.00 \pm 1.50) \cdot 10^{-12}$ & -23 \\
\hline
\end{tabular}




\subsubsection{Bimolekulare Gasphasenreaktivität von Phenylferraten gegenüber R'X}

Nachdem die zuvor beschriebene Methode für die qualitative und quantitative Analyse von bimolekularen Gasphasen-Reaktionen erfolgreich getestet worden war, wurde darauf aufbauend die intrinsische Reaktivität von Organoferraten gegenüber einfachen Vertretern der Organylhalogenide im Detail untersucht $(\rightarrow$ Tabelle 11). Erneut standen speziell die Phenylferrate im Fokus, da Reaktionslösungen von $\mathrm{Fe}(\mathrm{acac})_{3}$ mit $\mathrm{PhMgCl}(4.0$ Äq.) in der Präsenz von $\mathrm{PPh}_{3}$ hinreichend stabile Komplexe mit Eisen in den Oxidationsstufen $+\mathrm{l},+\mathrm{ll}$ und +III lieferten. Zusätzlich war es möglich, über stoßinduzierte Kollision mit Helium in der Gasphase in situ Phenylferrate mit Eisen(0)- oder Eisen(-I)-Zentren sowie $\mathrm{Ph}_{2} \mathrm{Fe}\left(\mathrm{I}^{-}{ }^{-} \mathrm{zu}\right.$ erzeugen. So waren mit einer einzigen Probenlösung Eisenkomplexe mit einer Vielzahl von Oxidationsstufen direkt oder indirekt zugänglich.

Tabelle 11. Ionen-Molekül-Reaktionen von ausgewählten Phenylferraten mit Organylhalogeniden inklusive der Oxidationsstufen der Eisen-Zentren.

\begin{tabular}{lcccccccc}
\hline Phenylferrat & Oxidationsstufe & Mel & Etl & PrCl & PrBr & iPrl & tBul & Phl \\
\hline $\mathrm{Ph}_{4} \mathrm{Fe}^{-}$ & $+\mathrm{lll}$ & $--^{a}$ & - & - & - & - & - & - \\
$\mathrm{Ph}_{3} \mathrm{Fe}^{-}$ & $+l l$ & - & - & - & - & - & - & - \\
$\mathrm{Ph}_{2} \mathrm{Fe}^{-}$ & $+l$ & $+^{b}$ & + & - & - & + & + & + \\
$\mathrm{Ph}_{2} \mathrm{Fe}_{(}\left(\mathrm{PPh}_{3}\right)^{-}$ & $+l$ & - & - & - & - & - & - & - \\
$\mathrm{Ph}_{3} \mathrm{Fe}_{2}\left(\mathrm{PPh}_{3}\right)^{-}$ & +1 & - & - & - & - & - & - & - \\
$\mathrm{PhFe}_{2}\left(\mathrm{PPh}_{3}\right)^{-}$ & 0 & + & + & - & - & + & + & + \\
$\mathrm{Fe}\left(\mathrm{PPh}_{3}\right)^{-}$ & -1 & + & + & - & - & + & + & - \\
\hline
\end{tabular}

${ }^{a}$ keine Reaktion. ${ }^{b}$ Reaktion zu beobachten.

Während die Spezies $\mathrm{Ph}_{4} \mathrm{Fe}(\mathrm{III})^{-}$und $\mathrm{Ph}_{3} \mathrm{Fe}(\mathrm{II})^{-}$sowie die $\mathrm{PPh}_{3}$-stabilisierten Eisen(I)-AtKomplexe $\mathrm{Ph}_{2} \mathrm{Fe}\left(\mathrm{PPh}_{3}\right)^{-}$und $\mathrm{Ph}_{3} \mathrm{Fe}_{2}\left(\mathrm{PPh}_{3}\right)^{-}$allesamt unreaktiv gegenüber Organylhalogeniden waren, reagierte die in situ generierte Eisen-Spezies $\mathrm{Ph}_{2} \mathrm{Fe}(\mathrm{I})^{-}$mit Organyliodiden zu $\mathrm{Ph}_{2} \mathrm{Fe}(\mathrm{II}) I^{-}$unter Freisetzung eines Organylradikals ( $\rightarrow$ Schema 19). Repräsentativ für diese Art der Reaktivität soll die Reaktion von $\mathrm{Ph}_{2} \mathrm{Fe}(\mathrm{I})^{-}$mit ${ }^{\mathrm{t}} \mathrm{Bul}$ stehen ( $\rightarrow$ Abbildung 62 ).

$$
\mathrm{Ph}_{2} \mathrm{Fe}^{-}+\mathrm{R}^{\prime} \mathrm{l} \longrightarrow \mathrm{Ph}_{2} \mathrm{Fel}^{-}+\mathrm{R}^{\prime}
$$

Schema 19. Bildung von $\mathrm{Ph}_{2} \mathrm{Fe}(\mathrm{II}) \mathrm{I}^{-}$unter Freisetzung eines Organylradikals durch Reaktion der Eisen(I)-Spezies $\mathrm{Ph}_{2} \mathrm{Fe}^{-}$mit Organyliodiden R'l in der Gasphase. 


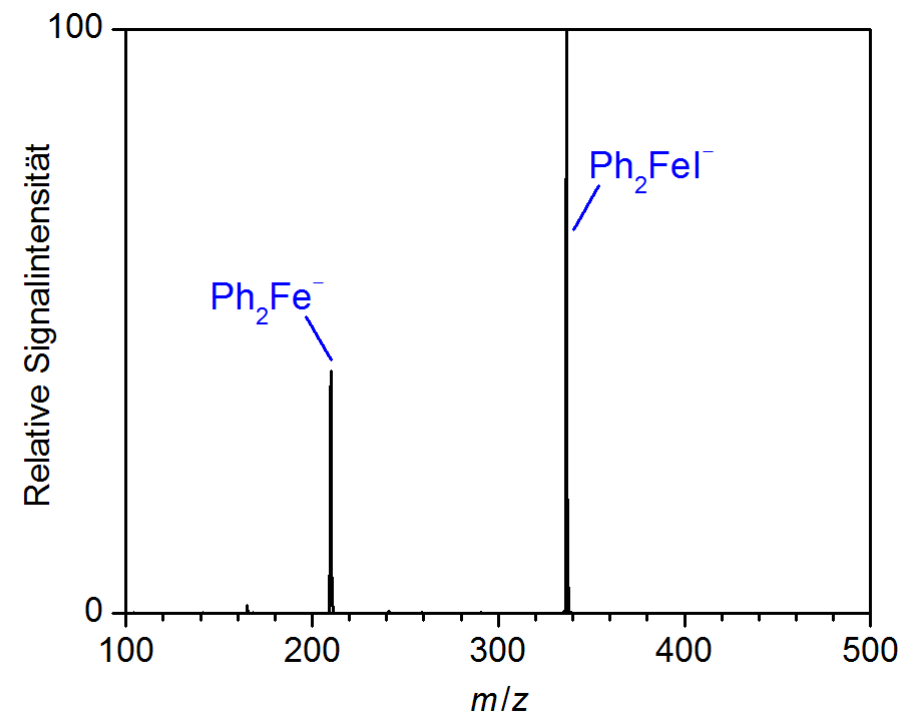

Abbildung 62. Massenspektrum von Massen-selektiertem $\mathrm{Ph}_{2} \mathrm{Fe}^{-}(\mathrm{m} / \mathrm{z} 210)$ und seiner Produkt-lonen nach Reaktion mit ${ }^{t}$ Bul.

Eine analoge Reaktivität ließ sich für die niedervalenten Spezies $\mathrm{PhFe}(0)\left(\mathrm{PPh}_{3}\right)^{-}$und $\mathrm{Fe}(-\mathrm{I})\left(\mathrm{PPh}_{3}\right)^{-}$durch Bildung von $\mathrm{PhFe}(\mathrm{I}) \mathrm{I}\left(\mathrm{PPh}_{3}\right)^{-}$bzw. Fe(0)I( $\left(\mathrm{PPh}_{3}\right)^{-}\left(\rightarrow \mathrm{Schema}^{20}\right)$ beobachten. Festzuhalten blieb zudem, dass gegenüber lodbenzol als aromatischem Reaktionspartner neben $\mathrm{Ph}_{2} \mathrm{Fe}(\mathrm{I})^{-}$nur noch die Eisen(0)-Spezies reaktiv war.
(a) $\mathrm{PhFe}\left(\mathrm{PPh}_{3}\right)^{-}+\mathrm{R}^{\prime} \mathrm{I}$
$\mathrm{PhFel}\left(\mathrm{PPh}_{3}\right)^{-}+\mathrm{R}^{\prime}$
(b) $\quad \mathrm{Fe}\left(\mathrm{PPh}_{3}\right)^{-}+\mathrm{R}^{\prime} \mathrm{I}$
$\operatorname{Fel}\left(\mathrm{PPh}_{3}\right)^{-}+\mathrm{R}^{\prime}$

Schema 20. (a) Bildung von PhFel( $\left.\mathrm{PPh}_{3}\right)^{-}$unter Freisetzung eines Organylradikals durch Reaktion der Eisen(0)Spezies PhFe( $\left.\mathrm{PPh}_{3}\right)^{-}$mit Organyliodiden R'I. (b) Bildung von Fel( $\left.\mathrm{PPh}_{3}\right)^{-}$unter Freisetzung eines Organlyradikals durch Reaktion der Eisen(-I)-Spezies Fe(PPh$)^{-}$mit Organyliodiden R'I.

Der Befund, dass Organoferrate in der Gasphase nur mit Organyliodiden reagierten, spiegelt die geringe Bindungsdissoziationsenergie der C-I-Bindung im Vergleich mit anderen Kohlenstoff-Halogen-Bindungen wider ( $\rightarrow$ Tabelle 12).$^{\left[{ }^{[92}\right]}$ 
Tabelle 12. Mittlere Bindungsdissoziationsenergien $D_{298}^{0} / \mathrm{kJ} \mathrm{mol}^{-1}$ von Kohlenstoff-Halogen-Bindungen C-Hal. ${ }^{[92]}$

\begin{tabular}{lc}
\hline $\mathbf{C}-\mathrm{Hal}$ & $\boldsymbol{D}_{\mathbf{2 9 8}}^{\mathbf{0}} / \mathbf{~ k J ~ m o l}^{-1}$ \\
\hline $\mathrm{C}-\mathrm{F}$ & $513.8 \pm 10.0$ \\
$\mathrm{C}-\mathrm{Cl}$ & $394.9 \pm 13.4$ \\
$\mathrm{C}-\mathrm{Br}$ & $318.0 \pm 8.4$ \\
$\mathrm{C}-\mathrm{I}$ & $253.1 \pm 35.6$ \\
\hline
\end{tabular}

Um die spezifische Reaktivität von $\mathrm{Ph}_{2} \mathrm{Fe}(\mathrm{I})^{-}$gegenüber Etl, ${ }^{\text {P }}$ rl und ${ }^{\text {}} \mathrm{Bul}$ genauer zu beschreiben, wurden in diesen Fällen zusätzlich die entsprechenden bimolekularen Reaktionsgeschwindigkeitskonstanten $k_{2}$ bestimmt. So konnte verdeutlicht werden, dass die Natur des Kohlenstoffzentrums (primär vs. sekundär vs. tertiär), das das lodid bindet, keinen großen Einfluss auf die Bildung von $\mathrm{Ph}_{2} \mathrm{Fel}^{-}$hat ( $\rightarrow$ Tabelle 13). Für $\mathrm{R}=\mathrm{Et}$ und $\mathrm{R}={ }^{t} \mathrm{Bu}$ konnten zusätzlich Reaktionseffizienzen von $\varphi=29 \%$ und $\varphi=23 \%$ ermittelt werden.

Tabelle 13. Bimolekulare Reaktionsgeschwindigkeitskonstanten $k_{2}$ für die Reaktion von $\mathrm{Ph}_{2} \mathrm{Fe}(\mathrm{I})^{-}$mit $\mathrm{R}^{\prime} \mathrm{I}$ mit den entsprechenden Reaktionseffizienzen $\varphi^{a}$ sowie den Bindungsdissoziationsenergien $D_{298}^{0}$ der Substrate. ${ }^{[92]}$

\begin{tabular}{lccc}
\hline $\mathbf{R}^{\prime}=$ & $\boldsymbol{k}_{\mathbf{2}} / \mathbf{c m}^{\mathbf{3}} \mathbf{s}^{\mathbf{- 1}}$ & $\boldsymbol{\varphi} / \boldsymbol{\%}$ & $\boldsymbol{D}_{\mathbf{2 9 8}}^{\mathbf{0}} / \mathbf{k J ~ m o l}^{-\mathbf{1}}$ \\
\hline $\mathrm{Et}$ & $(3.42 \pm 0.23) \cdot 10^{-10}$ & 29 & $233.5 \pm 6.3$ \\
${ }^{\mathrm{Pr}}$ & $(3.08 \pm 0.20) \cdot 10^{-10}$ & - & $234.7 \pm 6.3$ \\
${ }^{t} \mathrm{Bu}$ & $(3.22 \pm 0.23) \cdot 10^{-10}$ & 23 & $227.2 \pm 6.3$ \\
\hline
\end{tabular}

${ }^{a} \varphi=k_{2} / k_{\text {Stoß }}$; die Stoßrate $k$ Stoß wurde nach der Capture-Theorie berechnet. ${ }^{[93]}$ Diese konnte für $\mathrm{R}^{\prime}=$ 'Pr aufgrund des fehlenden Literaturwertes für die Polarisierbarkeit $\alpha$ nicht angewandt werden. Auch für $\mathrm{R}={ }^{t} \mathrm{Bu}$ fehlt ein Literaturwert für $\alpha$. Zur Berechnung der Stoßrate wurde hier angenommen, dass $\alpha\left({ }^{\mathrm{t} B u l}\right) \approx \alpha\left({ }^{\mathrm{n}} \mathrm{Bul}\right)$ gilt, da die Literaturwerte für $\alpha$ der entsprechenden Organylchloride annähernd identisch sind. ${ }^{\left[{ }^{[9 c]}\right.}$

In Vorexperimenten konnten erste Hinweise dafür gefunden werden, dass die Phenylferrate mit Eisen(0)- und Eisen(-I)-Zentren in Relation zu $\mathrm{Ph}_{2} \mathrm{Fe}(\mathrm{I})^{-}$langsamer mit Organyliodiden reagieren. Auch zeigten erste Vorversuche, dass die Reaktion der Phenylferrate mit aliphatischen Halogeniden unabhängig von der Oxidationsstufe der Eisen-Zentren schneller ablief als die entsprechende Reaktion mit Phenyliodid. Diese Tatsache steht im Einklang mit der allgemein geringeren Bindungsdissoziationsenergie von Alk-I gegenüber Ph-I. ${ }^{\left[{ }^{92,94]}\right.} \mathrm{Im}$ Gegensatz dazu verliefen die Reaktionen von $\mathrm{Ph}_{2} \mathrm{Fe}(\mathrm{I})^{-}$mit $\mathrm{Etl}$, ${ }^{i} \mathrm{Prl}$ und ${ }^{\mathrm{t}} \mathrm{Bul}$ in vergleichbarer Geschwindigkeit ab. Dies ist nicht überraschend, da sich die entsprechenden Bindungsdissoziationsenergien der aliphatischen Substrate innerhalb der Fehlergrenzen nicht unterscheiden ( $\rightarrow$ Tabelle 13). 
Die Halogen-Abstraktion an sich kann als der erste Schritt einer schrittweise ablaufenden oxidativen Addition betrachtet werden ( $\rightarrow$ Schema 21). Dass diese Reaktion selbst mit Phenyliodid als Reaktionspartner in der Gasphase beobachtet werden konnte, spiegelt die hohe Reaktivität der Eisen(I)-Spezies wider, da in diesem speziellen Fall durch homolytische Bindungsspaltung der C-I-Bindung ein energetisch ungünstiges Phenyl-Radikal erzeugt wird. Der zweite Schritt dieses Additionsprozesses konnte in der Gasphase nicht direkt beobachtet werden. Hierfür müsste nach Erzeugung des Produktions $\mathrm{Ph}_{2} \mathrm{Fel}^{-}$ein weiterer Stoß mit freigesetzten Ph-Radikalen erfolgen. Dieser Stoßprozess ist als sehr unwahrscheinlich anzunehmen. Hingegen können die entstehenden Radikale in der kondensierten Phase im Solvenskäfig verweilen, was ihre anschließende Rekombination erleichtert.

$$
\mathrm{Ph}_{2} \mathrm{Fe}^{-}+\mathrm{R}^{\prime} \mathrm{l} \longrightarrow \mathrm{Ph}_{2} \mathrm{Fel}^{-}+\mathrm{R}^{\prime} \longrightarrow \mathrm{Ph}_{2} \mathrm{FeR}^{\prime}{ }^{-}
$$

Schema 21. Schrittweise ablaufende oxidative Addition eines Organyliodids an $\mathrm{Ph}_{2} \mathrm{Fe}(\mathrm{I})^{-}$. 


\subsection{Reduktive Eliminierung und Konkurrenzreaktionen}

Stoßinduzierte Dissoziationsexperimente von Organoferraten in einer dreidimensionalen Quadrupol-Ionenfalle bieten eine ideale Möglichkeit, um deren intrinsische Reaktivität auf mikroskopischer Ebene in einem solvensfreien Raum zu untersuchen. Die dabei auftretenden Fragmentierungskanäle und Einflüsse auf diese wurden im Detail untersucht und lieferten wertvolle Informationen über die Freisetzung des Kreuzkupplungsproduktes als den entscheidenden Reaktionsschritt Eisen-katalysierter Kreuzkupplungsreaktionen.

\subsubsection{Reduktive Eliminierung}

Für die unimolekulare Reaktivität der Phenylferrate spielte die reduktive Eliminierung eine entscheidende Rolle. So eliminierte die Eisen(III)-Spezies $\mathrm{Ph}_{4} \mathrm{Fe}^{-}$mit $\mathrm{Ph}_{2}$ das Homokupplungsprodukt ( $\rightarrow$ Abbildung 63, Schema 22). Ein schrittweiser Verlust von PhenylRadikalen kann ausgeschlossen werden, da die Zwischenstufe $\mathrm{Ph}_{3} \mathrm{Fe}^{-}$ein anderes Fragmentierungsverhalten zeigte (siehe Kapitel 4.3.4). Auch für die polynuklearen Phenylferrate $\mathrm{Ph}_{5} \mathrm{Fe}_{2}{ }^{-}, \mathrm{Ph}_{7} \mathrm{Fe}_{4}{ }^{-}$und $\mathrm{Ph}_{8} \mathrm{Fe}_{5}{ }^{-}$war eine analoge Reaktivität zu beobachten.

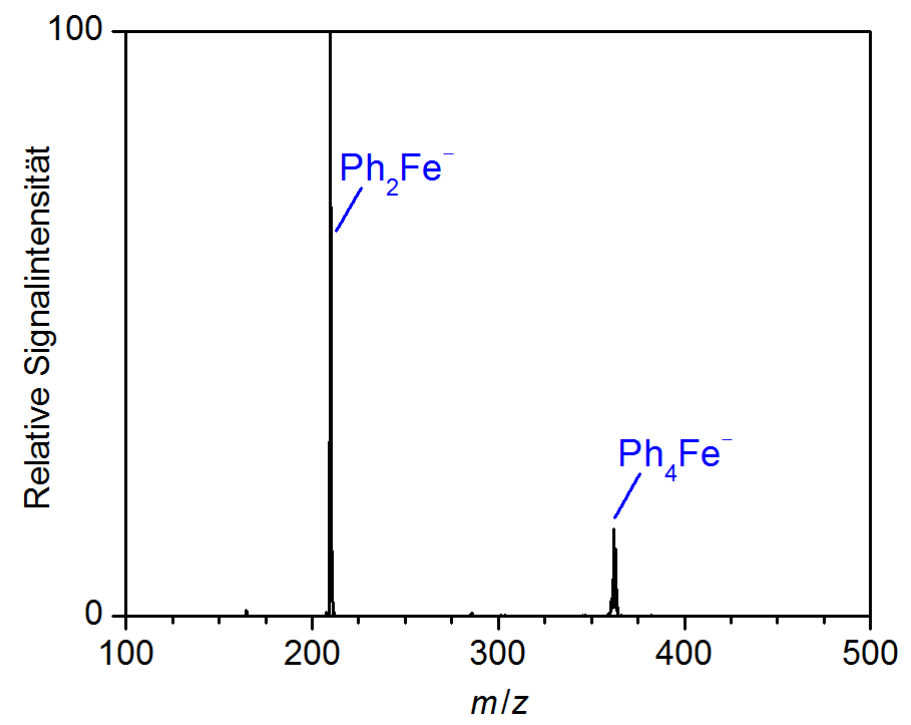

Abbildung 63. Massenspektrum von $\mathrm{Ph}_{4} \mathrm{Fe}^{-}(\mathrm{m} / \mathrm{z}$ 364) nach Isolation und anschließender Fragmentierung über stoßinduzierte Dissoziation $\left(V_{\text {Anr }}=0.6 \mathrm{~V}\right)$.

$$
\mathrm{Ph}_{4} \mathrm{Fe}^{-} \longrightarrow \mathrm{Ph}_{2} \mathrm{Fe}^{-}+\mathrm{Ph}-\mathrm{Ph}
$$

Schema 22. Unimolekulare Gasphasenreaktivität von $\mathrm{Ph}_{4} \mathrm{Fe}^{-}$über reduktive Eliminierung des Homokupplungsproduktes. 
Trugen die Phenylferrate monodentate $\left(\mathrm{PPh}_{3}\right)$ oder bidentate (dppbz) Phosphanliganden, war es durch stoßinduzierte Fragmentierungsexperimente wiederum möglich, das Homokupplungsprodukt freizusetzen. Auch die Komplexe $\mathrm{Fe}(\mathrm{dppbz})^{-}$und $\mathrm{PhFe}(\mathrm{dppbz})^{-}$zeigten einen verwandten Fragmentierungskanal, wobei die Phenylreste aus Fragmentierung der Liganden resultierten.

Die reduktive Eliminierung des Homokupplungsproduktes war nicht nur auf den Zerfall homoleptischer Phenylferrate beschränkt. So zeigten mit $\left(\mathrm{Ar}_{\mathrm{F}}\right)_{4} \mathrm{Fe}^{-}$und $(p-\mathrm{Tol})_{4} \mathrm{Fe}^{-}$weitere arylische Eisen(III)-Spezies dieses Fragmentierungsverhalten, wohingegen die analogen Organoferrate mit Eisen in der Oxidationsstufe + Il anderweitig fragmentierten (siehe Kapitel 4.3.4). Für homoleptische Alkylferrate spielte die reduktive Eliminierung eine eher untergeordnete Rolle und konnte für den Zerfall von $\mathrm{R}_{4} \mathrm{Fe}(\mathrm{III})^{-}(\mathrm{R}=\mathrm{Bu}$, Hex, Oct, Dec) nur in Spuren nachgewiesen werden (siehe Kapitel 4.3.2).

Bei Gasphasen-Fragmentierungsexperimenten heteroleptischer Komplexe des Typs $\mathrm{Ph}_{3} \mathrm{Fe}(\mathrm{III}) \mathrm{R}^{\prime-}$ konkurrierte die reduktive Eliminierung des Homokupplungsproduktes mit der des Kreuzkupplungsproduktes ( $\rightarrow$ Schema 23 ).

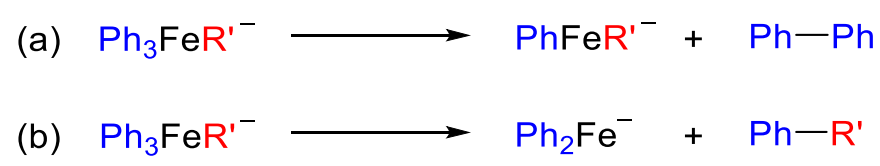

Schema 23. Unimolekulare Gasphasenreaktivität von $\mathrm{Ph}_{3} \mathrm{FeR}^{\prime-}$ über reduktive Eliminierung des Homokupplungsproduktes (a) oder des Kreuzkupplungsproduktes (b).

Für heteroleptische Komplexe $\mathrm{Ph}_{3} \mathrm{Fe}(\mathrm{III}) \mathrm{R}^{\prime-}$ mit Alkylsubstituenten (R' = Me, Et, 'Pr, Bu, Dec) resultierte die stoßinduzierte Fragmentierung der heteroleptischen Komplexe $\mathrm{Ph}_{3} \mathrm{Fe}(\mathrm{III}) \mathrm{R}^{\prime-}$ in einer hochselektiven Freisetzung des Kreuzkupplungsproduktes $\mathrm{Ph}-\mathrm{R}^{\prime}$. Repräsentativ für diese Experimente soll das Fragmentierungsexperiment von $\mathrm{Ph}_{3} \mathrm{Fe}^{i} \mathrm{Pr}^{-}$stehen, in dem die reduktive Eliminierung ausschließlich zur Bildung des Kreuzkupplungsproduktes führte $\left(\rightarrow\right.$ Abbildung 64). Zusätzlich waren Spuren an $\mathrm{PhO}^{-}$und $\left[\mathrm{Ph}, \mathrm{Fe}, \mathrm{O}_{2}\right]^{-} \mathrm{zu}$ erkennen. Diese Anionen deuteten auf eine bimolekulare Reaktion mit Sauerstoff hin, der in geringen Mengen im Vakuumsystem des Massenspektrometers vorhanden war. Auch die alkylreicheren Spezies $\mathrm{Ph}_{2} \mathrm{FeDec}_{2}{ }^{-}$und $\mathrm{PhFeR}_{3}{ }^{-}\left(\mathrm{R}^{\prime}=\mathrm{Bu}, \mathrm{Dec}\right)$ zeigten dieselbe Tendenz, doch waren im Falle der letztgenannten Spezies weitere Fragmentierungskanäle offensichtlich (siehe Kapitel 4.3.2 und 4.3.4). Hingegen konnte für $\mathrm{Ph}_{3} \mathrm{FeBn}^{-}$und $\mathrm{Ph}_{2} \mathrm{FeBn}_{2}{ }^{-}$der Zerfall über Bildung der Homokupplungsprodukte $\mathrm{Ph}_{2}$ bzw. $\mathrm{Bn}_{2}$ in der Gasphase beobachtet werden. 


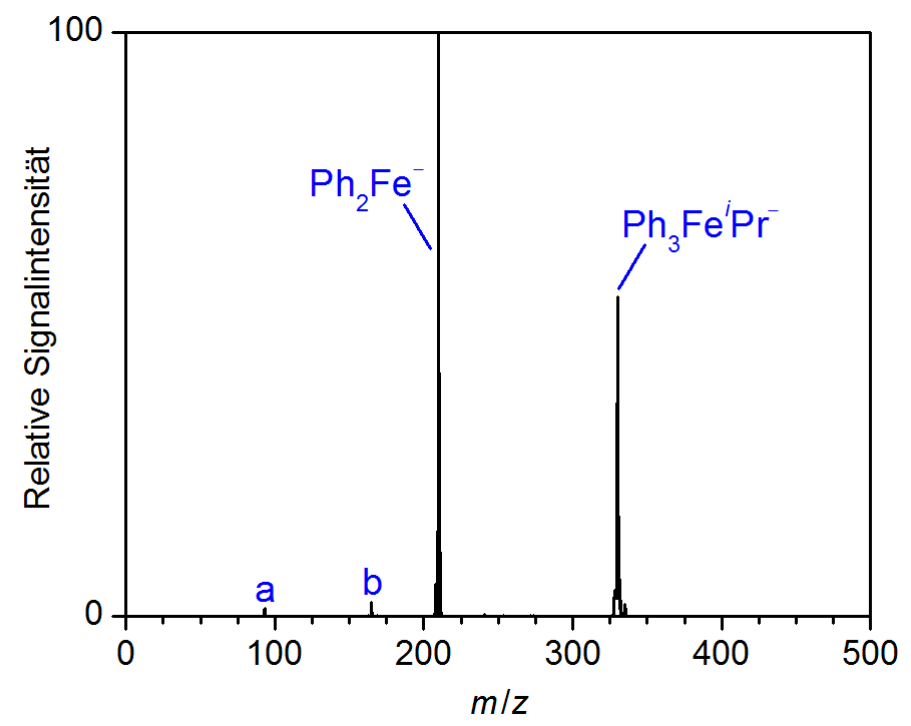

Abbildung 64. Massenspektrum von $\mathrm{Ph}_{3} \mathrm{Fe}^{i} \mathrm{Pr}^{-}(\mathrm{m} / \mathrm{z} 330)$ nach Isolation und anschließender Fragmentierung über stoßinduzierte Dissoziation $\left(\mathrm{V}_{\mathrm{Anr}}=0.3 \mathrm{~V}\right) ; \mathrm{a}=\mathrm{PhO}^{-}, \mathrm{b}=\left[\mathrm{Ph}, \mathrm{Fe}, \mathrm{O}_{2}\right]^{-}$.

Für heteroleptische Komplexe $\mathrm{Ph}_{3} \mathrm{FeR}^{-}$, die neben den Phenyl-Substituenten eine weitere aromatische Gruppe aufwiesen, wurden elektronische Einflüsse auf die reduktive Eliminierung im Detail untersucht. Hierzu wurden aromatische Reste mit verschiedenen para-Substituenten in die heteroleptischen Anionen eingebracht und die Auswirkungen auf deren unimolekulare Gasphasenreaktivität untersucht. Für $\quad \mathrm{R}^{\prime}=p-\mathrm{NMe}_{2}-\mathrm{C}_{6} \mathrm{H}_{4} \quad$ mit einer stark elektronendonierenden $\mathrm{NMe}_{2}$-Gruppe zeigte sich im entsprechenden GasphasenFragmentierungsexperiment eine starke Präferenz der Kreuzkupplung über die Homokupplung ( $\rightarrow$ Abbildung 65).

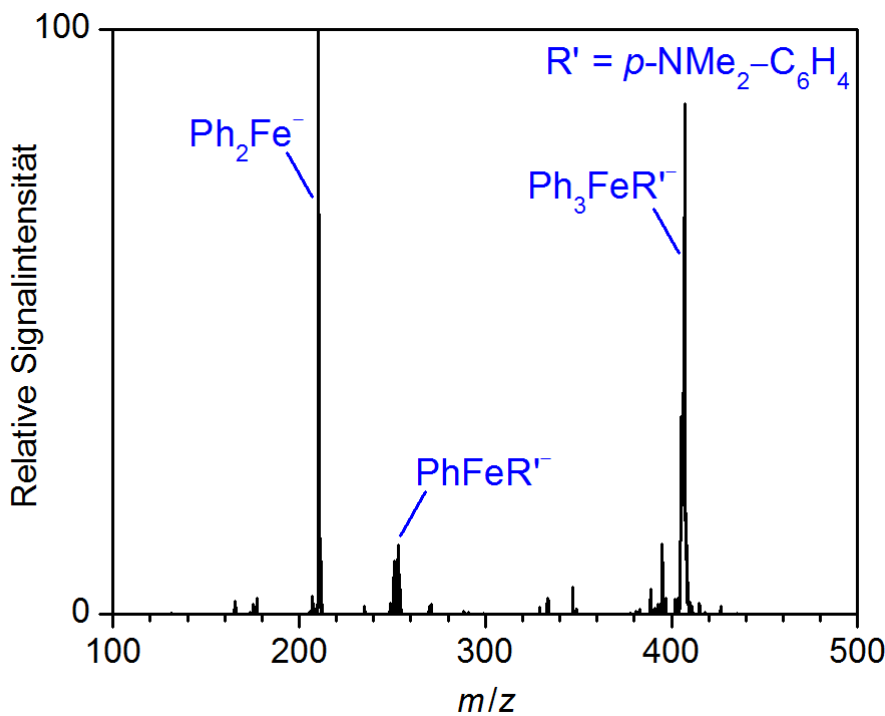

Abbildung 65. Massenspektrum von $\mathrm{Ph}_{3} \mathrm{FeR}^{-}(\mathrm{m} / \mathrm{z}$ 407) nach Isolation und anschließender Fragmentierung über stoßinduzierte Dissoziation $\left(V_{\text {Anr }}=0.9 \mathrm{~V}\right) ; \mathrm{R}^{\prime}=p-\mathrm{NMe}_{2}-\mathrm{C}_{6} \mathrm{H}_{4}$. 
Für die heteroleptischen Anionen mit weniger elektronenreichen Aryl-Substituenten $\left(\mathrm{R}^{\prime}=p-\mathrm{OMe}-\mathrm{C}_{6} \mathrm{H}_{4}, p-\mathrm{Me}-\mathrm{C}_{6} \mathrm{H}_{4}, p-\mathrm{Ph}-\mathrm{C}_{6} \mathrm{H}_{4}\right)$ nahm die Homokupplung an Bedeutung zu. Wenn die heteroleptischen Anionen mit $\mathrm{R}^{\prime}=p-\mathrm{F}-\mathrm{C}_{6} \mathrm{H}_{4}$ oder $\mathrm{R}^{\prime}=p-\mathrm{OCHF}_{2}-\mathrm{C}_{6} \mathrm{H}_{4}$ einen elektronenarmen Aromaten enthielten, konnte eine Dominanz der Homokupplung über die Kreuzkupplung in den entsprechenden Gasphasen-Fragmentierungsexperimenten beobachtet werden. Mit Chlorid als einem vergleichsweise stark elektronenziehenden Substituenten ließ sich für die stoßinduzierte Dissoziation der heteroleptischen Eisen(III)Spezies $\mathrm{Ph}_{3} \mathrm{FeR}^{\prime-}\left(\mathrm{R}^{\prime}=p-\mathrm{Cl}-\mathrm{C}_{6} \mathrm{H}_{4}\right)$ nur noch der Homokupplungskanal nachweisen $(\rightarrow$ Abbildung 66). Die entsprechenden heteroleptischen Anionen mit stark elektronenarmen Aryl-Substituenten $\left(\mathrm{R}^{\prime}=p-\mathrm{CN}-\mathrm{C}_{6} \mathrm{H}_{4}, p-\mathrm{CF}_{3}-\mathrm{C}_{6} \mathrm{H}_{4}\right)$ waren nicht zugänglich und konnten somit im Rahmen dieser Experimente nicht berücksichtigt werden.

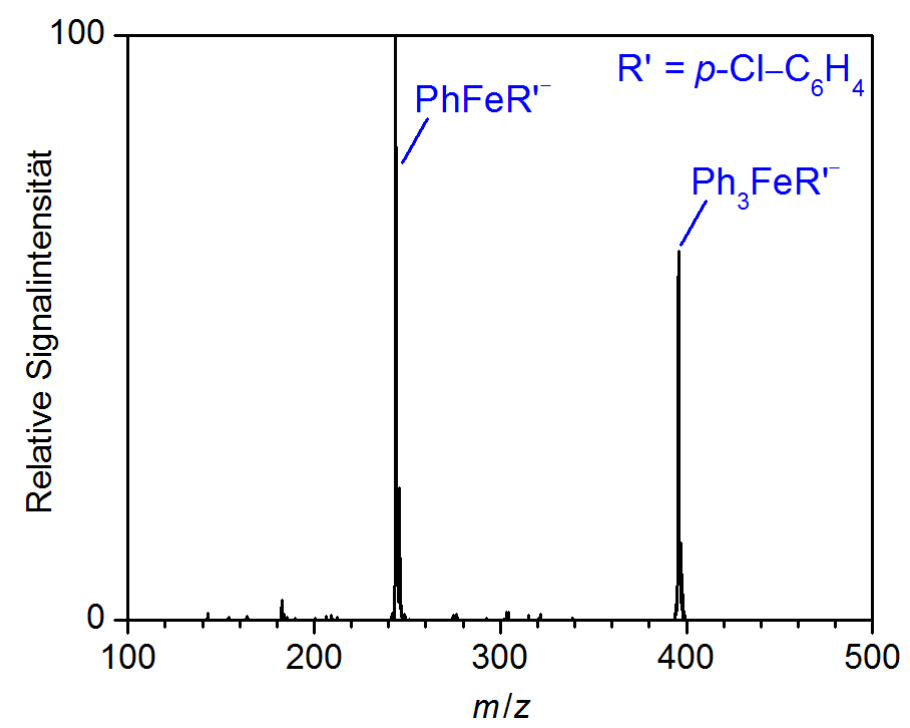

Abbildung 66. Massenspektrum von $\mathrm{Ph}_{3} \mathrm{FeR}^{\prime-}(\mathrm{m} / \mathrm{z}$ 398) nach Isolation und anschließender Fragmentierung über stoßinduzierte Dissoziation $\left(V_{\mathrm{Anr}}=0.8 \mathrm{~V}\right) ; \mathrm{R}^{\prime}=p-\mathrm{Cl}-\mathrm{C}_{6} \mathrm{H}_{4}$.

Die Signalintensität der Fragmentionen $\mathrm{Ph}_{2} \mathrm{Fe}^{-}$und $\mathrm{PhFeR}^{-}$und das Verhältnis dieser Anionen zueinander waren abhängig von der Anregungsspannung $V_{\text {Anr, }}$ die im Fragmentierungsexperiment angewendet wurde. Eine Auftragung der Signalintensitäten I der Fragmentionen normiert auf die Signalintensität $l_{0}$ von $\mathrm{Ph}_{3} \mathrm{FeR}^{-}$(bei $V_{\text {Anr }}=0 \mathrm{~V}$ ) gegen die Anregungsspannung $V_{\text {Anr }}$ zeigte einen sigmoidalen Verlauf der Datenpunkte. Dies soll an einer Auftragung der Zerfallskanäle für Homokupplung und Kreuzkupplung ausgehend von $\mathrm{Ph}_{3} \mathrm{Fe}(p-\mathrm{Tol})^{-}$verdeutlicht werden, aus der hervorgeht, dass der Kreuzkupplungskanal leicht bevorzugt auftrat ( $\rightarrow$ Abbildung 67). Aus Auftragungen dieser Art konnten die Auftrittsspannung aller Zerfallskanäle in Abhängigkeit von der Arylgruppe und deren para-Substituent 
direkt bestimmt werden ( $\rightarrow$ Tabelle 14). Diese sind im Allgemeinen eng verwandt mit der Energiebarriere der entsprechenden Gasphasenreaktionen.

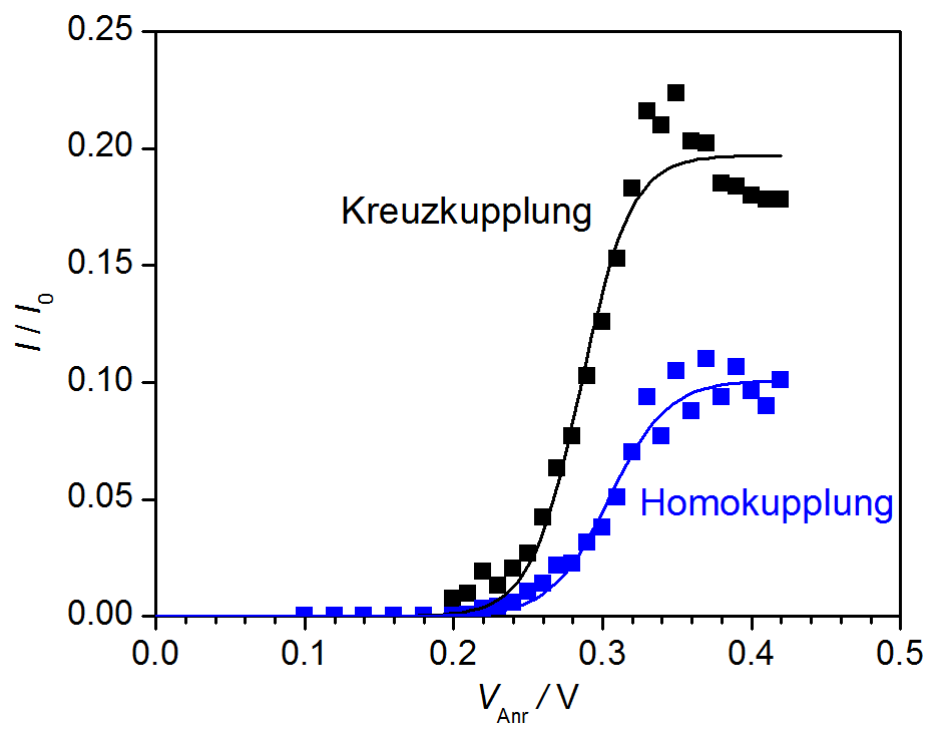

Abbildung 67. Auftragung und sigmoidale Anpassungen der normierten Signalintensitäten $/ /$ lo der Fragmentionen, die durch stoßinduzierte Dissoziation von massenselektierten $\mathrm{Ph}_{3} \mathrm{Fe}(p-\mathrm{Tol})^{-}$in der Gasphase erzeugt werden konnten, gegen die Anregungsspannungen $V_{\text {Anr }}\left(l_{0}=\right.$ Signalintensität von $\mathrm{Ph} 3 \mathrm{Fe}(p-\mathrm{Tol})^{-}$für $V_{\mathrm{Anr}}=0$.).

Tabelle 14. Bevorzugte Zerfallskanäle und Auftrittsspannung beider Zerfallskanäle, die in Abhängigkeit vom Substituenten $X$ aus den Gasphasen-Fragmentierungsexperimenten der Arylferrate $\mathrm{Ph}_{3} \mathrm{Fe}\left(p-\mathrm{X}-\mathrm{C}_{6} \mathrm{H}_{4}\right)^{-}$ermittelt werden konnten.

\begin{tabular}{lccc}
\hline $\mathbf{X}=$ & $\begin{array}{c}\text { Bevorzugter } \\
\text { Zerfallskanal }\end{array}$ & $\begin{array}{c}\text { Auftrittsspannung } \\
\text { Kreuzkupplung / V }\end{array}$ & $\begin{array}{c}\text { Auftrittsspannung } \\
\text { Homokupplung / V }\end{array}$ \\
\hline $\mathrm{NMe}_{2}$ & Kreuzkupplung & - & - \\
$\mathrm{OMe}$ & Kreuzkupplung & $0.12 \pm 0.01$ & $0.12 \pm 0.01$ \\
$\mathrm{Me}$ & Kreuzkupplung & $0.25 \pm 0.01$ & $0.26 \pm 0.01$ \\
$\mathrm{Ph}$ & Kreuzkupplung & $0.49 \pm 0.01$ & $0.54 \pm 0.01$ \\
$\mathrm{H}$ & Homokupplung & - & $0.23 \pm 0.02$ \\
$\mathrm{~F}$ & Homokupplung & $0.36 \pm 0.02$ & $0.39 \pm 0.03$ \\
$\mathrm{OCHF}_{2}$ & Homokupplung & $0.68 \pm 0.06$ & $0.68 \pm 0.06$ \\
$\mathrm{Cl}$ & Homokupplung & - & $0.43 \pm 0.03$ \\
\hline
\end{tabular}

Um die elektronischen Effekte auf die reduktive Eliminierung detaillierter zu analysieren, bot sich die Erstellung eines Hammett-Plots an. Da die Auftrittsspannungen in einer komplizierten und nicht eindeutigen Weise von dem $\mathrm{m} / \mathrm{z}$-Verhältnis der Fragmentionen abhängen und keine klaren Tendenzen offensichtlich waren, konnten diese nicht als Grundlage für den HammettPlot fungieren. Alternativ wurde das relative Verhältnis der Fragmentionen zueinander im Bereich der Anregungsspannungen genutzt, bei denen $\mathrm{Ph}_{3} \mathrm{FeR}^{\prime-}$ nahezu komplett dissoziiert 
vorlag ( $\rightarrow$ Tabelle 15). Zur Erstellung des Hammett-Plots wurden diese gegen die HammettParameter $\sigma_{\mathrm{p}}$ aufgetragen ( $\rightarrow$ Abbildung 68 ).

Tabelle 15. Hammett-Parameter $\sigma_{p}$ der Substituenten $X^{[95]}$ und das relative Verhältnis der normierten Signalintensitäten I der Zerfallskanäle ${ }^{a}$ ermittelt aus den Gasphasen-Fragmentierungsexperimenten der Arylferrate $\mathrm{Ph}_{3} \mathrm{Fe}\left(p-\mathrm{X}-\mathrm{C}_{6} \mathrm{H}_{4}\right)^{-}$.

\begin{tabular}{lcc}
\hline $\mathbf{X}=$ & $\begin{array}{c}\text { Hammett- } \\
\text { parameter } \boldsymbol{\sigma}_{\mathrm{p}}\end{array}$ & $\begin{array}{c}\boldsymbol{I} \text { (Kreuzkupplung) } / \\
\boldsymbol{I} \text { (Homokupplung) }\end{array}$ \\
\hline $\mathrm{NMe}_{2}$ & -0.83 & 10 \\
$\mathrm{OMe}$ & -0.27 & 1.4 \\
$\mathrm{Me}$ & -0.17 & 1.5 \\
$\mathrm{Ph}$ & -0.01 & 1.1 \\
$\mathrm{H}$ & 0.00 & 1.0 \\
$\mathrm{~F}$ & 0.06 & 0.086 \\
$\mathrm{OCHF}_{2}$ & 0.18 & $0.29^{b}$ \\
$\mathrm{Cl}$ & 0.23 & $<0.01^{c}$ \\
\hline
\end{tabular}

a Das relative Verhältnis der Signalintensitäten für die verschiedenen Fragmentionen wurde im Bereich der Anregungsspannungen bestimmt, in denen das ursprüngliche Anion $\mathrm{Ph} 3 \mathrm{Fe}\left(p-\mathrm{X}-\mathrm{C}_{6} \mathrm{H}_{4}\right)^{-}$nahezu vollständig dissoziiert vorlag. ${ }^{b}$ Für $\mathrm{X}=\mathrm{OCHF}_{2}$ konnten weitere Reaktionskanäle beobachtet werden, die auf den Zerfall des $p$-Substituenten zurückzuführen waren. ${ }^{c}$ Der Kreuzkupplungskanal konnte nicht beobachtet werden. Das angegebene Verhältnis ist als obere Grenze zu verstehen.

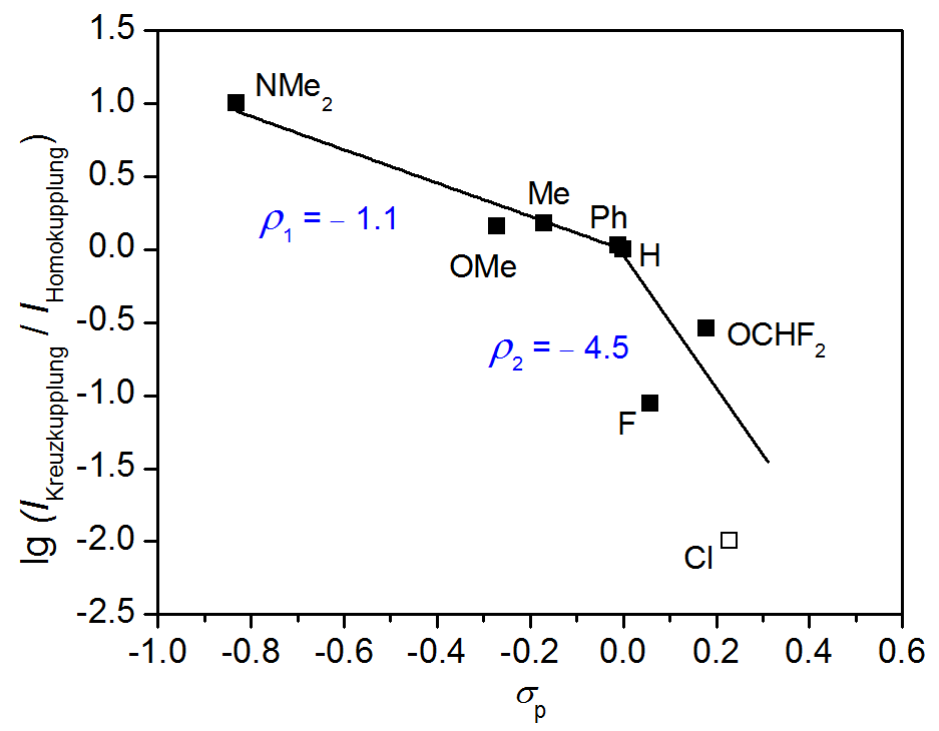

Abbildung 68. Hammett-Plot für das Verhältnis der Kreuz- und Homokupplungskanäle, die nach Isolation und anschließender Fragmentierung von $\mathrm{Ph}_{3} \mathrm{FeR}^{--}\left(\mathrm{R}^{\prime}=p-\mathrm{X}-\mathrm{C}_{6} \mathrm{H}_{4}\right)$ in der Gasphase beobachtet werden konnten. Für $X=\mathrm{NMe}_{2}$ bis $\mathrm{X}=\mathrm{H}$ sowie für $\mathrm{X}=\mathrm{H}$ bis $\mathrm{X}=\mathrm{OCHF}_{2}$ wurden die Datenpunkte mit den Steigungen $\rho_{1}$ und $\rho_{2}$ linear angepasst. Der Datenpunkt für $\mathrm{X}=\mathrm{Cl}$ entspricht einem oberen Grenzwert und wurde für die linearen Anpassungen nicht berücksichtigt, da in diesem Fall nur der Homokupplungskanal zu beobachten war. 
Der Hammett-Plot belegte, dass für elektronendonierende Substituenten die Kreuzkupplung klar bevorzugt wurde, wohingegen elektronenziehende Substituenten primär die reduktive Eliminierung des Homokupplungsproduktes bewirkten. Die Steigung des Hammett-Plots zeigte einen negativen Betrag, der aber nicht konstant war. So konnte für den Bereich elektronendonierender Substituenten eine Steigung von $\rho_{1}=-1.1$ ausgemacht werden. Hingegen zeigte sich in der Region elektronenziehender Substituenten mit $\rho_{2}=-4.5$ eine vom Betrag her größere Steigung.

Zusätzliche Informationen über die elektronischen Einflüsse auf die reduktive Eliminierung konnte durch stoßinduzierte Dissoziation von $\mathrm{Ph}_{n} \mathrm{FeR}_{4-n}{ }^{-}(n \leq 2)$ gewonnen werden. Einige dieser Anionen konnten für $\mathrm{R}^{\prime}=p-\mathrm{F}-\mathrm{C}_{6} \mathrm{H}_{4}$ und $\mathrm{R}^{\prime}=p-\mathrm{CF}_{3}-\mathrm{C}_{6} \mathrm{H}_{4}$ gebildet werden. Alle untersuchten Anionen des letztgenannten Typs zerfielen über reduktive Eliminierung in der Gasphase. So setzte $\mathrm{Ph}_{2} \mathrm{FeR}_{2}{ }^{-}$(zu beachten ist das mögliche Vorliegen einer Mischung aus cis- und trans-Isomeren im Falle einer quadratisch-planaren Struktur; $\mathrm{R}^{\prime}=p-\mathrm{F}-\mathrm{C}_{6} \mathrm{H}_{4}$ ) in der Gasphase primär $\mathrm{Ph}_{2}$ frei. Auch $\mathrm{PhFeR}_{3}{ }^{-}$zeigte ein analoges Verhalten in der Gasphase, wobei $\mathrm{Ph}_{2}$ in geringerer Selektivität gebildet wurde. Für $\mathrm{PhFeR}^{\prime}{ }^{-}\left(\mathrm{R}^{\prime}=p-\mathrm{CF}_{3}-\mathrm{C}_{6} \mathrm{H}_{4}\right)$ mit einem stärker elektronenziehenden Substituenten konnte ausschließlich die Bildung des Kreuzkupplungsproduktes Ph-R' nachgewiesen werden.

Auf eine ähnliche Art und Weise wurde der Einfluss sterischer Effekte auf die reduktive Eliminierung in der Gasphase im Detail untersucht. So wurden gezielt Arylsubstituenten R' mit einem hohen sterischen Anspruch in Anionen des Typs $\mathrm{Ph}_{3} \mathrm{FeR}^{--}$eingebracht. So zeigte sich in den durchgeführten Gasphasenexperimenten $\left(\mathrm{R}^{\prime}=\mathrm{o}-\mathrm{Tol}, \mathrm{o}-\mathrm{Ph}-\mathrm{C}_{6} \mathrm{H}_{4}, 0-\mathrm{CF}_{3}-\mathrm{C}_{6} \mathrm{H}_{4}\right)$ die stark bevorzugte oder ausschließlich auftretende Fragmentierung über reduktive Eliminierung des Homokupplungsproduktes $\mathrm{Ph}_{2}$. $\mathrm{Zu}$ beachten ist, dass aufgrund des elektronischen Einflusses des 0 -Substituenten für $\mathrm{R}^{\prime}=0-\mathrm{CF}_{3}-\mathrm{C}_{6} \mathrm{H}_{4}$ die Bevorzugung des Homokupplungskanals zu erwarten war (siehe oben). In den beiden anderen Fällen sollten die elektronischen Eigenschaften der o-Substituenten in die entgegengesetzte Richtung agieren, sodass hier von einem wesentlichen Einfluss sterischer Effekte auszugehen war. Um diese Tatsache zu untermauern, wurde $\mathrm{Ph}_{3} \mathrm{FeMes}^{-}$mit einer sterisch sehr anspruchsvollen Mesityl-Gruppe dem entsprechenden Gasphasen-Experiment unterzogen. Auch hier konnte ausschließlich die reduktive Eliminierung von $\mathrm{Ph}_{2}$ beobachtet werden ( $\rightarrow$ Abbildung 69). Phenylferrate mit Eisen in der Oxidationsstufe +IV zeigten kein einheitliches Fragmentierungsverhalten in der Gasphase. So ließ sich für $\mathrm{Ph}_{4} \mathrm{FeBu}^{-}$als Hauptzerfallskanal die reduktive Eliminierung des Homokupplungsproduktes $\mathrm{Ph}_{2}$ beobachten ( $\rightarrow$ Abbildung 70 und Schema 24). Weitere untersuchte Eisen(IV)-Spezies zeigten alternative Fragmentierungsmuster (siehe Kapitel 4.3.4). 


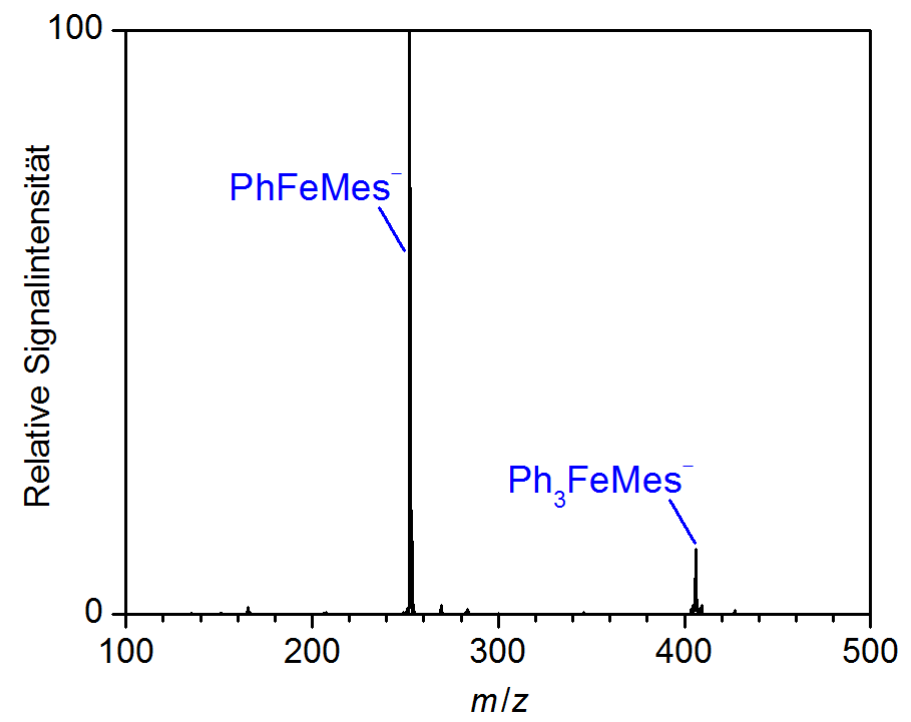

Abbildung 69. Massenspektrum von $\mathrm{Ph}_{3} \mathrm{FeMes}^{-}(\mathrm{m} / \mathrm{z}$ 406) nach Isolation und anschließender Fragmentierung über stoßinduzierte Dissoziation ( $\left.V_{\text {Anr }}=0.5 \mathrm{~V}\right)$.

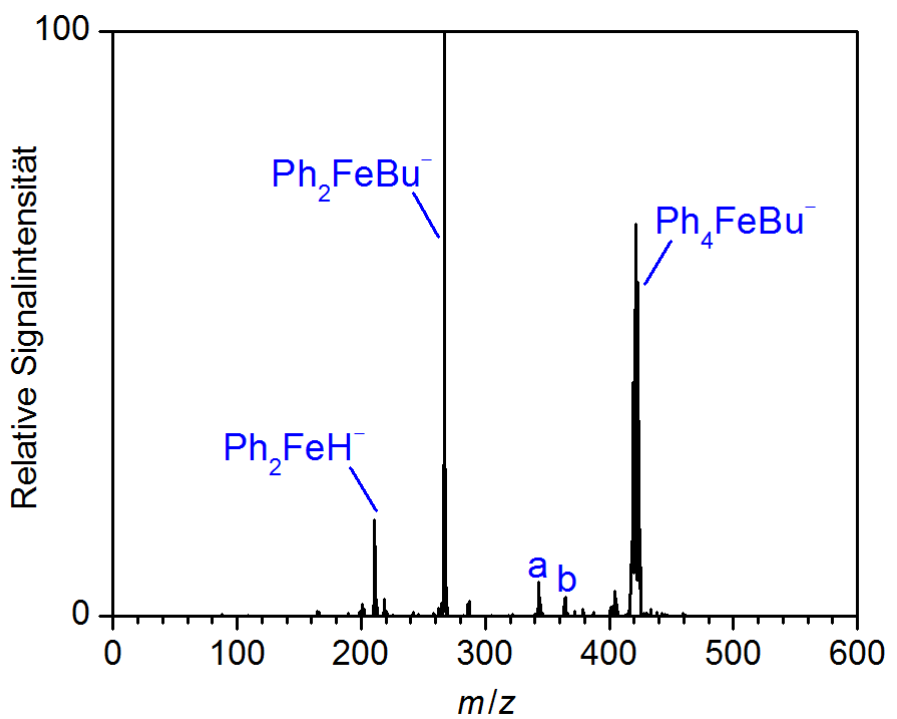

Abbildung 70. Massenspektrum von $\mathrm{Ph}_{4} \mathrm{FeBu}^{-}(\mathrm{m} / \mathrm{z}$ 421) nach Isolation und anschließender Fragmentierung über stoßinduzierte Dissoziation $\left(V_{\text {Anr }}=0.2 \mathrm{~V}\right) ; \mathrm{a}=\left[\mathrm{Ph}_{4} \mathrm{FeBu}-\mathrm{C}_{6} \mathrm{H}_{6}\right]^{-}, \mathrm{b}=\mathrm{Ph}_{4} \mathrm{Fe}^{-}+\mathrm{Ph}_{4} \mathrm{FeH}^{-}$.

$$
\mathrm{Ph}_{4} \mathrm{FeBu}^{-} \longrightarrow \mathrm{Ph}_{2} \mathrm{FeBu}^{-}+\mathrm{Ph}-\mathrm{Ph}
$$

Schema 24. Unimolekulare Gasphasenreaktivität von $\mathrm{Ph}_{4} \mathrm{FeBu}^{-}$mit reduktiver Eliminierung des Homokupplungsproduktes $\mathrm{Ph}_{2}$ als Hauptzerfallskanal. 
Für die gemischten Cluster-Anionen des Typs $\mathrm{Me}_{12-}{ }_{n} \mathrm{Fe}_{8} \mathrm{Ph}_{n}{ }^{-}(n=3-5)$ ließ sich bei dem Phenyl-reicheren Aggregaten der Zerfall über reduktive Eliminierung des Kreuzkupplungs- und des Homokupplungsproduktes beobachten, auch wenn in diesen speziellen Fällen weitere Fragmentierungskanäle präsent waren (siehe Kapitel 4.3.5). Auch für die heteroleptischen Organoferrate des Typs $\mathrm{Bu}_{3} \mathrm{FeR}^{--}\left(\mathrm{R}^{\prime}=\mathrm{Ph}, \quad p\right.$-Tol $)$ konnten mit der Freisetzung des Kreuzkupplungs- und des Homokupplungsproduktes reduktive Eliminierungen in der Gasphase nachgewiesen werden ( $\rightarrow$ Abbildung 71 und Schema 25). Speziell bei diesen Komplexen spielten weitere Zerfallskanäle eine dominante Rolle. So traten radikalische Prozesse (siehe Kapitel 4.3.2) und die Freisetzung von Neutralteilchen $\mathrm{RH}$ (siehe Kapitel 4.3.4) in den Vordergrund und sollen im weiteren Verlauf dieser Arbeit im Detail diskutiert werden.

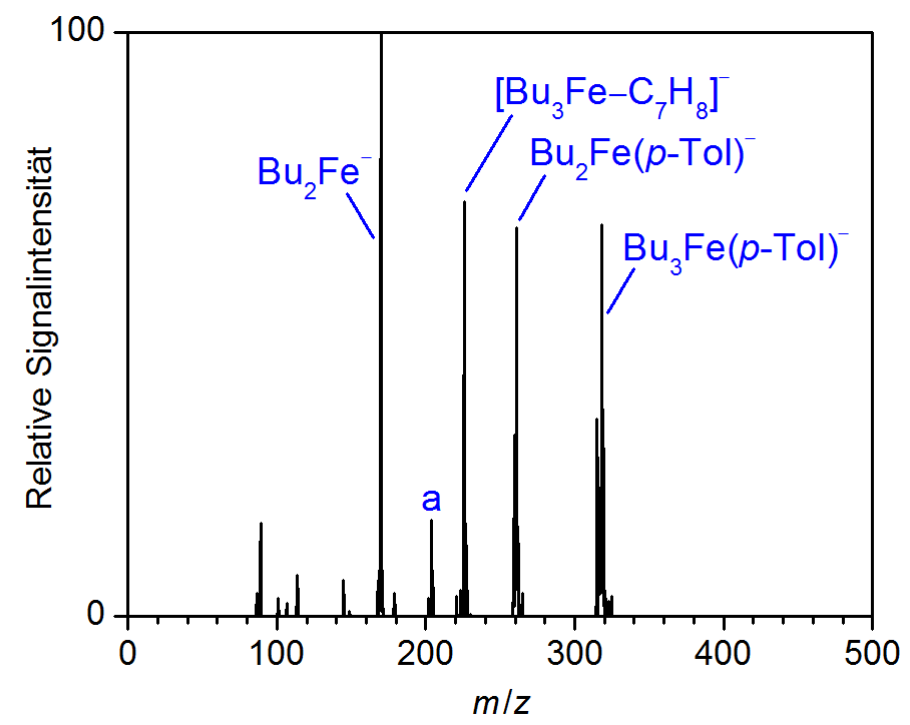

Abbildung 71. Massenspektrum von $\mathrm{Bu}_{3} \mathrm{FeR}^{-}(\mathrm{m} / \mathrm{z} 318)$ nach Isolation und anschließender Fragmentierung über stoßinduzierte Dissoziation $\left(V_{\text {Anr }}=0.35 \mathrm{~V}\right) ; \mathrm{a}=\operatorname{BuFe}(p-\mathrm{Tol})^{-}$.
(a) $\mathrm{Bu}_{3} \mathrm{FeR}^{-}$
$\mathrm{Bu}_{2} \mathrm{Fe}^{-}+\mathrm{Bu}-\mathrm{R}^{\prime}$
(b) $\mathrm{Bu}_{3} \mathrm{FeR}^{-}$
$\mathrm{BuFeR}^{-}+\mathrm{Bu}-\mathrm{Bu}$
(c) $\mathrm{Bu}_{3} \mathrm{FeR}^{\prime-}$
$\mathrm{Bu}_{2} \mathrm{FeR}^{\prime^{-}}+\mathrm{Bu}^{*}$
(d) $\mathrm{Bu}_{3} \mathrm{FeR}^{-}$
$\left[\mathrm{Bu}_{3} \mathrm{FeR}-\mathrm{R}^{\prime} \mathrm{H}\right]^{-}+\mathrm{R}^{\prime} \mathrm{H}$

Schema 25. Unimolekulare Gasphasenreaktivität von $\mathrm{Bu}_{3} F e \mathrm{R}^{-}\left(\mathrm{R}^{\prime}=\mathrm{Ph}, \mathrm{p}\right.$-Tol) mit reduktiver Eliminierung des Kreuzkupplungsproduktes (a) oder des Homokupplungsproduktes (b). In Konkurrenz zu diesen Reaktionen erfolgte die Freisetzung eines Butylradikals (c) oder eines Neutralteilchens R'H (d). 


\subsubsection{Freisetzung von Radikalen}

Neben der reduktiven Eliminierung spielten radikalische Prozesse beim Zerfall der Organoferrate eine wesentliche Rolle ( $\rightarrow$ Schema 26). So zerfielen $\mathrm{R}_{3} \mathrm{Fe}^{-}\left(\mathrm{R}=\mathrm{Bn}, \mathrm{Me}_{3} \mathrm{SiCH}_{2}\right)$ und $\mathrm{R}_{4} \mathrm{Fe}^{-}\left(\mathrm{R}=\mathrm{Me}, \mathrm{Bn}, \mathrm{Me}_{3} \mathrm{SiCH}_{2}\right)$ über Freisetzung der entsprechenden Organyl-Radikale R' ( $\rightarrow$ Abbildung 72). Auch für heteroleptische Eisen(II)- und Eisen(III)-Spezies sowie für höhere polynukleare Aggregate, die einen oder mehrere der zuletzt genannten organischen Reste aufwiesen, war dieser Zerfallskanal sehr prominent. Hingegen war die Freisetzung aromatischer Radikale eine Seltenheit und konnte nur für das Anion $\left(\operatorname{Ar}_{\mathrm{F}}\right)_{3} \mathrm{Fe}(\mathrm{II})^{-}$durch Eliminierung von $\left(\mathrm{Ar}_{\mathrm{F}}\right)^{\cdot}$ beobachtet werden $\left(\mathrm{Ar}_{\mathrm{F}}=3,5-\left(\mathrm{CF}_{3}\right)_{2}-\mathrm{C}_{6} \mathrm{H}_{3}\right)$.

$$
\mathrm{R}_{m} \mathrm{Fe}_{x}^{-} \longrightarrow \mathrm{R}_{m-1} \mathrm{Fe}_{x}^{-}+\mathrm{R}^{\cdot}
$$

Schema 26. Allgemeines Reaktionsschema für die unimolekulare Gasphasenreaktivität von $\mathrm{R}_{m} \mathrm{Fe}_{x}{ }^{-}$über Freisetzung eines Organyl-Radikals R:

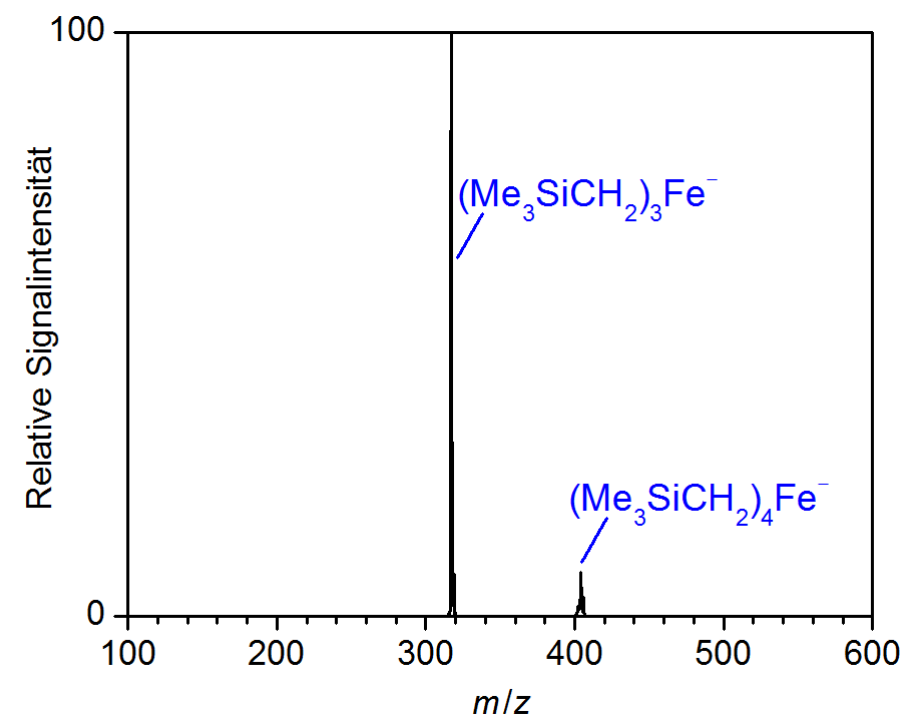

Abbildung 72. Massenspektrum von $\left(\mathrm{Me}_{3} \mathrm{SiCH}_{2}\right)_{4} \mathrm{Fe}^{-}(\mathrm{m} / \mathrm{z} 404)$ nach Isolation und anschließender Fragmentierung über stoßinduzierte Dissoziation ( $\left.V_{\text {Anr }}=0.40 \mathrm{~V}\right)$.

Die Fragmentierung über Abspaltung eines Radikals konnte auch für die Organoferrate mit längeren Alkylketten beobachtet werden, auch wenn in diesem Fall das Auftreten dieses Reaktionspfades stark von der Oxidationsstufe des Eisenkerns abhängig war. Für alle Organoferrate des Typs $\mathrm{R}_{4} \mathrm{Fe}(\mathrm{III})^{-}(\mathrm{R}=\mathrm{Bu}$, Hex, Oct, Dec) konnte die Eliminierung eines Alkylradikals direkt nachgewiesen werden, wohingegen die entsprechenden Eisen(II)-Spezies 
$\mathrm{R}_{3} \mathrm{Fe}(\mathrm{II})^{-}$über einen alternativen Zerfallskanal fragmentierten (siehe Kapitel 4.3.3). So setzte $\mathrm{Dec}_{4} \mathrm{Fe}(\mathrm{III})^{-}$in der Gasphase ein Decyl-Radikal frei ( $\rightarrow$ Abbildung 73 ), $\mathrm{Dec}_{3} \mathrm{Fe}(\mathrm{II})^{-}$jedoch nicht. Decyl-Radikale wurden auch in den stoßinduzierten Fragmentierungsexperimenten der heteroleptischen Spezies $\mathrm{PhFeDec}_{3}{ }^{-}$und $\mathrm{MeFeDec}_{3}{ }^{-}$primär gebildet. Für die OrganoferratSerie Oct $_{4-n} \mathrm{FeDec}_{n}^{-}(n=1-3)$ wurden sowohl Decyl- als auch Octyl-Radikale unter Reduktion des Eisen-Kerns eliminiert. Die dppbz-haltigen Komplexe EtFe(dppbz)- und MeFe(dppbz) ${ }^{+}$ sowie die heterobimetallische Spezies $\mathrm{Me}_{8} \mathrm{LiFe}_{2}{ }^{-}$zeigten durch Abspaltung eines Methyl- bzw. Ethylradikals den analogen Fragmentierungskanal.

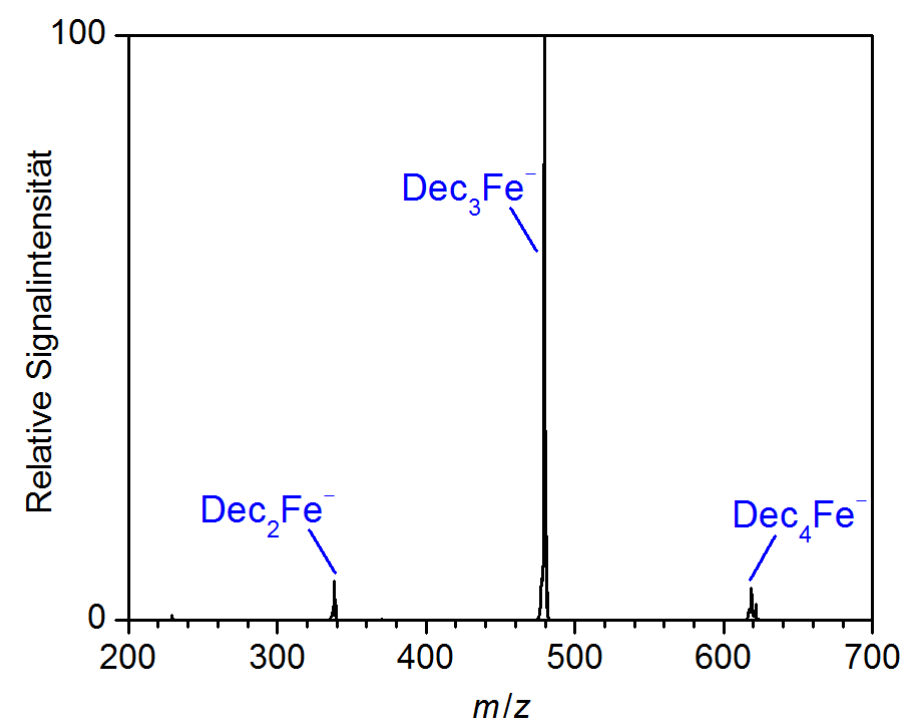

Abbildung 73. Massenspektrum von $\mathrm{Dec}_{4} \mathrm{Fe}^{-}(\mathrm{m} / z$ 621) nach Isolation und anschließender Fragmentierung über stoßinduzierte Dissoziation $\left(V_{\text {Anr }}=0.60 \mathrm{~V}\right)$. 


\subsection{3 $\beta$-H-Eliminierung}

Alkylferrate der allgemeinen Form $\mathrm{R}_{3} \mathrm{Fe}(\mathrm{II})^{-}(\mathrm{R}=\mathrm{Bu}$, Hex, Oct, Dec) bildeten in der Gasphase $\mathrm{R}_{2} \mathrm{Fe}(\mathrm{II}) \mathrm{H}^{-}$als Hauptprodukt der Fragmentierung. Diese Zerfallsreaktion entsprach höchstwahrscheinlich einer $\beta-\mathrm{H}$-Eliminierung unter Freisetzung des jeweiligen Alkens $(\rightarrow$ Schema 27).

$$
\mathrm{R}_{3} \mathrm{Fe}^{-} \longrightarrow \mathrm{R}_{2} \mathrm{FeH}^{-}+(\mathrm{R}-\mathrm{H})
$$

Schema 27. Unimolekulare Gasphasenreaktivität von $\mathrm{R}_{3} \mathrm{Fe}^{-}(\mathrm{R}=\mathrm{Bu}$, Hex, Oct, Dec) mit $\beta-\mathrm{H}$-Eliminierung und Bildung des entsprechenden Alkens.

Repräsentativ soll die unimolekulare Reaktivität von $\mathrm{Dec}_{3} \mathrm{Fe}(\mathrm{II})^{-}$gezeigt werden $\left(\rightarrow\right.$ Abbildung 74). Für diese war die Bildung der anionischen Spezies $\mathrm{Dec}_{2} \mathrm{Fe}(\mathrm{II}) \mathrm{H}^{-}$und $\operatorname{DecFe}(\mathrm{II}) \mathrm{H}_{2}{ }^{-}$über $\beta-\mathrm{H}$-Eliminierung charakteristisch. Hervorzuheben ist, dass im Gegensatz dazu die entsprechenden Eisen(III)-Spezies $\mathrm{R}_{4} \mathrm{Fe}^{-}(\mathrm{R}=\mathrm{Bu}$, Hex, Oct, Dec) alternative Fragmentierungskanäle aufwiesen (siehe Kapitel 4.3.1 und 4.3.2)

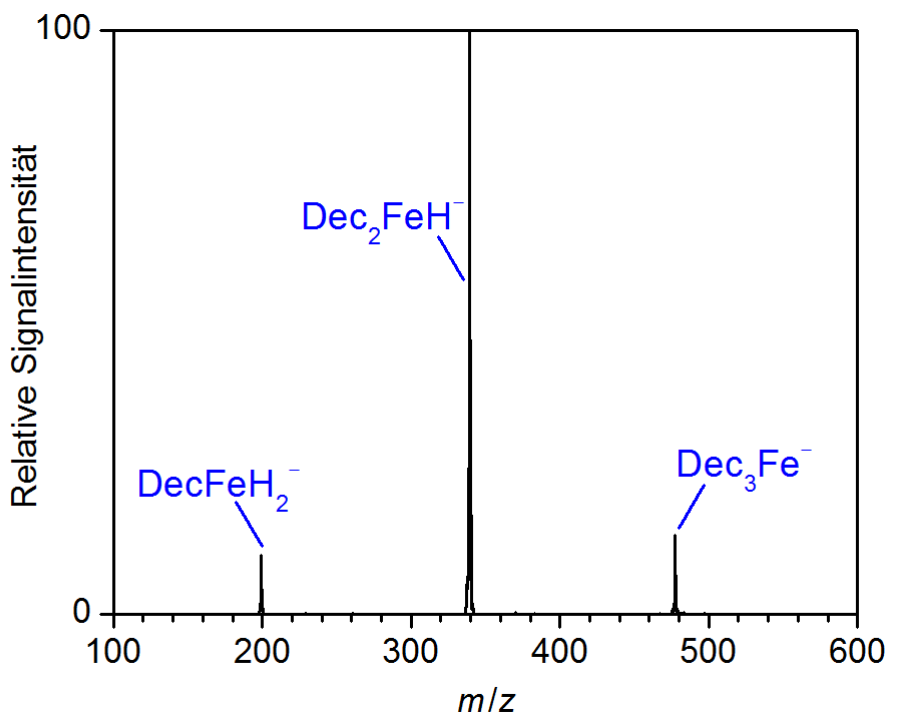

Abbildung 74. Massenspektrum von $\mathrm{Dec}_{3} \mathrm{Fe}^{-}(\mathrm{m} / \mathrm{z} 479)$ nach Isolation und anschließender Fragmentierung über stoßinduzierte Dissoziation $\left(V_{\text {Anr }}=0.70 \mathrm{~V}\right)$.

Eine analoges Verhalten zeigten die Komplexe $\mathrm{Bu}_{2} \mathrm{Fe}(\mathrm{II}) \mathrm{X}^{-}$und $\mathrm{BuFeX}_{2}^{-}(\mathrm{X}=\mathrm{Cl}, \mathrm{Br}$, I) sowie die heteroleptischen Organoferrate $\mathrm{Ph}_{2} \mathrm{FeDec}^{-}$und $\mathrm{PhFeDec}_{2}{ }^{-}$in der Gasphase, die allesamt über $\beta$-H-Eliminierung fragmentierten. 
Die $\beta$-H-Eliminierung als Fragmentierungsreaktion war nicht nur auf mononukleare Spezies beschränkt. So zeigte mit $\mathrm{Hex}_{5} \mathrm{Fe}_{2}^{-}$ein zweikerniges, homoleptisches Organoferrat den konsekutiven Zerfall über $\beta$-H-Eliminierung ( $\rightarrow$ Abbildung 75 und Schema 28). Auch der niedervalente Eisenkomplex $\mathrm{EtFe}_{2}(\mathrm{dppbz})^{-}$konnte durch stoßinduzierte Dissoziation mit Ethen das Produkt dieser Zerfallsreaktion freisetzen.

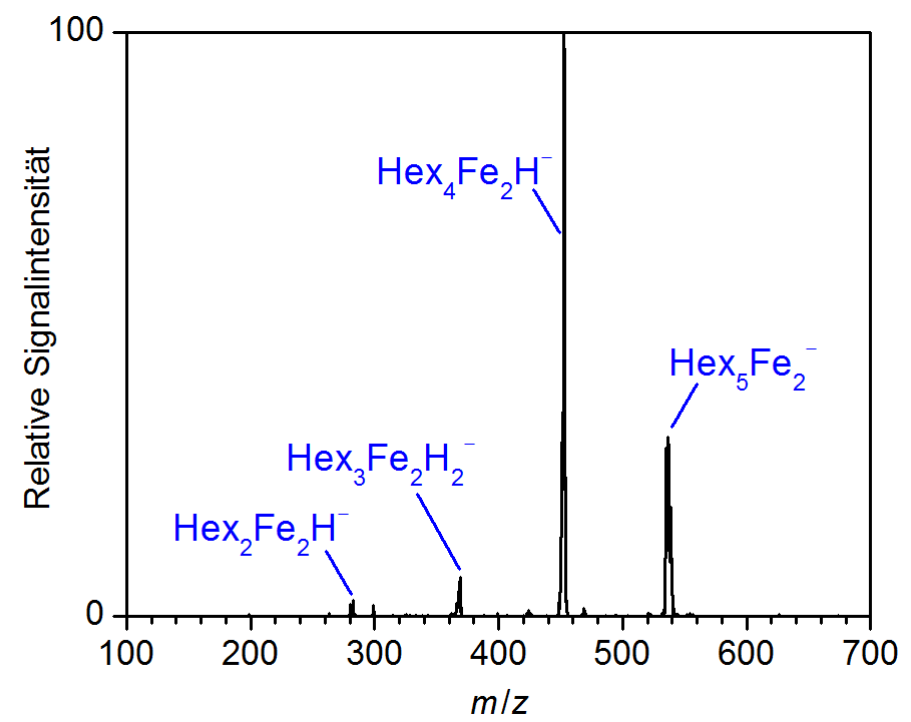

Abbildung 75. Massenspektrum von $\mathrm{Hex}_{5} \mathrm{Fe}_{2}{ }^{-}(\mathrm{m} / \mathrm{z} 537)$ nach Isolation und anschließender Fragmentierung über stoßinduzierte Dissoziation ( $\left.V_{\text {Anr }}=0.50 \mathrm{~V}\right)$.

$$
\begin{aligned}
& \mathrm{Hex}_{5} \mathrm{Fe}_{2}^{-} \longrightarrow \mathrm{Hex}_{4} \mathrm{Fe}_{2} \mathrm{H}^{-}+(\mathrm{R}-\mathrm{H}) \\
& \mathrm{Hex}_{4} \mathrm{Fe}_{2} \mathrm{H}^{-} \longrightarrow \mathrm{Hex}_{3} \mathrm{Fe}_{2} \mathrm{H}_{2}^{-}+(\mathrm{R}-\mathrm{H}) \\
& \mathrm{Hex}_{3} \mathrm{Fe}_{2} \mathrm{H}_{2}^{-} \longrightarrow \mathrm{Hex}_{2} \mathrm{Fe}_{2} \mathrm{H}_{3}^{-}+(\mathrm{R}-\mathrm{H})
\end{aligned}
$$

Schema 28. Unimolekulare Gasphasenreaktivität von $\mathrm{Hex}_{5} \mathrm{Fe}_{2}{ }^{-}$mit konsekutiven $\beta-\mathrm{H}-\mathrm{Eliminierungen} \mathrm{und}$ Bildungen der entsprechenden Alkene. Zu beachten ist, dass $\mathrm{Hex}_{2} \mathrm{Fe}_{2} \mathrm{H}_{3}{ }^{-}$nicht direkt beobachtet werden konnte. Ausgehend von $\mathrm{Hex}_{2} \mathrm{Fe}_{2} \mathrm{H}_{3}{ }^{-}$kann mit $\mathrm{Hex}_{2} \mathrm{Fe}_{2} \mathrm{H}^{-}$aber das Produkt einer $\mathrm{H}_{2}$-Eliminerung detektiert werden. 


\subsubsection{Freisetzung von $\mathrm{RH}$}

Einkernige, homoleptische Arylferrate $\mathrm{R}_{3} \mathrm{Fe}(\mathrm{II})^{-}(\mathrm{R}=\mathrm{Ph}$, Mes, o-Tol) zerfielen im stoßinduzierten Gasphasen-Fragmentierungsexperiment über Freisetzung von neutralen $\mathrm{RH}$ Molekülen ( $\rightarrow$ Schema 29 und Abbildung 76). So fragmentierte $\mathrm{Mes}_{3} \mathrm{Fe}(\mathrm{II})^{-}$über die Bildung von $\mathrm{C}_{9} \mathrm{H}_{12}(\mathrm{MesH})$. Offensichtlich war, dass diesem Zerfallskanal eine $\mathrm{C}-\mathrm{H}$-Aktvierung vorausgegangen sein musste.

$$
\mathrm{R}_{3} \mathrm{Fe}^{-} \longrightarrow\left[\mathrm{R}_{3} \mathrm{Fe}-\mathrm{RH}\right]^{-}+\mathrm{RH}
$$

Schema 29. Unimolekulare Gasphasenreaktivität von $\mathrm{R}_{3} \mathrm{Fe}^{-}(\mathrm{R}=\mathrm{Ph}$, Mes, o-Tol) mit Freisetzung eines neutralen RH-Moleküls.

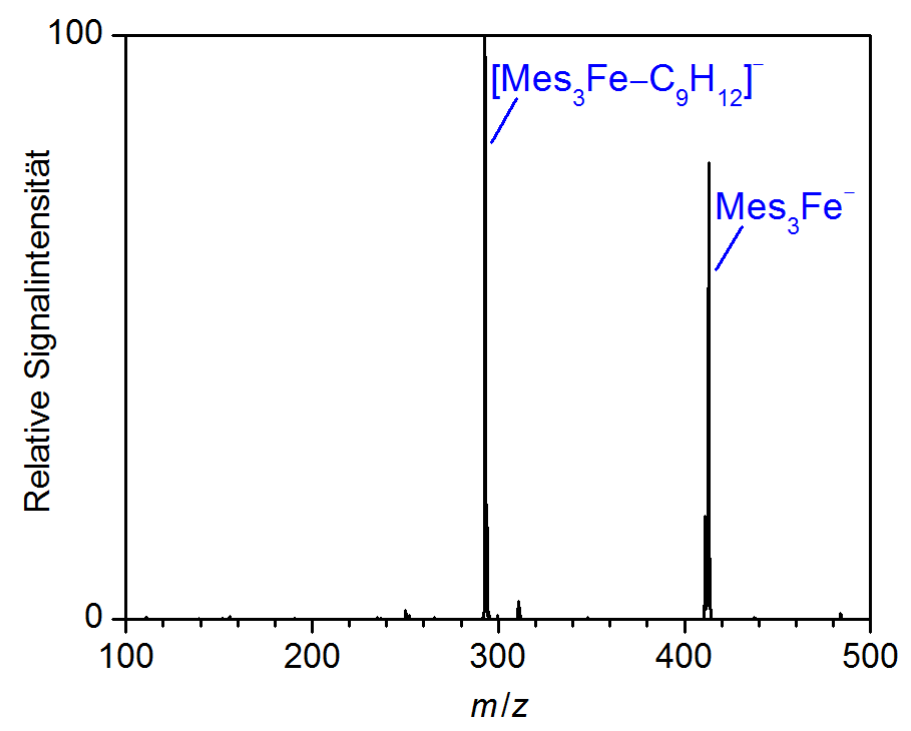

Abbildung 76. Massenspektrum von $\mathrm{Mes}_{3} \mathrm{Fe}^{-}(\mathrm{m} / \mathrm{z} 416)$ nach Isolation und anschließender Fragmentierung über stoßinduzierte Dissoziation $\left(V_{\text {Anr }}=0.80 \mathrm{~V}\right)$.

Analoge Zerfallskanäle waren für die Spezies $\mathrm{Ph}_{2} \mathrm{Fe}(0-\mathrm{Tol})^{-}, \mathrm{Ph}_{2} \mathrm{FeDec}^{-}, \mathrm{PhFeMes}_{2}{ }^{-}$sowie $\mathrm{Mes}_{2} \mathrm{FeBr}^{-} \mathrm{zu}$ beobachten. Auf eine ähnliche Weise fragmentierten die Eisen(IV)-Spezies $\mathrm{Ph}_{4} \mathrm{FeR}^{-}\left(\mathrm{R}^{\prime}=\mathrm{o}-\mathrm{Tol}, \quad 0-\mathrm{CF}_{3}-\mathrm{C}_{6} \mathrm{H}_{4}\right)$ in der Gasphase. So zeigten beide Spezies den konsekutiven Zerfall über Freisetzung zweier $\mathrm{C}_{6} \mathrm{H}_{6}$-Einheiten. Ausgehend von der anionischen Eisen(III)-Spezies $\left.\mathrm{Bu}_{4} \mathrm{Fe}_{2} \mathrm{H}_{2}\right|^{-}$entsprach auch der zweifache Verlust von Butan allgemein der Eliminierung von RH-Molekülen. Da die ursprüngliche Spezies bereits Hydrid-Substituenten aufwies, waren diese Reaktionskanäle aber als reduktive Eliminierungen zu verstehen. 


\subsubsection{Weitere Gasphasenreaktionen}

Neben den bereits erwähnten Gasphasenreaktivitäten traten für die Organoferrate weitere Fragmentierungskanäle auf. Für nahezu alle dppbz- und $\mathrm{PPh}_{3}$-haltigen Eisen-Spezies war, unabhängig vom Aggregationsgrad und von der Oxidationsstufe des Eisen-Zentrums, die Zersetzung des Liganden in der Gasphase zu beobachten. So konnten Eisen-Komplexe wie $\mathrm{Ph}_{3} \mathrm{Fe}_{2} \mathrm{~L}^{-}\left(\mathrm{L}=\mathrm{dppbz}, \mathrm{PPh}_{3}\right)$ bei einer Stoßamplitude von $V_{\mathrm{Anr}}=0.7 \mathrm{~V}$ über Freisetzung von $\mathrm{Ph}_{2}$ fragmentieren ( $\rightarrow$ Abbildung 77 und Schema 30). Dass dieser Reaktionskanal zweifach auftrat, das ursprüngliche Anion aber nur über drei Phenyl-Substituenten verfügte, deutete auf eine Dekomposition des Liganden hin. Auch konnte der Ligand zusätzlich über eine Benzolabspaltung fragmentieren. Im Gegensatz dazu dissoziierte $\left(\mathrm{Ar}_{\mathrm{F}}\right)_{3} \mathrm{Fe}(\mathrm{dppbz})^{-}$ $\left(\mathrm{Ar}_{\mathrm{F}}=3,5-\left(\mathrm{CF}_{3}\right)_{2}-\mathrm{C}_{6} \mathrm{H}_{3}\right)$ bei einer Anregungsspannung $V_{\mathrm{Anr}}$ von nur $0.25 \mathrm{~V}$ den intakten Liganden in der Gasphase ( $\rightarrow$ Abbildung 78 und Schema 31).

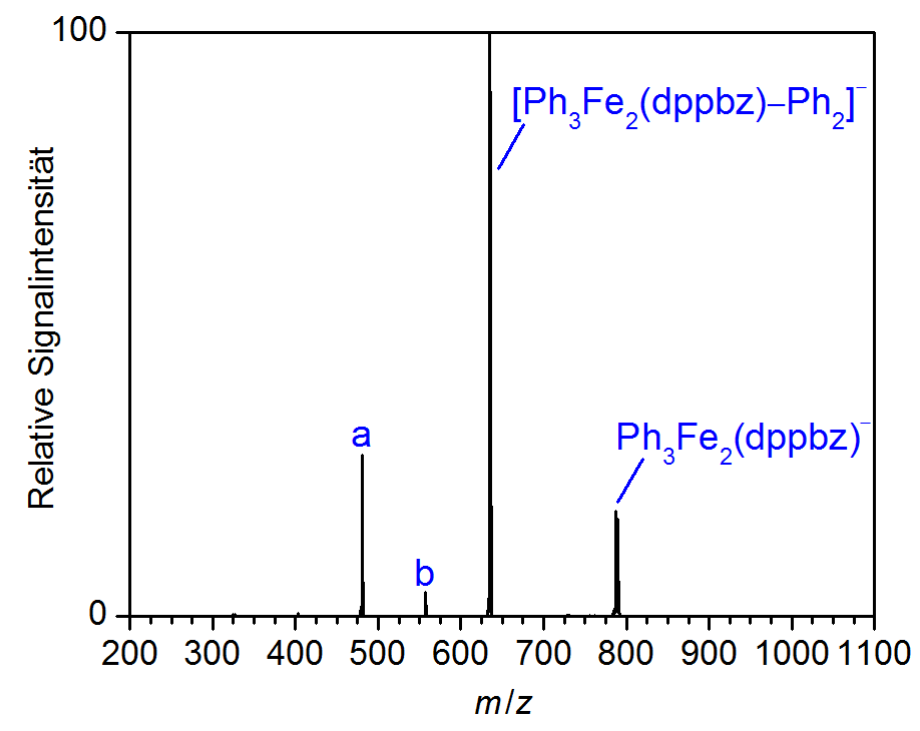

Abbildung 77. Massenspektrum von $\mathrm{Ph}_{3} \mathrm{Fe}_{2}(\mathrm{dppbz})^{-}(\mathrm{m} / \mathrm{z}$ 789) nach Isolation und anschließender Fragmentierung über stoßinduzierte Dissoziation $\left(V_{A n r}=0.70 \mathrm{~V}\right) ; \mathrm{a}=\left[\mathrm{Ph}_{3} \mathrm{Fe}_{2}(\mathrm{dppbz})-2 \mathrm{Ph}_{2}\right]^{-}, \mathrm{b}=\left[\mathrm{Ph}_{3} \mathrm{Fe}_{2}(\mathrm{dppbz})-\mathrm{C}_{18} \mathrm{H}_{16}\right]^{-}$.

$$
\begin{aligned}
& \mathrm{Ph}_{3} \mathrm{Fe}_{2} \mathrm{~L}^{-} \longrightarrow\left[\mathrm{Ph}_{3} \mathrm{Fe}_{2} \mathrm{~L}-\mathrm{Ph}_{2}\right]^{-}+\mathrm{Ph}_{2} \\
& \mathrm{Ph}_{3} \mathrm{Fe}_{2} \mathrm{~L}^{-} \longrightarrow\left[\mathrm{Ph}_{3} \mathrm{Fe}_{2} \mathrm{~L}-\mathrm{C}_{18} \mathrm{H}_{16}\right]^{-}+\mathrm{Ph}_{2}+\mathrm{C}_{6} \mathrm{H}_{6} \\
& \mathrm{Ph}_{3} \mathrm{Fe}_{2} \mathrm{~L}^{-} \longrightarrow\left[\mathrm{Ph}_{3} \mathrm{Fe}_{2} \mathrm{~L}-2 \mathrm{Ph}_{2}\right]^{-}+2 \mathrm{Ph}_{2}
\end{aligned}
$$

Schema 30. Unimolekulare Gasphasenreaktivität von $\mathrm{Ph}_{3} \mathrm{Fe}_{2} \mathrm{~L}^{-}\left(\mathrm{L}=\mathrm{dppbz}, \mathrm{PPh}_{3}\right)$ mit reduktiver Eliminierung von $\mathrm{Ph}_{2}$ und Abspaltung von $\mathrm{C}_{6} \mathrm{H}_{6}$. Die Abspaltungsprodukte deuteten auf eine Zersetzung des Liganden $\mathrm{L}$ hin. 


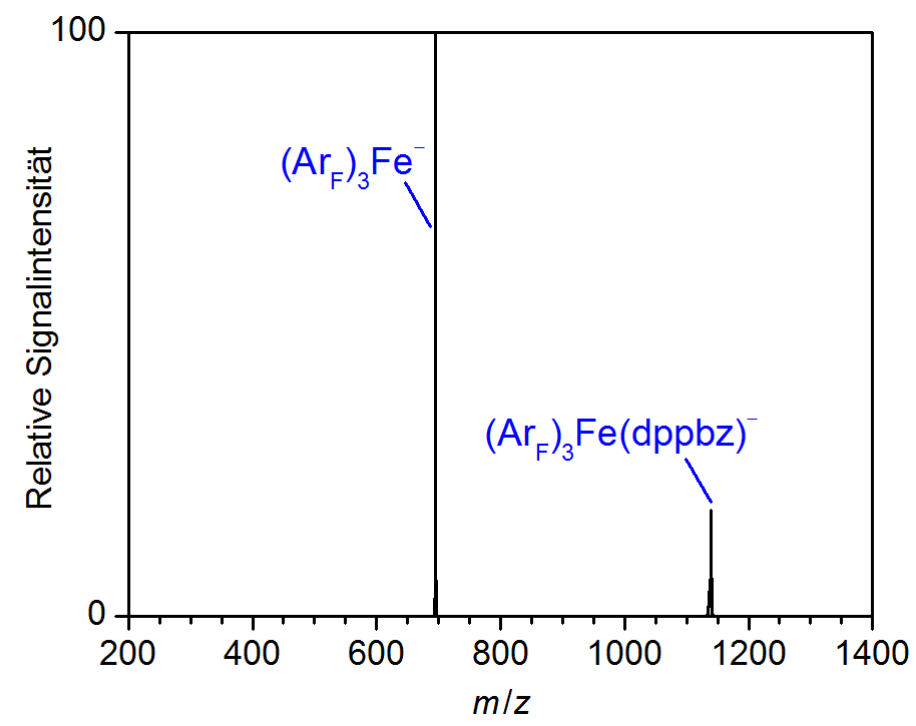

Abbildung 78. Massenspektrum von $\left(\mathrm{Ar}_{\mathrm{F}}\right)_{3} \mathrm{Fe}(\mathrm{dppbz})^{-}(\mathrm{m} / \mathrm{z} 1141)$ nach Isolation und anschließender Fragmentierung über stoßinduzierte Dissoziation ( $\left.V_{\text {Anr }}=0.25 \mathrm{~V}\right)$.

$$
\left(\mathrm{Ar}_{\mathrm{F}}\right)_{3} \mathrm{Fe}(\mathrm{dppbz})^{-} \longrightarrow\left(\mathrm{Ar}_{\mathrm{F}}\right)_{3} \mathrm{Fe}^{-}+\mathrm{dppbz}
$$

Schema 31. Unimolekulare Gasphasenreaktivität von $\left(\mathrm{Ar}_{\mathrm{F}}\right)_{3} \mathrm{Fe}(\mathrm{dppbz})^{-}$mit Dissoziation des intakten Liganden dppbz.

Neben den bereits erwähnten radikalischen Prozessen waren für $\mathrm{Me}_{12} \mathrm{Fe}_{8}{ }^{-}$und artverwandte Cluster-Anionen vielfältige Fragmentierungspfade von Bedeutung. Viele der Reaktionskanäle und Fragmentionen waren sehr komplex und ließen sich erst nach Vergleich mit der Gasphasenreaktivität von $\left(\mathrm{CD}_{3}\right)_{12} \mathrm{Fe}_{8}{ }^{-}$entschlüsseln ( $\rightarrow$ Abbildung 79 ). So konnten Spezies der allgemeinen Form $\left[\mathrm{Fe}_{8}, \mathrm{C}_{9}, \mathrm{D}_{24}\right]^{-},\left[\mathrm{Fe}_{8}, \mathrm{C}_{10}, \mathrm{D}_{27}\right]^{-}$und $\left[\mathrm{Fe}_{8}, \mathrm{C}_{11} \mathrm{D}_{30}\right]^{-}$eindeutig als Fragmente von $\left(\mathrm{CD}_{3}\right)_{12} \mathrm{Fe}_{8}^{-} /\left[\mathrm{Fe}_{8}, \mathrm{C}_{12}, \mathrm{D}_{36}\right]^{-}$identifiziert werden. Markant war zudem die Spezies $\left(\mathrm{CD}_{3}\right)_{12} \mathrm{Fe}_{7}{ }^{-}$, dessen Bildung auf eine Freisetzung von $\mathrm{Fe}\left(\mathrm{CD}_{3}\right)_{2}$ hindeutete. Fragmentierung über Abspaltung neutraler Produkte der allgemeinen Form FeR $\mathrm{F}_{2}$ waren für Organoferrate nicht ungewöhnlich ( $\rightarrow$ Schema 32). So zeigten auch die Spezies $\mathrm{R}_{5} \mathrm{Fe}_{2}^{-}\left(\mathrm{R}=\mathrm{Bn}, \mathrm{Me}_{3} \mathrm{SiCH}_{2}\right)$ und die hochaggregierte Spezies $\mathrm{Me}_{18} \mathrm{Fe}_{12}{ }^{-}$diesen charakteristischen Zerfallskanal $(\rightarrow$ Abbildung 80). 


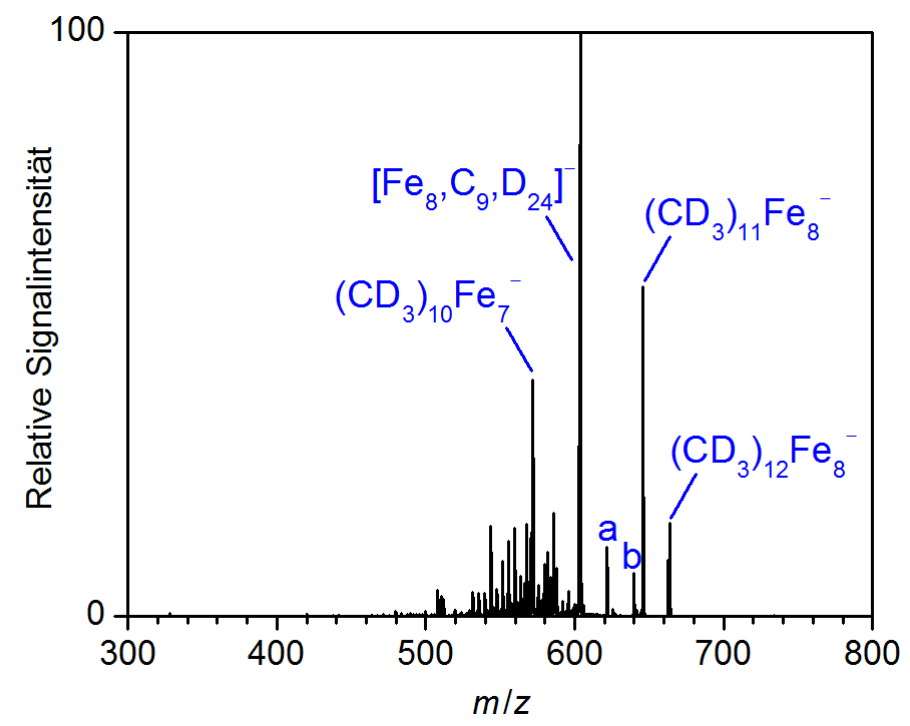

Abbildung 79. Massenspektrum von $\left(\mathrm{CD}_{3}\right)_{12} \mathrm{Fe}_{8}{ }^{-}(\mathrm{m} / \mathrm{z} 664)$ nach Isolation und anschließender Fragmentierung über stoßinduzierte Dissoziation $\left(V_{\text {Anr }}=0.70 \mathrm{~V}\right), a=\left[\mathrm{Fe}_{8}, \mathrm{C}_{10}, \mathrm{D}_{27}\right]^{-}, \mathrm{b}=\left[\mathrm{Fe} \mathrm{C}_{8}, \mathrm{C}_{11}, \mathrm{D}_{30}\right]^{-}$.

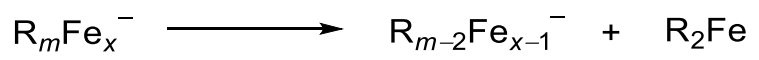

Schema 32. Allgemeines Reaktionsschema für die unimolekulare Gasphasenreaktivität von $\mathrm{R}_{m} \mathrm{Fe}_{x}{ }^{-}$mit Freisetzung von $\mathrm{R}_{2} \mathrm{Fe}$.

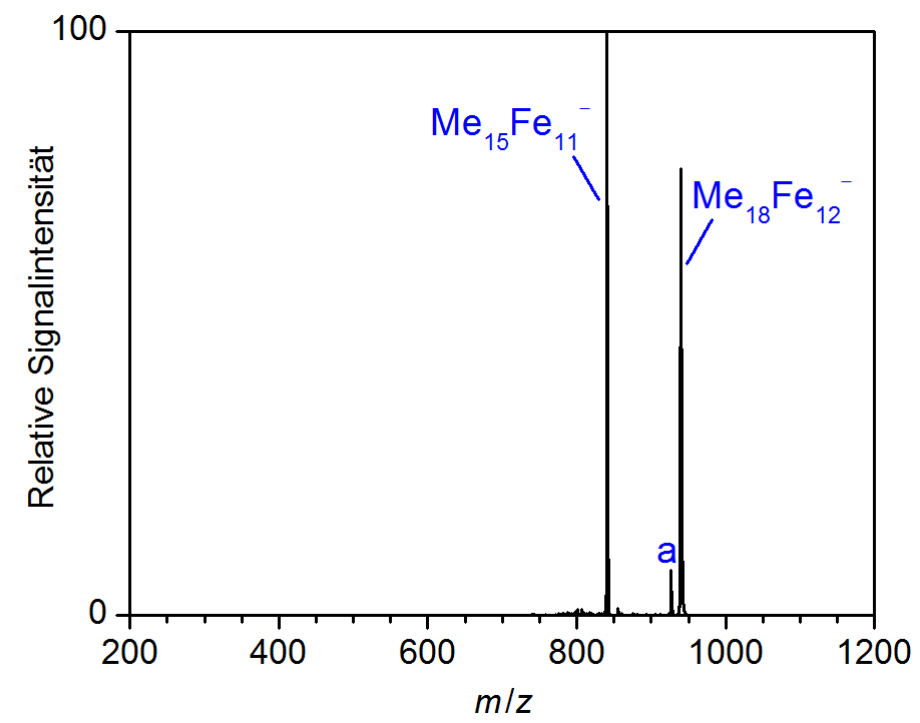

Abbildung 80. Massenspektrum von $\mathrm{Me}_{18} \mathrm{Fe}_{12}{ }^{-}(\mathrm{m} / \mathrm{z}$ 942) nach Isolation und anschließender Fragmentierung über stoßinduzierte Dissoziation $\left(V_{\text {Anr }}=0.70 \mathrm{~V}\right), a=\mathrm{Me}_{17} \mathrm{Fe}_{12}{ }^{-}$. 


\subsubsection{Unimolekulare Gasphasenreaktivität der Organoferrate im Vergleich}

Experimente auf der Basis von stoßinduzierten Dissoziationen massenselektierter EisenSpezies in der Gasphase demonstrierten, dass sowohl Ein- als auch Zwei-ElektronenProzesse in der Organoeisen-Chemie von Bedeutung sind. Für die Eisen(III)-Spezies $\mathrm{R}_{4} \mathrm{Fe}^{-}$ $(\mathrm{R}=\mathrm{Alkyl})$ waren vor allem radikalische Zerfallsprozesse sehr prominent. Hingegen war für die Arylferrate $\mathrm{R}_{4} \mathrm{Fe}(\mathrm{III})^{-}$ausschließlich die reduktive Eliminierung als Zwei-ElektronenReaktion in der Gasphase zu beobachten, was die vergleichsweise geringe Stabilität der Arylradikale widerspiegelt. Für die Serie heteroleptischer Tetraarylferrate $\mathrm{R}_{3} \mathrm{Fe}(\mathrm{III}) \mathrm{R}^{\prime-}$ wurden elektronische Einflüsse auf die reduktive Eliminierung in der Gasphase im Detail untersucht. So zeigte sich, dass die Anwesenheit elektronenreicher Substituenten R' zu einer Dominanz des Kreuzkupplungskanales führte, wohingegen elektronenarme Systeme primär über Freisetzung des Homokupplungsproduktes fragmentierten. Während der reduktiven Eliminierung wird das Eisen(III)-Zentrum auf die elektronenreichere Oxidationsstufe +I reduziert. Zieht man zusätzlich den anionischen Charakter der Organoferrate in Betracht, so scheint der Aufbau zusätzlicher Elektronendichte am Eisenzentrum energetisch ungünstig zu sein. Delokalisierung der resultierenden Elektronendichte durch elektronenziehende Substituenten hilft, die elektronenreichen Eisen-Zentren zu stabilisieren. Somit bleiben generell elektronenärmere Arylreste während der reduktiven Eliminierung an das EisenZentrum gebunden, während die elektronenreicheren Systeme bevorzugt als Kupplungsprodukt freigesetzt werden. Der resultierende Hammett-Plot zeigt eine durchweg negative Steigung, was auf einen ähnlichen, zugrunde liegenden Reaktionsmechanismus für die Freisetzung der Kupplungsprodukte hindeutet. Nichtsdestotrotz belegt die steiler werdende, negative Steigung für elektronenziehende Substituenten, dass eine Änderung im Reaktionsmechanismus erfolgt. Eine mögliche Erklärung ergibt sich durch Berücksichtigung der Tatsache, dass die reduktive Eliminierung an sich ein Reaktionsprozess ist, der in zwei Schritten erfolgt ( $\rightarrow$ Schema 33). Im ersten Reaktionsschritt wird das Produkt der reduktiven Eliminierung am Eisen-Zentrum vorgebildet. Abschließend dissoziiert der Komplex und setzt das Kupplungsprodukt $R_{2}$ frei. Eine Veränderung der elektronischen Eigenschaften am Eisenkomplex führt dazu, dass die beiden relevanten Energiebarrieren relativ zueinander verschoben werden. Wahrscheinlich ist, dass elektronenziehende Substituenten das resultierende Intermediat $\mathrm{PhFeR}^{\prime}\left(\mathrm{Ph}_{2}\right)^{-}$stabilisieren, die abschließende Dissoziation erschweren und damit diesen Reaktionsschritt als geschwindigkeitsbestimmend für die gesamte reduktive Eliminierung festsetzen. So spiegelt die steiler werdende Steigung des Hammett-Plots die Empfindlichkeit des Dissoziationsschrittes gegenüber elektronischen Effekten wider. 


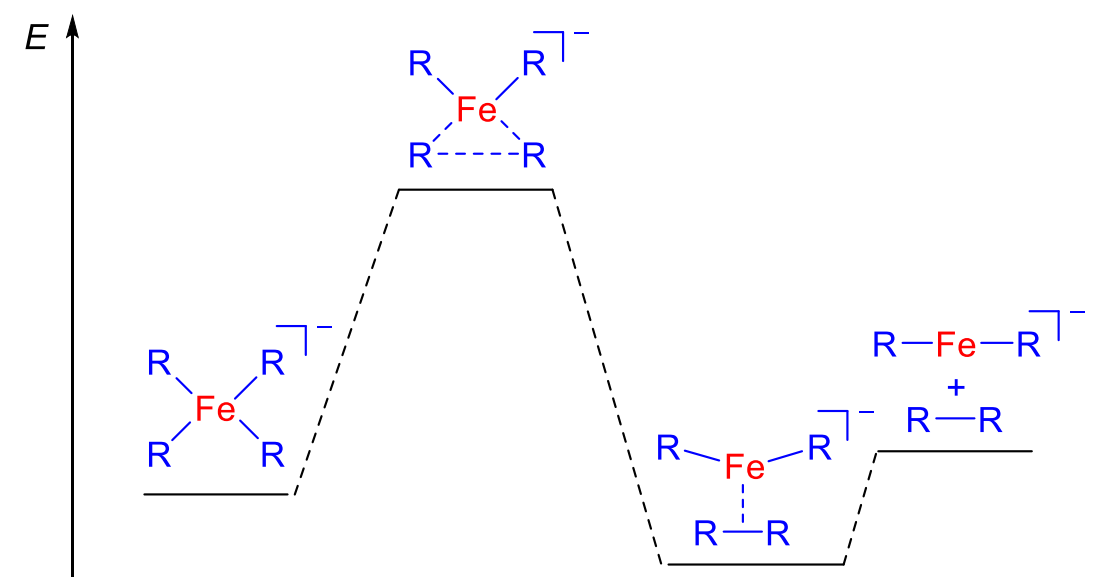

Schema 33. Schematisches Energieprofil des Ablaufs der reduktiven Eliminierung von massenselektierten Tetraarylferraten.

Die elektronischen Effekte, die sich auf das Fragmentierungsverhalten der Tetraarylferrate auswirken, spiegeln sich auch in der Gasphasenreaktivität heteroleptischer Spezies des Typs $\mathrm{Ph}_{3} \mathrm{FeR}^{\prime-} \quad\left(\mathrm{R}^{\prime}=\mathrm{Alkyl}\right)$ wider. So verbleiben die stärker elektronenziehenden PhenylSubstituenten bevorzugt am Eisen-Zentrum, wohingegen die vergleichsweise weniger elektronenziehenden Alkyl-Reste durch reduktive Eliminierung des Kreuzkupplungsproduktes den Eisen-Komplex verlassen. Ein ähnliches Gasphasen-Verhalten konnte in der Vergangenheit bereits für heteroleptische Alkylcuprate mit Kupfer(III)-Zentren beobachtet werden. ${ }^{[64,96]}$ Auch die Tatsache, dass $\mathrm{Ph}_{3} \mathrm{FeBn}^{-}$in der Gasphase mit $\mathrm{Ph}_{2}$ primär das Homokupplungsprodukt eliminiert, befindet sich mit diesem Erklärungsansatz in Übereinstimmung. Aus den entsprechenden Gasphasen-Aciditäten $\left(\Delta H_{\text {Acid }}(\mathrm{BnH})=1592 \mathrm{~kJ} \mathrm{~mol}^{-1}\right.$, $\left.\Delta H_{\text {Acid }}(\mathrm{PhH})=1669 \mathrm{~kJ} \mathrm{~mol}^{-1}\right)^{[97]}$ geht hervor, dass Benzyl-Substituenten besser in der Lage sind, zusätzliche Elektronendichte zu stabilisieren als Ph-Reste.iii

Theoretische Rechnungen bezogen auf neutrale Komplexe des Typs $L_{2} M R_{2}\left(L=P_{3} ; M=N i\right.$, $\mathrm{Pd}, \mathrm{Pt} ; \mathrm{R}=\mathrm{H}, \mathrm{Me}$ ) deuten auf vergleichsweise geringe Aktivierungsenergien für die reduktive Eliminierung elektronenreicher Substituenten $R$ hin. ${ }^{[98]}$ Nach Aussage von Hartwig und Mitarbeiter spiegelt dies die stärkere Bindung elektronenziehender Substituenten an das Metallzentrum wider, wodurch der ionische Charakter der M-R-Bindung erhöht ist. ${ }^{[99]}$ Weitere Arbeiten zeigten, dass in C-C- und C-N-Kupplungsreaktionen mit Palladium- und PlatinKomplexen die Elektronendichte am Metallzentrum nicht entscheidend dafür ist, welches

\footnotetext{
iii Überraschend war, dass $\mathrm{Ph}_{2} \mathrm{FeBn}_{2}{ }^{-}$im Gasphasen-Fragmentierungsexperiment reduktiv $\mathrm{Bn}_{2}$ eliminierte. Hierfür konnte abschließend keine Erklärung gefunden werden.
} 
Produkt reduktiv eliminiert wird. ${ }^{[99,100]}$ Vielmehr scheinen die unterschiedlichen elektronischen Eigenschaften der Substituenten eine entscheidende Rolle zu spielen. Im Vergleich mit den heteroleptischen Organoferraten $\mathrm{Ph}_{3} \mathrm{FeR}^{-}$ist deren anionischer Charakter von hoher Bedeutung. So sollte in diesem Fall der elektronenziehende Effekt eines Substituenten einen viel größeren Einfluss auf die reduktive Eliminierung haben, als für die neutralen Palladiumund Platin-Komplexe zu beobachten war.

Neben den elektronischen Einflüssen wurden die sterischen Effekte auf die reduktive Eliminierung der heteroleptischen Tetraarylferrate $\mathrm{Ph}_{3} \mathrm{FeR}^{-}$in der Gasphase untersucht. So verblieb der sterisch anspruchsvollere Substituent im Fragmentierungsexperiment primär am Eisen-Zentrum. Infolgedessen war die Abspaltung des Homokupplungsproduktes $\mathrm{Ph}_{2}$ in diesen Fällen stets der Hauptzerfallsweg. In der für die reduktive Eliminierung relevanten Übergangsstruktur kann im Wesentlichen zwischen zwei repulsiven sterischen Wechselwirkungen unterschieden werden:

a) Die vier aromatischen Substituenten am Eisenzentrum stoßen sich voneinander ab. Diese Wechselwirkung sollte für frühe Übergangsstrukturen besonders ausgeprägt sein, in denen die beiden zu eliminierenden Aryl-Reste nahe am Eisen-Zentrum vorliegen.

b) Die beiden Aryl-Gruppen, die die neue C-C-Bindung ausbilden, beeinflussen sich gegenseitig sterisch. So kommen sich die beiden Aryl-Substituenten vor allem in einer späten Übergangsstruktur räumlich sehr nahe und stoßen sich voneinander ab.

Wenn die Wechselwirkung zwischen den vier Aryl-Substituenten dominiert, ist zu erwarten, dass der sterisch anspruchsvollste Substituent primär eliminiert wird. Im Gegensatz dazu sollte dieser am Eisen-Zentrum verbleiben, wenn die sterische Wechselwirkung zwischen den zu dissoziierenden Aryl-Gruppen entscheidend ist. Die experimentellen Daten stimmen mit dem zweiten Szenario überein, sodass im Fall der reduktiven Eliminierung der heteroleptischen Organoferrate $\mathrm{Ph}_{3} \mathrm{FeR}^{-}$ein später Übergangszustand anzunehmen ist.

Arylferrate mit Eisen in der Oxidationsstufe +ll tendierten weniger dazu, über reduktive Eliminierung zu dissoziieren. Dies ist nicht überraschend, da die reduktive Eliminierung von Kupplungsprodukten bei elektronenreicheren Oxidationsstufen von Übergangsmetallen generell erschwert ist. ${ }^{[101]}$ Mit der Abspaltung von Arenen $\mathrm{R}-\mathrm{H}$ manifestierte sich für die Arylferrate $\mathrm{R}_{3} \mathrm{Fe}(\mathrm{II})^{-}$in der Gasphase ein Fragmentierungsprozess, der in Lösung bisher noch unbekannt ist.

Alkylferrate des Typs $\mathrm{R}_{4} \mathrm{Fe}^{-}(\mathrm{R}=\mathrm{Bu}$, Hex, Oct, Dec) zeigten einen prägnanten Zerfall durch Freisetzung von Alkylradikalen. Zusätzlich war in Spuren die Bildung der Spezies $\mathrm{R}_{2} \mathrm{Fe}(\mathrm{I})^{-} \mathrm{zu}$ 
beobachten, die aber nicht zwangsläufig aus einer reduktiven Eliminierung von $\mathrm{R}_{2}$ entstanden sein muss. Auch eine konsekutive Abspaltung aliphatischer Radikale kann deren Entstehung erklären. Da analoge Spezies des Typs $\mathrm{R}_{3} \mathrm{Fe}(\mathrm{II})^{-}$ausschließlich über $\beta-\mathrm{H}$-Eliminierung fragmentierten, kann letztgenannte Erklärung jedoch ausgeschlossen werden. Dass die $\beta$-H-Eliminierung grundsätzlich nicht für aliphatische Organoferrate $\mathrm{R}_{4} \mathrm{Fe}^{-}$mit Eisen(III)Zentrum auftrat, lässt sich auf deren vergleichsweise höhere Koordinationszahl zurückführen. So scheint im Falle dieser Spezies das Besetzen einer weiteren Koordinationsstelle mit der zu eliminierenden Alken-Spezies ungünstiger zu sein als für die analogen Eisen(II)-Komplexe.

$\mathrm{Me}_{12} \mathrm{Fe}_{8}{ }^{-}$und andere artverwandte Cluster-Anionen zeigten vielfältige, sehr komplexe Fragmentierungsprozesse. Die Bildung der Fragmente des achtkernigen Komplexes kann auf verschiedene Weise interpretiert werden ( $\rightarrow$ Schema 34). So ist neben der Eliminierung von intakten Methyl-Radikalen die Abspaltung von molekularem Wasserstoff und/oder Methan als sehr wahrscheinlich anzunehmen. Die dabei entstehenden Fragmente des allgemeinen Typs $\mathrm{MexFe}_{\mathrm{y}}\left(\mathrm{CH}_{2}\right)^{-}$können als Carben-Spezies verstanden werden. So haben in der Vergangenheit Armentrout und Mitarbeiter erfolgreich nachgewiesen, dass Fe+-Cluster das Potential haben, mit Methan zu reagieren und dabei Carben-Spezies auszubilden. ${ }^{102]}$

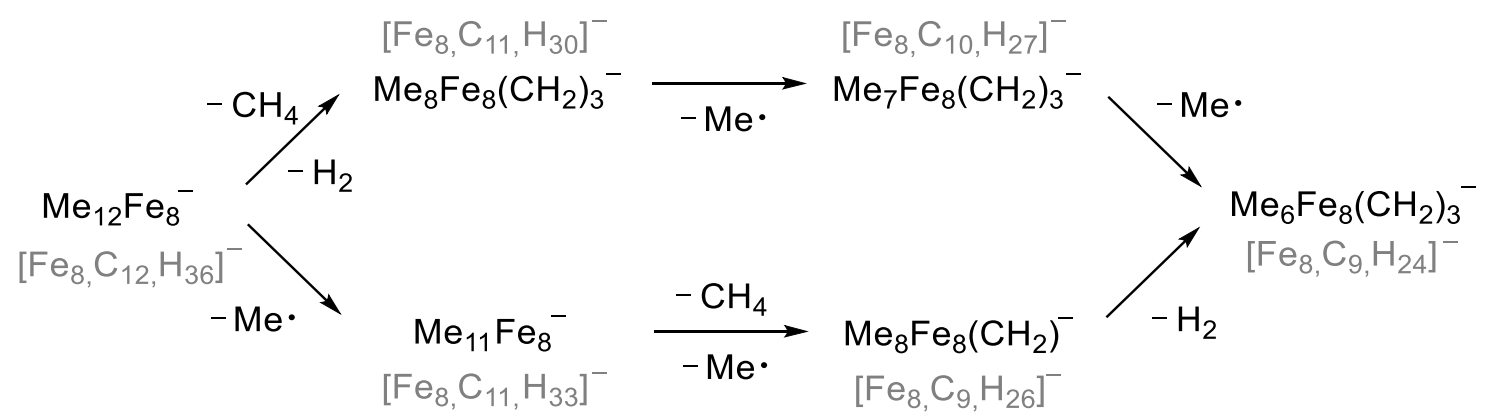

Schema 34. Mögliche Reaktionspfade, die die Bildung der beobachteten Fragment-Ionen aus dem stoßinduzierten Gasphasen-Fragmentierungsexperiment von $\mathrm{Me}_{12} \mathrm{Fe}_{8}^{-}$erklären können. 


\subsection{Katalysezyklus}

Zum jetzigen Standpunkt können die Ergebnisse aus dieser Arbeit genutzt werden, um einen alternativen Katalysezyklus für die ligandfreie Eisen-katalysierte Kreuzkupplung von GrignardReagenzien RMgX mit organischen Substraten R'X aufzustellen. So ist es sogar möglich, einen Katalysezyklus zu entwickeln, der vollständig auf Organoferraten als Reaktionsintermediaten beruht. Röntgen-Kristallstrukturanalysen aus den letzten fünf Jahren deuteten vermehrt auf eine mögliche Beteiligung dieser anionischen Eisen-Komplexe in den Reaktionsabläufen hin. ${ }^{[35 b, 38,39]}$ Diese Spezies waren bisher aber nur oberflächlich als mögliche Reaktionsintermediate untersucht und diskutiert worden. So konnte im Rahmen dieser Arbeit erstmals detailliert gezeigt werden, welch wichtige Rolle die Organoferrate im zugrunde liegenden Katalysezyklus spielen.

Die Eisen-katalysierte Kreuzkupplung von $\mathrm{PhMgX}$ mit aliphatischen oder aromatischen Substraten wurde im Verlauf dieser Arbeit am detailliertesten untersucht und soll in Form eines Katalysezyklus als Grundlage für andere Systeme genutzt werden, um mechanistische Gemeinsamkeiten und Unterschiede aufzudecken und zu verdeutlichen. Über Transmetallierung und Reduktion entstanden, unabhängig von der Natur des EisenPräkatalysators, Phenylferrate. Vor dem Hintergrund aller untersuchten Transmetallierungssysteme ist es mehr als wahrscheinlich, dass primär $\mathrm{Ph}_{3} \mathrm{Fe}(\mathrm{II})^{-}$über diesen Reaktionsprozess gebildet wird. Abhängig vom sterischen Anspruch der organischen Reste neigen die Eisen(II)At-Komplexe zur Disproportionierung. Aufgrund der sterisch vergleichsweise wenig anspruchsvollen Phenyl-Substituenten ist für $\mathrm{Ph}_{3} \mathrm{Fe}(\mathrm{II})^{-}$eine schnelle Disproportionierung zu beobachten, in deren Verlauf $\mathrm{Ph}_{4} \mathrm{Fe}(\mathrm{III})^{-}$gebildet wird. Gleichzeitig muss in diesem Reaktionsschritt mit $\mathrm{Ph}_{2} \mathrm{Fe}(\mathrm{I})^{-}$eine weitere Eisen-Spezies entstehen. Dass diese im Falle der Phenylferrate nicht beobachtet werden konnte, spiegelt die hohe Empfindlichkeit dieser elektronenreichen Spezies gegenüber ungewollten Nebenreaktionen wider. Insbesondere scheint $\mathrm{Ph}_{2} \mathrm{Fe}(\mathrm{I})^{-}$zur Überreduktion zu neigen, welche sich durch die Bildung eines niedervalenten, eisenhaltigen Feststoffes ausdrückt. Mono- und bidentate Phosphanliganden können entsprechende Eisen(I)-Spezies und andere niedervalente Eisen-Komplexe soweit stabilisieren, dass diese mittels ESI-Massenspektrometrie erfolgreich nachgewiesen werden konnten. Da solche Liganden in Eisen-katalysierten Kreuzkupplungsreaktionen regelmäßig Anwendung finden (siehe Kapitel 1.2), ist eine katalytische Aktivität von Spezies mit Eisenzentren in der Oxidationsstufe $\leq 1 \mathrm{sehr}$ wahrscheinlich. Mit TMEDA wurde zudem ein Additiv untersucht, dessen Präsenz dafür bekannt ist, die Ausbeute an Kreuzkupplungsprodukten in den entsprechenden Eisen-katalysierten Reaktionen zu erhöhen (siehe Kapitel 1.2). Alle Hinweise aus der Literatur und aus dieser Arbeit deuten darauf hin, dass TMEDA 
eine sekundäre Rolle im zugrunde liegenden Katalysezyklus spielt. ${ }^{[82]}$ So bindet TMEDA nicht an Eisen-Spezies, sondern an Magnesium-Kationen und beeinflusst durch andersartige Anionen-Kationen-Wechselwirkungen die Stabilität von $\mathrm{Ph}_{3} \mathrm{Fe}(\mathrm{II})^{-}$auf eine indirekte Art und Weise. Als Folge der Stabilitätserhöhung laufen die Disproportionierung und damit die Bildung von $\mathrm{Ph}_{2} \mathrm{Fe}(\mathrm{I})^{-}$deutlich langsamer ab. Auch ist denkbar, dass durch TMEDA indirekt ungewollte Überreduktionsprozesse eingeschränkt werden und mehr an katalytisch wirksamen EisenKomplexen für den nachfolgenden Kreuzkupplungsprozess zur Verfügung stehen. Als zusätzlichen Effekt bewirkt TMEDA eine Reduzierung des Aggregationsgrades von Organoferraten, sodass eine katalytische Aktivität mehrkerniger Aggregate ausgeschlossen werden kann.

Die hohe Reaktivität niedervalenter Phenylferrate gegenüber Organylhalogeniden konnte in bimolekularen Gasphasenexperimenten verdeutlicht werden. So zeigte speziell $\mathrm{Ph}_{2} \mathrm{Fe}(\mathrm{I})^{-}$eine ausgeprägte Reaktivität gegenüber Organyliodiden R'l, was die Vermutung nahelegt, dass $\mathrm{Ph}_{2} \mathrm{Fe}(\mathrm{I})^{-}$die höchste katalytische Bedeutung aller Phenylferrate hat. In der Gasphasenreaktion von $\mathrm{Ph}_{2} \mathrm{Fe}(\mathrm{I})^{-}$mit $\mathrm{R}^{\prime}$ l bildete sich mit $\mathrm{Ph}_{2} \mathrm{Fe}(\mathrm{II}) \mathrm{I}^{-}$ein Eisen-At-Komplex, der als das Zwischenprodukt einer schrittweise ablaufenden oxidativen Addition angesehen werden kann. ${ }^{[38]}$ Eine Detektion von $\mathrm{Ph}_{2} \mathrm{FeR}^{\prime}{ }^{-}$als Produkt des zweiten Schrittes der oxidativen Addition ist in der Gasphase nicht möglich. Die radikalische Natur des Additionsprozesses belegt zudem, wieso speziell Aryl-Aryl-Kreuzkupplungsreaktionen Eisen-katalysiert nur schwer zugänglich sind und nur geringe Ausbeuten an Kreuzkupplungsprodukt mit Hilfe dieser Reaktion erreicht werden können (siehe Kapitel 1.2). Die unvorteilhafte Bildung eines ArylRadikals ließ sich in der Gasphase zwar aufgrund der hohen Reaktivität von $\mathrm{Ph}_{2} \mathrm{Fe}(\mathrm{I})^{-}$ gegenüber $\mathrm{Phl}$ in einem solvensfreien Raum bewerkstelligen. Dass dieser Reaktionsprozess so auch in Lösung stattfindet, wo Solvenseffekte und andere Einflüsse eine wesentliche Rolle spielen, darf aber stark bezweifelt werden.

Die Bildung von $\mathrm{Ph}_{3} \mathrm{Fe}(\mathrm{III}) \mathrm{R}^{\prime-}$ konnte in zahlreichen Experimenten erfolgreich nachgewiesen werden, nachdem Phenylferrat-Lösungen direkt mit R'X versetzt wurden. So ist nach der schrittweise ablaufenden oxidativen Addition nur eine weitere Transmetallierung nötig, um das Schlüsselintermediat $\mathrm{Ph}_{3} \mathrm{Fe}(\mathrm{III}) \mathrm{R}^{-}$zu generieren. Mittels unimolekularer Gasphasenexperimente konnte eindeutig nachgewiesen werden, dass heteroleptische Phenylferrate mit Eisen(III)-Kern zur reduktiven Eliminierung tendieren und das Kreuzkupplungsprodukt in Abhängigkeit von sterischen und elektronischen Effekten bilden können. Somit war ein direkter Zugang zum entscheidenden Elementarschritt des Katalyseprozesses möglich, in dessen Zusammenhang mit $\mathrm{Ph}_{2} \mathrm{Fe}(\mathrm{I})^{-}$die katalytisch aktive Spezies zurückgebildet wird. Zusammenfassend ergibt sich ein vollständig über Phenylferrat-Spezies ablaufender 
Katalysezyklus, der auf eine plausible Art und Weise die Bildung des Kreuzkupplungsproduktes aufzeigt ( $\rightarrow$ Schema 35 ).

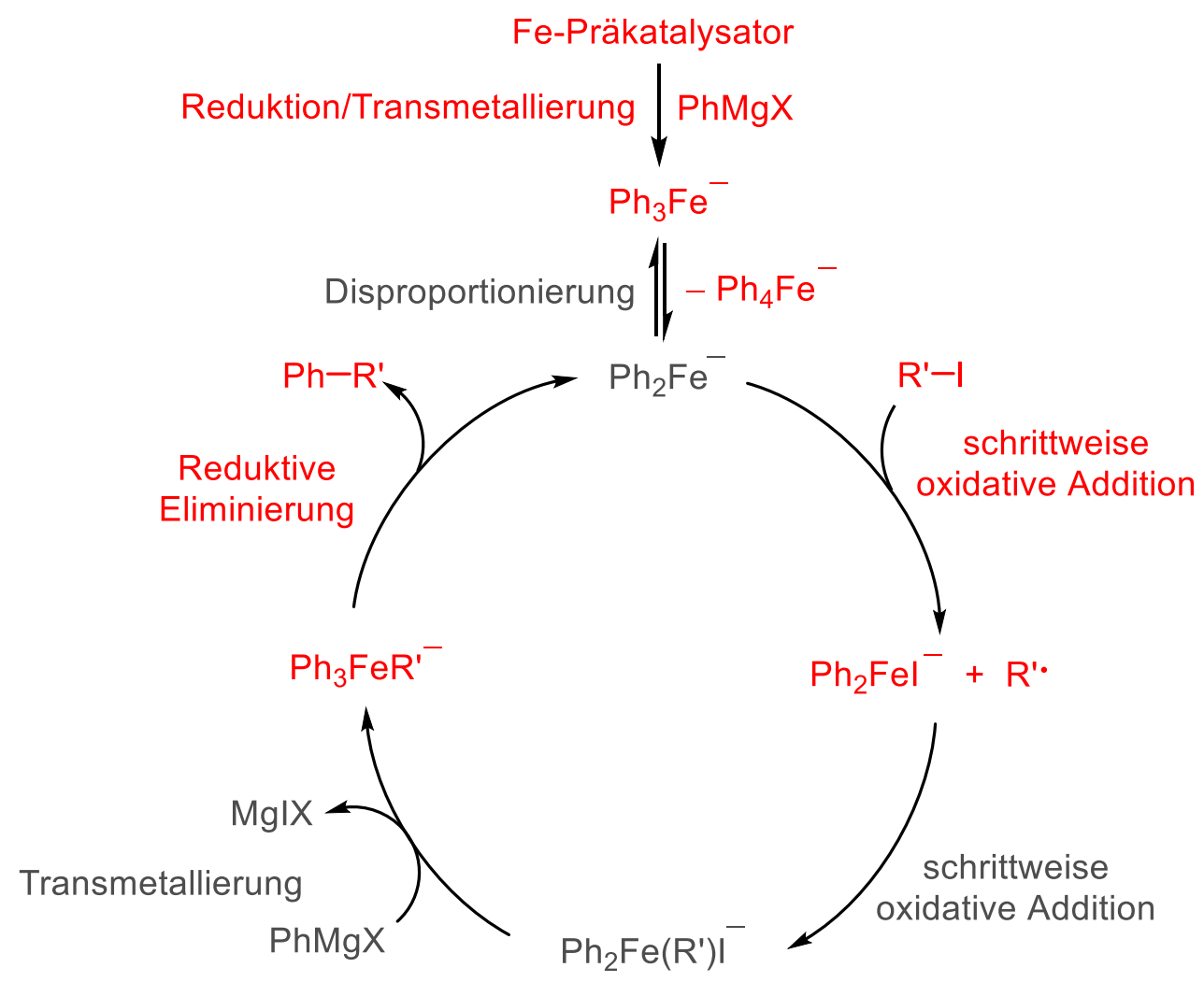

Schema 35. Katalysezyklus für die Eisen-katalysierte Kreuzkupplung von PhMgX mit einem Organyliodid R'I. In rot dargestellte Spezies und Reaktionsschritte konnten direkt nachgewiesen werden, wohingegen für die grau hervorgehobenen Verbindungen und Reaktionsprozesse nur indirekte Hinweise auf deren Präsenz und Stattfinden vorlagen. Durch Reduktion und Transmetallierung eines Eisen-Präkatalysators entsteht das Phenylferrat $\mathrm{Ph}_{3} \mathrm{Fe}(\mathrm{II})^{-}$, das über Disproportionierung $\mathrm{Ph}_{4} \mathrm{Fe}(\mathrm{III})^{-}$und $\mathrm{Ph}_{2} \mathrm{Fe}\left(\mathrm{I}^{-}\right.$bildet. Letztere reagiert mit $\mathrm{R}^{\prime} \mathrm{l}$ über eine schrittweise, oxidative Addition. Nach anschließender Transmetallierung entsteht der heteroleptische Komplex $\mathrm{Ph}_{3} \mathrm{Fe}(\mathrm{III}) \mathrm{R}^{\prime}$, der über reduktive Eliminierung das Kreuzkupplungsprodukt freisetzt und $\mathrm{Ph}_{2} \mathrm{Fe}\left(\mathrm{l}^{-}\right)^{-}$regeneriert.

Für andere Transmetallierungssysteme muss die entsprechende Kreuzkupplung nicht zwingend auf identische Weise ablaufen. So zeigten Fürstner und Mitarbeiter, dass Systeme, die $\beta$-H-Atome aufweisen, sich deutlich in ihrem Reaktionsverhalten unterscheiden können. ${ }^{[35 b]}$ Dieselbe Forschungsgruppe stellte zudem die These auf, dass verschiedenste Eisen-Spezies katalytische Aktivität aufweisen und als Folge mehr als ein Katalysezyklus im zugrunde liegenden Reaktionsmechanismus präsent sein kann. Die in dieser Arbeit gewonnenen Erkenntnisse zeigen, dass für $\mathrm{BnMgCl}$ und $\mathrm{BuMgCl}$ ein ähnlicher Mechanismus eine Rolle spielen kann. So sind in diesen Fällen die Spezies $\mathrm{R}_{3} \mathrm{Fe}(\mathrm{III}) \mathrm{R}^{--}$und $\mathrm{R}_{2} \mathrm{Fe}(\mathrm{II}) \mathrm{X}^{-}$direkt aus den Reaktionslösungen nachweisbar. Im Falle von $\mathrm{R}=\mathrm{Bu}$ ließ sich zudem mit $\mathrm{Bu}_{2} \mathrm{Fe}^{-}$eine 
hochsensitive Eisen(I)-Spezies detektieren. Alle diese Organoferrate sind charakteristisch für den oben beschriebenen Katalysezyklus und geben Hinweise auf einen analog ablaufenden Katalysezyklus für diese Systeme. Dass in diesen Fällen weitere und/oder andere Reaktionsmechanismen eine Rolle spielen, kann zum jetzigen Zeitpunkt aber nicht ausgeschlossen werden.

Mit den Mesitylferraten wurde ein weiteres aromatisches Organoferrat-System untersucht. In der Literatur ist einzig die Kreuzkupplung von MesMgBr mit OctBr bekannt, die nach langen Reaktionszeiten (18 h) nur schwache Ausbeuten an Kreuzkupplungsprodukt liefert. ${ }^{[39 e, 82]}$ Diese für Eisen-katalysierte Kreuzkupplungen untypische Reaktionszeit belegt erneut, dass Eisen(II)-At-Komplexe wenig katalytisch aktiv sind, vor allem dann, wenn sterisch große Substituenten an diesen gebunden vorliegen. Der sterische Anspruch ist auch der Grund dafür, weshalb $\mathrm{Mes}_{3} \mathrm{Fe}(\mathrm{II})^{-}$nicht (oder nur sehr langsam) disproportionieren kann.

Ein gänzlich anderes Verhalten ist für die Transmetallierung mit MeMgX mit anschließenden Folgereaktionen $\mathrm{zu}$ beobachten. Auch wenn mit $\mathrm{Me}_{12} \mathrm{Fe}_{8}{ }^{-}$ein literaturbekanntes Organoferrat ${ }^{[39 g]}$ direkt nachgewiesen werden konnte, deuten alle Experimente darauf hin, dass At-Komplexe hier nur eine untergeordnete Rolle spielen. Höherkernige Aggregate zeigten in den Experimenten wenig Reaktivität gegenüber Organylhalogeniden. Zudem zeigt die Literatur, dass speziell $\mathrm{Me}_{12} \mathrm{Fe}_{8}{ }^{-}$nur dann reaktiv in Kreuzkupplungsreaktionen ist, wenn zusätzliche Äquivalente an MeMgX vorhanden sind. ${ }^{[39 g]}$ Dies legt die Vermutung nahe, dass der achtkernige Eisen-Komplex in diesem speziellen Fall eher als ein Reservoir für die eigentlich katalytisch aktive, bisher unbekannte Eisen-Spezies verstanden werden muss. 


\section{Schlussfolgerung \& Ausblick}

Mechanistische Studien sind von immenser Bedeutung, um eine Synthesemethode zu optimieren und damit auf Dauer zu etablieren. Im Rahmen dieser Arbeit wurde insbesondere ESI-Massenspektrometrie als Analysetechnik zur mechanistischen Aufklärung Eisenkatalysierter Kumada-Kreuzkupplungsreaktionen eingesetzt. So war es erstmals möglich, Organoferrate als mögliche Intermediate dieser Reaktionsklasse in einem Umfang nachzuweisen und zu beschreiben, wie er bisher in der Literatur nicht zu finden war. Als weitere Analysemethoden wurden Mößbauer-Spektroskopie und elektrische Konduktometrie verwendet. Diese konnten nicht nur die aus den ESI-massenspektrometrischen Analysen gewonnen Resultate bestätigen, sondern auch neue Erkenntnisse liefern, mit denen sich die Organoferrate noch detaillierter charakterisieren ließen.

Es konnte gezeigt werden, dass die Reduktion und Transmetallierung gängiger Eisenvorläufer primär zur Bildung von Organoferraten führte, wohingegen neutrale Organoeisen-Spezies unter ligandfreien Bedingungen nur eine untergeordnete Rolle zu spielen scheinen. Anionen der allgemeinen Form $\mathrm{R}_{3} \mathrm{Fe}(\mathrm{II})^{-}$und $\mathrm{R}_{4} \mathrm{Fe}(\mathrm{III})^{-}$waren dominierend, wobei ein low-Spin-Zustand für die Eisen(II)- und ein high-spin-Zustand für die Eisen(III)-Spezies anzunehmen waren. Die Bildung von $\mathrm{R}_{4} \mathrm{Fe}(\mathrm{III})^{-}$lässt sich durch eine Disproportionierung der Eisen(II)-Spezies unter reduktiven Bedingungen erklären. Diese Redoxreaktion war dabei stark vom sterischen Anspruch der organischen Substituenten abhängig. Sterisch anspruchsvolle Systeme lagen primär als $\mathrm{R}_{3} \mathrm{Fe}(\mathrm{II})^{-}$vor, wohingegen für Systeme mit kleinen organischen Resten die Eisen(III)-Spezies dominierten. Gleichzeitig induzierte der geringe sterische Anspruch eine höhere Tendenz zur Aggregation, wodurch auch mehrkernige Spezies wie $\mathrm{Me}_{12} \mathrm{Fe}_{8}{ }^{-}$gebildet wurden. Komplexe des Typs $\mathrm{R}_{2} \mathrm{Fe}(\mathrm{I})^{-}$, die im Zuge der Disproportionierungsreaktion entstanden sein mussten, konnten aufgrund ihrer Labilität und Sensitivität gegenüber Überreduktionsprozessen nur in einem Fall detektiert werden. Für Feststoffe, die während der Transmetallierung ausfielen, konnte Eisen in einer durchschnittlichen Oxidationsstufe von 0.5 nachgewiesen werden, was die Präsenz reduzierter Eisen-Verbindungen bestätigte. Der Feststoff zeigte sich gegenüber organischen Substraten nicht reaktiv. Nichtsdestotrotz belegte seine Entstehung, dass in Eisen-katalysierten Kreuzkupplungsreaktionen eisenhaltige Feststoffe stets berücksichtigt werden sollten.

Durch Einsatz monodentater und bidentater Phosphanliganden war es möglich, niedervalente Eisen-Spezies zu stabilisieren und damit nachweisbar zu machen. Hingegen konnte für TMEDA als Additiv nur ein indirekter Einfluss auf die Organoferrate durch die Ausbildung neuer Gegenkationen ausgemacht werden. Diese sorgten für eine generell geringere 
Aggregationstendenz und stabilisierten insbesondere $\mathrm{R}_{3} \mathrm{Fe}(\mathrm{II})^{-}$. Zum Vergleich erfolgten Transmetallierungsexperimente auch mit $\mathrm{RLi}$ und $\mathrm{R}_{2} \mathrm{Zn}$. Im Falle der OrganolithiumReagenzien konnten vergleichbare Resultate generiert werden, wohingegen die Transmetallierung mit Organozink-Reagenzien aufgrund ihrer geringeren Reaktivität primär zur Ausbildung neutraler, nicht nachweisbarer Eisen-Spezies führte. Im letztgenannten Fall war es möglich, durch den Einsatz bidentater Phosphanliganden Eisen-Spezies als Kationen sichtbar zu machen.

Um die Eisen-katalysierte Kreuzkupplung im Detail zu verstehen, ist es nicht nur von entscheidender Bedeutung, nachzuweisen, welche Oxidationsstufen die während dieser Reaktion entstehenden Intermediate aufweisen. Es ist auch von enormer Wichtigkeit, zu zeigen, dass die entstehenden Spezies in Kreuzkupplungsprozessen reaktiv sind. Deshalb wurde neben der Transmetallierung die Interaktion der Organoferrate gegenüber organischen Substraten R'X intensiv untersucht. In Lösung bildeten sich zumeist heteroleptische Komplexe des Typs $\mathrm{R}_{3} \mathrm{FeR}^{\prime-}$ und, in seltenen Fällen, auch Spezies der Form $\mathrm{R}_{2} \mathrm{FeX}^{-}$als Additionsprodukte. Eine Apparatur wurde im Rahmen dieser Arbeit entwickelt, um die bimolekulare Gasphasen-Reaktivität ausgewählter Organoferrate gegenüber organischen Substraten zu untersuchen. So konnte gezeigt werden, dass $\mathrm{Ph}_{2} \mathrm{Fe}(\mathrm{I})^{-}$in einer Reaktion mit R'I Spezies der Form $\mathrm{Ph}_{2} \mathrm{Fel}^{-}$generiert. Eine schrittweise auftretende oxidative Addition des organischen Substrates würde die Bildung sämtlicher Additionsprodukte in Lösung und in der Gasphase erklären.

Fragmentierungsexperimente in der Gasphase wurden genutzt, um die intrinsische Reaktivität typischer Organoferrate im Detail zu untersuchen. Die reduktive Eliminierung war vor allem für arylhaltige Organoferrate mit Eisen(III)-Kern präsent und lieferte einen direkten Beweis für den entscheidenden Reaktionsschritt im Katalysezyklus, der in der Entstehung des Kreuzkupplungsproduktes mündete. Diese Reaktion zeigte sich stark abhängig von elektronischen und sterischen Effekten der organischen Reste. Zudem konnte eine Vielzahl an Konkurrenzreaktionen beobachtet werden. Neben Zwei-Elektronen-Reaktionen spielten auch radikalische Prozesse eine wichtige Rolle und zeigten die Komplexität der OrganoeisenChemie auf.

Die Resultate, die im Zuge dieser Arbeit gesammelt wurden, konnten in ihrer Gesamtheit zur Entwicklung eines alternativen Katalysezyklus für die Eisen-katalysierte KumadaKreuzkupplung von PhMgX mit Organylhalogeniden genutzt werden, der ausschließlich auf anionischen Eisen-Spezies als Reaktionsintermediaten beruhte. Dieser kann als Grundlage genutzt werden, um weitere Eisen-Kreuzkupplungssysteme und deren mechanistische 
Hintergründe aufzudecken. Diese sind von entscheidender Bedeutung, um Eisen-katalysierte Kreuzkupplungsreaktionen an sich in Zukunft synthetisch effizienter zu gestalten, praktikabler zu machen und eine Möglichkeit zu schaffen, synthetisch nutzbare Voraussagen zu treffen. So demonstriert diese Arbeit, dass unter ligandfreien Bedingungen der Eisen-Präkatalysator nur wenig Einfluss auf die Reaktion hat. Damit ist es nicht von primärer Bedeutung, einfache Eisenvorläufer auf ihre katalytische Relevanz hin synthetisch zu untersuchen. Im Gegensatz dazu kann die Anwesenheit eines Liganden dazu führen, dass auch die Anionen des EisenPräkatalysators die katalytische Aktivität beeinflussen können. Auch die Wahl des Liganden hat einen großen Einfluss auf die Stabilität niedervalenter Eisen-Spezies. Speziell Liganden, die zu $\pi$-Rückbindungen in der Lage sind, können helfen, Eisen(I)- und andere elektronenreiche, katalytisch relevante Eisen-Verbindungen zu stabilisieren, ungewollte Nebenreaktionen wie Überreduktionen einzudämmen und damit effektive Katalysatoren für die Eisen-katalysierten Kumada-Kreuzkupplungsreaktionen zu schaffen. Bedenkt man zusätzlich die elektronischen und sterischen Einflüsse auf die reduktive Eliminierung, kann auch die Wahl des Grignard-Reagenzes und des organischen Substrates entscheidend für die Effektivität der Reaktion sein. Ist der organische Rest am Grignard-Reagenz elektronenreicher und sterisch anspruchsvoller als der organische Rest des Substrates, sollte dies die reduktive Eliminierung des Kreuzkupplungsproduktes ausgehend vom heteroleptischen Eisen(III)Schlüsselintermediat favorisieren.

Für die Zukunft sind weitere mechanistische Studien erforderlich, um ein noch breiteres Verständnis über Organoferrat- sowie Organoeisenchemie und damit über die Eisenkatalysierte Kreuzkupplungsreaktion an sich zu erlangen. Insbesondere die Untersuchung der bimolekularen Gasphasenreaktivität, die durch die im Zuge dieser Arbeit entwickelte Apparatur in Kombination mit ESI-Quadrupol-Ionenfallen-Massenspektrometrie erfolgte, ermöglicht eine Vielzahl an weiteren Experimenten und Analysemöglichkeiten. Bisher wurde nur die Reaktivität der Phenylferrate gegenüber einfachen organischen Substraten untersucht. Weitere Organoferrate sollten im Rahmen solcher Experimente analysiert werden, um Gemeinsamkeiten und Unterschiede zu der Reaktivität der Phenylferrate aufzuzeigen. Zudem lassen sich mit dieser Analysetechnik Oxidations- und Hydrolysereaktionen unter kontrollierten und damit reproduzierbaren Bedingungen untersuchen, indem definierte Mengen an Sauerstoff oder Wasser in die Gasphase eingebracht werden. Kenntnisse über diese Reaktionen können wichtige Informationen darüber liefern, inwieweit diese zumeist ungewollten Nebenreaktionen sich auf die Organoferrate auswirken können. Auch jenseits der Organoferrat-Chemie bietet die Untersuchung der bimolekularen Gasphasenreaktivität eine ideale Möglichkeit, um Additionsprozesse an Metallintermediate besser verstehen zu können. 
Neben ihrer Teilnahme an Kreuzkupplungsreaktionen können Organoferrate auch an anderen Reaktionsprozessen beteiligt sein. So ist es denkbar, dass die anionischen Eisen-Spezies auch Teil des Mechanismus Eisen-katalysierter $\mathrm{CH}$-Aktivierungen sind, da auch hier angenommen wird, dass über Transmetallierung eines Eisen-Präkatalysators die eigentlich katalytisch aktive Organoeisen-Spezies generiert wird. ${ }^{[103]}$ Dies bedarf einer genaueren Untersuchung der Reaktivität der Organoferrate gegenüber organischen Substraten, die typischerweise in $\mathrm{CH}$-Aktivierungsreaktionen eingesetzt werden. Zudem ist es möglich, über eine genaue Kenntnis der Organoferrat-Chemie gänzlich neue Eisen-katalysierte Reaktionen zu entwickeln, in denen die Reaktivität dieser Spezies gezielt ausgenutzt wird.

Erfolgreich durchgeführte Synthesen auf dem Gebiet der Eisen-katalysierten Kreuzkupplungsreaktionen zeigen, dass neben den Grignard-Reagenzien eine Vielzahl weiterer Nukleophile in Katalyseprozessen eingesetzt werden können (siehe Kapitel 1.2). So sollten mechanistische Studien auf alternative Transmetallierungsreagenzien ausgeweitet werden, um auch hier für ein breiteres mechanistisches Verständnis zu sorgen.

Ganz allgemein können die bisherigen sowie zukünftigen Ergebnisse noch durch theoretische Rechnungen und weitere Analysetechniken gestützt und erweitert werden. So sind neben Röntgen-Kristallstrukturanalysen vor allem auch weitere Methoden wie ESR- und UV/VisSpektroskopie denkbar. Die beiden letztgenannten Techniken mögen allein zwar nur schwer zu interpretierende Messdaten liefern, können aber in Kombination mit den Ergebnissen aus dieser Arbeit neue, wertvolle Hinweise liefern und damit zusätzlich zum Verständnis Eisenkatalysierter Kreuzkupplungsreaktionen beitragen. 


\section{Experimentalteil}

Alle Ergebnisse, die im Rahmen dieser Arbeit präsentiert und diskutiert werden, wurden jeweils durch mindestens eine Reproduktionsmessung bestätigt.

\subsection{Materialien und Methoden}

\subsubsection{Allgemeine Arbeitstechniken und Methoden}

Alle Arbeiten mit luft- und feuchtigkeitsempfindlichen Substanzen wurden mittels SchlenkTechnik in trockener Argonatmosphäre durchgeführt. ${ }^{[104]}$ THF wurde über Kaliumhydroxid vorgetrocknet, über Natrium und Benzophenon getrocknet und vor Gebrauch frisch destilliert. TMEDA und $\mathrm{Et}_{2} \mathrm{O}$ wurden über Calciumhydrid vorgetrocknet, über Natrium getrocknet und destilliert. NMR-Experimente wurden an einem Avance III 300-Spektrometer von Bruker Daltonik (300 MHz) durchgeführt.

\subsubsection{Chemikalien}

Alle verwendeten Chemikalien wurden von abcr GmbH (Karlsruhe, Deutschland), Acros Organics/Thermo Fisher Scientific (Geel, Belgien), Carl Roth GmbH (Karlsruhe, Deutschland), Merck (Darmstadt, Deutschland) oder Sigma-Aldrich (Taufkirchen, Deutschland) käuflich erworben. ${ }^{57} \mathrm{Fe}-$ Pulver wurde in 95\%iger Anreicherung von Isoflex (San Fransisco, USA) bezogen.

\subsubsection{Software}

Zur Auswertung und Darstellung der Massenspektren wurde das Programm Data Analysis 4.2 von Bruker Daltonik verwendet. Isotopenmuster inkl. der exakten Massen wurden mit Hilfe des Programms Compass Isotope Pattern 2.0 von Bruker Daltonik berechnet. ESI-Massenspektren, Isotopenmuster, kinetische Daten sowie Mößbauer-Spektren ${ }^{\text {iv }}$ wurden mit OriginPro 8.5 OG SR1 von OriginLab Corporation grafisch aufgearbeitet. MestReNova 10.0.2 von MestReLab Research S. L. diente zur Auswertung und grafischen Darstellung von NMR-Daten. Strukturformeln, Reaktionsgleichungen sowie Reaktions-mechanismen wurden mittels ChemBioDraw Ultra 13 von PerkinElmer erstellt.

\footnotetext{
iv Ich danke Dr. S. Demeshko für die Simulation experimenteller Mößbauer-Daten; E. Bill, Mfit-Programm, MaxPlanck Institut für chemische Energiekonversion, Mühlheim/Ruhr, Deutschland, 2008.
} 


\subsubsection{Komplexometrische Bestimmung von Eisen ${ }^{[105]}$}

Unter Schutzgasatmosphäre wurden eisenhaltige Proben durch Zugabe von Salpetersäure (konz., $5 \mathrm{~mL}$ ) in dieser gelöst und dabei gezielt zu Eisen(III) oxidiert. Anschließend wurden die Probenlösungen mit $p$-Chloranilin auf einen $\mathrm{pH}$-Wert von 2-3 eingestellt und mit Wasser auf ein Gesamtvolumen von $V=25 \mathrm{~mL}$ gebracht. Abschließende komplexometrische Titration erfolgte durch Zugabe von EDTA (0.1 M) mit 5-Sulfosalicylsäure als Indikator (1 mM, $0.5 \mathrm{~mL})$, wobei sich die ursprünglich violette Probenlösung gelb färbte.

\subsubsection{Konzentrationsbestimmung metallorganischer Reagenzien}

Grignard-Reagenzien und zinkorganische Reagenzien. ${ }^{[106]}$ Unter Schutzgasatmosphäre wurde lod (0.1-0.3 mmol) in einer Lösung von $\mathrm{LiCl}$ in THF $(1.5 \mathrm{~mL}, 0.5 \mathrm{M})$ gelöst. Die entstandene rot-braune Lösung wurde bei $0^{\circ} \mathrm{C}$ tropfenweise mit dem entsprechenden metallorganischen Reagenz versetzt, bis ein vollständiges Entfärben der Lösung zu beobachten war. Das eingesetzte Volumen des metallorganischen Reagenz entsprach dabei genau einem Äquivalent in Relation zu der eingesetzten Menge an lod.

Organolithium-Reagenzien. ${ }^{[107]}$ Unter Schutzgasatmosphäre wurde eine Lösung von 1,3-Diphenyl-2-propanon-tosylhydrazon $(0.5 \mathrm{mmol})$ in THF $(5 \mathrm{~mL})$ angesetzt und diese bei $0{ }^{\circ} \mathrm{C}$ nun solange tropfenweise mit dem entsprechenden Organolithium-Reagenz versetzt, bis ein Farbumschlag von farblos zu gelb zu beobachten war. Das eingesetzte Volumen des Organolithium-Reagenz entsprach dabei genau einem Äquivalent in Relation zu der eingesetzten Menge an 1,3-Diphenyl-2-propanon-tosylhydrazon. 


\subsection{Synthesen und Probenvorbereitung}

\subsubsection{Synthese metallorganischer Reagenzien}

Synthese von Grignard-Reagenzien. ${ }^{[55,64 c, 108]}$ Unter Schutzgasatmosphäre wurde Magnesium ( $0.7 \mathrm{~g}, 28 \mathrm{mmol}, 1.1 \mathrm{Äq}$ ) in THF (20 mL) vorgelegt und das jeweilige organische Substrat (25 mmol, 1.0 Äq.) zu einem Viertel hinzugegeben. Nach Start der Reaktion wurde der Rest des organischen Substrates langsam hinzugetropft und die entstandene Lösung über Nacht gerührt. Nach dieser Vorschrift konnten nachfolgende Grignard-Reagenzien erfolgreich synthetisiert werden:<smiles>COc1ccc(N(Br)Br)cc1</smiles><smiles>CN(C)c1ccc(N(Br)Br)cc1</smiles>

$0.8 \mathrm{M}$<smiles>Cl[Mg]Cc1ccccc1</smiles>

\section{$0.5 \mathrm{M}$}<smiles>CCCCC(C)C(C)CCl</smiles>

$1.0 \mathrm{M}$<smiles></smiles>

$0.6 \mathrm{M}$<smiles>CC(C)(C)CCC(C)(C)CCl</smiles>

$1.0 \mathrm{M}$<smiles>Cc1cc(C)c(N)c(Br)c1</smiles>

$1.0 \mathrm{M}$<smiles>CCCCC(C)(C)C(C)CCl</smiles>

$1.0 \mathrm{M}$<smiles></smiles>

$0.9 \mathrm{M}$<smiles>C[Si](C)(C)C[Si]Cl</smiles>

$0.8 \mathrm{M}$

Aromatische Grignard-Reagenzien mit stark elektronenziehenden Substituenten in p-Position wurden über eine alternative Synthesemethode dargestellt. Dabei wurde das entsprechende Organyliodid (5 mmol, 1.0 Äq.) unter Schutzgasatmosphäre bei $-40^{\circ} \mathrm{C}$ in THF vorgelegt und 'PrMgBr (1.0 Äq.) über $1 \mathrm{~h}$ langsam hinzugetropft.<smiles></smiles>

$0.5 \mathrm{M}$<smiles></smiles>

$0.5 \mathrm{M}$ 
Synthese von Mesityllithium. ${ }^{[109]}$ Unter Schutzgas wurde zu einer Lösung von Mesitylbromid (3.8 mL, $25 \mathrm{mmol}, 1.0$ Äq.) in THF (10 mL) unter Rühren bei $-78^{\circ} \mathrm{C}$ vorsichtig Butyllithium (28 mL, $0.9 \mathrm{M}$ in Hexan, 1.0 Äq.) hinzugegeben. Nach weiterem Rühren ( $2 \mathrm{~h}$ ) bei $-78^{\circ} \mathrm{C}$ entstand ein leicht gelber Feststoff, dessen Überstand mittels einer Spritze abgezogen wurde. Waschen mit THF ( $3 \cdot 10 \mathrm{~mL}$ ) und vorsichtiges Abziehen des Überstandes mittels einer Spritze lieferte die Zielverbindung in Form eines farblosen Feststoffes. Das Produkt ist als Feststoff bei $-78^{\circ} \mathrm{C}$ nur wenige Stunden stabil, kann aber als Lösung $(0.14 \mathrm{M}$ in $40 \mathrm{~mL}$ trockenem $\mathrm{Et}_{2} \mathrm{O}$ ) über $2-3 \mathrm{~d}$ bei $0^{\circ} \mathrm{C}$ gelagert und verwendet werden.

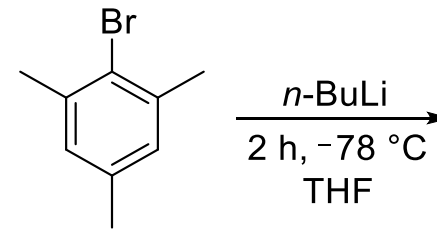

198.0<smiles>Cc1cc(C)c(Cl)c(C)c1</smiles>

126.1

\subsubsection{Eisen-katalysierte Kreuzkupplung von $\mathrm{PhMgCl}$ und ${ }^{i} \mathrm{PrCl}$}

Unter Schutzgas wurde zu einer auf $0^{\circ} \mathrm{C}$ gekühlten Lösung von $\mathrm{Fe}(\mathrm{acac})_{3}(5 \mathrm{~mol} \%)$ und ${ }^{i} \mathrm{PrCl}$ (10 mmol, 1.0 Äq.) in THF (10 mL) über einen Zeitraum von $45 \mathrm{~min} \mathrm{PhMgCl}(2.0 \mathrm{M}, 1.3 \mathrm{mmol}$, 1.3 Äq.) langsam zugegeben. Die entstandene Reaktionsmischung wurde für $24 \mathrm{~h}$ bei Umgebungstemperatur gerührt und $\mathrm{mit}^{\mathrm{Et}} \mathrm{Et}_{2} \mathrm{O}(3 \cdot 20 \mathrm{~mL})$ extrahiert. Trocknen über Natriumsulfat und Entfernen des Lösungsmittels im Vakuum lieferte eine Mischung aus Cumol (6.7 mmol, $67 \%$ ) und Biphenyl (1.1 mmol, $11 \%$ ) als gelbes Öl.

Cumol: ${ }^{1} \mathrm{H}-\mathrm{NMR}\left(300 \mathrm{MHz}, \mathrm{CDCl}_{3}\right): \delta(\mathrm{ppm})=1.18\left(\mathrm{~d}, J=6.9 \mathrm{~Hz}, 6 \mathrm{H}, \mathrm{CH}_{3}\right), 2.83$ (hep, $J=6.9 \mathrm{~Hz}, 1 \mathrm{H}, \mathrm{CH}), 7.15\left(\mathrm{~m}, 5 \mathrm{H}, \mathrm{C}_{\text {arom }}-\mathrm{H}\right)$.

Biphenyl: ${ }^{1} \mathrm{H}-\mathrm{NMR}\left(300 \mathrm{MHz}, \mathrm{CDCl}_{3}\right): \delta(\mathrm{ppm})=7.15\left(\mathrm{~m}, 2 \mathrm{H}, \mathrm{C}_{\text {para }}-\mathrm{H}\right), 7.36(\mathrm{dd}, \mathrm{J}=7.3 \mathrm{~Hz}$, $\left.4 \mathrm{H}, \mathrm{C}_{\text {meta }}-\mathrm{H}\right), 7.51$ (d, J = 7.3 Hz, $\left.4 \mathrm{H}, \mathrm{C}_{\text {ortho }}-\mathrm{H}\right)$. 


\subsubsection{Weitere Synthesen}

\section{Synthese von $57-$ Eisen(II)chlorid $\left({ }^{57} \mathrm{FeCl}_{2}\right)^{[74]}$}

$$
\begin{aligned}
& \text { 1. } \mathrm{rt}, 5 \mathrm{~h} \\
& { }^{57} \mathrm{Fe}+2 \mathrm{HCl} \underset{\mathrm{MeOH}}{\stackrel{2 . \Delta, 5 \mathrm{~h}}{\longrightarrow}}{ }^{57} \mathrm{FeCl}_{2}+\mathrm{H}_{2} \\
& \begin{array}{llll}
56.9 & 36.0 & 127.9 & 2.0
\end{array}
\end{aligned}
$$

Unter Schlenkbedingungen wurde zu einer Suspension von 57-Eisenpulver (250 mg, $4.40 \mathrm{mmol}$, $1.00 \mathrm{Äq}$ ) in $\mathrm{MeOH}\left(5 \mathrm{~mL}\right.$ ) langsam $\mathrm{HCl}$ (konz. in $\mathrm{H}_{2} \mathrm{O}, 0.8 \mathrm{~mL}, 2.0$ Äq.) hinzugetropft und das Lösungsmittel nach vollendeter $\mathrm{H}_{2}$-Entwicklung im Vakuum entfernt. Trocknen im Vakuum bei $150{ }^{\circ} \mathrm{C}$ lieferte die Zielverbindung (470 mg, $\left.3.67 \mathrm{mmol}, 83 \%\right)$ als beigen Feststoff.

MS $\left(\mathrm{ESI}^{+}, \mathrm{THF}\right): m / z=\quad 308.6 \quad\left[{ }^{57} \mathrm{FeCl}(\mathrm{THF})_{3}\right]^{+}, \quad 435.3 \quad\left[{ }^{57} \mathrm{Fe}_{2} \mathrm{Cl}_{3}(\mathrm{THF})_{3}\right]^{+}, \quad 564.0$ $\left[{ }^{57} \mathrm{Fe}_{3} \mathrm{Cl}_{5}(\mathrm{THF})_{3}\right]^{+}, 690.7\left[{ }^{57} \mathrm{Fe}_{4} \mathrm{Cl}_{7}(\mathrm{THF})_{3}\right]^{+}$.

MS $\left(\mathrm{ESI}^{-}, \mathrm{THF}\right): m / z=162.0\left[{ }^{57} \mathrm{FeCl}_{3}\right]^{-}, 199.0\left[{ }^{57} \mathrm{FeCl}_{4}\right]^{-}, 290.9\left[{ }^{57} \mathrm{Fe}_{2} \mathrm{Cl}_{5}\right]^{-}, 325.9\left[{ }^{57} \mathrm{Fe}_{2} \mathrm{Cl}_{6}\right]^{-}$, $417.7\left[{ }^{57} \mathrm{Fe}_{3} \mathrm{Cl}_{7}\right]^{-}, 544.4\left[{ }^{57} \mathrm{Fe}_{4} \mathrm{Cl}_{9}\right]^{-}, 693.1\left[{ }^{57} \mathrm{Fe}_{5} \mathrm{Cl}_{11}\right]^{-}$.

\section{Synthese von (4-Brombut-2-en-1-yl)triphenylphosphoniumbromid ${ }^{[110]}$}

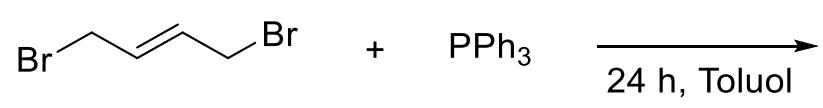

213.9<smiles>BrC/C=C/C[Pb]Br</smiles>

476.0

Zu einer Lösung von 1,4-Dibrom-but-2-en (8.6 g, $40 \mathrm{mmol}, 1.0$ Äq.) in Toluol wurde eine Lösung von Triphenylphosphan (11 g, 40 mmol, 1.0 Äq.) in Toluol hinzugegeben und die entstandene Reaktionsmischung für $24 \mathrm{~h}$ bei Umgebungstemperatur gerührt. Der dabei entstandene Feststoff wurde abfiltriert und mit Toluol $(4.5 \mathrm{~mL})$ gewaschen. Trocknung im Vakuum lieferte die Zielverbindung (15 g, 32 mmol, 80 \%) als farblosen Feststoff.

${ }^{1} \mathrm{H}-\mathrm{NMR}\left(300 \mathrm{MHz}, \mathrm{CD}_{3} \mathrm{OD}\right): \delta(\mathrm{ppm})=3.97\left(\mathrm{dd}, J=15.1,3.2 \mathrm{~Hz}, 2 \mathrm{H}, \mathrm{CH}_{2}-\mathrm{P}\right), 4.35$ (dd, $\left.J=15.8,7.5 \mathrm{~Hz}, 2 \mathrm{H}, \mathrm{CH}_{2}-\mathrm{Br}\right), 5.78-5.84(\mathrm{~m}, 1 \mathrm{H}, \mathrm{CH}), 6.02-6.08(\mathrm{~m}, 1 \mathrm{H}, \mathrm{CH})$, 7.74-7.82 (m, $\left.12 \mathrm{H}, \mathrm{C}_{\text {arom }}-\mathrm{H}\right), 7.88-7.94\left(\mathrm{~m}, 3 \mathrm{H}, \mathrm{C}_{\text {arom }}-\mathrm{H}\right)$.

MS (ESI+, THF): $m / z=315.6[\mathrm{M}-2 \mathrm{Br}+\mathrm{H}]^{+}, 395.4[\mathrm{M}-\mathrm{Br}]^{+}$. 


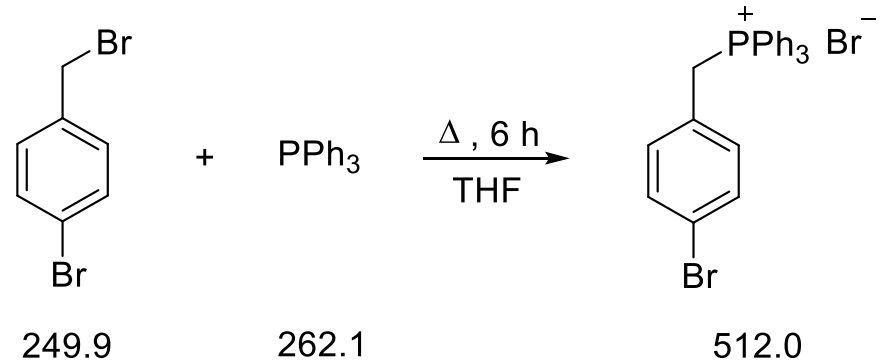

Eine Lösung von Triphenylphosphan (3.0 g, $11 \mathrm{mmol}, 1.1$ Äq.) und 1-Bromo-4-(bromomethyl)benzol (2.5 g, $10 \mathrm{mmol}, 1.0$ Äq.) in THF (50 mL) wurde $6 \mathrm{~h}$ unter Rückfluss erhitzt. Der dabei entstandene Feststoff wurde abfiltriert und $\mathrm{mit}^{\mathrm{Et}_{2} \mathrm{O}}(3 \cdot 10 \mathrm{~mL})$ gewaschen. Trocknung im Vakuum lieferte die Zielverbindung $(4.4 \mathrm{~g}, 8.5 \mathrm{mmol}, 75$ \%) als farblosen Feststoff.

${ }^{1} \mathrm{H}-\mathrm{NMR}\left(300 \mathrm{MHz}, \mathrm{CD}_{3} \mathrm{OD}\right): \delta(\mathrm{ppm})=5.50\left(\mathrm{~d}, J=15.0 \mathrm{~Hz}, 2 \mathrm{H}, \mathrm{CH}_{2}\right), 6.91(\mathrm{~d}, J=6.5 \mathrm{~Hz}$, $\left.2 \mathrm{H}, \mathrm{C}_{\text {arom }}-\mathrm{H}\right), 7.4\left(\mathrm{~d}, J=6.5 \mathrm{~Hz}, 2 \mathrm{H}, \mathrm{C}_{\text {arom }}-\mathrm{H}\right), 7.63-7.77\left(\mathrm{~m}, 12 \mathrm{H}, \mathrm{C}_{\text {arom }}-\mathrm{H}\right), 7.88-7.95(\mathrm{~m}, 3 \mathrm{H}$, $\left.\mathrm{C}_{\text {arom }}-\mathrm{H}\right)$.

MS (ESI $\left.{ }^{+}, \mathrm{THF}\right): m / z=431.4[\mathrm{M}-\mathrm{Br}]^{+}$.

Synthese von 1,3-Diphenyl-2-propanon-tosylhydrazon ${ }^{[107]}$<smiles>Cc1ccc(S(=O)(=O)NN)cc1</smiles>

186.1<smiles>O=C(Cc1ccccc1)Cc1ccccc1</smiles>

210.3<smiles>COC(C)(C)[CH]Cl</smiles>

378.1

Zu einer mit konz. $\mathrm{HCl}(0.2 \mathrm{~mL})$ angesäuerten Lösung von Tosylhydrazin $(1.9 \mathrm{~g}, 10 \mathrm{mmol}$, 1.0 Äq.) in Ethanol (30 mL) wurde unter Rühren bei $50^{\circ} \mathrm{C}$ 1,3-Diphenyl-2-propanon $(2.1 \mathrm{~g}$, $10 \mathrm{mmol}, 1.0$ Äq.) hinzugegeben und die Reaktionsmischung über Nacht bei $0^{\circ} \mathrm{C}$ gelagert. 
Der auskristallisierte Feststoff wurde abfiltriert und mit auf $0^{\circ} \mathrm{C}$ vorgekühltem Ethanol $(3 \cdot 5 \mathrm{~mL})$ gewaschen. Trocknung im Vakuum lieferte die Zielverbindung $(3.1 \mathrm{~g}, 8.2 \mathrm{mmol}$, $82 \%)$ als farblosen Feststoff.

${ }^{1} \mathrm{H}-\mathrm{NMR}\left(300 \mathrm{MHz}, \mathrm{CDCl}_{3}\right): \delta(\mathrm{ppm})=2.50\left(\mathrm{~s}, 3 \mathrm{H}, \mathrm{CH}_{3}\right), 3.43\left(\mathrm{~s}, 2 \mathrm{H}, \mathrm{CH}_{2}\right), 3.54\left(\mathrm{~s}, 2 \mathrm{H}, \mathrm{CH}_{2}\right)$, 7.75-6.81 (m, $\left.2 \mathrm{H}, \mathrm{C}_{\text {arom }}-\mathrm{H}\right), 6.97-7.02\left(\mathrm{~m}, 2 \mathrm{H}, \mathrm{C}_{\text {arom }}-\mathrm{H}\right)$, 7.17-7.33 (m, $\left.8 \mathrm{H}, \mathrm{C}_{\text {arom }}-\mathrm{H}\right), 7.63-7.68$ (m, $\left.2 \mathrm{H}, \mathrm{C}_{\text {arom }}-\mathrm{H}\right)$.

\subsubsection{Synthese und Isolation eisenhaltiger Feststoffe}

Unter Schutzgas-Atmosphäre wurde eine Lösung von $\mathrm{FeCl}_{2}(5 \mathrm{mM})$ und TMEDA (4.0 Äq.) in THF (80 mL) mit MeMgCl (4.0 Äq.) versetzt und die entstandene Reaktionsmischung für 30 min bei Umgebungstemperatur gerührt. Anschließend wurde die Lösung mit einer Spritze abgezogen und der zurückbleibende, eisenhaltige Feststoff für $6 \mathrm{~h}$ im Vakuum getrocknet.

\subsubsection{Probenvorbereitung}

ESI-Massenspektrometrie. Unter Schutzgasatmosphäre wurde ein Eisenvorläufer (10 oder $20 \mathrm{mM}$ ) in THF (4 mL) vorgelegt und das entsprechende Grignard-Reagenz (1-4 Äq.) bei $0^{\circ} \mathrm{C}$ langsam hinzugegeben. Reaktionen mit $\mathrm{EtMgCl}$ oder $\mathrm{BuMgCl}$ wurden analog dazu bei $\quad-78^{\circ} \mathrm{C}$ durchgeführt. Erfolgte die Probenvorbereitung unter Zugabe eines lithiumorganischen- oder zinkorganischen Reagenzes, wurde die Temperatur $\left(-78^{\circ} \mathrm{C} /\right.$ Umgebungstemperatur) entsprechend angepasst. Die Zugabe von Additiven erfolgte unmittelbar vor Zugabe des metallorganischen Reagenzes. Die Zugabe von aliphatischen oder aromatischen Halogeniden sowie von lod, Sauerstoff oder Wasser (je 0.5 Äq.) erfolgte 30 min nach Zugabe des metallorganischen Reagenzes. Reaktionen mit zwei unterschiedlichen Grignard-Reagenzien (je 2 Äq.) wurden durch gleichzeitiges Zugeben beider Reagenzien durchgeführt. Bei Austauschreaktionen wurden die Probenlösungen separat angesetzt und nach 30 min vereint.

Elektrische Leitfähigkeit. Zu einer auf $0^{\circ} \mathrm{C}$ vorgekühlten Lösung eines Eisenvorläufers (50 mM) in THF $(20 \mathrm{~mL})$ wurde unter Schutzgasatmosphäre langsam das entsprechende Grignard-Reagenz (4 Äq.) hinzugegeben und die entstandene Reaktionsmischung für 30 min bei $0^{\circ} \mathrm{C}$ gerührt. Erfolgte die Probenvorbereitung unter Zugabe eines OrganolithiumReagenzes, wurde die Temperatur auf $-78^{\circ} \mathrm{C}$ gesenkt. In Fällen, in denen TMEDA als Additiv zugesetzt wurde, erfolgte dessen Zugabe vor der des metallorganischen Reagenz. Bei einer 
kinetischen Betrachtung der Reaktion der Produkte einer Fe(acac) $3 / 4 \mathrm{PhMgCl}-$ Probenlösung mit oder ohne ${ }^{i} \mathrm{PrCl}$ (0.5 Äq.) in Abwesenheit oder Anwesenheit von TMEDA (4.0 Äq.) wurde das Organylhalogenid direkt in die Probenlösung der Messzelle zugegeben und die Leitfähigkeit im Abstand von $10 \mathrm{~s}$ in einem Zeitraum von 30 min gemessen.

Mößbauer-Spektroskopie. Zu einer Lösung von ${ }^{57} \mathrm{FeCl}_{2}(5 \mathrm{mM})$ in THF (1 mL) wurde unter Schutzgasatmosphäre langsam das entsprechende Grignard-Reagenz (4.0 Äq.) bei Umgebungstemperatur hinzugegeben und die entstandene Reaktionsmischung in die Mößbauermesszelle transferiert. Unmittelbar danach wurde die Messzelle inklusive der Probenlösung in flüssigem Stickstoff eingefroren. Wurden den Reaktionslösungen Additive oder organische Substrate beigefügt, erfolgte deren Zugabe unmittelbar vor bzw. nach der des metallorganischen Reagenz.

Bimolekulare Gasphasenreaktivität. Zur Untersuchung literaturbekannter bimolekularer Substitutionsreaktionen in der Gasphase wurde $\mathrm{CF}_{3} \mathrm{CH}_{2} \mathrm{OH}$ (10 mM) in Methanol (5 mL) gelöst und mit 5 Tropfen $\mathrm{NaOH}$ (in $\mathrm{H}_{2} \mathrm{O}, 1 \mathrm{~mm}$ ) versetzt. Die Gasphasenreaktionen erfolgten gegenüber $\operatorname{EtBr}(V=12 \mu \mathrm{L}, 6 \mu \mathrm{L}, 3 \mu \mathrm{L}, 1.5 \mu \mathrm{L}, 0.75 \mu \mathrm{L})$, ${ }^{i} \operatorname{PrBr}(V=12 \mu \mathrm{L}, 6 \mu \mathrm{L}, 3 \mu \mathrm{L}, 1.5 \mu \mathrm{L}$, $0.75 \mu \mathrm{L}$ ) oder $\mathrm{Mel}(V=8 \mu \mathrm{L}, 4 \mu \mathrm{L}, 2 \mu \mathrm{L}, 1 \mu \mathrm{L}, 0.5 \mu \mathrm{L})$. In weiteren Experimenten wurde $\mathrm{NEt}_{4} \mathrm{Br}$ $(1 \mathrm{mM})$ in Methanol $(5 \mathrm{~mL})$ gelöst. Die Gasphasenreaktionen erfolgten gegenüber Mel $(V=128 \mu \mathrm{L}, 64 \mu \mathrm{L}, 32 \mu \mathrm{L}, 16 \mu \mathrm{L}, 8 \mu \mathrm{L}), \mathrm{CD}_{3} \mathrm{l}(V=128 \mu \mathrm{L}, 64 \mu \mathrm{L}, 32 \mu \mathrm{L}, 16 \mu \mathrm{L}, 8 \mu \mathrm{L})$ oder $\mathrm{CF}_{3} \mathrm{CO}_{2} \mathrm{Me}(\mathrm{V}=1024 \mu \mathrm{L}, 512 \mu \mathrm{L}, 256 \mu \mathrm{L}, 128 \mu \mathrm{L}, 64 \mu \mathrm{L})$. Lösungen der Phenylferrate in Anwesenheit von $\mathrm{PPh}_{3}$ in THF wurden nach dem Standardverfahren für ESI-massenspektrometrische Proben erstellt (siehe oben). Reaktionen erfolgten gegenüber $\operatorname{Mel}(V=10 \mu \mathrm{L}), \operatorname{Etl}(V=30 \mu \mathrm{L}, 20 \mu \mathrm{L}, 10 \mu \mathrm{L}, 5 \mu \mathrm{L}, 2.5 \mu \mathrm{L}),{ }^{i} \operatorname{PrCl}(V=10 \mu \mathrm{L}),{ }^{i} \operatorname{PrBr}(V=10 \mu \mathrm{L})$, ${ }^{i} \operatorname{Prl}(V=40 \mu \mathrm{L}, 30 \mu \mathrm{L}, 20 \mu \mathrm{L}, 10 \mu \mathrm{L}, 5 \mu \mathrm{L})$, ${ }^{t} \mathrm{Bul}(V=40 \mu \mathrm{L}, 30 \mu \mathrm{L}, 20 \mu \mathrm{L}, 10 \mu \mathrm{L}, 5 \mu \mathrm{L})$ oder Phl $(V=10 \mu \mathrm{L})$. Alle neutralen Reaktanten wurden über gasdichte Hamiltonspritzen in den vorgekühlten Transfer-Glaskolben überführt, der unmittelbar danach an die Apparatur angeschlossen wurde. Das weitere Vorgehen erfolgte gemäß Kapitel 4.2.1.1. 


\subsection{Analytische Methoden}

\subsubsection{ESI-Massenspektrometrie}

Der Großteil der Massenspektren wurde mit einer HCT Quadrupol-Ionenfalle (Bruker Daltonik) in einem Massenbereich von $m / z 50-1200$ aufgenommen. Probenlösungen wurden mit einer gasdichten Hamilton-Spritze in die ESI-Quelle durch eine motorbetriebene Spritzenpumpe mit einer Flussrate von $8 \mu \mathrm{L} \mathrm{min}{ }^{-1}$ eingeführt. Die Probe durchlief eine Metallkapillare, an der eine Hochspannung von $3 \mathrm{kV}$ angelegt war. Stickstoff wurde als Zerstäubergas $(0.7$ bar) eingesetzt, um die Bildung des Taylor-Konus zu begünstigen. Zusätzlich wurde Stickstoff als Trockengas $\left(6 \mathrm{~L} \mathrm{~min}^{-1}\right)$ eingesetzt, um ein erleichtertes Verdampfen des Lösungsmittels und das Eintreten von Analyt-Ionen in die Gasphase zu ermöglichen. Die Analyt-lonen wurden bei einer Temperatur von $60^{\circ} \mathrm{C}$ erzeugt, um einen möglichst sanften Übergang in die Gasphase ohne ein hohes $\mathrm{Ma}$ an Fragmentierung zu bewirken. Nach der Kapillare (Spannung am Kapillarenausgang $-20 \mathrm{~V}$ ) durchliefen die Ionen einen Skimmer (-20 V) sowie zwei TransferOktopole $(-4.0 \mathrm{~V} /-1.7 \mathrm{~V})$ und gelangten schließlich in die Quadrupol-lonenfalle. Diese wurde standardmäßig mit einer Trap Drive von 40 betrieben, um die effiziente Detektion von Ionen mit einem mittleren $\mathrm{m} / \mathrm{z}$-Verhältnis zu gewährleisten. Um eine Intensitätszunahme von Peaks mit kleinem oder großem $\mathrm{m} / \mathrm{z}$-Verhältnis zu bewirken, wurde dieser Parameter entsprechend gesenkt oder erhöht. Zwei Linsen zur Fokussierung und zur Erhöhung der Dichte an AnalytIonen wurden mit einer Spannung von $5.0 \mathrm{~V}$ und $60 \mathrm{~V}$ betrieben. Ionen von besonderem Interesse wurden mit einer Isolierungsbreite von $6 \mathrm{u}$ massenselektiert und mit einer Spannungsamplitude $V_{\text {Anr }}$ von $0.1-3.0 \mathrm{~V}$ beschleunigt, um durch Stöße mit Helium $(p \approx 0.5 \mathrm{mTorr})$ unimolekular zu dissoziieren. Typischerweise wurde dabei eine Fragmentierungszeit von $40 \mathrm{~ms}$ bei einem Cut-Off für niedrige $\mathrm{m} / \mathrm{z}$-Verhältnisse von $27 \%$ (in Relation zum isolierten lon) gewählt. Zur kinetischen Untersuchung bimolekularer Gasphasenreaktivitäten gegenüber einem neutralen Reaktionspartner erfolgte eine schrittweise Erhöhung der Fragmentierungszeit zwischen 0 und 6000 ms. Wurden Reaktionen in Lösung mittels ESI-Massenspektrometrie kinetisch betrachtet, erfolgte die Aufnahme von Massenspektren im Modus alternierender Polaritäten mit je 50 gemittelten $\mathrm{m} / \mathrm{z}$-Scans. In seltenen Fällen wurden hochaufgelöste Massenspektren mit einem micrO-TOF-Q IISpektrometer (Bruker Daltonik) aufgenommen, um die Zuordnung einzelner Peaks durch den Vergleich mit exakten Massen und simulierten Isotopenmustern zu verifizieren. Beide Massenspektrometer wurden extern mit einer kommerziell erworbenen Lösung aus Trifluoressigsäure und Phosphazenen in Wasser/Acetonitril kalibriert. Wurden die zuvor 
beschriebenen Standardparameter und -verfahren geändert, ist dies ausdrücklich bei der Darstellung der jeweiligen Ergebnisse erwähnt.

\subsubsection{Elektrische Konduktometrie}

Spezifische elektrische Leitfähigkeiten $\kappa$ wurden mit einem Seven Multi Instrument von Mettler Toledo mit einer Messelektrode aus Edelstahl (Kzelle $=0.1 \mathrm{~cm}^{-1}$ ) durchgeführt $(\rightarrow$ Abbildung 81). Die Kalibrierung der Messelektrode erfolgte gegen eine Lösung aus $\mathrm{KCl}$ in Wasser $(0.1 \mathrm{M})$ bei $298 \mathrm{~K}^{\left[{ }^{112]}\right.}$ Die Messzelle wurde unter Einsatz allgemeiner SchlenkTechniken vorbereitet und die Elektrode unter Argon-Gegenstrom eingesetzt. Nach Überführen der Probenlösung (Vorbereitung der Probe gemäß Kapitel 6.2.5) in die vorgekühlte Messzelle erfolgte die Messung der spezifischen, elektrischen Leitfähigkeit $\kappa$ bei $T=273 \mathrm{~K}$, um Hydrolyse- und Zerfallsreaktionen weitestgehend zu unterdrücken. lodometrische Titration der Grignard-Reagenzien (gemäß Kapitel 6.1.5) unmittelbar vor der Probenvorbereitung bestätigte deren Aktivität von $\geq 90 \%$ und ermöglichte eine sehr präzise Stöchiometrie der Probe.

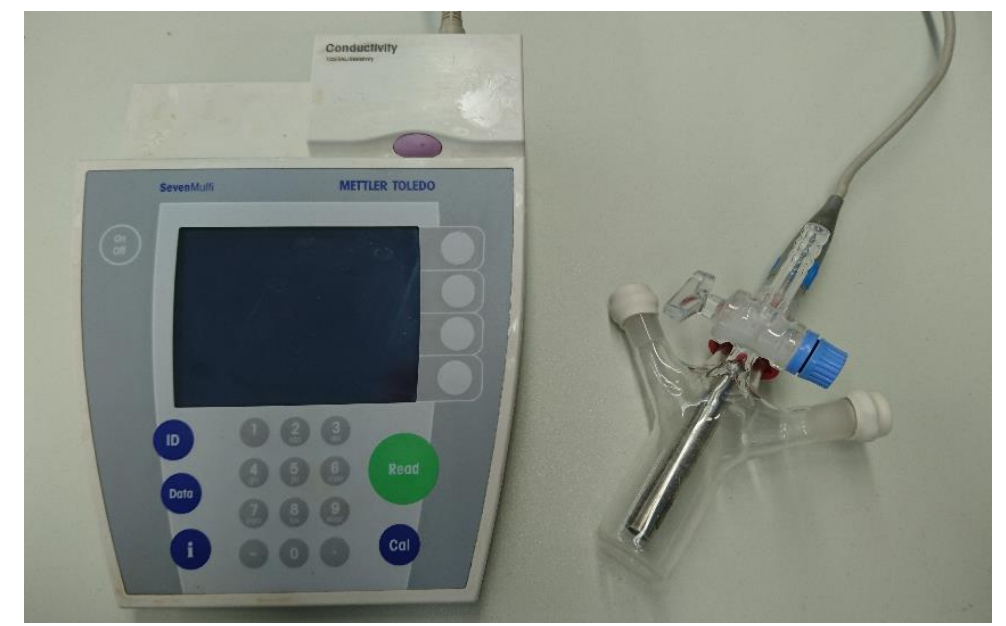

Abbildung 81. Seven Multi Instrument (Mettler Toledo) mit Edelstahlelektrode in Schlenk-fähiger Messzelle zur Messung der spezifischen elektrischen Leitfähigkeit $\kappa$ von hydrolyse- und oxidationsempfindlichen Probenlösungen.

\subsubsection{Mößbauer-Spektroskopie}

Mößbauer-Spektren wurden an einem Mößbauer-Spektrometer (Wissenschaftliche Elektronik $\mathrm{GmbH}$, WissEl) im Transmissionsmodus unter Verwendung einer ${ }^{57}$ Cobalt-Quelle in einer 
Rhodium-Matrix aufgenommen. ${ }^{v}$ Der Antrieb der Quelle erfolgte entsprechend einem Dreieckgeschwindigkeitsprofil mit konstanter Beschleunigung. Die Probe wurde mit einem Closed-Cycle-Kryostat (Janis) gekühlt. Die Isomerieverschiebungen $\delta$ sind gegen $\alpha$-Eisenfolie bei Umgebungstemperatur angegeben. Das Ansetzen der Probenlösungen (Probenvorbereitung gemäß Kapitel 6.2.5) und deren anschließender Transfer in die MößbauerMesszelle fanden unter Schutzgas-Atmosphäre in einer Glove-Box (M.Braun InertgasSysteme $\mathrm{GmbH}$ ) statt, um ungewollte Hydrolyse- und Oxidationsreaktionen zu vermeiden.

\footnotetext{
$\checkmark$ Mößbauer-Experimente, die im Rahmen dieser Arbeit präsentiert werden, fanden in Kooperation mit dem
} Arbeitskreis von Prof. Dr. F. Meyer in Person von Dr. S. Demeshko statt. 


\section{Literaturverzeichnis}

[1] Metal-Catalyzed Cross-Coupling Reactions and More, A. de Meijere, S. Bräse, M. Oestreich, Wiley VCH, Weinheim, 2014.

[2] (a) C. C. C. Johansson Seechurn, M. O. Kitching, T. J. Colacot, V. Snieckus, Angew. Chem. Int. Ed. 2012, 51, 5062-5085; Angew. Chem. 2012, 124, 5150-5174. (b) S. Xu, E. H. Kim, A. Wei, E.-I. Negishi, Sci. Technol. Adv. Mater. 2014, 15, 1-23.

[3] (a) C. Bolm, J. Legros, J. Le Paih, L. Zani, Chem Rev. 2004, 104, 6217-6254. (b) A. Fürstner, ACS Cent. Sci. 2016, 2, 778-789.

[4] C. Allègre, G. Manhès, E. Lewin, Earth \& Planetary Sci. Lett. 2001, 185, 49-69.

[5] (a) J. Legros, B. Figadère, Nat. Prod. Rep. 2015, 32, 1541-1555. (b) K. S. Egorova, V. P. Ananikov, Organometallics 2017, 36, 4071-4090.

[6] P. A. Frey, G. H. Reed, ACS Chem. Biol. 2012, 7, 1477-1481.

[7] (a) T. J. Lynch, M. Banah, H. D. Kaesz, C. R. Porter, J. Org. Chem. 1984, 49, 12661270. (b) F. Effenberger, D. Steegmüller, Chem. Ber. 1988, 121, 117-123. (c) T. Bach, D. N. A. Fox, M. T. Reetz, J. Chem. Soc., Chem. Commun. 1992, 1634-1636. (d) B. L. Small, M. Brookhart, A. M. Bennett, J. Am. Chem. Soc. 1998, 120, 4049-4050. (e) S. J. Mahmood, M. Hossain, J. Org. Chem. 1998, 63, 3333-3336. (f) D. F. Taber, K. Kanai, Q. Jiang, G. Bui, J. Am. Chem. Soc. 2000, 122, 6807-6808.

[8] W. M. Czaplik, M. Mayer, J. Cvengros, A. Jacobi von Wangelin, Chem. Sus. Chem. 2009, 2, 396-417.

[9] (a) N. Tewari, N. Maheshwari, R. Medhane, H. Nizar, M. Prasad, Org. Process Res. Dev. 2012, 16, 1566-1568. (b) S. M. Andersen, M. Bollmark, R. Berg, C. Fredriksson, S. Karlsson, C. Liljeholm, H. Sörensen, Org. Process Res. Dev. 2014, 18, 952-959. (c) S. Gangula, U. K. Neelam, S. R. Baddam, V. H. Dahanukar, R. Bandichhor, Org. Process Res. Dev. 2015, 19, 470-475. (d) D. Sengupta, T. Gharbaoui, A. Krishnan, D. J. Buzard, R. M. Jones, Y.-A. Ma, R. Burda, A. G. Montalban, G. Semple, Org. Process Res. Dev. 2015, 19, 618-623. (e) P. Mullens, E. Cleator, M. McLaughlin, B. Bishop, J. Edwards, A. Goodyear, T. Andrani, Y. Jin, J. Kong, H. Li, M. Williams, M. Zacuto, Org. Process Res. Dev. 2016, 20, 1075-1087. (f) T. Cernak, K. D. Dykstra, S. Tyagarajan, P. Vachal, S. W. Krska, Chem. Soc. Rev. 2016, 45, 546-576.

[10] (a) G. Cahiez, A. Moyeux, Chem. Rev. 2010, 110, 1435-1462. (b) B. M. Rosen, K. W. Quasdorf, D. A. Wilson, N. Zhang, A.-M. Resmerita, N. K. Garg, V. Percec, Chem. Rev. 2011, 111, 1346-1416. (c) S. Z. Tasker, E. A. Standley, T. F. Jamison, Nature 2014, 509, 299-310. (d) V. P. Ananikov, ACS Catal. 2015, 5, 1964-1971.

[11] (a) Palladium-Catalyzed Coupling Reactions: Practical Aspects and Future Developments, Á. Molnár, Wiley-VCH, Weinheim, 2013. (b) A. Piontek, E. Bisz, M. Szostak, Angew. Chem. Int. Ed. 2018, 57, 11116-11128; Angew. Chem. 2018, 130, 11284-11297.

[12] M. Kharasch, E. Fields, J. Am. Chem. Soc. 1941, 63, 2316-2320.

[13] M. Tamura, J. Kochi, Synthesis 1971, 6, 303-305.

[14] (a) K. G. Dongol, H. Koh, M. Sau, C. L. L. Chai, Adv. Synth. Catal. 2007, 349, 10151018. (b) M. Guisán-Ceinos, F. Tato, E. Buñuel, P. Calle, D. J. Cárdenas, Chem. Sci. 2013, 4, 1098-1104. 
[15] (a) A. Fürstner, A. Leitner, Angew. Chem. Int. Ed. 2002, 41, 609-612; Angew. Chem. 2002, 114, 632-635. (b) A. Fürstner, A. Leitner, M. Mendez, H. Krause, J. Am. Chem. Soc. 2002, 124, 13856-13863. (c) B. Barré, L. Gonnard, R. Champagne, S. Reymond, J. Marin, P. Ciapetti, M. Brellier, A. Guérinot, J. Cossy, Org. Lett. 2014, 16, 6160-6163. (d) R. Agata, T. Iwamoto, N. Nakagawa, K. Isozaki, T. Hatekayama, H. Takaya, M. Nakamura, Synthesis 2015, 47, 1733-1740. (e) T. Xing, Z. Zhang, Y.-X. Da, Z.-J. Quan, X.-C. Wang, Asian J. Org. Chem. 2015, 4, 538-544. (f) Z. Li, L. Liu, H.-M. Sun, Q. Shen, Y. Zhang, Dalton Trans. 2016, 45, 17739-17747.

[16] X. Chen, Z.-J. Quan, X.-C. Wang, Appl. Organomet. Chem. 2015, 29, 296-300.

[17] (a) M. Tamura, J. Kochi, J. Organomet. Chem. 1971, 31, 289-309. (b) S. M. Neumann, J. Kochi, J. Organomet. Chem. 1975, 40, 599-606. (c) G. Cahiez, H. Avedissian, Synthesis 1998, 8, 1199-1205. (d) C. M. Rao Volla, P. Vogel, Angew. Chem. Int. Ed. 2008, 47, 1305-1307; Angew. Chem. 2008, 120, 1325-1327. (e) B.-J. Li, Z.-H. Wu, B.-T. Guan, C.-L. Sun, B.-Q. Wang, Z.-J. Shi, J. Am. Chem. Soc. 2009, 131, 14656-14657. (f) D. Gärtner, A. L. Stein, S. Gruppe, J. Arp, A. Jacobi von Wangelin, Angew. Chem. Int. Ed. 2015, 54, 10545-10549; Angew. Chem. 2015, 127, 10691-10695.

[18] D. J. Tindall, H. Krause, A. Fürstner, Adv. Synth. Catal. 2016, 358, 2398-2403.

[19] (a) M. Nakamura, K. Matsuo, S. Ito, E. Nakamura, J. Am. Chem. Soc. 2004, 126, 36863687. (b) T. Nagano, T. Hayashi, Org. Lett. 2004, 6, 1297-1299. (c) R. B. Bedford, D. W. Bruce, R. M. Frost, J. W. Goodby, M. Hird, Chem. Commun. 2004, 2822-2823. (d) R. B. Bedford, D. W. Bruce, R. M. Frost, M. Hird, Chem. Commun. 2005, 4161-4163. (e) R. B. Bedford, M. Betham, D. W. Bruce, A. A. Danopoulos, R. M. Frost, M. Hird, J. Org. Chem. 2006, 71, 1104-1110. (f) R. B. Bedford, M. Betham, D. W. Bruce, S. A. Davis, R. M. Frost, M. Hird, Chem. Commun. 2006, 1398-1400. (g) W. Czaplik, M. Mayer, A. Jacobi von Wangelin, Angew. Chem. Int. Ed. 2009, 48, 607-610; Angew. Chem. 2009, 121, 616620. (h) W. Czaplik, M. Mayer, S. Gruppe, A. Jacobi von Wangelin, Pure Appl. Chem. 2010, 82, 1545-1553. (i) K. Weber, E.-M. Schnöckelborg, R. Wolf, Chem. Cat. Chem. 2011, 3, 1572-1577. (j) C.-L. Sun, H. Krause, A. Fürstner, Adv. Synth. Catal. 2014, 356, 1281-1291.

[20] (a) A. Molander, B. J. Rahn, D. C. Shubert, Tetrahedron Lett. 1983, 24, 5449-5452. (b) W. Dohle, F. Kopp, G. Cahiez, P. Knochel, Synlett. 2001, 12, 1901-1904. (c) G. Cahiez, V. Habiak, C. Duplais, A. Moyeux, Angew. Chem. Int. Ed. 2007, 46, 4364-4366; Angew. Chem. 2007, 119, 4442-4444. (d) W. Czaplik, M. Mayer, A. Jacobi von Wangelin, Chem. Cat. Chem. 2011, 3, 135-138. (e) R. N. Shakhmaev, A. S. Sunagatullina, V. V. Zorin, Russ. J. Org. Chem. 2014, 50, 322-331. (f) T. Agrawal, S. P. Cook, Org. Lett. 2014, 16, 5080-5083. (g) T. Iwasaki, R. Akimoto, H. Kuniyasu, N. Kambe, Chem. Asian J. 2016, $11,2834-2837$.

[21] (a) T. Hatakeyama, M. Nakamura, J. Am. Chem. Soc. 2007, 129, 9844-9845. (b) T. Hatakeyama, S. Hashimoto, K. Ishizuka, M. Nakamura, J. Am. Chem. Soc. 2009, 131, 11949-11963. (c) Y.-Y. Chua, H. A. Duong, Chem. Commun. 2014, 50, 8424-8427. (d) W. Wu, Q. Teng, Y.-Y. Chua, H. V. Huynh, H. A. Duong, Organometallics, 2017, 36, 2293-2297.

[22] (a) J. Quintin, X. Franck, R. Hooquemiller, B. Figadere, Tetrahedron Lett. 2002, 43, 35473549. (b) O. M. Kuzmina, A. K. Steib, J. T. Markiewicz, D. Flubacher, P. Knochel, Angew. Chem. Int. Ed. 2013, 52, 4945-4949; Angew. Chem. 2013, 125, 5045-5049.

[23] A. Guérinot, S. Reymond, J. Cossy, Angew. Chem. Int. Ed. 2007, 46, 6521-6524; Angew. Chem. 2007, 119, 6641-6644. 
[24] C. W. Cheung, P. Ren, X. Hu, Org. Lett. 2014, 16, 2566-2569.

[25] T. Hatekayama, Y. Yoshimoto, T. Gabriel, M. Nakamura, Org. Lett. 2008, 10, 5341-5344.

[26] (a) G. Bauer, C. W. Cheung, X. Hu, Synthesis 2015, 47, 1726-1732. (b) M. Jin, L. Adak, M. Nakamura, J. Am. Chem. Soc. 2015, 137, 7128-7134.

[27] (a) M. Nakamura, S. Ito, K. Matsuo, E. Nakamura, Synlett. 2005, 11, 1794-1798. (b) R. B. Bedford, M. Huwe, M. C. Wilkinson, Chem. Commun. 2009, 600-602. (c) S. Ito, Y.-I. Fujiwara, E. Nakamura, M. Nakamura, Org. Lett. 2009, 11, 4306-4309. (d) G. Cahiez, L. Foulgoc, A. Moyeux, Angew. Chem. Int. Ed. 2009, 48, 2969-2972; Angew. Chem. 2009, 121, 3013-3016. (e) R. B. Bedford, E. Carter, P. M. Cogswell, N. J. Gower, M. F. Haddow, J. N. Harvey, D. M. Murphy, E. C. Neeve, J. Nunn, Angew. Chem. Int. Ed. 2013, 52, 1285-1288; Angew. Chem. 2013, 125, 1323-1326. (f) K. J. Gallagher, R. L. Webster, Chem. Commun. 2014, 50, 12109-12111. (g) R. B. Bedford, P. B. Brenner, E. Carter, J. Clifton, P. M. Cogswell, N. J. Gower, M. F. Haddow, J. N. Harvey, J. A. Kehl, D. M. Murphy, E. C. Neeve, M. L. Neidig, J. Nunn, B. E. R. Snyder, J. Taylor, Organometallics 2014, 33, 5767-5780. (h) J. Clifton, E. R. M. Habraken, P. G. Pringle, I. Manners, Catal. Sci. Technol. 2015, 5, 4350-4353. (i) C. A. Brown, T. A. Nile, M. F. Mahon, R. L. Webster, Dalton Trans. 2015, 44, 12189-12195. (j) A. D. Benischke, M. Leroux, I. Knoll, P. Knochel, Org. Lett. 2016, 18, 3626-3629.

[28] (a) T. Hatekayama, T. Hashimoto, Y. Kondo, Y. Fujiwara, H. Seike, H. Takaya, Y. Tamada, T. Ono, M. Nakamura, J. Am. Chem. Soc. 2010, 132, 10674-10676. (b) R. B. Bedfrod, P. B. Brenner, E. Carter, T. W. Carvell, P. M. Cogswell, T. Gallagher, J. N. Harvey, D. M. Murphy, E. C. Neeve, J. Nunn, D. R. Pye, Chem. Eur. J. 2014, 20, 79357938. (c) T. Atack, R. M. Lecker, S. P. Cook, J. Am. Chem. Soc. 2014, 136, 9521-9523. (d) R. B. Bedford, T. Gallagher, D. R. Pye, W. Savage, Synthesis 2015, 47, 1761-1765. (e) J. Dunsford, E. R. Clark, M. J. Ingleson, Dalton Trans. 2015, 44, 20577-20583. (f) T. Yoshida, L. Ilies, E. Nakamura, ACS Catalysis 2017, 7, 3199-3203.

[29] S. Kawarnura, R. Agata, M. Nakamura, Org. Chem. Frontiers 2015, 2, 1053-1058.

[30] (a) H. M. Walborsky, R. B. Banks, J. Org. Chem. 1981, 46, 5074-5077. (b) Z. Jia, X.-S. Peng, H. N. C. Wong, Nat. Comm. 2016, 7, 10614-10620.

[31] (a) G. Cahiez, S. Marquais, Pure \& Appl. Chem. 1996, 68, 53-60. (b) A. D. Benischke, A. J. A. Breuillac, A. Moyeux, G. Cahiez, P. Knochel, Synlett. 2016, 27, 471-476.

[32] W.-J. Shi, H.-W. Zhao, Y. Wang, Z.-C. Cao, L.-S. Zhang, D.-G. Yu, Z.-J. Shi, Adv. Synth. Catal. 2016, 358, 2410-2416.

[33] (a) I. Sapountzis, W. Lin, C. C. Kofink, C. Despotopoulou, P. Knochel, Angew. Chem. Int. Ed. 2005, 44, 1654-1658; Angew. Chem. 2005, 117, 1682-1685. (b) C. C: Kofink, B. Blank, S. Pagano, N. Goetz, P. Knochel, Chem. Commun. 2007, 1954-1956.

[34] K. M. Liu, J. Wie, X. F. Duan, Chem. Commun. 2015, 51, 4655-4658.

[35] (a) J. K. Kochi, J. Organomet. Chem. 2002, 653, 11-19. (b) A. Fürstner, R. Martin, H. Krause, G. Seidel, R. Goddard, C. W. Lehmann, J. Am. Chem. Soc. 2008, 130, 87738787. (c) J. Kleimark, A. Hedström, P.-F. Larsson, C. Johansson, P.-O. Norrby, Chem. Cat. Chem. 2009, 1, 152-161. (d) A. Hedström, E. Lindstedt, P.-O- Norrby, J. Organomet. Chem. 2013, 748, 51-55. (e) R. Schoch, W. Desens, T. Werner, M. Bauer, Chem. Eur. J. 2013, 19, 15816-15821. (f) G. Lefèvre, A. Jutand, Chem. Eur. J. 2014, 20, 4796-4805. (g) A. Bekhradnia, P.-O. Norrby, Dalton Trans. 2015, 44, 3959-3962. (h) A. Hedström, Z. Izakian, I. Vreto, C.-J. Wallentin, P.-O. Norrby, Chem. Eur. J. 2015, 21, 5946-5953. 
[36] (a) D. Noda, Y. Sunada, T. Hatekayama, M. Nakamura, H. Nagashima, J. Am. Chem. Soc. 2009, 131, 6078-6079. (b) J. A. Przyojski, K. P. Veggeberg, H. D. Arman, Z. J. Tonzetich, ACS Catalysis 2015, 5, 5938-5946. (c) G. Bauer, M. D. Wodrich, R. Scopelliti, X. Hu, Organometallics 2015, 34, 289-298. (d) Y. Liu, J. Xiao, L. Wang, Y. Song, L. Deng, Organometallics 2015, 34, 599-605.

[37] (a) B. Bogdanovic, M. Schwickardi, Angew. Chem. Int. Ed. 2000, 39, 4610-4612; Angew. Chem. 2000, 112, 4788-4790. (b) A. Fürstner, A. Leitner, Angew. Chem. Int. Ed. 2002, 41, 609-612; Angew. Chem. 2002, 114, 632-635. (c) A. Fürstner, A. Leitner, M. Mendez, H. Krause, J. Am. Chem. Soc. 2002, 124, 13856-13863.

[38] T. Parchomyk, K. Koszinowski, Synthesis 2017, 49, 3269-3280.

[39] (a) K. Jonas, L. Schieferstein, C. Krüger, Y.-H. Tsay, Angew. Chem Int. Ed. 1979, 18, 550-551; Angew. Chem. 1979, 91, 550-551. (b) T. A. Bazhenova, L. M. Kachapina, A. E. Shilov, J. Organomet. Chem. 1992, 428, 107-123. (c) P. J. Alonso, A. B. Arauzo, J. Fornies, M. A. Garcia-Monforte, A. Martin, J. I. Martinez, B. Menjón, C. Rillo, J. J. SáizGaritaonandia, Angew. Chem. Int. Ed. 2006, 45, 6707-6711; Angew. Chem. 2006, 118, 6859-6863. (d) C.-L. Sun, H Krause, A. Fürstner, Adv. Synth. Catal. 2014, 356, 12811291. (e) R. Bedford, P. B. Brenner, E. Carter, P. M. Cogswell, M. F. Haddow, J. N. Harvey, D. M. Murphy, J. Nunn, C. H. Woodall, Angew. Chem. Int. Ed. 2014, 53, 18041808; Angew. Chem. 2014, 126, 1835-1839. (f) M. H. Al-Afyouni, K. L. Fillman, W. W. Brennessel, M. L. Neidig, J. Am. Chem. Soc. 2014, 136, 15457-15460. (g) S. B. Muñoz III, S. L. Daifuku, W. W. Brennessel, M. L. Neidig, J. Am. Chem. Soc. 2016, 138, 74927495. (h) F. E. Zhurkin, M. D. Wodrich, X. Hu, Organometallics 2017, 36, 499-501. (i) S. B. Muñoz III, S. L. Daifuku, J. D. Sears, T. M. Baker, S. H. Carpenter, W. W. Brennessel, M. L. Neidig, Angew. Chem. Int. Ed. 2018, 57, 6496-6500; Angew. Chem. 2018, 130, 6606-6610.

[40] D. A. Plattner, Int. J. Mass. Spectrom. 2001, 207, 125-144.

[41] (a) S. L. Daifuku, J. L. Kneebone, B. E. R. Snyder, M. L. Neidig, J. Am. Chem. Soc. 2015, 137, 11432-11444. (b) J. L. Kneebone, V. E. Fleischauer, S. L. Daifuku, A. A. Shaps, J. M. Bailey, T. E. lannuzzi, M. L. Neidig, Inorg. Chem. 2016, 55, 272-282. (c) J. L. Kneebone, W. W. Brennessel, M. L. Neidig, J. Am. Chem. Soc. 2017, 139, 6988-7003.

[42] Massenspektrometrie - Ein Lehrbuch, J. H. Gross, Springer-Verlag, Berlin, 2013.

[43] H. Wollnik, J. Mass. Spectrom. 1999, 34, 991-1006.

[44] M. Dole, L. L. Mack, R. L. Hines, R. C. Mobley, L. D. Ferguson, M. B. Alice, J. Chem. Phys. 1968, 49, 2240-2249.

[45] M. Yamashita, J. B. Fenn, J. Phys. Chem. 1984, 88, 4451-4459.

[46] N. B. Cech, C. G. Enke, Mass. Spectrom. Rev. 2001, 20, 362-387.

[47] (a) P. Kebarle, U. H. Verkerk, Mass. Spectrom. Rev. 2009, 28, 898-917. (b) L. Konermann, E. Ahadi, A. D. Rodriguez, S. Vahidi, Anal. Chem. 2013, 85, 2-9.

[48] J. V. Iribarne, B. A. Thomson, J. Chem. Phys. 1976, 64, 2287-2294.

[49] T. C. Rohner, N. Lion, H. H. Girault, Phys. Chem. Chem. Phys. 2004, 6, 3056-3068.

[50] (a) A. R. Pitt, Nat. Prod. Rep. 1998, 15, 59-72. (b) S. Mehmood, T. M. Allison, C. V. Robinson, Annu. Rev. Phys. Chem. 2015, 66, 453-474.

[51] A. Marchand, V. Gabelica, J. Am. Soc. Mass. Spectrom. 2014, 25, 1146-1154. 
[52] (a) M. Buback, H. Frauendorf, F. Günzler, F. Huff, P. Vana, Macromol. Chem. Phys. 2009, 210, 1591-1599. (b) N. F. König, A. Al Ouahabi, S. Poyer, L. Charles, J.-F. Lutz, Angew. Chem. Int. Ed. 2017, 56, 7297-7301; Angew. Chem. 2017, 129, 7403-7407.

[53] (a) M. Herderich, E. Richling, R. Roscher, C. Schneider, W. Schwab, H.-U. Humpf, P. Schreier, Chromatographia 1997, 45, 127-132. (b) M. A. Strege, J. Chromatogr. B. Biomed. Sci. Appl. 1999, 725, 67-78.

[54] (a) R. J. Oeschger, D. H. Ringger, P. Chen, Organometallics 2015, 34, 3888-3892. (b) K. L. Vikse, P. Chen, Organometallics 2015, 34, 1294-1300. (c) J. Penafiel, A. V. Hesketh, O. Granot, J. S. Mclndoe, Dalton Trans. 2016, 45, 15552-15556. (d) J. Li, J. M. White, R. J. Mulder, G. E. Reid, P. S. Donnelly, R. A. J. O'Hair, Inorg. Chem. 2016, 55, 9858-9868. (e) E. Janusson, H. S. Zijlstra, P. T. Nguyen, L. MacGillivray, J. Martelino, J. S. Mclndoe, Chem. Commun. 2017, 53, 854-856. (f) J. Váña, T. Terencio, V. Petrovic, O. Tischler, Z. Novák, J. Roithová, Organometallics 2017, 36, 2072-2080. (g) G. Yassaghi, E. Andris, J. Roithová, Chem. Phys. Chem. 2017, 18, 2217-2224. (h) M. Lesslie, Y. Yang, A. J. Canty, E. Piacentino, F. Berthias, P. Maitre, V. Ryzhov, R. A. J. O'Hair, Chem. Commun. 2018, 54, 346-349.

[55] (a) K. Koszinowski, P. Böhrer, Organometallics 2009, 28, 100-110. (b) K. Koszinowski, J. Am. Chem. Soc. 2010, 132, 6032-6040. (c) A. Putau, H. Brand, K. Koszinowski, J. Am. Chem. Soc. 2012, 134, 613-622. (d) C. Schnegelsberg, S. Bachmann, M. Kolter, T. Auth, M. John, D. Stalke, K. Koszinowski, Chem. Eur. J. 2016, 22, 7752-7762. (e) M. Kolter, K. Koszinowski, Chem. Eur. J. 2016, 22, 15744-15750. (f) F. Kreyenschmidt, K. Koszinowski, Chem. Eur. J. 2018, 24, 1168-1177. (g) S. Weske, R. A. Hardin, T. Auth, R. A. J. O`Hair, K. Koszinowski, C. A. Ogle, Chem. Commun. 2018, 54, 5086-5089.

[56] Mass Spectrometry of Inorganic, Coordination and Organometallic Compounds, W. Henderson, J. S. Mclndoe, John Wiley \& Sons Ltd., Chichester, 2005.

[57] G. J. Van Berkel, K. G. Asano, P. D. Schnier, J. Am. Soc. Mass. Spectrom. 2001, 12, 853-862.

[58] R. A. J. O`Hair, N. J. Rijs, Acc. Chem. Res. 2015, 46, 329-340.

[59] D. W. Koppenaal, G. C. Eiden, C. J. Barinaga, J. Anal. At. Spectrom. 2004, 19, 561-570.

[60] (a) S. Gronert, Chem. Rev. 2001, 101, 329-360. (b) R. A. J. O`Hair, Chem. Comm. 2006, 1469-1481.

[61] S. Gronert, Mass. Spectrom. Rev. 2005, 24, 100-120.

[62] Lehrbuch der Physikalischen Chemie, G. Wedler, Wiley-VCH, Weinheim, 2004.

[63] Physikalische Chemie, P. W. Atkins, J. de Paula, Wiley-VCH, Weinheim, 2006.

[64] (a) A. Putau, K. Koszinowski, Organometallics 2011, 30, 4771-4778. (b) J. E. Fleckenstein, K. Koszinowski, Organometallics 2011, 30, 5018-5026. (c) A. Putau, H. Brand, K. Koszinowski, J. Am. Chem. Soc. 2012, 134, 613-622. (d) A. Putau, M. Wilken, K. Koszinowski, Chem. Eur. J. 2013, 19, 10992-10999. (e) M. Kolter, K. Böck, K. Karaghiosoff, K. Koszinowski, Angew. Chem. Int. Ed. 2017, 56, 13244-13248; Angew. Chem. 2017, 129, $13427-13431$.

[65] R. L. Mößbauer, Hyperfine Interactions 2000, 126, 1-12.

[66] The Nobel Prize in Physics 1961. Nobelprize.org, Nobel Media AB 2014, Web, Zugriff am 18 Mai 2018, <http://www.nobelprize.org/nobel_prizes/physics/laureates/1961/>.

[67] P. Gütlich, H. Prange, Chemie-Ing.-Techn. 1971, 19, 1049-1061. 
[68] (a) H. Auerbach, G. E. Giammanco, V. Schünemann, A D. Ostrowski, C. J. Carrano, Inorg. Chem. 2017, 56, 11524-11531. (b) J. L. Kneebone, S. L. Daifuku, J. A. Kehl, G. Wu, H. T. Chung, M. Y. Hu, E. E. Alp, K. L. More, P. Zelenay, E. F. Holby, M. L. Neidig, J. Phys. Chem. C 2017, 121, 16283-16290. (c). J. Tong, S. Demeshko, S. Dechert, F. Meyer, Eur. J. Inorg. Chem. 2017, 37, 4333-4343. (d) L. Wang, M. Zlatar, F. Vlahovic, S. Demeshko, C. Philouze, F. Molton, M. Gennari, F. Meyer, C. Duboc, M. Gruden, Chem. Eur. J. 2018, 24, 5091-5094.

[69] (a) A. de Kergommeaux, J. Faure-Vincent, A. Pron, R. de Bettignies, B. Malaman, P. Reiss, J. Am. Chem. Soc. 2012, 134, 11659-11666. (b) K. E. Johnston, M. T. Sougrati, L. Stievano, A. Darwiche, N. Dupré, C. P. Grey, L. Monconduit, Chem. Mater. 2016, 28, 4032-4041. (c) G. A. Sundaram, M. Yang, K. Nomura, S. Maniarasu, G. Veerappan, T. Liu, J. Wang, J. Phys. Chem. C 2017, 121, 6662-6673.

[70] (a) E. Bychkov, G. Wortmann, J. Non-Cry. Sol. 1993, 159, 162-172. (b) M. Radzieowski, T. Block, T. Fickenscher, Y. Zhang, B. P. T. Fokwa, O. Janka, Mater. Chem. Front. 2017, 1, 1563-1572. (c) C. Benndorf, L. Heletta, T. Block, H. Eckert, R. Pöttgen, Z. Anorg. Allg. Chem. 2017, 643, 294-298.

[71] (a) H. Kitagawa, N. Onodera, T. Sonoyama, M. Yamamoto, T. Fukawa, T. Mitani, M. Seto, Y. Maeda, J. Am. Chem. Soc. 1999, 121, 10068-10080. (b) A. Kobayashi, S. Kitao, M. Seto, R. Ikeda, H. Kitagawa, Inorg. Chem. 2009, 48, 8044-8049. (c) T. Zelovich, A. Borschevsky, E. Eliav, U. Kaldor, Mol. Phys. 2017, 115, 138-143.

[72] P. Gütlich, Z. Anorg. Allg. Chem. 2012, 638, 15-43.

[73] Lexikon der Chemie, H.-D. Jakubke, R. Karcher, Spektrum Akademischer Verlag GmbH, Heidelberg, 2008.

[74] G. Winter, D. W. Thompson, J. R. Loehe, Inorg. Synth. 1973, 14, 99-104.

[75] C. Schnegelsberg, T. D. Blümke, K. Koszinowski, J. Mass. Spectrom. 2015, 50, 13931395.

[76] (a) V. W. Seidel, K. J. Lattermann, Z. Anorg. Allg. Chem. 1982, 488, 69-74. (b) A. Klose, E. Solari, C. Floriani, A. Chiesi-Villa, C. Rizzoli, N. Re, J. Am. Chem. Soc. 1994, 116, 9123-9135. (c) E. J. Hawrelak, W. H. Bernskoetter, E. Lobkovsky, G. T. Yee, E. Bill, P. J. Chirik, Inorg. Chem. 2005, 44, 3103-3111. (d) M. Irwin, R. K. Jenkins, M. S. Denning, T. Krämer, F. Grandjean, G. J. Long, R. Herchel, J. E. McCrady, J. M. Goicoechea, Inorg. Chem. 2010, 49, 6160-6171. (e) S. L. Daifuku, M. H. Al-Afyouni, B. E. R. Snyder, J. L. Kneebone, M. L. Neidig, J. Am. Chem. Soc. 2014, 136, 9132-9143.

[77] M. Clémancey, T. Cantat, G. Blondin, J.-M. Latour, P. Dorlet, G. Lefèvre, Inorg. Chem. 2017, 56, 3834-3848.

[78] Anorganische Chemie, E. Riedel, Walter de Gruyter GmbH \& Co.KG, Berlin, 2004.

[79] Organic Chemistry, J. Clayden, N. Greeves,S. Warren, Oxford University Press, New York, 2012.

[80] A. Casitas, J. A. Rees, R. Goddard, E. Bill, S. DeBeer, A. Fürstner, Angew. Chem. Int. Ed. 2017, 56, 10108-10113; Angew. Chem. 2017, 129, 10242-10247.

[81] (a) B. M. Prince, T. R. Cundari, C. J. Tymczak, J. Phys. Chem. A 2014, 118, 1105611061. (b) J. Mei, D. B. Pardue, S. E. Kalman, T. B. Gunnoe, T. R. Cundari, M. Sabat, Organometallics 2014, 33, 5597-5605.

[82] R. B. Bedford, P. B. Brenner, D. Elorriaga, J. N. Harvey, J. Nunn, Dalton Trans. 2016, $45,15811-15817$. 
[83] (a) A. G. Orpen, N. G. Connelly, Organometallics 1990, 9, 1206-1210. (b) The Organometallic Chemistry of the Transition Metals, R. H. Crabtree, Wiley-VCH, Weinheim, 2014.

[84] T. A. Albright, R. Hoffmann, J. C. Thibeault, D. L. Thorn, J. Am. Chem. Soc. 1979, 101, 3801-3812.

[85] (a) M. J. S. Dewar, Bull. Soc. Chim. Fr. 1951, 18, C71. (b) J. Chatt, L. A. Duncanson, J. Chem. Soc. 1953, 2939-2947.

[86] (a) J. A. Przyojski, H. D. Arman, Z. J. Tonzetich, Organometallics 2012, 31, 3264-3271. (b) K. L. Fillman, J. A. Przyojski, M. H. Al-Afyouni, Z. J. Tonzetich, M. L. Neidig, Chem. Sci. 2015, 6, 1176-1188.

[87] (a) C. Hinderling, C. Adlhart, P. Chen, Angew. Chem. Int. Ed. 1998, 37, 2685-2689; Angew. Chem. 1998, 110, 2831-2835. (b) C. Adlhart, C. Hinderling, H. Baumann, P. Chen, J. Am. Chem. Soc. 2000, 122, 8204-8214. (c) D. M. Chisholm, J. S. Mclndoe, Dalton Trans. 2008, 3933-3945. (d) E. Crawford, T. Lohr, E. M. Leitao, S. Kwok, J. S. Mclndoe, Dalton Trans. 2009, 9110-9112. (e) M. A. Schade, J. E. Fleckenstein, P. Knochel, K. Koszinowski, J. Org. Chem. 2010, 75, 6848-6857.

[88] A. Casitas, J. A. Rees, R. Goddard, E. Bill, S. DeBeer, A. Fürstner, Angew. Chem. Int. Ed. 2017, 56, 10108-10113; Angew. Chem. 2017, 129, 10242-10247.

[89] R. B. Bedford, M. Betham, D. W. Bruce, S. A. Davis, R. M. Frost, M. Hird, Chem. Commun. 2006, 1398-1400.

[90] (a) W. N. Olmstead, J. I. Brauman, J. Am. Chem. Soc. 1977, 99, 4219-4928. (b) S. Gronert, C. H. DePuy, V. Bierbaum, J. Am. Chem. Soc. 1991, 113, 4010-4013.

[91] T. Waters, R. A. J. O`Hair, A. G. Wedd, J. Am. Chem. Soc. 2003, 125, 3384-3396.

[92] Comprehensive Handbook of Chemical Bond Energies, Y. R. Luo, CRC Press, Boca Raton, 2007.

[93] (a) T. Su, J. Chem. Phys. 1988, 88, 4102-4103; Korrektur: J. Chem. Phys. 1988, 89, 5355. (b) K. Kimura, S. Nagakura, Spectrochimica Acta 1961, 17, 166-183. (c) CRC Handbook of Chemistry and Physics, W. M. Haynes, CRC Press, Boca Raton, 2014.

[94] Organic Chemistry: Structure, Mechanism, Synthesis, J. D. Rawn, R. J. Ouellette, Elsevier, London, 2018.

[95] C. Hansch, A. Leo, W. Taft, Chem. Rev. 1991, 91, 165-195.

[96] S. Weske, R. Schoop, K. Koszinowski, Chem. Eur. J. 2016, 22, 11310-11316.

[97] R. F. Gunion, M. K. Gilles, M. L. Polak, W. C. Lineberger, Int. J. Mass. Spectrom. Ion Processes 1992, 117, 601-620.

[98] (a) K. Tatsumi, R. Hoffmann, A. Yamamoto, J. K. Stille, Bull. Chem. Soc. Jpn. 1981, 54, 1857-1867. (b) J. J. Low, W. A. Goddard III, J. Am. Chem. Soc. 1986, 108, 6115-6128.

[99] S. Shekhar, J. F. Hartwig, J. Am. Chem. Soc. 2004, 126, 13016-13027.

[100] (a) M. S. Driver, J. F. Hartwig, J. Am. Chem. Soc. 1997, 119, 8232-8245. (b) P. L. Arrechea, S. L. Buchwald, J. Am. Chem. Soc. 2016, 138, 12486-12493.

[101] K. Muñiz, Angew. Chem. Int. Ed. 2009, 48, 9412-9423; Angew. Chem. 2009, 121, 95769588. (b) K. M. Engle, T.-S. Mei, X. Wang, J.-Q. Yu, Angew. Chem. Int. Ed. 2011, 50, 1478-1491; Angew. Chem. 2011, 123, 1514-1528. 
[102] R. Liyanage, X.-G. Zhang, P. B. Armentrout, J. Chem. Phys. 2001, 115, 9747-9763.

[103] J. L. Loup, D. Zell, J. C. A. Oliveira, H. Keil, D. Stalke, L. Ackermann, Angew. Chem. Int. Ed. 2017, 56, 14197-14201; Angew. Chem. 2017, 129, 14385-14389.

[104] W. Schlenk, A. Thal, Ber. Dtsch. Chem. Ges. 1913, 46, 2840-2854.

[105] Maßanalyse, G. Jander, K.-F. Jahr, Walter de Gruyter GmbH \& Co.KG, Berlin, 2009.

[106] A. Krasovskiy, P. Knochel, Synthesis 2006, 5, 890-891.

[107] M. F. Lipton, C. M. Sorensen, A. C. Sadler, J. Organomet. Chem. 1980, 186, 155-158.

[108] M. Rottländer, L. Boymond, L. Bérillon, A. Leprêtre, G. Varchi, S. Avolio, H. Laaziri, G. Quéguiner, A. Ricci, G. Cahiez, P. Knochel, Chem. Eur. J. 2000, 6, 767-770.

[109] D. L. Comins, D. H. LaMunyon, Tetrahedron Lett. 1988, 29, 773-776.

[110] J. D. White, M. S. Jensen, Tetrahedron 1995, 51, 5743-5756.

[111] D. Rasina, M. Otikovs, J. Leitans, R. Recacha, V. Borysov, I. Kanepe-Lapsa, I. Domraceva, T. Pantelejevs, K. Tars, M. J. Blackman, K. Jaudzems, A. Jirgensons, J. Med. Chem. 2016, 59, 374-387.

[112] Y. C. Wu, W. F. Koch, J. Sol. Chem. 1991, 20, 391-401. 